\title{
Synthesis and characterization of smart block copolymers for biomineralization and biomedical applications
}

by

\section{Mathumai Kanapathipillai}

\author{
A dissertation submitted to the graduate faculty \\ in partial fulfillment of the requirements for the degree of \\ DOCTOR OF PHILOSOPHY
}

\author{
Major: Chemical and Biological Engineering \\ Program of Study Committee: \\ Surya K. Mallapragada, Major Professor \\ Mufit Akinc \\ Klaus Schmidt-Rohr \\ Balaji Narasimhan \\ Monica H. Lamm
}

Iowa State University

Ames, Iowa

2008

Copyright (c) Mathumai Kanapathipillai, 2008. All rights reserved. 


\section{TABLE OF CONTENTS}

$\begin{array}{cll}\text { CHAPTER } 1 & \text { INTRODUCTION } & 1 \\ \text { 1.1. } & \text { Introduction } \\ \text { 1.2. } & \text { Thesis organization } & 2 \\ 1.3 . & \text { References }\end{array}$

CHAPTER 2 BACKGROUND AND LITERATURE REVIEW 5

2.1. Smart block copolymers 5

2.2. Self-assembly-micellization and gelation of block copolymers 6

2.2.1. Theoretical aspects 8

2.2.2. Examples of micellar systems 9

2.3. Stimuli-responsive block copolymers 14

2.3.1. $\quad \mathrm{pH}$ responsive block copolymers 14

2.3.2. Temperature responsive block copolymers 17

2.3.3. Glucose responsive block copolymers 20

2.3.4. Other stimuli responsive and multi responsive

2.4. Hydrogels $\quad 22$

2.4.1. Stimuli responsive hydrogels 22

2.4.2. Artificial polypeptide hydrogels 26

2.5. Polymer synthetic methods 28

2.5.1. Living radical polymerization 28

2.5.2. Atom transfer radical polymerization 29

2.5.3. Reversible addition fragmentation chain transfer 30

2.5.4. N-carboxy anhydride polymerization 31

2.6. Characterization of smart copolymer supramolecular self-assembly 32

2.6.1. Spectroscopic techniques 32

2.6.2. Separation techniques 32

2.6.3. Scattering techniques 33

2.6.3.1. Laser light scattering 33

2.6.3.2. Small angle x-ray and neutron scattering 34

2.6.4. Cryogenic transmission electron microscopy 36

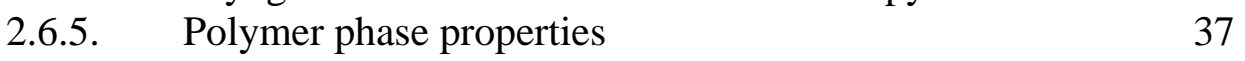

2.6.6. Mechanical properties 38

2.7. Applications of block copolymers in biomineralization 39

2.7.1. Naturally occurring hierarchical structures 39

2.7.2. Natural mineral formation process-bone as an 40

2.7.3. Biomimetic mineralization for hierarchically $\quad 42$

2.7.4. Hierarchical organization of block copolymers 43

2.7.5. Biomimetic mineralization using polymer templates $\quad 48$

2.7.5.1. Block copolymers and other polymeric materials in Biomineralization 
2.7.5.2. Biopolymers in biomineralization 52

2.8. Biomedical applications of stimuli responsive polymers 54

2.8.1. Drug depot 54

2.8.2. Injectable photocrosslinkable hydrogels for posttraumatic arthritis cartilage tissue healing $\quad 57$

2.8.3. Non-viral vectors for gene delivery $\quad 60$

2.9 References 63

$\begin{array}{lll}\text { CHAPTER } 3 & \text { RESEARCH OBJECTIVES }\end{array}$

3.1. Introduction 77

3.2. Specific goals of research 77

3.2.1. Develop block copolymer templates for $\quad 77$

3.2.2 Applications of these polymers in biomedical field 78

$\begin{array}{lll}\text { CHAPTER } 4 & \text { SYNTHESIS AND CHARACTERIZATION OF }\end{array}$

$\begin{array}{lll}4.1 & \text { Abstract } & 79\end{array}$

$\begin{array}{lll}4.2 & \text { Introduction } & 80\end{array}$

$\begin{array}{lll}4.3 & \text { Materials and methods } & 84\end{array}$

$4.4 \quad$ Characterization of polymer-gel nanocomposites 86

$\begin{array}{lll}4.4 & \text { Results and discussion } & 90\end{array}$

$\begin{array}{lll}4.5 & \text { Conclusions } & 97\end{array}$

$\begin{array}{ll}4.6 & \text { Acknowledgements }\end{array}$

$\begin{array}{lll}4.7 & \text { References } & 98\end{array}$

CHAPTER 5 SYNTHESIS AND CHARACTERIZATION OF IONIC BLOCK COPOLYMER TEMPLATED CALCIUM

PHOSPHATE NANOCOMPOSITES

$\begin{array}{lll}\text { 5.1. } & \text { Abstract } & 100\end{array}$

$\begin{array}{lll}\text { 5.2. Introduction } & 101\end{array}$

$\begin{array}{ll}\text { 5.3. Materials and methods } & 104\end{array}$

$\begin{array}{lll}\text { 5.4. } & \text { Results and discussions } & 109\end{array}$

$\begin{array}{lll}\text { 5.5. Conclusions } & 120\end{array}$

$\begin{array}{ll}\text { 5.6. Acknowledgements } & 121\end{array}$

$\begin{array}{lll}\text { 5.7. References } & 121\end{array}$

CHAPTER 6 BIOINSPIRED SYNTHESIS OF SELF-ASSEMBLED

CALCIUM PHOSPHATE NANOCOMPOSITES USING BLOCK COPOLYMER PEPTIDE CONJUGATES 123

$\begin{array}{lll}\text { 6.1. Abstract } & 123\end{array}$

$\begin{array}{lll}\text { 6.2. Introduction } & 124\end{array}$

$\begin{array}{ll}\text { 6.3. } & 125\end{array}$ 
6.4. Results and discussion 132

$\begin{array}{ll}\text { 6.5. Conclusions } & 139\end{array}$

6.6. Acknowledgements 141

6.7. References 141

Chapter $7 \quad$ STIMULI RESPONSIVE BORONIC ACID PENTABLOCK COPOLYMERS FOR GLUCOSE RESPONSIVE APPLICATIONS 144

$\begin{array}{lll}\text { 7.1. Abstract } & 144\end{array}$

$\begin{array}{ll}\text { 7.2. Introduction } & 145\end{array}$

$\begin{array}{ll}\text { 7.3. } & \text { Materials and methods } \\ 7.4 .88\end{array}$

$\begin{array}{ll}\text { 7.4. Characterization } & 150\end{array}$

7.5. Results 153

$\begin{array}{lll}\text { 7.6. Conclusions } & 158\end{array}$

$\begin{array}{ll}\text { 7.7. } & 159\end{array}$

CHAPTER 8 THERMOREVERSIBLE PHOTO CROSSLINKABLE

POLYMER GELS FOR POSTTRAUMATIC ARTHRITIS

CARTILAGE HEALING 160

8.1. Abstract 160

8.2. Introduction 161

8.2. Materials and methods 163

8.3. Characterization 166

8.4. Results and discussion 167

$\begin{array}{lll}\text { 8.5. } & 173\end{array}$

CHAPTER 9 FUTURE WORK 175

9.1. General conclusions 175

$\begin{array}{lll}\text { 9.2. } & \text { Future directions } & 176\end{array}$

9.3. References 183

9.4. Acknowledgements 184

APPENDIX Acronyms 


\section{CHAPTER 1. INTRODUCTION}

\subsection{Introduction}

Self-assembly is a powerful tool in forming structures with nanoscale dimensions. Selfassembly of macromolecules provides an efficient and rapid pathway for the formation of structures from the nanometer to micrometer range that are difficult, if not impossible to obtain by conventional lithographic techniques [1]. Depending on the morphologies obtained (size, shape, periodicity, etc.) these self-assembled systems have already been applied or shown to be useful for a number of applications in nanotechnology [2], biomineralization [3, 4], drug delivery [5, 6] and gene therapy [7]. In this respect, amphiphilic block copolymers that self-organize in solution have been found to be very versatile [1]. In recent years, polymer-micellar systems have been designed that are adaptable to their environment and able to respond in a controlled manner to external stimuli. In short, synthesis of "nanoscale objects' that exhibit 'stimulus-responsive' properties is a topic gathering momentum, because their behavior is reminiscent of that exhibited by proteins [8]. By integrating environmentally sensitive homopolymers into amphiphilic block copolymers, smart block copolymers with self assembled supramolecular structures that exhibit stimuli or environmentally responsive properties can be obtained [1].

Several synthetic polymers are known to have environmentally responsive properties. Changes in the physical, chemical or biochemical environment of these polymers results in modulation of the solubility or chain conformation of the polymer [9]. There are many common schemes of engineering stimuli responsive properties into materials $[8,9]$. Polymers exhibiting lower critical solution temperature (LCST) are soluble in solvent below a specific temperature and phase separate from solvent above that temperature while polymers 
exhibiting upper critical solution temperatures (UCST) phase separate below a certain temperature. The solubility of polymers with ionizable moieties depends on the $\mathrm{pH}$ of the solution. Polymers with polyzwitterions, anions and cations have been shown to exhibit $\mathrm{pH}$ responsive self assembly. Other stimuli responsive polymers include glucose sensitive polymers, calcium ion-sensitive polymers and so on. Progress in living radical polymerization (LRP) methods [10] has made it possible for the facile synthesis of these block copolymer systems with controlled molecular weights and well defined architectures.

The overall theme of this work is to develop novel smart block copolymers for biomineralization and biomedical applications. Synthesis and characterization of selfassembling thermoreversible ionic block copolymers as templates in biomimetic nanocomposite synthesis using a bottom-up approach is a novel contribution in this respect. Further, we have extended these families of copolymers to include block copolymer-peptide conjugates to enhance biological specificity. Future directions on this work will focus on enhancing the polymer templating properties for biomineralization by expanding the family of block copolymers with organic polypeptides and biological polypeptide scaffolds as well as a detailed understanding of the polymer-inorganic nanocomposites at the molecular level using small angle scattering analysis. Glucose responsive polymer hydrogels for drug delivery, polymer-ligand conjugates for non-viral therapy and thermoresponsive injectable photocrosslinkable hydrogels for posttraumatic arthritis cartilage healing are other applications of these novel copolymers synthesized in our work.

\subsection{Thesis organization}

The thesis is organized into nine chapters. Chapter 2 is general background of smart block copolymers, their applications in several fields with an emphasis on biomineralization, 
as well as approaches taken in this study to characterize these materials. Potential applications of these smart block copolymers also include in situ photocrosslinkable gels for tissue engineering, cationic vectors for gene therapy and injectable drug depots. The background provides a context for the inspiration and the goals of this work as described in the research objectives in chapter 3 . Chapters 4,5 , and 6 deal with the development of block copolymer templates and the applications of these smart polymers in biomineralization. Chapter 4 is a modified version of a paper published in the Journal of Materials Chemistry that addresses self-assembled polymer calcium phosphate nanocomposite synthesis using block copolymer templates. Chapter 5 is a paper accepted to the Chemistry of Materials on the synthesis and characterization of ionic block copolymer templated calcium phosphate nanocomposites synthesis, while chapter 6 is the expansion of this work to polymer-peptide conjugates (submitted to the Journal of Materials Research). The development of novel block copolymers and applications of these copolymers in the biomedical field is the focus of chapters 7-9. Chapter 7 describes the synthesis and characterization of thermosensitive boronic acid pentablock copolymers for glucose responsive applications, and the work is submitted to the Journal of Biomedical Materials Research. Chapter 8 is a paper in preparation, on thermoreversible photocrosslinkable hydrogels for posttraumatic arthritis cartilage healing. Chapter 9 provides general conclusions of the work and future directions for the development and applications of such families of block copolymers.

\subsection{References}

1. Ikkala, O. and G. ten Brinke, Hierarchical self-assembly in polymeric complexes: towards functional materials. Chemical Communications (Cambridge, United Kingdom), 2004(19): p. 2131-2137.

2. Zhang, S., Fabrication of novel biomaterials through molecular self-assembly. Nature Biotechnology, 2003. 21(10): p. 1171-1178. 
3. Abu-Lebdeh, Y.A.I., P.M. Budd, and V.M. Nace, Preparation of mesoporous silica with poly(oxyethylene)/(oxybutylene)/poly(oxyethylene) triblock copolymers as templates. Journal of Materials Chemistry, 1998. 8(8): p. 1839-1842.

4. Zhao, Y.F. and J. Ma, Triblock co-polymer templating synthesis of mesostructured hydroxyapatite. Microporous and Mesoporous Materials, 2005. 87(2): p. 110-117.

5. Rosler, A., G.W. Vandermeulen, and H.A. Klok, Advanced drug delivery devices via self-assembly of amphiphilic block copolymers. Adv Drug Deliv Rev FIELD Full Journal Title:Advanced drug delivery reviews, 2001. 53(1): p. 95-108.

6. Matsumoto, A., R. Yoshida, and K. Kataoka, Glucose-responsive polymer gel bearing phenylborate derivative as a glucose-sensing moiety operating at the physiological pH. Biomacromolecules, 2004. 5(3): p. 1038-1045.

7. Ogris, M., et al., Tumor-targeted gene therapy: strategies for the preparation of ligand-polyethylene glycol-polyethylenimine/DNA complexes. Journal of Controlled Release, 2003. 91(1-2): p. 173-181.

8. Jeong, B. and A. Gutowska, Lessons from nature: stimuli-responsive polymers and their biomedical applications. Trends in Biotechnology, 2002. 20(7): p. 305-311.

9. Hashidzume, A., Morishima, Y. and Szczubialka, K., Amphiphilic Polyelectrolytes, in Polyelectrolytes and their Applications. American Scientific Publishers:Stevenson Ranch, 2002: p. 2-57.

10. Szwarc, M., Living polymers. Their discovery, characterization, and properties. Journal of Polymer Science, Part A: Polymer Chemistry, 1998. 36(1): p. ix. 


\section{CHAPTER 2. BACKGROUND}

\subsection{Smart block copolymers}

Environmentally sensitive bioinspired smart block copolymers represent a new class of functional materials with tremendous applications in biomineralization and biomedically related fields. By integrating environmentally sensitive homopolymers into amphiphilic block copolymers, self-assembled supramolecular structures that exhibit stimuli or environmentally responsive properties can be obtained $[1,2]$. Hence, the interest in the synthesis and characterization of these families of novel block copolymers has increased enormously in the recent years.

Amphiphilic block copolymers consist of at least two or more covalently linked hydrophilic and hydrophobic macromolecular segments [3]. The hydrophobic blocks of amphiphilic copolymers self-associate in aqueous solutions to form supra molecular aggregates consisting of hydrophobic domains surrounded by swollen hydrophilic blocks. Hydrophobic interactions are a fundamental driving force in the assembly of amphiphilic systems [4]. A unified theory of hydrophobic interactions of small and large apolar species in water have been proposed by Lum and Chandler[5]. Unlike small hydrophobic groups, when larger hydrophobes, such as macromolecules, are introduced the hydrogen bond network has no way of reorganizing to accept the large excluded volume of the apolar species. This results in 'drying out' at the interfaces of the hydrophobic objects where the density of water molecules is highly rarified. These density fluctuations are highly unstable and result in strong attractions between the large hydrophobic objects. This leads to macromolecular self-association and the formation of nanoscale ordered structures. 
Depending on the length of the hydrophilic block, the morphology which originates on the nanoscale can vary from spherical micelles, rods, and vesicles to large compound micelles [1]. In some cases macroscopic gel structures are formed from these nanoscale building blocks. Figure 2.1 shows the structures of some self-organized amphiphilic block copolymers.

The enormous theoretical and technological potential of amphiphilic block copolymers, mainly related to the energetic and structural control of materials interfaces has been well recognized $[2,3]$. By tuning the chemical structure of amphiphilic copolymers, the interfaces with very different chemical nature, polarity, and cohesion energy can be controlled to a broader extent. This is the physical basis for the construction of thermodynamically stable materials hybrids with nanoscale structure. Potential applications of these polymers include, templates for biomineralization [6, 7], drug carriers [6], biosensors [3], molecularly thin membranes [3], and growth of mesoporous inorganic materials [3, 6].

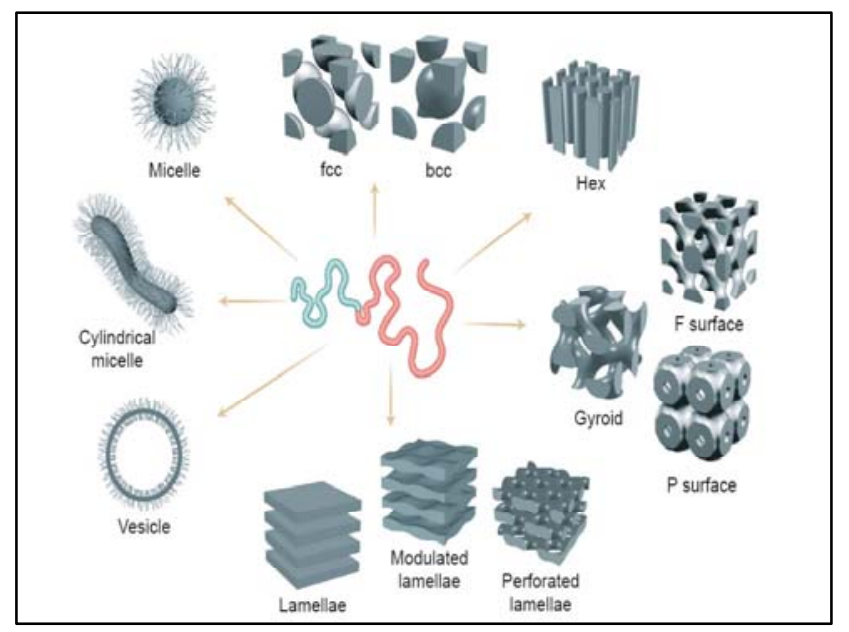

Figure 2.1. Self-assembled polymeric structures of amphiphilic block copolymers (From [3])

\subsection{Self-assembly- micellization and gelation of block copolymers}


Block copolymers self-assemble both in solution and in bulk and are capable of generating a variety of microdomain morphologies due to the covalently bonded blocks with different physical and chemical properties [2]. Amphiphilic block copolymers are the most common block copolymer systems that self-assemble into different morphologies due to their different affinities to certain environments [3]. The two blocks are incompatible and interact differently with their environment due to the chemical nature and also behave distinctively in solution which results in micro phase separation not only in aqueous media but also in organic solvents.

The two basic processes that block copolymers can undergo in solvents are micellization and gelation. Micellization takes place when the block copolymer is dissolved in excess amount of selective solvent for one of the blocks [7]. This in turn leads the polymer chains to organize themselves into variety of structures from spherical micelles or vesicles to cylinders. The hydrophilic blocks will be oriented towards the aqueous solvent medium, and be the corona of the micelle formed while the hydrophobic block will be shielded from the solvent and will be the core part of the structure. On the other hand, in contrast to micellization, gelation occurs from the semi-dilute to the high concentration regime of block copolymer solutions, resulting in an arrangement of ordered micelles [7].

(a)

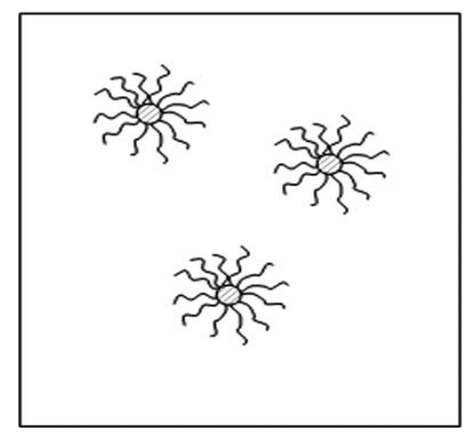

(b)

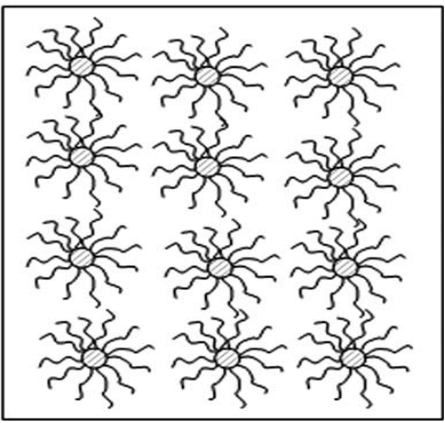


Figure 2.2. llustration of (a) micellization at the $\mathrm{CMC}$ and (b) gelation at high concentration from diblock copolymers (From [2])

\subsubsection{Theoretical aspects}

The micellization process is mainly governed by two parameters, critical micellization temperature (CMT) and critical micellization concentration (CMC) [8]. Self assembly will not occur if either of these is not reached, and the block copolymer will remain as unimers in solution. On the other hand, if micelle formation is triggered, a thermodynamic equilibrium will be reached between micelles and unimers.

Several parameters have to be considered to characterize a micellar system, such as the equilibrium constant, the quality of the solvent, CMT, $\mathrm{CMC}$, the overall mass molecular weight of the micelle, its aggregation number $\mathrm{Z}$ and its morphology [8]. These variables affect the hydrodynamic radius $\mathrm{R}_{\mathrm{H}}$, the radius of gyration $\mathrm{Rg}$, the core radius $\mathrm{Rc}$, and the thickness L of the corona. The shape and size of the aggregates are controlled by a variety of parameters that affect the balance between three major forces acting over the system. The major forces reflect the extent of constraints between the blocks forming the core, the interaction between the chains forming the corona and the surface energy between the solvent and the core of the micelle. A complete description of the aggregate structure requires that the thermodynamic parameters of self-assembly be considered, along with the forces generated between the macromolecules inside the aggregates [2]. The thermodynamics and intra-aggregate forces combined with the inter-aggregate forces between different aggregates determine the self-assembled structure formed at equilibrium. Hence, it is important to understand the fundamentals that govern the interdependence between morphology and size of the aggregates obtained by self-assembly, including essential factors 
such as concentration, temperature, composition, block length, copolymer architecture and the solvents used.

Various theories have been developed to describe the behavior of block copolymers in solution and its dependence on parameters mentioned above [9]. The theories are classified into two main groups, scaling theories and self-consistent mean field theories. Scaling theory is mainly based on the prediction of aggregation number or the radius for crew-cut micelles from the block length and interfacial tension data. The self-consistent mean theory on the other hand is able to predict the size of the spherical micelles at equilibrium, and the variation of the aggregation number as a function of the degree of polymerization. It is mainly based on the molecular characteristics of the polymer, its concentration in solution and an estimation of the core/corona interfacial tension [10], which in good agreement with X-ray and neutron scattering data. The model was expanded to block copolymer systems where it has been found that the size of the micelles, the aggregation number and the fraction of copolymer chains forming the micelles could be calculated. Further development of this theory resulted in its use for other aspects such as evolution of the CMC with block copolymer structure [11], or the temperature dependence of the hydrodynamic radius and aggregation number [12], the transition between spherical and cylindrical micellar systems [12], etc. Recently, the Pederson-core micelle system has been the most useful compendium

of form factors and structure factors for spherical micelles [13]. The Pederson micellar model was a powerful free-form fitting method to determine intra-micellar structure.

\subsubsection{Examples of micellar systems}

Micelles can be classified into several types with morphologies varying from spherical to vesicular or other less common structures, such as inverse micelles, bilayers, or cylinders 
(Figure 2.3). According to Riess [7], the structure of amphiphilic block copolymers in aqueous media can be divided into three classes depending on the nature of the hydrophilic block. Uncharged blocks such as poly(ethylene oxide) (PEO) - also referred to as poly(ethylene glycol) (PEG), positively charged blocks such as quaternized poly(2- or 4vinylpyridine), poly(diethyl-aminomethyl-methacrylate) (PDEAEM), polypeptides such as poly(L-lysine), or negatively charged ones such as poly(acrylic acid) (PAA), poly(styrene sulfonate) (PSS), or poly(L-glutamic acid) (PGA). Recently, Liu et al. report an extensive study on zwitterionic polymer micelles such as poly(sulfobetaines) and poly(phosphobetaines) [14]. The characteristics of these systems make them suitable for applications in biomineralization and biomedical related fields.

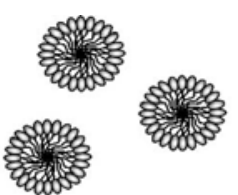

(i)

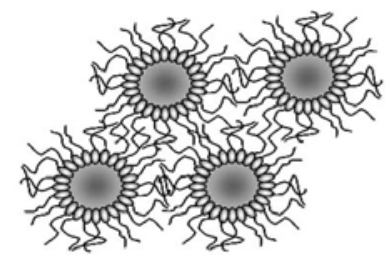

(iii)

(a)

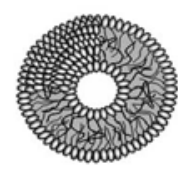

(ii)

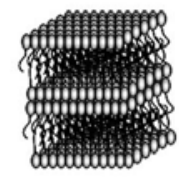

(b)

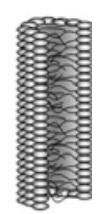

(c)

Figure 2.3. Different types of micellar morphologies of block copolymers: (i) direct micelles (ii) vesicles (iii a) inverse micelles (iii b) lamellar structures, and (iiic) cylindrical of tubular structures (From [2])

Spherical micelles with the so-called 'core-shell' structure have been extensively researched. Spherical micelle formation via self-assembly of diblock copolymers is directed by an entropically driven association mechanism. PEO is a hydrophilic, biocompatible, nontoxic, thermoresponsive polymer, which has been widely used as the solubilizing block to 
form the shell in spherical micelles. Hydrophobic blocks include PS, poly(lactic acid) and polyethers like polypropylene oxide (PPO) or poly(butylene oxide) (PBO). PEO-b-PPO or PEO-b-PBO are commercially available as diblock- (and triblock/ pluronic) have been extensively investigated and Chou and Zhou [15], and the characteristic micellization features of these block copolymers has been reviewed in detail.

Recently Kataoka's group demonstrated that micelles can be excellent vehicles for drug delivery because the drug can be protected from degradation and its deposition site can be better targeted (Figure 2.4). Further by selectivity, the amount of drug administrated can be precisely controlled and thus reduced [6].

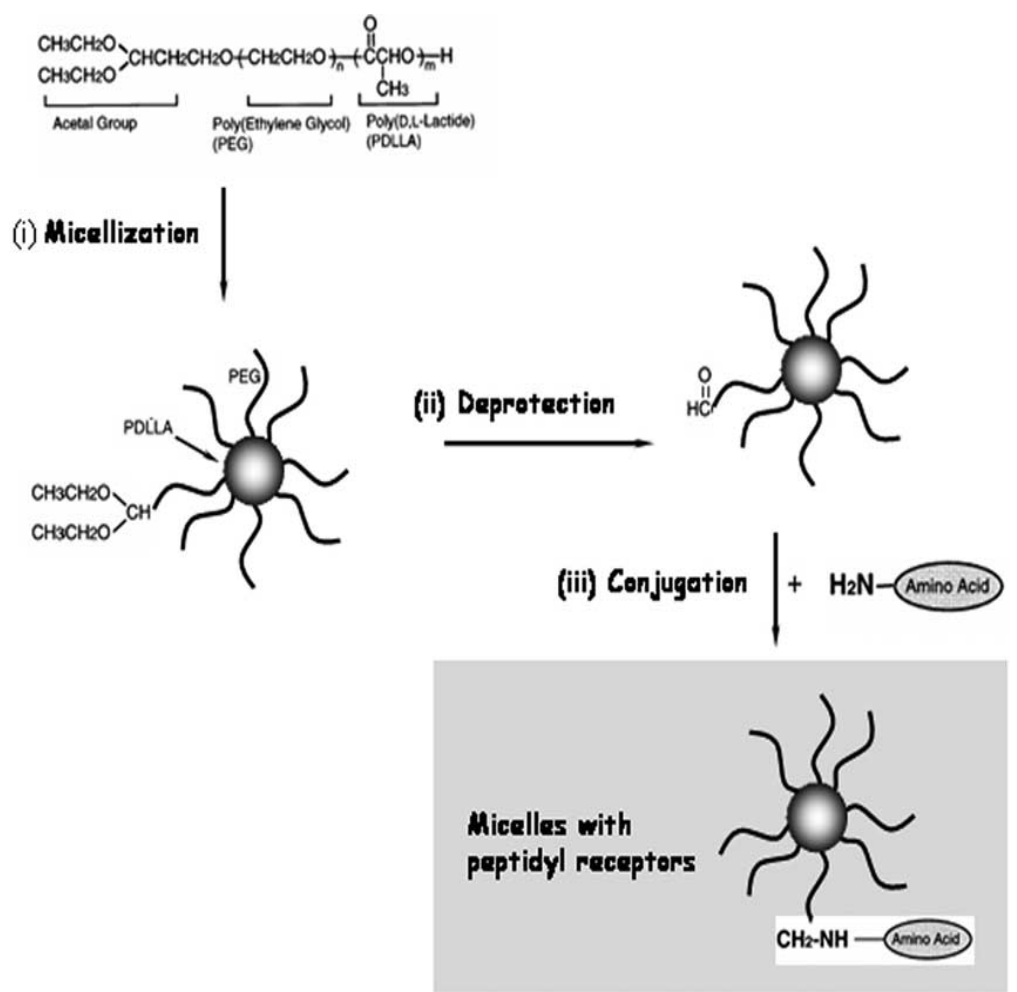

Figure 2.4. Kataoka's approach to the conjugation of amino acids on the micellar surface using PEG-b-PLLA block copolymers. (From [19]) 
The system was based on poly(ethylene oxide)-b-poly( $\beta$-benzylaspartate) (PEOPBLA) block copolymers with PEO as corona. Its small micellar size (diameter $\sim 10$ $100 \mathrm{~nm}$ ) and its long-term stability, which is required for a prolonged circulation time makes it promising as a drug carrier. Further it possesses -OH functional groups in the PBLA block that can be used to easily attach a desired moiety. Other studies exploited the idea of using $\alpha$ acetoxy-poly(ethylene oxide)-b-poly(D,L-lactide) block copolymers [19]. The $\alpha$-acetal group in PEO was transformed into an aldehyde group and conjugated with a peptide segment of phenylalanine (Phe) or tyrosil-glutamic acid (Tyr-Glu).

In addition to common spherical micelles and vesicles, other more complex structures/morphologies based on amphiphilic block copolymers have also been observed. Eisenberg and coworkers reported the formation of cylindrical structures by self-assembly of PS-b-PEO diblock copolymers in aqueous solution [16] or of triblocks in a selective solvent [17]. Similarly Liu et al. were able to prepare aggregates with different morphologies in solution, varying from nanofibers to hollow or 'shaved' nanospheres [18]. Cylindrical structures [19] were obtained by the synthesis of a specifically designed block copolymer with a definite composition. For the preparation of cylinders they used a poly(isoprene-b-(2cinnamomethyl methacrylate)-b-(ter-butyl acrylate)) triblock copolymer with the polyisoprene part forming the core, the poly(2-cinnamoylmethyl methacrylate) (PCMMA) block forming the shell, and poly(tert-butyl acrylate) (PtBA) the corona. Such cylinders generated by self-assembly in methanol exhibited a diameter of $\sim 22 \mathrm{~nm}$, as confirmed by TEM images.

It is well known that secondary structures in synthetic peptides are subject to change from $\alpha$-helix, to coil or to $\beta$-sheet morphology by slight modifications of environmental 
parameters like $\mathrm{pH}$, ionic strength, or temperature. Nolte et al. [20] investigated amphiphilic diblock copolymers based on low molar mass PS and a polypeptidic sequence, the latter being actually a charged helical polyisocyanide block derived from isocyano and alanine or histidine groups. This diblock self-assembles in solution to give micelles, vesicles or bilayers, depending upon the length of the polyisocyanide block, the $\mathrm{pH}$ and the interactions of the anionic head group.

Morphologies other than vesicles involving polyion complexes have been described in the recent literature. For example, Yamamoto et al. [21] made PIC complexes of cationic chitosan and anionic gellan gum, showing that interaction between these charged polysaccharides gives rise either to PIC capsules or to fibers depending on the preparation method (Figure 2.5).

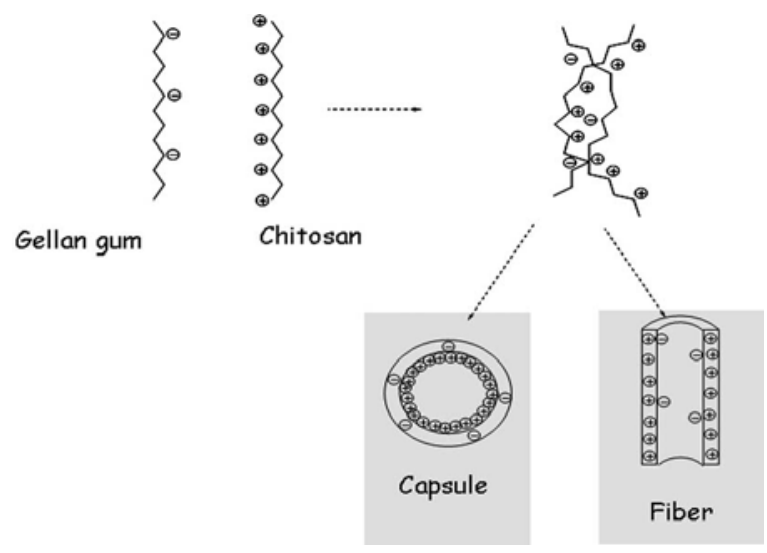

Figure 2.5. Polyion complexes (PIC): interaction between cationic chitosan and anionic gellan for fiber and capsule formation, (From ref [21]).

Interactions of oppositely charged polyelectrolytes have been used for layer-by-layer (LbL) self-assembly to produce interesting hollow spheres or nanocapsules [22],[23]] suitable for applications such as tunable color filters, $\mathrm{pH}$-sensitive coatings, biolabeling, and drug delivery. [24, 25] Möhwald et al. [25] reported a method to prepare capsules from 
colloidal particles using LbL techniques and to remove the colloidal core. The hollow polyelectrolyte capsules obtained were dependent on the physical and chemical conditions of the environment. Zhang et al. [26] fabricated hollow capsules using H-bond interactions instead of strong polyelectrolyte forces. These interactions showed high stability, and the cores of the intermediate capsules could be removed by treatment with HF while the multilayer shell remained intact.

\subsection{Stimuli-responsive block copolymers}

Stimuli-responsive polymers exhibit large, sharp changes to physical stimuli (such as temperature, solvents, or light) or to chemical stimuli such as reactants, $\mathrm{pH}$, ions in solution, or chemical recognition. The response depends on the stimulus applied and may include changes in shape, volume, mechanical properties, or permeation rates. Several synthetic polymers are known to have environmental responsive properties. Changes in the physical, chemical or biochemical environment of these polymers results in modulation of the solubility or chain conformation of the polymer [27]. There are many common schemes of engineering stimuli responsive properties into materials [28, 29]. Polymers with lower critical solution temperature (LCST) are soluble in water below a specific temperature and phase separate from water above that temperature while polymers with upper critical solution temperature (UCST) phase separate below a certain temperature. The solubility of polymers with ionizable moieties depends on $\mathrm{pH}$ of the solution. Polymers with polyzwitterions, polycations and polyanioins are capable of imparting $\mathrm{pH}$ sensitive properties. Polymers that are responsive to external stimuli such as glucose or calcium ion sensitivity are capable of stimulant reactive responsiveness.

\subsection{1. $\mathrm{pH}$ responsive block copolymers}


Several block copolymers that undergo changes with $\mathrm{pH}$ variation have been investigated. Ionizable polymers with a $\mathrm{pKa}$ value between 2-11 are candidates for $\mathrm{pH}$ responsive systems [30]. Classical monomers are acrylic acid (AA), methacrylic acid (MAA), maleic anhydride (MA) and N, N-dimethylaminoethyl methacrylate (DMAEMA).

Block copolymers that contain weak basic or acidic blocks with different pKa values form $\mathrm{pH}$ induced micellization. Here, the copolymer exists as unimers at certain critical $\mathrm{pH}$ but forms micelles when the $\mathrm{pH}$ is changed. Armes and coworkers [32, 33] first reported such systems of zwitterionic diblock copolymers that exhibit dual $\mathrm{pH}$ responsive self assembly. Figure 2.6 illustrates some of the zwitterionic homopolymer structures.

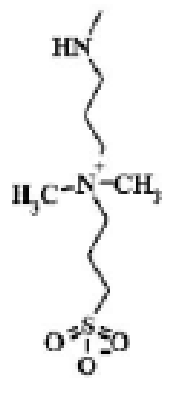

(a)

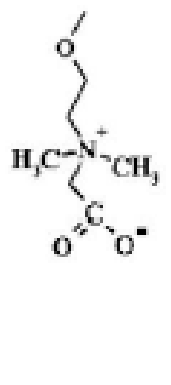

(b)

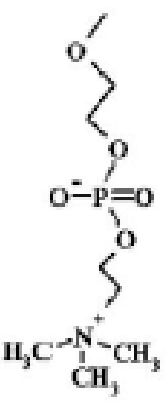

(c)

Figure 2.6. Examples of zwitterionic homopolymers (a) sulfobetaine (b) carboxybetaine, and (c) phosphobetaine (adapted from [14] ).

Liu and Armes prepared a poly(4-vinyl benzoic acid-b-2-(diethylamino)ethyl methacrylate (VBA-DEA) copolymer by ATRP using protecting group chemistry[31] Subsequently the ATRP synthesis of zwitterionic diblock copolymers without the use of protecting group chemistry has been reported [32]. The polybasic DEA and the polyacidic VBA have pKa values of 7.3 and 7.1 respectively. At low $\mathrm{pH}$, the uncharged VBA block is hydrophobic. Because the DEA block is highly charged and hydrophilic at this $\mathrm{pH}$ the copolymer forms well defined micelles with VBA cores and DEA coronas. Because both 
blocks are uncharged and hydrophobic in a $\mathrm{pH}$ range around the isoelectric point of the copolymer, between $\mathrm{pH} 6.8$ and 8.3 , the copolymer precipitates. This is observed by an increase in turbidity of the solution, and can be attributed to the formation of large nonmicellar aggregates [32] Above $\mathrm{pH} 8.3$ the VBA groups become charged and hydrophilic, resulting in formation of DEA-core, VBA-corona micelles. Figure 2.7 illustrates some of the homopolymers that impart $\mathrm{pH}$ sensitivity at high $\mathrm{pH}$.

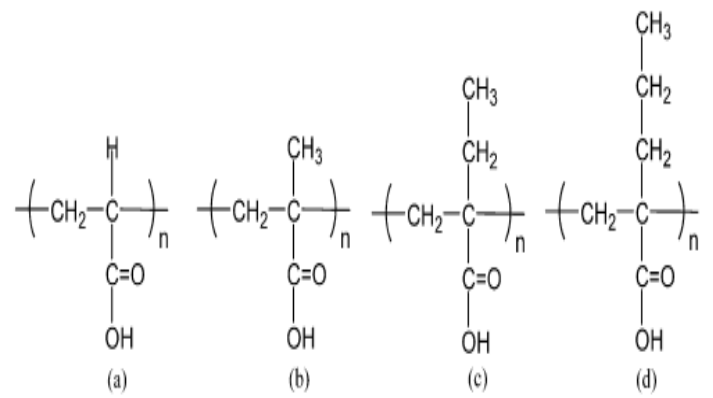

Figure 2.7. Examples of anionic polymer structures (a) poly(acrylic acid) (b) poly (methacrylic acid) (c) poly(2-ethyl acrylic acid) (d) poly(2-propyl acrylic acid) (adapted from [30]).

The same authors recently reported a similar $\mathrm{pH}$ responsive system of PEO-DEA copolymer co-dissolved with PMMA homopolymer [33]. Four distinct, $\mathrm{pH}$ dependent micellar and polyionic complexes were identified; PEO/PMAA-core, PDE corona micelles at low pH, a DEA-PMAA complex stabilized in solution by the hydrophilic PEO block with increasing $\mathrm{pH}$, micelles formed with neutral DEA-PMMA complex cores and PEO coronas, and at high $\mathrm{pH}$ PDEA-core, PEO-corona micelles formed, excluding the hydrophilic PMMA homopolymer to the solution phase [33].

The $\mathrm{pH}$ dependent, reversible micelle formation of polyelectrolyte block copolymer poly(N,N' diethyleaminoethyl methacrylate)-b-poly(N,N'-dimethylaminoethyl methacrylate) (PDEA-b-PDMA) were characterized with light and small angle neutron scattering[34]. 
Because both PDEA and PDMA blocks are polybasic the copolymer is a highly charged polyelectrolyte at low $\mathrm{pH}$. Increasing the $\mathrm{pH}$ of the copolymer solution results in deprotonation of the tertiary amine pendents of both blocks. Onset of micelle formation occurred when just over half of each block was deprotonated. The more hydrophobic PDEA blocks aggregated to form a core domain surrounded by hydrophilic PDMA. The $\mathrm{pH}-$ dependent micelle formation was designed to be capable of encapsulating drugs during the micellization process, followed by drug release when the micelle dissolved in a low $\mathrm{pH}$ environment. The triblock copolymer polystyrene-b-poly(2-vinylpyridine)-b-poly(ethylene oxide), which forms three layered micelles, showed $\mathrm{pH}$ sensitivity below $\mathrm{pH} 5$ due to the ionization dependency of poly(2-vinylpyridine). The P2VP block swells at low $\mathrm{pH}$ due to protonation, which makes the system useful for encapsulation. Figure 2.8 illustrates some of the widely used $\mathrm{pH}$ responsive cationic homopolymers in biotechnological applications.

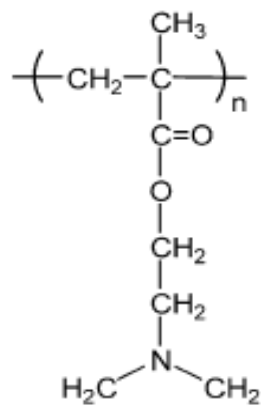

(a)

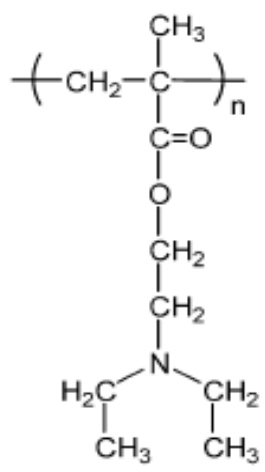

(b)

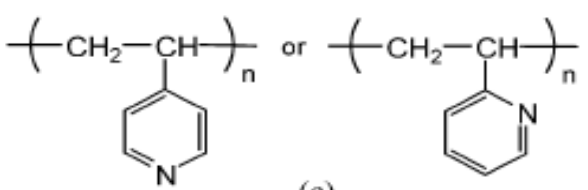

(c)

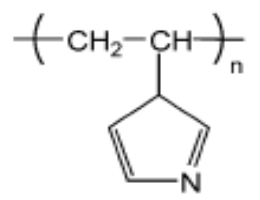

(d)

Figure 2.8. Examples of cationic polymer structures (a) poly(N,N'-dimethyl aminoethyl methacrylate) (PDMAEMA) (b) poly(N,N'-diethyl aminoethyl methacrylate) (PDEAEMA) (c) poly(4 or 2-vinylpyridine) (PVP) (d) poly(vinyl imidazole) (adapted from [30])

\subsubsection{Temperature responsive block copolymers}


Poly(N-isopropylacrylamide) (PNIPAAm) undergoes a reversible volume phase transition caused by the coil to globule transition at the LCST[35]. The LCST of PNIPAAm can be increased by copolymerizing with a hydrophilic monomer, such as acryl amide; and decreased by copolymerization with a hydrophobic monomer, such as N-butyl-acrylamide. The LCST behavior of poly(ethylene oxide) and poly(propylene oxide) originates from temperature dependent intermolecular interactions in water. The number of hydrogen bonds a PEO or PPO monomer unit is capable of supporting decreases with increasing temperature. Above the LCST, PPO is significantly more hydrophobic than PEO due to the extra methyl group in the repeating unit. The LCST of PPO occurs between $2-8^{\circ} \mathrm{C}$ and decreases with increasing molecular weight. PPO with molecular weight greater than 4000 daltons does not exhibit a LCST [36]. The LCST of PEO increases with increasing molecular weight between 70 and $91^{\circ} \mathrm{C}$. In addition PEO has been found to have an in vivo "stealth" property- it is does not react negatively with immune system. Recent reviews by Gil and Hoffman contain detailed discussions of these families of polymers with LCST in aqueous environment. [30, $31]$.

A temperature sensitive diblock copolymer, combining blocks exhibiting LCST and UCST was recently reported. A copolymer of PNIPAM and a zwitterionic monomer 3-[N(3-methacrylamidopropyl)-N,N-dimethyl]ammoniopropane sulfonate (SSP) was synthesized by sequential RAFT polymerization [40] PNIPAM has a well characterized LCST and P(SSP) exhibits a UCST due to strong mutual intermolecular attraction of zwitterionic groups. This results in a dual thermoresponsive hydrophilic copolymer. Below the UCST of the $\mathrm{P}(\mathrm{SSP})$ the copolymer forms micelles with $\mathrm{P}(\mathrm{SSP})$ cores surrounded by PNIPAM coronas. At intermediate temperatures both blocks are hydrophilic and the copolymer is 
molecularly dissolved. Above the LCST of PNIPAM, micelles form with collapsed PNIPAM cores and swollen $\mathrm{P}(\mathrm{SSP})$ coronas. Unlike the native homopolymers, the copolymer remains in solution between 0 and $100^{\circ} \mathrm{C}$.

Copolymers that undergo temperature and $\mathrm{pH}$ responsive self assembly are formed by combining a polyelectrolyte block and a nonionic block exhibiting a LCST. A diblock copolymer of PPO and DEA was synthesized by ATRP[31]. The LCST of the PPO block $(\mathrm{Mn}=2000)$ was $20^{\circ} \mathrm{C}$. At low $\mathrm{pH}$, where the DEA block is hydrophilic, the copolymer could be dissolved as unimers below $20^{\circ} \mathrm{C}$, or form PPO core micelles above $20^{\circ} \mathrm{C}$. DEA core micelles could be formed at high $\mathrm{pH}$ and temperatures below $20^{\circ} \mathrm{C}$. A copolymer of 2(dimethylamino)ethyl methacrylate-b-2(N-morpholino)ethyl methacrylate (PDMA-bPMEMA) was synthesized via group transfer polymerization[37]. The DMA block was converted to a sulfobetaine methacrylate by selectively quaternizing with 1,3-propane sultone. The zwitterionic poly(sulfobetaine methacrylate) (PSBMA) is soluble above $25^{\circ} \mathrm{C}$, whereas the PMEMA has a LCST between 34 and $49^{\circ} \mathrm{C}$. This copolymer formed micelles with PSBMA cores at low temperature, dissolved molecularly at intermediate temperatures (between 25 and $50^{\circ} \mathrm{C}$ as determined by light scattering), and formed 'reverse' micelles with PMEMA cores. Static light scattering revealed that near monodisperse micelles formed were 42 and $50 \mathrm{~nm}$ in diameter at low and high temperatures respectively. Figure 2.9 shows some of the thermo-reversible polymer structures. 

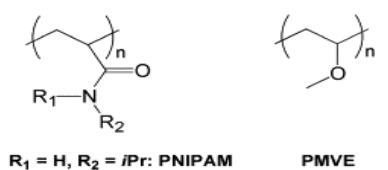

$\mathbf{R}_{1}=\mathbf{H}, \mathbf{R}_{2}=$ PPr: PNIPAM
$\mathbf{R}_{1}=\mathbf{R}_{2}=$ Et: PDEAAm

PMVE
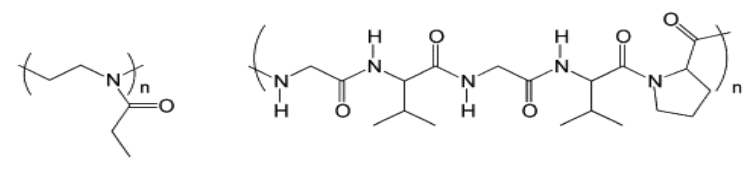

PEtox

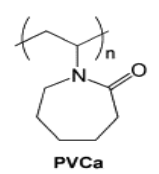

P(GVGVP)

Figure 2.9.Examples of thermoreversible homopolymers used in various biomedical applications (From [30]).

\subsubsection{Glucose responsive block copolymers}

Recently, boronic acid based glucose-responsive copolymers have shown lots of promise [38, 39]. A block copolymer containing poly(3-acrylamidophenylboronic acid), poly (AAPBA)-b-poly(DMAEMA) showed glucose sensitve reponsiveness due to the protonation of AAPBA in the presence of glucose. AAPBA, which is a phenyl boronic acid compound, is known to form covalent complexes with glucose. Since in aqueous medium these AAPBA compounds are in equilibrium as charged and uncharged forms (Figure 2.10), the complexation of glucose is able to shift the equilibrium towards the direction of increased charge of phenylborates, since only charged borates can form complexes with glucose in aqueous media.
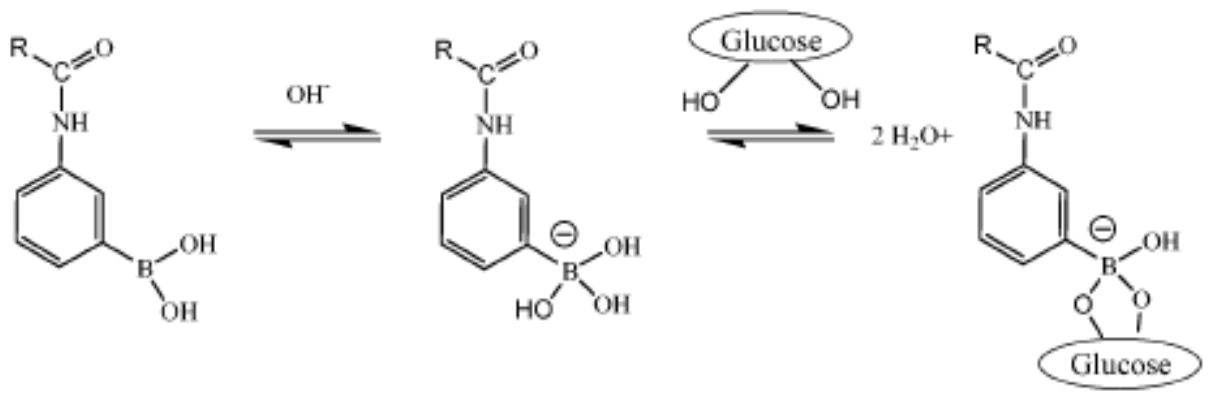

Figure 2.10. Representation of complexation of AAPBA and glucose in aqueous solution (From [39]) 


\subsubsection{Other stimuli responsive and multi-responsive block copolymers}

Several examples of self-assembled structures with multi-stimuli responsive aptitude have been described in the literature. For instance, Armes et al. described 'schizophrenic' diblock copolymers that form direct and inverse micelles in the same solvent. They prepared different systems exhibiting such behavior. One was based on a diblock copolymer weak polybases: poly[2-( $N$-morpholino) ethyl methacrylate-b-2-(diethylamino)ethylmethacrylate] (MEMA-b-DEAEMA). DEAEMA cores were formed by adjusting the $\mathrm{pH}$ of the solution [40]. In addition to the response to $\mathrm{pH}$ variation, such a system is also sensitive to polyelectrolytes, which can reverse the micelle, i.e. with the MEMA block forming the core. The second example concerns PPO-b-DEA block copolymers [31]. PPO is a thermoresponsive block and DEA a $\mathrm{pH}$ responsive block. Micelles with a DEA core were obtained in solution at low temperature and alkaline $\mathrm{pH}$. After raising the temperature to $40-$ $70{ }^{\circ} \mathrm{C}$ and decreasing the $\mathrm{pH}$ to 6.5 , the reverse situation was observed with PPO forming the core. Recently, Armes et al. [40] described a more complex zwitterionic diblock of poly[4(vinylbenzoic acid)-b-2-( $N$-morpholino)ethyl methacrylate] (VBA-b-MEMA) that can respond to $\mathrm{pH}$, ionic strength, and temperature, and form normal as well as inverse micelles. At low $\mathrm{pH}$ they obtained micelles with a VBA core and at high $\mathrm{pH}$ and in the presence of salt, or at high temperatures, micelles with a MEMA core. P2VP has been used by several groups as a $\mathrm{pH}$ responsive block. Another example is the formation of 'schizophrenic' vesicles obtained from the self assembly of diblock copolypeptides poly(glutamic acid)-bpoly(1-lysine) PGA-b-PLys [41]. This system is able to reversibly form vesicles, free chains in solution and reversed vesicles upon $\mathrm{pH}$ variations. Gohy et al. [46] also reported stimulusresponsive micelles from an ABC triblock copolymer based on PS-b-P2VP-b-PEO. The PS- 
b-P2VP and the PEO blocks were connected by a stable metal-complex of bis $\left(2,2^{\prime}: 6^{\prime}, 2^{\prime \prime}-\right.$ terpyridine) ruthenium (II) that retained its integrity under extreme conditions of $\mathrm{pH}$, temperature, and salt concentration. The use of such a complex imparted new properties such as the reversibility of the complex formed under certain conditions, and additional photophysical and electrochemical properties. Micelles of these triblocks were obtained at low $\mathrm{pH}$ upon deprotonation of the P2VP block. At neutral $\mathrm{pH}$ the P2VP block shrank, entailing shrinkage of the aggregates around $60-80 \mathrm{~nm}$. In addition, variations in temperature from 20 to $70{ }^{\circ} \mathrm{C}$ provoked a decrease of $R_{\mathrm{H}}$ as well. Unfortunately, neither the $\mathrm{pH}$ nor the temperature responses were reversible.

Finally, a versatile system was proposed by Nowakowska and coworkers [42], who synthesized a series of amphiphilic terpolymers based on sodium 2-acrylamido-2-methyl-1propanesulfonate (AMPS), N-isopropylacrylamide (NIPAM) and cinnamooyloxyethylmethacrylate (CEMA). The terpolymers were soluble in water, prone to selfassemble into micelles and sensitive to three stimuli: (a) temperature, due to the NIPAM block that imposed a lower critical solution temperature (LCST), (b) UV-light, due to the presence of the cinnamoyl block, and finally (c) ionic strength, that at elevated concentration provoked loss of the temperature-sensitivity. The size of the micelles formed, as indicated by dynamic light scattering (DLS), was found to vary with temperature and UV irradiation.

\subsection{Hydrogels}

\subsubsection{Stimuli responsive hydrogels}

Hydrophilic polymers, and especially their crosslinked forms, known as hydrogels, are a class of biomaterials that have demonstrated great potential for biological and medical applications [43]. Recently, hybrid materials have been developed to preserve the bulk 
properties of traditional polymers while making their molecular chains look more like proteins [44]. The advantage of using synthetic polymeric materials based solely on proteins or peptides is that it offers a high degree of control over properties. Peptides and proteins can be coded for specific properties using a basic knowledge of inter-and intra chain interactions. Both the traditional and protein based hydrogels have been generated with a variable degree of efficiency and complexity [43]. These hydrophilic polymer networks have a high affinity for water but are prevented from dissolving due to their chemically or physically crosslinked network. Water can penetrate in between the polymer chains of the polymer network, subsequently causing swelling and the dissolution of the hydrogel. Because of their high water content and biocompatibility hydrogels have attracted a great deal of attention, and many progresses have been made in using these materials for many biological and biomedical applications.

The hydrogel networks are characterized using parameters such as polymer volume fraction in the swollen state $(t 2, \mathrm{~s})$, the molecular weight of the polymer chain between two neighboring crosslinking points $(M c)$, and the corresponding mesh size $(n)$ [43]. The amount of fluid imbibed and retained by the hydrogel in the swollen state is measured by polymer volume. The molecular weight between two consecutive cross-links, which can be either chemical or physical in nature, is a measure of the degree of crosslinking of the polymer. Due to the random nature of most polymerization processes, typically only average values of $M c$ can be calculated. The correlation length or distance between two adjacent crosslinks, $n$, provides a measure of the space available between the macromolecular chains (e.g., for drug diffusion). These parameters, which are related to one another, can be determined 
theoretically or through the use of a variety of experimental techniques such as equilibriumswelling and rubber-elasticity theory [43].

By tailoring their molecular structure, polymer networks can be created that have stimuli responsive properties. Recently extensive research focused on the development and application of new environmentally sensitive hydrogels, especially those sensitive to temperature, $\mathrm{pH}$, and specific analytes [45-47]. The response mechanism is based on the chemical structure of the polymer network such as the functionality of chain side groups, branches, and crosslinks. In networks that contain weakly acidic or basic pendent groups, waters of sorption can result in ionization of these pendent groups depending on the solution $\mathrm{pH}$ and ionic composition. The gels in turn act as semi permeable membranes for the counter ions, hence influencing the osmotic balance between the hydrogel and the external solution through ion exchange, depending on the ion-ion interactions. For ionic gels containing weakly basic pendent groups, the equilibrium degree of swelling increases as the $\mathrm{pH}$ of the external solution decreases, while the degree of swelling increases as the $\mathrm{pH}$ increases for gels containing weakly acidic pendent groups.

Some of the commonly studied ionic polymers include poly(acrylic acid), poly(methacrylic acid), polyacrylamide(PAAm), poly(diethylaminoethylmethacrylate), and poly(di-methylaminoethylmethacrylate). Polyacidic hydrogels have been developed for oral administration of insulin. The hydrogel is collapsed at low $\mathrm{pH}$ values and entraps the insulin hence protecting it protein from the harsh environment of the upper gastro-intestinal track. The hydrogel swells and the release of the insulin is triggered by the increase in the environmental $\mathrm{pH}$ of the small intestine $[55,56]$ Another example is the diffusion transport of streptokinase from crosslinked hydrogels of poly(hydroxyethyl methacrylate)-co- 
poly(diethyl aminoethyl methacrylate) (PHEMA-co-PDEAEM) [48]. Around neutral pH, the tertiary amine pendents are unionized and the hydrogel is collapsed, inhibiting diffusion of drug out of the hydrogel network. When the $\mathrm{pH}$ is lowered the PDEAEM gets protonated, swelling the hydrogel network and increasing the effective rate of drug diffusion out of the gel.

Temperature- responsive hydrogels are one of the versatile responsive hydrogel systems. Networks showing a lower critical miscibility temperature tend to shrink or collapse as the temperature is increased above the LCST, and the gels swell up on lowering the temperature below the LCST. The system behaves in an opposite fashion for upper critical solution systems (UCST). Aqueous solutions of amphiphilic triblock copolymers of poly(oxyethylene)-block-poly(oxypropylene)-block-poly(oxyethylene) PEO-b-PPO-b-PEO, known by trade names of Pluronic ${ }^{\circledR}$ and Poloxemer ${ }^{\circledR}$, exhibit thermo reversible phase behavior [36]. The LCST behavior of PPO, guides the triblock copolymer to thermo reversible gelation transition under environmental conditions. Another system which are mostly based on poly( $N$-isopropylacrylamide) (PNIPAAm) and its derivatives, undergo a reversible volume-phase transition with a change in the temperature of the environmental conditions [35]. PNIPAAm exhibits a LCST around $33^{\circ} \mathrm{C}$. Solutions of poly(ethylene oxideb-(DL-lactic acid-co-glycolic acid)-b-ethylene oxide) (PEG-PLGA-PEG) copolymers form micelles and soft solid gel phase upon warming [58]. The biodegradable PLGA block is four times as hydrophobic as the PPO block of Pluronic ${ }^{\circledR}$ copolymers. Drug formulations including this material have been shown to be highly biocompatible [49].

Theromosensitive photopolymerizable hydrogels with crosslinked network is of interest in applications that require injectable formulations with in situ macroscopic gelation, 
high moduli gels and prevent dissolution of the gel in an aqueous environment. Several crosslinking methods to design hydrogels have been developed over the years [50-52]. Recently polymers with vinylmonomers were used to design crosslinked polymer gel networks [52-54]. The vinyl macromers mainly contain methacrylate or acrylate groups, which are capable of forming crosslinking networks within minutes in the presence of UV light [55]. This photopolymerization has advantage over other methods, especially in tissue engineering related applications, where in situ polymerization can be realized as well as less toxic compared to other crosslinking methods[51, 52].

\subsubsection{Artificial polypeptide hydrogels}

Designing artificial hydrogels assembly through naturally occurring protein motifs as well as synthetic polypeptides have opened new pathways in creating hydrogel structures with unique properties $[44,56,57]$. Nature has given a diverse repertoire of protein sequences that could be used in designing various macromolecules with intrinsic properties. This biomimic hydrogel scaffolds have the advantage over the naturally occurring biopolymers, due to the flexibility in designing the structure depending on the end needs, hence limiting the complex nature of the biopolymer structural features.

Protein polymer scaffolds has the advantage over synthetic peptide scaffolds, where the sequence control and molecular weight distribution are hard to control in the latter. Recently, Tirrell and coworkers reported that, artificial protein hydrogels containing leucine zippers are capable of forming self-assembled networks through inter-chain crosslinking and are stimuli responsive to $\mathrm{pH}$ and temperature [56]. Further an elastin-mimetic domain containing a pentapeptide sequence VPGVG, forms a homogenous film upon crosslinking and exhibits mechanical properties similar to arterial wall [58]. More recently, the protein 
polymer structures are further engineered to produce structures with novel chemical, physical and biological properties by the incorporation of noncanonical aminoacids [59, 60]. Some examples include the incorporation of fluorinated aminoacid anologs to increase the melting temperature of coiled-coild domains and collagen-like triple helices, reactive azide groups to post-translationally modify newly synthesized protein containg azedophenylalanine and in photocrosslinking modifications [61-63]. The main disadvantage of protein polymer design is that the choice of monomers is limited to the 20 aminoacids as well as the toxicity.

Organic polypeptides on the other hand have more flexibility in chosing the monomers for the synthetic polymer scaffold design. Initial work on synthetic polypeptide scaffolds focused on poly(L-lysine) and poly(L-aspartic acid) water-soluble homopolypeptides $[44,64]$. Recently Deming and coworkers have introduced facile synthesis of polypeptide hydrogels scaffolds using NCA polymerization. The hydrogel formation was discovered in diblock copolypetides with water solubilizing (poly(L-lysine), K) or (poly (Lglutamate), E) domain and a $\alpha$-helical hydrophobic domain of (poly(L-leucine), L) representing KmLn or EmLn where $\mathrm{m}$ and $\mathrm{n}$ represents the blocks of each aminoacids [44]. These polypeptides self-assemble through the interchain repulsions of the polyelectrolyte chains and the packing of hydrophobic helical segments into fibrillar tapes to minimize overall energy [44]. The polypeptides were capable of forming gels at concentrations low as $0.1 \mathrm{wt} \%$ with the moduli values in the range of $100-500 \mathrm{~Pa}$. Stronger gel structures were produced when the hydrophobic blocks were increased [64]. These polypeptides have various medical applications such as polypeptide membranes in drug delivery and polypeptide adhesives to form moisture-resistant adhesive bonds as well as antimicrobial and immunomodulating applications [57]. 


\subsection{Polymer synthesis methods}

\subsubsection{Living radical polymerization (LRP)}

Free radical polymerization has gained much attention in the field of industrial polymer synthesis due to its simplicity, compatibility and convenience [65]. The major limitation of conventional radical polymerization is its characteristically broad molecular weight distribution of the resulting polymers. This limitation is mainly due to the termination process between 2 propagating radicals. Until recently, ionic polymerization was the only practical route towards block copolymers with controlled molecular weight and architecture [66]. Since ionic synthesis techniques can not be applied to many functional monomers and require rigorous exclusion of water and oxygen, LRP techniques have been utilized synthesize many stimuli responsive copolymers with various controlled architectures [67]. The general feature of these techniques is the use of reagents which convert chain propagating radicals into a "dormant" form in equilibrium with the "active" form.

Among the LRP techniques have been developed, atom transfer radical polymerization (ATRP), reversible addition fragmentation transfer (RAFT) polymerization, and nitroxide mediated polymerization (NMP) are the most common [68]. In these techniques, the main feature is the dynamic equilibrium between actively propagating radicals and dormant polymer chains. Further, the reaction conditions must be selected such that the dormant species is favored in the equilibrium which results in persistent, low concentrations, of propagating radicals. The normal radical termination reactions are effectively suppressed by the low concentration of propagating radical species. Each technique differs primarily in the chemistry of the cap on the dormant polymer chain. The 
ATPR, NMP and RAFT techniques utilize $\omega$-halide, $\omega$-alkoxamine and $\omega$-dithioester caps respectively and ATRP seems to be the most important of the LRP techniques.

\subsubsection{Atom Transfer Radical Polymerization (ATRP)}

Atom transfer radical polymerization is a controlled/“living” polymerization based on the use of radical polymerization to convert monomer to polymer. Matyjaszewski's research group was the first to develop a controlled/“living” polymerization that used a simple, inexpensive polymerization system [59-61]. ATRP is capable of polymerizing a wide variety of monomers, is tolerant of trace impurities (water, oxygen, inhibitor), and is readily applicable to industrial processes. Due to its robustness, ATRP technique has generated much interest among polymer chemists both in industry and academia. The control of the polymerization afforded by ATRP is a result of the formation of radicals that can grow, but are reversibly deactivated to form dormant species (Figure 2.11). Reactivation of the dormant species allows for the polymer chains to grow again, only to be deactivated later. Such a process results in a polymer chain that slowly, but steadily, grows and has a well-defined end group (for ATRP that end group is usually an alkyl halide).

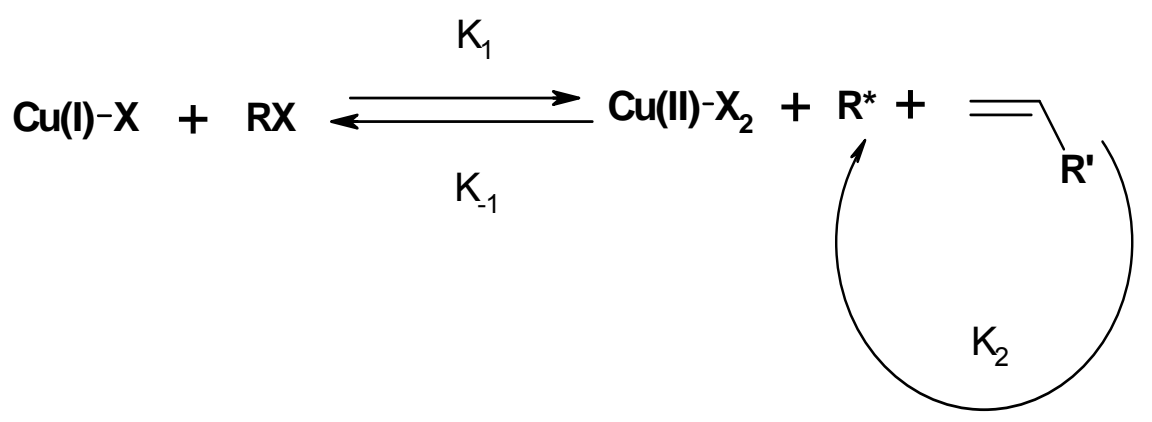

Figure 2.11. ATRP reaction mechanism (adapted from [69])

The initiator is generally a simple alkyl halide. The catalyst is a transition metal that is complexed by one or more ligands and can be used with the initiator in smaller amounts. 
The deactivator can be formed in situ, or for better control, a small amount (relative to the catalyst) can be added. Further, the catalyst is tolerant of water and trace amounts of oxygen and simple transition metals (iron, copper) are used as the catalysts. Compared to other polymerization systems, ATRP requires very small amount of catalyst.

Although other controlled radical polymerization systems have been reported by various groups [65-69], ATRP remains the most powerful, versatile, simple, and inexpensive method. Further, only ATRP has been able to polymerize a wide range of monomers including various styrenes, acrylates and methacrylates as well as other monomers such as acrylonitrile, vinyl pyridine, and dienes [69].

\subsubsection{Reversible Addition-Fragmentation chain Transfer (RAFT)}

Reversible addition-fragmentation chain transfer (RAFT) polymerization allows the synthetic tailoring of macromolecules with complex architectures including block, graft, comb, and star structures with predetermined molecular weight, terminal functionality, and narrow molecular weight distribution [70].

RAFT polymerization is the most recent living radical polymerization technique after nitroxide-mediated polymerization and atom transfer radical polymerization (ATRP) [71]. The free radical polymerization shows living characteristics in the presence of RAFT agent by reversible addition and fragmentation chain transfer process. Using RAFT polymerization, homopolymers, block copolymers, branched and gradient polymers with narrow polydispersities can easily be prepared [72]. Further, it is totally compatible with conventional free radical polymerization. The main advantage of RAFT compared to ATRP and NMP polymerization is the wide range of monomers that could be easily polymerized by this method, mainly carboxyl monomers [60-62]. 


\subsubsection{N-Carboxy Anhydride (NCA) polymerization}

The polymerization of $\alpha$-aminoacid-N-carboxyanhydrides (NCAs) are the most efficient synthesis of long polypeptide chains $[73,74]$. Figure 2.5.4.1 illustrates the basic ring opening mechanism of the NCA polymerization [74]. NCA polymerization utilizes simplest reagents and facilitates high molecular weight polymers in high yield with high purity with minimal racemization at the chiral centers [75]. This polymerization techniques is a good alternative for large molecular weight polypeptide compared to the conventional solid-phase peptide synthesis techniques where large polypeptides ( $>100$ residues) preparation is not possible due to the deletions and truncations during the deprotection and coupling steps [75].
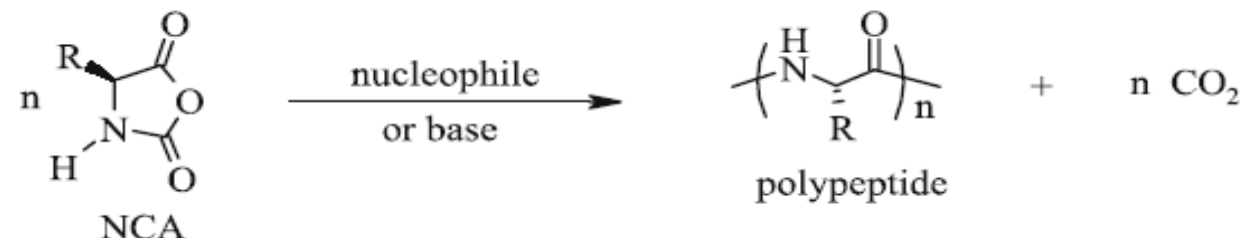

Figure 2.5.4.1. Simple NCA ring opening polymerization mechanism (From [75])

\subsection{Characterization of smart copolymer supramolecular self assembly}

Since the length scale of supramolecular self assembly of block copolymers (from unimers to micelles and gels) spans several order of magnitudes a wide variety of techniques are needed to characterize them [27]. Nuclear Magnetic Resonance (NMR) is used to find the degree of polymerization by using end group analysis. Gel Permeation Chromatography (GPC) can be used to find the molecular weight and polydispersity of the polymer. Cryogenic transmission electron microscopy can be employed to directly visualize the micellar structures of copolymers by preserving the self assembled structures present in the ambient conditions in the near future if needed. Laser light scattering can be applied to 
characterize weight average molecular weights and radius of gyration of the polymer macromolecules; further they also can be used to determine the size and weight of the polymer micellar solutions. Small angle scattering, both X-ray and neutron (SAXS and SANS) can be used to investigate polymers in solutions and in periodic gel and solid networks by utilizing the inhomogenities of the scattering length density of the polymer sample. Further, to understand the mechanical, thermal and rheological properties of smart copolymer gels, solids and micellar morphologies, techniques such as dynamic mechanical analysis (DMA), rheological studies and differential scanning calorimetry (DSC) can be used.

\subsubsection{Spectroscopic techniques}

NMR technique is used to find the compositions of the synthesized polymer. Regarding the fluidity of the polymer core micelles, NMR provides information on the mobility of the polymer chains in their micelle form. Numerous studies have utilized NMR to study the phase separation induced by changes in either $\mathrm{pH}$ or temperature $[63,64]$. FTIR, which is a powerful tool for characterizing and identifying organic molecules, can be used to track the changes in hydrogen bonding and the hydration of particular bonds of a polymeric material. Other spectroscopic techniques such as UV-vis, fluorescence spectroscopy could be used to probe molecules that are selectively solvated into microdomains formed by amphiphilic block copolymers, to investigate self assembly [70, 71]

\subsubsection{Separation techniques}

Gel permeation chromatography (GPC), also called size exclusion chromatography and gel filtration, affords a rapid method for the separation of oligomeric and polymeric species. The technique is based on the separation of differences in molecular size in solution. 
It is the method of choice for determining molecular weight distribution of synthetic polymers. In order to determine the amount of sample emerging, a concentration detector is located at the end of the column [76]. Additionally, detectors may be used to continuously determine the molecular weight of species eluting from the column. The volume of solvent flow is also monitored to provide a means of characterizing the molecular size of the eluting species. Other separation techniques such as high performance liquid chromatography (HPLC) can be used if needed [76].

\subsubsection{Scattering techniques}

\subsubsection{Laser light scattering}

Laser light scattering technique is a versatile characterization method for weight average molecular weights and radius of gyration of macromolecules [73, 74] It can also be applied to determine size and molecular weights of micelles in solutions. The Rayleigh ratio $\left(R_{\theta}\right)$, the angular variation of the excess absolute scattering intensity, is a function of the particle size and concentration and the wavelength of the incident radiation. Block copolymer micelles behave as colloidal particles, with dimensions 10-100 nm, where intra-particle interference becomes important, when the particles are no longer small compared to the wavelength of light, $(\mathrm{d}<\lambda / 20)$, in light scattering experiments. Further, classical light scattering is used to study the self-assembly structures of amphiphilic block copolymers due to its sensitivity to intermolecular interactions. Typically the relative scattered intensity versus scattering vector relationship, $R_{\theta}$ vs q, is fit via regression techniques to extrapolate dimensions of the particles. Common variations of these techniques include $\operatorname{Zimm}\left(R_{\theta}{ }^{-1} \mathrm{vs} q^{2}\right)$ and Debye $\left(R_{\theta}\right.$ vs $\left.q^{2}\right)$ and Berry $\left(R_{\theta}^{-1 / 2}\right.$ vs $\left.q^{2}\right)$ analyses [77]. 
Quasi-elastic light scattering (QELS) or photon correlation spectroscopy relies on the phenomenon that the rapid fluctuations in the re-radiated light are related to the diffusion rate of the scattering particles [78]. In dynamic light scattering, the time dependence of the light scattered from a very small region of solution, over a time range from tenths of a microsecond to milliseconds is measured. Then, these fluctuations in the intensity of the scattered light are related to the rate of diffusion of molecules in and out of the region being studied (Brownian motion), and the data can be analyzed to directly give the diffusion coefficients of the particles doing the scattering. When multiple species are present, a distribution of diffusion coefficients is seen. The diffusion rate is used to directly calculate the effective, geometry independent, hydrodynamic radius of the particles[79]. QELS or dynamic light scattering can characterize size distributions of dispersed particles in dilute solutions in the size range of $4-2500 \mathrm{~nm}$.

\subsubsection{Small angle $X$-ray and Neutron scattering}

Small-angle scattering (SAS) is a powerful technique for investigating structures of length scale with nanometer length scale $(1-300 \mathrm{~nm})$ [80]. The most important feature of the SAS method is its potential for analyzing the inner structure of disordered systems, and frequently the application of this method is a unique way to obtain direct structural information on systems with random arrangement of density inhomogeneities on such large scales. It is commonly used to investigate systems of particles in solution (micelles or colloids) and periodic systems (micelle networks or lamella). Small angle scattering data is the result of inhomogeneities in the scattering length density of a material and there in no need for crystallinity of the material [81]. 
In small angle X-ray scattering (SAXS), the scattering length density is a function of electron density. The x-rays interact with the electrons in the sample and the technique is therefore sensitive to electron density differences [82]. There exist both laboratory instruments based on more conventional sources and synchrotron based instrumentation for these x-ray measurements. In small angle neutron scattering (SANS), the neutrons interact with the nuclei in the sample and the interaction depends on the actual isotope [83]. Hydrogen and deuterium have very different scattering lengths and this can be exploited in contrast variation measurements in which it is usual that part of the molecule in solution is deuterated. It is also usual to change the scattering length density of the solvent by mixing deuterated and protonated solvents. The technique requires a neutron source, i.e. a nuclear reactor or an accelerator based spallation source and therefore the experiments are performed at large scale facilities.

The development and construction of third generation synchrotron radiation and high flux spallation neutron sources, have increased the potency of the technique for structural analysis of materials $[81,82]$ As far as data analysis is concerned, many methods have been developed for block copolymer micelle systems [83, 84]. Guinier and Fournet first collected the fundamental principles of small angle scattering in a classic monograph [82]. The methods mainly consist of non-linear least square fitting of analytical models to the small angle scattering data to describe intra-micellar scattering (the form factor) and the intermicellar scattering (the structure factor).

The implementation of these models requires careful treatment of the data and use of complementary techniques, such as cryo-TEM and NMR to extract physically meaningful data [16]. X-ray and neutron scattering techniques are complementary to each other, even 
though the basic theory is the same [85]. They offer a number of practical differences that can be exploited experimentally. For example, there is very little contrast in electron scattering length density between organic polymers and water matrix, while the nuclear scattering length density contrast can be varied by adjusting the $\mathrm{D}_{2} \mathrm{O} / \mathrm{H}_{2} \mathrm{O}$ ratio in SANS experiments.

\subsubsection{Cryogenic transmission electron microscopy (TEM)}

Cryogenic transmission electron microscopy (Cryo-TEM) has become an indispensable tool to obtain high-resolution direct images of complex liquids, namely liquids with structure on the order of nanometers to micrometers. The methodology that has been developed over the years helps us to capture the nanostructure in its native state of fixed concentration and temperature. A wide range of systems of low- and high-molecular weight solutes, synthetic and biological has been studied already by the technique. One of the widely studied system is the direct visualization of micellar structures formed by numerous block copolymers [86].

The samples can be prepared from rapidly vitrifying thin films of aqueous copolymer solutions to preserve the self assembled structures present at ambient temperature [87]. Different morphologies such as spherical, cylindrical and bilayer or vesical micelle structures have been visualized with cryo-TEM. Jain and Bates utilized cryo-TEM to observe 'Yjunctions' of cylindrical micelles formed from diblock copolymers. The 'Y-junction' structure had been predicted theoretically but had not previously been observed experimentally [88].

Several block copolymer micelles have been observed by cryo-TEM. Zheng and coworkers used cryo-TEM image analysis to determine the corona and core dimensions of 
poly(ethylene oxide)-b-poly(butadiene) micelles[10]. Lam and co-workers used imaging analysis techniques to average the dimensions from large numbers of spherical micelles formed from dilute solutions of Pluronic ${ }^{\circledR}$ F127 [89]. PEO-based block copolymers were studied by cryo-TEM imaging by Won et al [90]. The series of diblock copolymers investigated existed in a number of micellar morphologies including vesicle bilayers, cylinders and spheres.

\subsubsection{Polymer phase properties}

Differential scanning calorimeter (DSC), tube inversion technique and simple potentiometric titrations have been utilized to study the phase properties of copolymers. Calorimetric techniques have been utilized to investigate the origins of micelle and gel formation of amphiphilic copolymers. The simplest of measurements, and probably the most widely used, is the tube inversion test to find the gelation temperature. Most researchers use similar criteria for the differences between solution and gel. Upon inversion, the contents of a gel must remain set for a few minutes to be considered a gel.

The onset of micelle formation in water is usually accompanied by an endothermic first order transition, corresponding to the energy of dehydration of the hydrophobic block [80]. This is in agreement with the evidence that micellization is driven by entropic gains of segregating the hydrophobic block segments to a core domain. Previous studies on copolymers such as Pluronic ${ }^{\circledR}$ copolymer micelle self-assembly established an empirical relationship between copolymer concentration, hydrophilic/hydrophobic composition and the critical micellization temperature (CMT) $[91,92]$. While the formation of Pluronic ${ }^{\circledR}$ micelles results in an easily distinguished transition, the onset of macroscopic gelation appears to have only a minor energy of transition. Hence a simple tube inversion technique is employed to 
study the macroscopic gelation as a function of temperature. Potentiometric titrations will be utilized to find the $\mathrm{pKa}, \mathrm{pI}$ values of the polymers in aqueous solutions, thereby to predicting its ionization behavior with $\mathrm{pH}$ change [93].

\subsubsection{Mechanical properties}

The mechanical properties of physical hydrogels formed from block copolymers have been investigated with a number a techniques. Additionally, rheology and mechanical studies offer an excellent means of investigating the sol-gel transition temperature of thermoreversible hydrogels [94] The viscoelastic properties of hydrogels are highly dependent on temperature, concentration and chemical environment. The drug delivery applications of hydrogels are determined largely by the viscoelastic properties of the gel in vivo. The value of the shear modulus of a hydrogel is indicative of the structuring in the system. Investigations of the rheological properties of Pluronic ${ }^{\circledR}$ copolymers $[95,96]$ PLLAPEG-PLLA/PDLA-PEG-PDLA polymers [97], Pluronic ${ }^{\circledR}$-g-PAA [98] and PEG-g-PLGA $[68,97]$ have been conducted.

Rheological instrumentation is used for various reasons, for example, to determine yield stress (stress that must be exceeded for flow to occur) to help predict shelf-life and strain sweeps to determine the critical strain (minimum energy needed to disrupt structure, where the higher the critical strain the better the systems is dispersed). Wang and co-workers used rheology to determine the CMT and the gelation point of Pluronic ${ }^{\circledR}$ solutions [95]. The gelation point was marked by a $10^{3}$ fold increase in the shear modulus within a small temperature range. Upon gelation the shear modulus did not significantly increase with temperature. Wang and Johnston employed a pulse shear meter to measure the shear modulus of solutions of Pluronic ${ }^{\circledR}$ formed hydrogels [99]. This method is especially useful for 
hydrogels because it is very non-invasive, thus minimizing disruptions to the gel formation process. The heat transfer through the polymer solutions dominated the kinetics of the gelation process. This result has significant implications for the potential injection of Pluronic ${ }^{\circledR}$ based drug delivery devices. A slow-forming gel, once injected, could result in a 'burst effect,' as a low viscosity gel network does not posses the zero order release properties that make Pluronic ${ }^{\circledR}$ hydrogels an attractive delivery device [100].

\subsection{Applications of block copolymers in biomineralization}

\subsubsection{Naturally occurring hierarchical structures}

Scientists and engineers have long been inspired by the intractably complicated structures and functional properties of the materials formed in nature. Intriguing selfassembled hierarchically ordered self-assembled biominerals are commonly observed in nature. Biominerals are complex in structure and function, and are difficult to mimic through synthetic processes $[101,102]$. The minerals are typically biocomposites as they are typically close in association with organic polymeric phase. Biominerals include, hard tissues of organisms, for example, ivory, coral, oyster shells, sea urchin spines, cuttlefish bone, skeletal units of single-celled organisms, magnetic crystals in magnetotactic bacteria, limpet teeth and human bones[1, 103] and highly ordered inorganic-organic soft tissue nanocomposites, such as membranes, skin, spider's silk, muscles and cuticles [104].. Biominerals are formed at physiological aqueous environments, under mild conditions using biomacromolecules as templates, and normally exhibit structures and shapes that are very different from the crystals produced inorganically $[103,105]$. Unlike synthetic inorganic crystals, these biomineral crystals may not have defined crystal properties but may be molded into elaborate structures which have non-faceted crystal surfaces. The ability to grow and stabilize such energetically 
unfavorable crystalline structures within the physiological environment has intrigued the scientific world. Nature has taught that the control over biomineral properties can be accomplished at different hierarchical levels, including the regulation of particle size, shape, crystal orientation, polymorphic structure, defect texture, and particle assembly which results in structures with hierarchical organization optimized in different length scales [106, 107].. Further it is believed that the biological molecules in such a way that hierarchical composite structures can be built with numerous functionalities with excellent physical and chemical properties.[108-111]. The low temperature nanomaterials synthesis using a biomimetic bottom-up approach is versatile in future biomaterials design and applications at physiological temperatures.

\subsubsection{Natural mineral formation process- Bone as an example}

Human compact bone is a natural nanocomposite which exhibits a rich hierarchical structure $[70,112,113]$ with excellent mechanical properties and biocompatibility. On the microstructural level are the osteons [104, 112], which are large (200 micron dia.) hollow fibers composed of concentric lamellae and of pores.
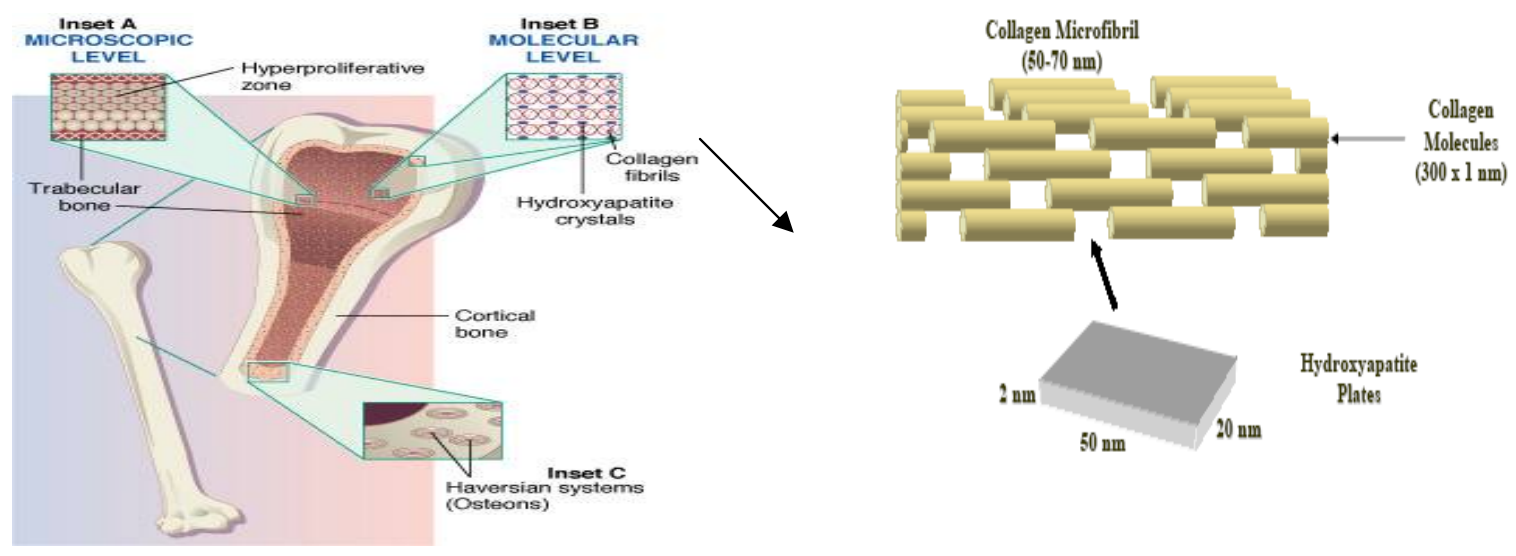

Figure 2.12. Microscopic and molecular level structures of bone and illustration (adapted from [113]) 
The lamellae are built of fibers, and the fibers contain fibrils. At the ultrastructural level (nanoscale) the fibers are a composite of the mineral hydroxyapatite and the protein collagen. The natural bone is a composite of collagen, which is a protein based-hydrogel template with carbonated apatite crystals with different compositions and microstructures [104]. The growth of the calcium phosphate is facilitated by the structural frame work of collagen matrix. The unique mechanical properties of such a low stiffness, resistance to tensile and compressive forces and high fracture toughness of the bone is believed to be due to the unusual combination of a hard inorganic material and an underlying elastic protein hydrogel [114].

The pore structure of bone is essential in maintaining its viability and consequently its ability to adapt to mechanical stress. A two level hierarchical analytical model $[104,112$, 114] has been used to predict anisotropic elasticity of bone; it successfully modeled how bone stiffness depends on the orientation of applied stress with respect to the osteon axis. The triple helical collagen macromolecule is formed as a result of the amino acid glycine occupying every third unit. The strongest intermolecular attractions occur when neighboring molecules are shifted by $67 \mathrm{~nm}$, the 'stagger' which is responsible for the banded appearance of collagen observed via electron microscopy. Assembly of subfibrils into fibrils is thought to be controlled at least in part by the primary structure of collagen. The larger scale organization is attributed to interaction with non-collagenous components such as proteoglycan matrix. The fibers are not perfectly aligned; they form a wavy or crimp structure which confers upon the tendon an initial compliance as the fibers straighten under load. Moreover, the damage processes which govern the strength and toughness of tendon involve structural elements over the full hierarchical range of sizes. 


\subsubsection{Biomimetic mineralization for hierarchically ordered materials design}

The spontaneous organization of simple molecules into well defined nanostructures is of fundamental importance and has many applications in biotechnology and materials sciences [115]. The traditional top-down lithographic techniques limit the synthesis of complex architectures, where nanomaterials $<100 \mathrm{~nm}$ are not easily attainable at present by lithographic techniques, but can be realized with biological self-assemblies such as the tobacco mosaic virus, collagen, capsid [116], tubulin[117], or actin[118, 119].

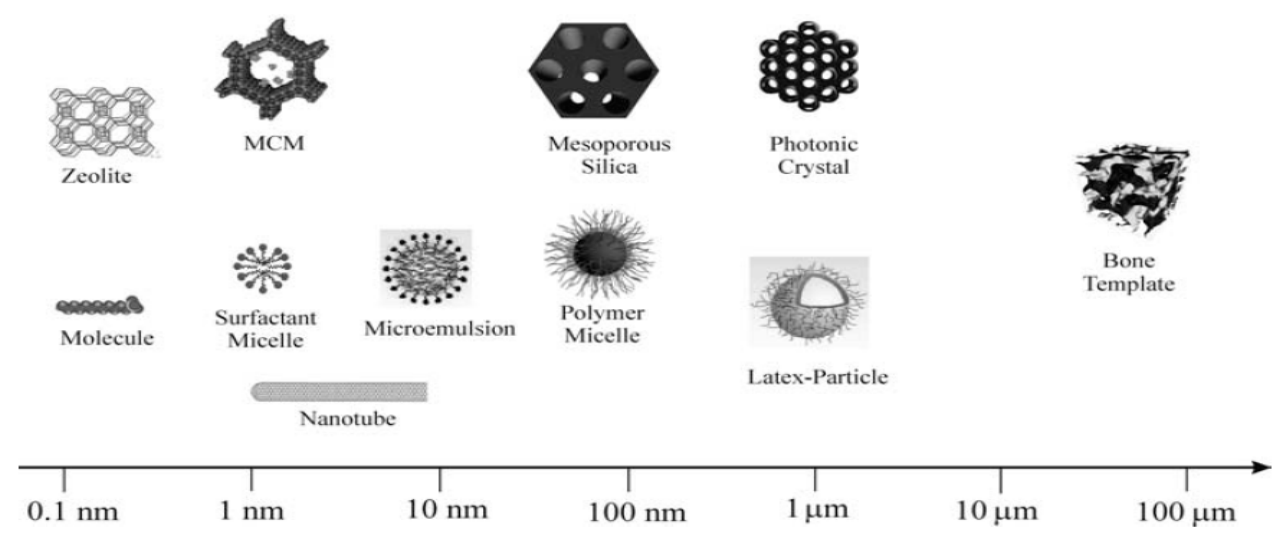

Figure 2.13. Complex functional structures can be obtained by combining bottom-up and top-down approaches (From [1])

These biomolecules spontaneously organize into well-defined nanostructures with nanometer size dimensions. This inspiration from natural fibers is difficult to implement when the building blocks themselves are complex, as in the case of proteins and of their high fabrication costs. Several alternative routes have emerged based on molecules that selforganize in a programmed way [120-125]. The design of such biomimetic systems requires the understanding of the relationship between the molecular structure and the self-assembly process of the nanostructures. 
In a fundamental or classical crystallization process, the process starts from nucleation clusters, usually consisting of ions involved in the formation of inorganic minerals, from which the primary nanoparticles are nucleated leading to the formation of the smallest crystalline units capable of further growth [102]. These crystalline units grow further by ion attachment and unit cell replication, resulting in a final macroscopic single crystal. However, the complex crystal structures formed in nature are often called as nonclassical crystallization, where they are developed through more complicated mesoscale transformations [71] involving organic templates. In these processes, nanocrystals are also formed, but due to face-specific information of the template surfaces they self-assemble in a controlled way. In the simplest case, oriented attachment could occur leading to single crystal, due to the different surface energies of the surfaces and consequent decrease of high energy surfaces by crystallographic fusion. However, nature made it even complex by encoding the surfaces with a variety of protein molecules [111, 112]. Face specific interactions between nanocrystalline building units is believed to lead to directed selfassembly, resulting in a so called mesocrystal. These developing new concepts are essential to understanding the biomineralization process in detail and hence to self-assemble materials from crystalline nanoparticles through a bottom-up approach.

\subsubsection{Hierarchical organization of block copolymers}

By integrating environmentally sensitive polymer blocks into amphiphilic block copolymers, self assembled supramolecular structures that exhibit stimuli or environmentally responsive properties can be obtained $[1,2]$. Self-assembly is a powerful tool in assembling and forming structures with nanoscale dimensions. It refers to reversible processes which can be controlled by proper design and conditions of initially ordered components [3]. Figure 
2.14 illustrates the dimensional evolution of self assembled functional materials. Supramolecular structures containing environmentally sensitive bioinspired smart copolymers are formed by this process.

Polymer chemistry has made tremendous progress in controlling the primary structure of homo- and heteropolymers as well as the recurring order in the bulk by phase separation [126]. Block copolymer are ideal candidates for developing novel nano architectures due to their great synthetic accessibility over large dimensions and tunable aspect ratios which cannot be achieved by hetero polymers.

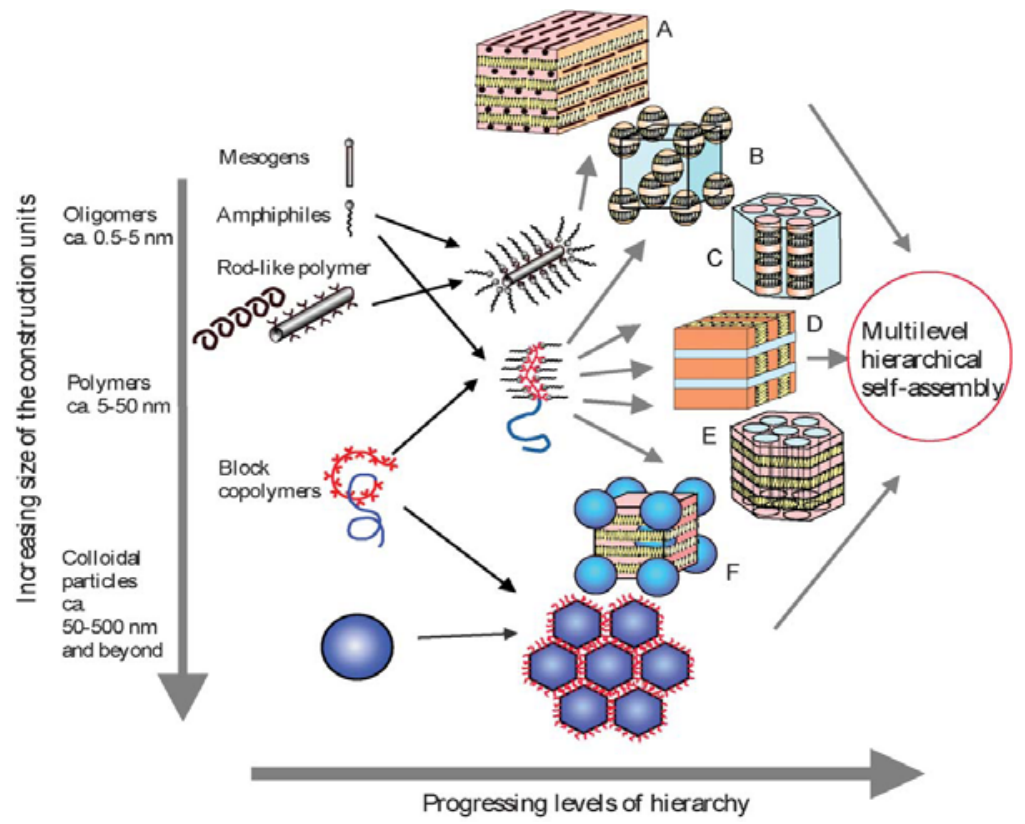

Figure 2.14. Potential scenarios to construct hierarchically self-assembled polymericstructures. Construction units of different sizes allow a natural selection of different selfassembled length scales (From [3] ).

The block copolymer self-assembly into different structures can be divided into dilute solutions (micelles, cylindrical micelles, and vesicles), lyotropic phases of higher concentration, and bulk phases, where each of them contributes for the preparation of various interesting materials [127]. Micelle formation is a simple and well-known example of the 
self-assembly of polymers. The block copolymers self-assemble to a micelle with defined size and shape. The size of the micelle depends on the length of the polymer blocks [128]. Block copolymers with large soluble blocks form spherical micelles preferentially. Figure 2.15a [129], shows the spherical micelles of PS-PI system, whereas cylindrical micelles or vesicles result from smaller soluble blocks. Cylindrical micelles, for example, of poly(butadiene-b-ethyleneoxide (PB-PEO); Figure 2.15b [130] may have lengths of several micrometers. Block copolymer vesicles can be formed over several length scales with diameters from $100 \mathrm{~nm}$ up to several micrometers. Further, polymersomes are mechanically and thermodynamically much more stable than the well investigated lipid vesicles, and hence good for the encapsulation and the release of substances. Vesicles of poly(2-vinylpyridine-bethylene oxide) (P2VPPEO) of length scales around $10 \mathrm{~mm}$ (giant vesicles) form, (Figure 2.15 c) [131] into which, for example, fluorescent dyes can be encapsulated.
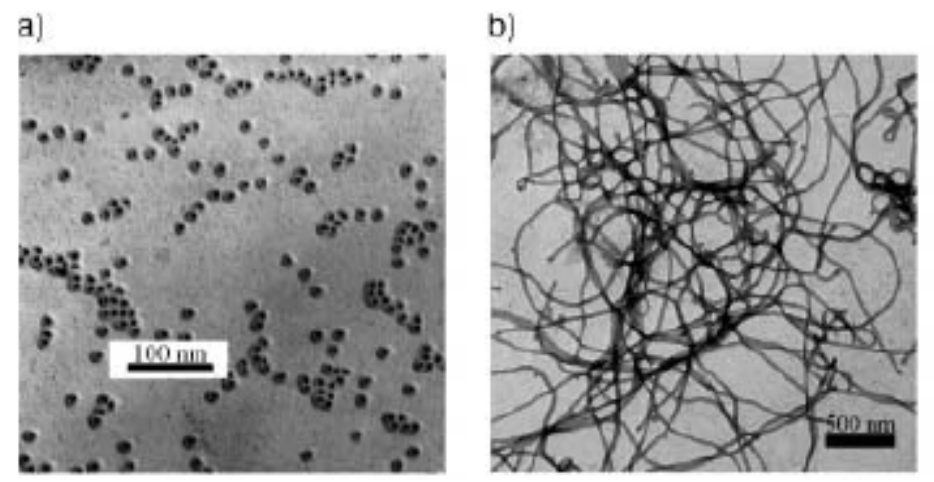

c)

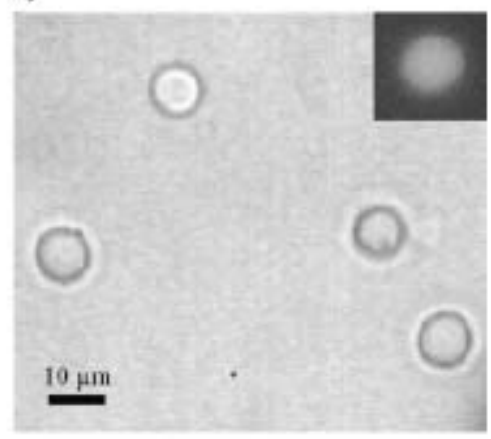

Figure 2.15. Electron micrographs of (a) spherical micelles (PS-PI/DMF), [129] (b) cylindrical micelles (PB-PEO/water), [130] (c) vesicles (P2VP-PEO/water) [131]

Lyotropic liquid crystalline phases are formed with higher concentrations of the block copolymers [132]. The stability of these structures can strongly depend on temperature. In aqueous solutions poly-(ethylene oxide) (PEO) is usually the soluble block, but increase in temperature reduces the solubility of the PEO block, resulting in phase transitions. The 
lyotropic phase behavior of block copolymers of poly(ethylene oxide-b-propylene oxide-bethylene oxide) (PEO-PPO-PEO), Pluronic ${ }^{\circledR}$ was well studied [132]. Lyotropic phases can be applied to the preparation of porous materials while cubic micellar phases are also used in gel electrophoresis to separate proteins and oligonucleotides. In a similar study with pluronic based pentablock copolymers studied by Determan et al., it has been shown that the pentablocks self assemble and form various morphologies [120, 121].
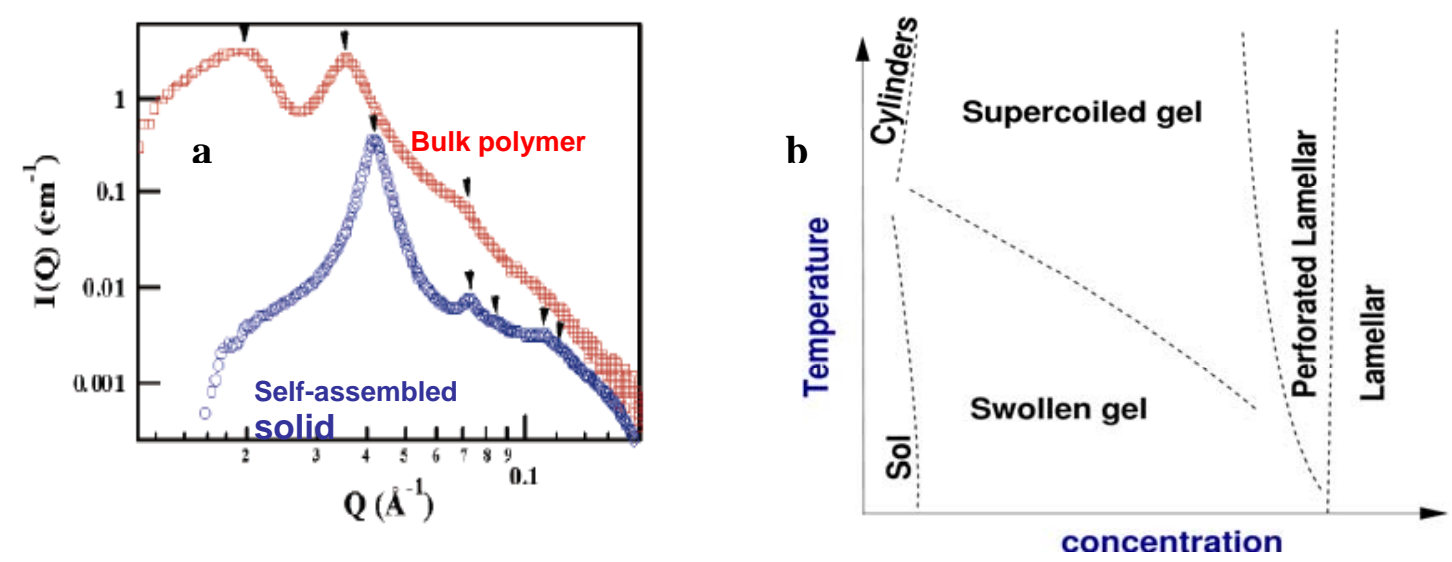

Figure 2.16. a) Self-assembly of PDEAEM pentablock copolymer [133] b) phase diagram of the pentablock copolymer self-assembly from molecular simulation [134].

Block copolymers in a micro-phase-separated state are also present in bulk. A variety of copolymer morphologies such as lamellae (LAM), hexagonally ordered cylinders (HEX), arrays of spherical microdomains (BCC, FCC), modulated (MLAM) and perforated layers (PLAM), ordered bicontinuous structures such as the gyroid, as well as the related inverse structures can be obtained [1]. Morphology mainly depends on the relative block length. For example, both blocks are of identical length, lamellar structures are formed. The stability ranges of the different structures can be represented in a phase diagram as shown in Figure 2.17 for the system poly-(styrene-b-isoprene) (PS-PI) [135]. The periodic length scale of the super lattice can be adjusted over the whole mesoscopic range (from a few up to several 
hundred nanometers) by varying the degree of polymerization. Particulate structures such as spherical and cylindrical micelles as well as vesicles form in dilute solution. Spherical micelles with cubic packing (FCC, BCC), hexagonally packed cylindrical micelles (HEX), and lamellar phases (LAM) form can result in the formation of complicated super lattices.

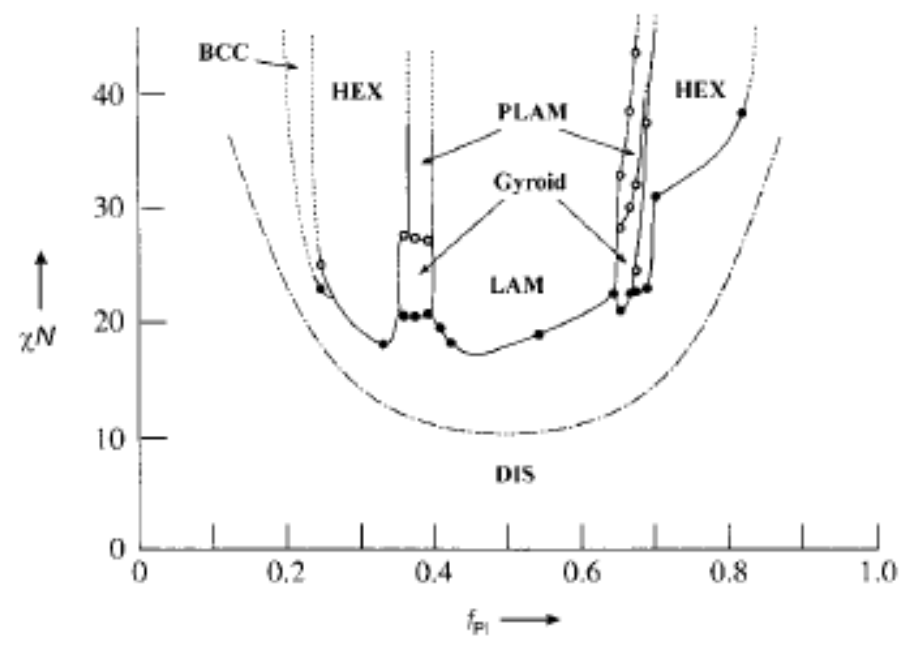

Figure 2.17. Experimentally determined phase diagram of PS-PI [135]

From natural structures, it has been seen that several hierarchies of self-organization are often needed to realize a functional system [136, 137]. Collagen, a connective tissue protein, which consists of helices which form triple helix structures with excellent mechanical properties is an example. As mentioned earlier shown in Figure 1, several hierarchy levels can also be distinguished for block copolymers, their characteristic lengths ranging from $1 \mathrm{~nm}$ up to $10 \mu \mathrm{m}$ [3]. The different self-organizing structures of surfactants and block copolymers are shown in Figure 2.18 [1].

Molecular self-assembly is a powerful tool in synthesizing architectures with complementary regions that recognize each other, interlock, and automatically or by means of external assistance, organize into larger entities held together by non-covalent interactions. 
Although nature has many biologically complex structures, the biological evolution is limited by specific environmental situations, and the structural advances are selected in the need of an immediate evolutionary benefit. On the other hand, synthetic organic and supramolecular chemistry can rely on a much wider pool of organic interactions, program lock-and key situations, and thus could potentially design structures with novel properties. High degree of structure control can be achieved by block copolymers by proper design of the block copolymer [138]. The block lengths can be successfully controlled by living polymerization and hence the aggregation number and thus the size of the micelle or the long range order can be controlled [139, 140]. Further depending on the volume fraction of the block copolymer there is formation of well-defined superlattices in lyotropic phases or in the solid phase.

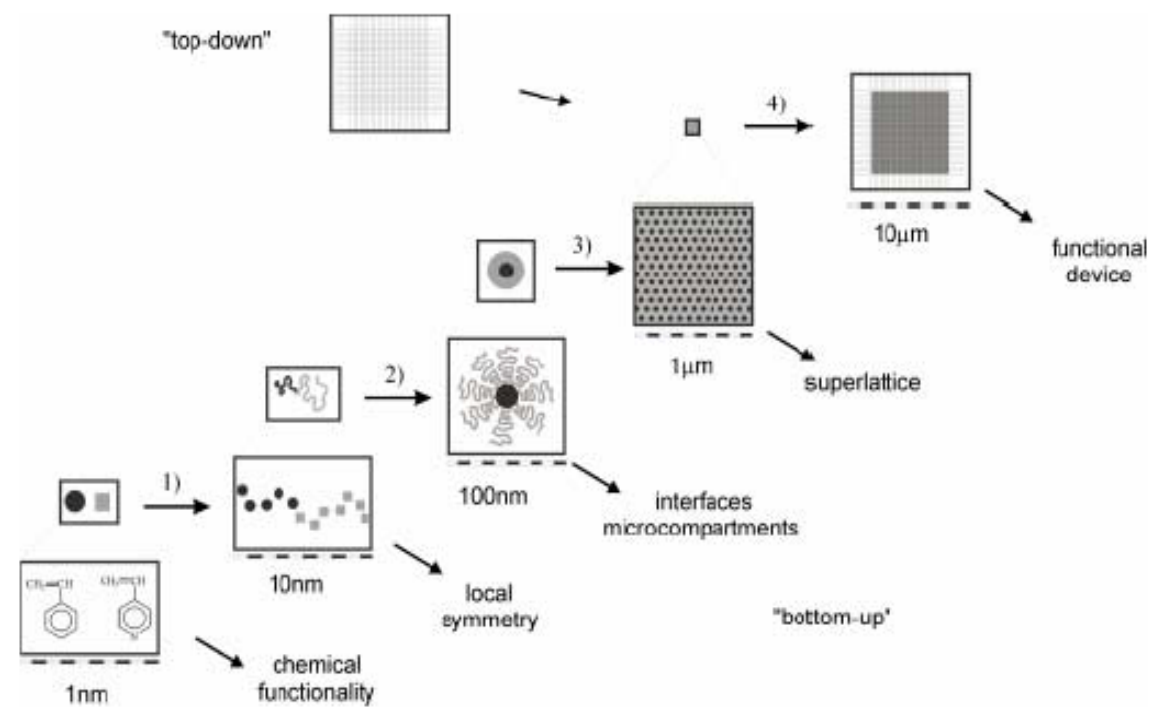

Figure 2.18. Various structure hierarchies in block copolymer systems [1]

\subsubsection{Biomimetic mineralization using polymers as templates}

Many explorations are underway to find novel bio-inspired strategies to create organized nanostructures spanning several length scales with the same kind of specialization and complexity that exists in nature $[1,141]$. Biominerals in nature are usually produced in 
the presence of an organic template that controls the inorganic crystallization, growth and self assembly. By utilizing a suitable nanoparticle self-assembly process these materials can be recreated successfully resulting in organic-inorganic hybrid structures with complex form and hierarchical structural order. Various techniques are currently been researched on biomimentic synthesis such as face-selective coding of nanoparticle surfaces by additives for controlled self-assembly, producing single crystals and nanoparticle arrays by mesoscale self-assembly, balancing aggregation and crystallization and transforming and reorganizing of pre-formed nanoparticle building blocks [142]. Recent advances in the field show the feasibility of designed artificial organic templates for mimicking natural mineralization processes [143]. Figure 2.19 shows the natural templating process of bone formation.

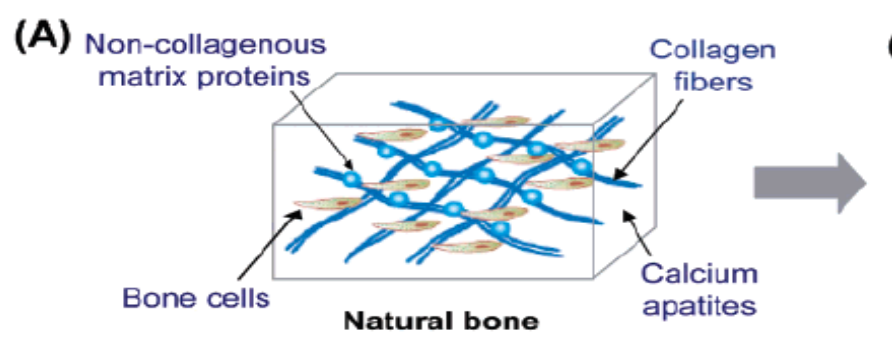

(B)

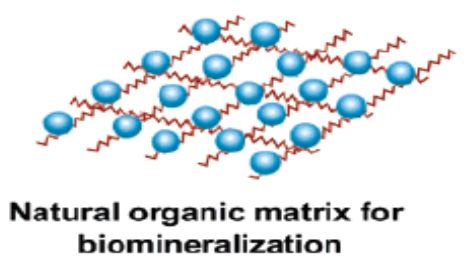

Figure 2.19. Recreate natural bone synthesis with synthetic polymers functionalized to mimic the mineral-nucleating protein in bone [143]

Here a functional polymer template can be directly used for the mineralization synthesis of novel inorganic nano architectures. Further water-soluble polymers are used as crystal modifiers for controlled crystallization in designing inorganic materials [144]. The use of a soluble polymer matrix is intriguing since they are responsible for the crystal morphogenesis and modification in different unexplored ways whereas the insoluble 
inorganic matrix defines the crystallization compartment. Although several attempts are currently underway in recreating natural structures, the perfection of mimicking the natural synthesis is still not possible to attain.

Most of the self-assembling processes known in biomineralization concern organic molecules which are often biopolymers that form the structural or insoluble matrix. The role of the organic scaffold is either to act as a scaffold or template for the mineralization process as collagen does in bones, amelogenin in tooth enamel, and surface layer proteins in bacterial cell walls or confines the mineralization space such as in vesicles like cocoliths, diatoms and magnetotactic bacteria. Apart from the insoluble organic matrix, there is also soluble organic matrix. The task of this soluble organic matrix is to control nucleation, growth, orientation, and polymorphs of the inorganic compounds. This is further proven by the example, where extraction of soluble protein from the aragonite layer of nacre and then applying them for subsequent $\mathrm{CaCO}_{3}$ crystallization resulted in aragonite precipitation rather than the calcite precipitation [107, 145]. The function of the insoluble organic matrix is difficult to understand, as it usually consists of many biopolymers in small quantities. Thus an artificial organic matrix consisting both soluble and insoluble molecules could be an efficient way to understand the function of the scaffold as well to replicate the process.

\subsubsection{Block copolymers and other polymeric materials in biomineralization}

Several block copolymers were used in biomineralization for nanoparticle synthesis. Double hydrophilic block copolymers (DHBC) were used in biomimetic materials design. The polymers are mostly bioinspired polymers where one polymer interacts with the inorganic and the other help to promote solubilization, similar to protein interaction with mineral (acidic and basic units of amino acids) [146]. Solvating block is mostly PEO block 
and the binding block contains various chemical pattererns showing affinity to mineral. Several nanocrystals Pd, Pt [147-149], Au [147-150], Ag [151], lanthanum hydroxide [152] and several inorganic particles, $\mathrm{CaCO}_{3}[144,153-158], \mathrm{BaCO} 3, \mathrm{PbCO} 3, \mathrm{MnCO} 3$ [143], chiral organic crystals [159] were synthesized for DHBC polymeric templates. Recently Drew and coworkers have demonstrated the ability of Pluronic based pentablock copolymer self-assembling nanostructures as potential templates for inorganic nanocomposites synthesis [160].

Synthesis of mesocrystals is also followed by a biomimetic approach. Hexaganol prismatic seed crystal of fluoroapatite is a most investigated mesocrystal formed in a gelatin gel and further grows to spherical particles through dumbbell intermediates [161, 162]. The internal structure of the hexagonal seeds revealed a radical pattern with a superstructure periodicity of $10 \mathrm{~nm}$. Although it has been suggested that the dipole moment be perfect for their perfect alignment, the process of self-assembly from the nanoparticle building units is still under study. Other studies of biopolymeric matrix crystal growth include agar, gelatin and pectin gels where triclinic crystals $\left(\mathrm{H}_{3} \mathrm{BO}_{3}\right.$ and $\left.\mathrm{K}_{2} \mathrm{Cr}_{2} \mathrm{O}_{7}\right)$ were formed in curved and helical branch shapes [163, 164], Calcite structure with high quality and periodic order was synthesized using poly(acrylic acid) and a substrate of cholesterol bearing pullulans [165]. Copper oxalates are another example of mesocrystals where the nanoparticles were arranged in terms of morphology of hydroxyl-methylpropylcellulose (HPMC) polymer [166]. The nucleation, nanocrystal growth and aggregation by face selective interaction are influenced by the polymer.

By using surfactant-mediated nanoparticle self-assembly $\mathrm{BaCrO}_{4}$ and $\mathrm{BaSO}_{4}$ nanoparticles were synthesized in reversed micelles, where a surfactant coated self- 
assembling structures were obtained [167]. Both nanoparticle crystallization and surfactant interactions led to self-assembly of one or two dimensional structures. Inverse micelles for water induced mesoscale transformation of amorphous calcium carbonate nanoparticles. In another approach, a polymer controlled crystallization method was used where the structure is formed in synergistic effect of polymer and inorganic interaction, and a subsequent formation of the crystal structure is obtained. Unusual superstructures of helical $\mathrm{CaCO}_{3}$, complex spherical $\mathrm{BaCO}_{3}$, hollow octacalcium phosphate and $\mathrm{BaCrO}_{4}$ fiber bundles were formed by using polyelectrolyes such as polyacrylic acid or polyaspartic acid as crystal growth modifiers $[159,168,169]$. Other polyelectrolytes used in biomimetic synthesis include, polyacrylamid and carboxyl-functionalized polyacrylamides in producing hexoganal ringlike $\mathrm{ZnO}$ structures [170] and a poly(allylamine hydrochloride)-poly(sodium 4styrenesulfonate) for the hydroxyapatite composite shells formation [171].

Dendrimers, foldamers, graft polymers and supramolecular functional polymers are other polymeric materials that are widely used in biomimetic mineral synthesis. Dendrimers were widely used in the synthesis of $\mathrm{CaCO} 3$ crystals $[172,173]$. It has been shown that vaterite particles with particles sizes in the range of 2.3-5.5 nm were obtained using dentrimeric templates [173]. Foldamers were used in the synthesis of calcite crystals, where elongated calcite crystals with newly expressed crystal faces were formed [174]. Recently poly(ethylene oxide) modified graft polymers were used in the crystals synthesis of $\mathrm{ZnO}$ and $\mathrm{CaCO} 3[175,176]$.

\subsubsection{Biopolymers in biomineralization}

Template-controlled crystallization is a recent method in biomineralization. The aim

of this templating is to develop a synthetic approach to mimic a specific part of a 
biomineralization process, starting from the molecular level. In this respect, self-assembled peptide amphiphiles combined with hydroxyapatite crystallization to mimic bone, copolypeptides to mimic properties of silicatein in $\mathrm{SiO}_{2}$ mineralization are important examples, where block copolypeptides of poly(L-cystine 30 -L-lysine 200$)$ were used [177]. Recently Deming and coworkers have reported the synthesis of hexagonal silical platelets using poly(lysine) polypeptide templates [44]. Biotemplates with well-defined chemical and structural properties have been recently used in synthesizing precise controlled size and shape material nanostructures. Nanostructures of $\mathrm{Ag}$, Ni and Co nanowires, $\mathrm{CdS}, \mathrm{PbS}$ and $\mathrm{ZnS}$ nanostructures are some of the structures that has been synthesized using these biomimetic approach $[178,179]$. Biopolymer directed mineralization were also used in the synthesis of $\mathrm{Au}$ and $\mathrm{ZnS}$, where organized nanostructures with different morphologies and dimensionalities were obtained with DNA [180-183], virus [184] and peptides [179]. Several mineral specific binding peptides were identified by phage display and other methods for biomineralization $[101,185,186]$. A collagen binding peptide, identified from osteopontin shown to induce bone mineralization [187]. Recently Cheng et al showed that an aspartic acid rich 14 aminoacid peptide sequence has the affinity to nucleate and enhance hydroxyapatite invitro [186]. Self-assembled bacterial superstructures can be used to template superstructures of magnetite, calcium sulfide or preformed silica particles by swelling in the colloidal sols. Similarly, sponge-like gels were used to infiltrate precursors of magnetite or titanium dioxide by rapidly loading the gel to perform mineralization $[188$, 189]. The structural replication was limited due to the limited loading of precursors. Colloidal crystals were also used as templates where gold or ceramic nanoparticles were infiltrated into colloidal crystals and replicas were obtained $[190,191]$. These examples show 
that soft organic templates could be used for templating inorganic structures and could be a potential method for future materials design.

\subsection{Biomedical applications of stimuli responsive polymers}

\subsubsection{Drug depot}

A successful drug carrier system needs to demonstrate optimal drug loading and release properties, long shelf-life and low toxicity. Colloidal systems, such as micellar solutions, vesicle and liquid crystal dispersions, as well as nanoparticle dispersions consisting of small particles of $10-400 \mathrm{~nm}$ diameter show great promise as carriers for drug delivery systems $[192,193]$. Development of efficient drug delivery systems with synthetic polymeric materials is currently the focus of intense academic and industrial research [192-194]. In particular, polymeric controlled drug release in response to environmental stimuli is an essential step towards the development of a self regulated drug delivery formulation [194, 195]. Smart block copolymers with amphiphilic character capable of forming micro- or nanosized micelles, have been shown to be effective for controlled drug delivery applications, due to the high loading capacity of the micelle structure [195].

Recently, thermo responsive hydrogels that are not chemically crosslinked, but are formed by entanglement of polymer micelles, have attracted much attention, as they are biodegradable, precluding invasive removal procedures of the polymeric material unlike chemically crosslinked hydrogels [153-156]. Thermoreversible polymers such as PPO and NiPAAm are widely used in these systems [196]. The unique nature of these materials are they are low viscosity liquids at low temperatures and can form a physically crosslinked gel phase around physiological temperatures. This will allow the drug loaded in liquid form followed by in situ hydrogel formation. In vivo, the ingress of water into the hydrogel results 
in dissolution or degradation of the hydrogel matrix, resulting in sustained release of drug. Hydrogels as drug delivery systems are very promising if combined with the technique of molecular imprinting [196]. They are used to regulate drug release in reservoir-based systems or as carriers in swelling-controlled release devices. On the forefront of controlled drug delivery, hydrogels, as environ-intelligent and stimuli-sensitive gel systems, can modulate drug release in response to $\mathrm{pH}$, temperature, ionic strength, electric field, or specific analyte concentration differences. Release can be designed to occur within specific areas of the body. Among the stimuli, $\mathrm{pH}$ is the most important one due to different physiological $\mathrm{pH}$ has been seen around the body [197]. Depending on the pKa values suitable polymer hydrogel could be chosen for $\mathrm{pH}$-stimulated release.

Several stimuli-responsive hydrogels are used as drug carriers. Hydrogels made with poly anions crosslinked with azoaromatric cross linkers are used in colon specific drug delivery (Figure 2.20) [198]. Other examples include boronic acid hydrogels to impart glucose sensitive insulin release, poly(amidoamine) based polymer hydrogels for endosomal drug delivery and poly (acrylic acid) based hydrogels for oral insulin delivery [198].

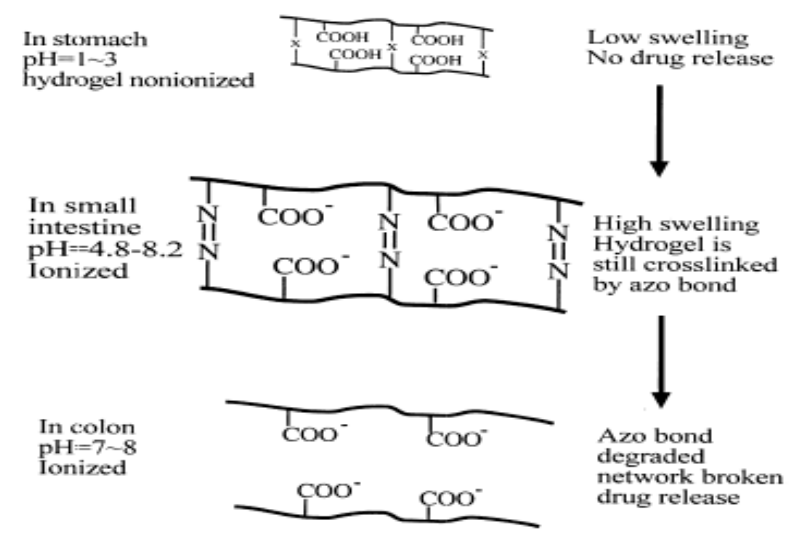


Figure 2.20. Schematic illustration of oral colon-specific drug delivery using biodegradable and $\mathrm{pH}$-sensitive hydrogels [198]

Other hydrogels including a glucose sensitive hydrogel based on novel phenyl boronic acid monomers has been developed. The LCST of N-Isoporopylacrylamide hydrogels incorporating $5-20 \%$ of the phenyl boronic acid monomer could be varied between 22 and $34^{\circ} \mathrm{C}$ by increasing the concentration of glucose in solution. This material has great potential as a self regulated, glucose-sensitive device that may aid in the treatment of diabetes $[35,199,200]$. Recently, biohybrid hydrogels that show environmentally responsive properties have been designed. Glucose sensitive gels have been prepared which function by entrapping glucose oxidase, an enzyme that reacts with glucose to form gluconic acid, within a polycationic hydrogel. When glucose concentrations increase in the presence of the hydrogel, the gluconic acid produced lowers the $\mathrm{pH}$ in the microenvironment of the hydrogel [201]. This in turn causes swelling of the hydrogel network and entrapped insulin is released. Another attractive example of a biohybrid hydrogel is a hydrogel with calcium binding protein that undergoes large conformational change upon binding to calcium ions thereby showing calcium ion stimuli responsiveness [202]. The detailed mechanism of this hydrogel swelling is shown in Figure 2.21. The potential application of this hydrogel could be as microactuators in responsive drug delivery systems [202].

The drug release is accomplished only when the polymer swells. One of the most remarkable, and useful, features of a polymer's swelling ability manifests itself when that swelling can be triggered by a change in the environment surrounding the delivery system. Depending upon the polymer, the environmental change can involve $\mathrm{pH}$, temperature, or ionic strength, and the system can either shrink or swell upon a change in any of these 
environmental factors. For most of these polymers, the structural changes are reversible and repeatable upon additional changes in the external environment.
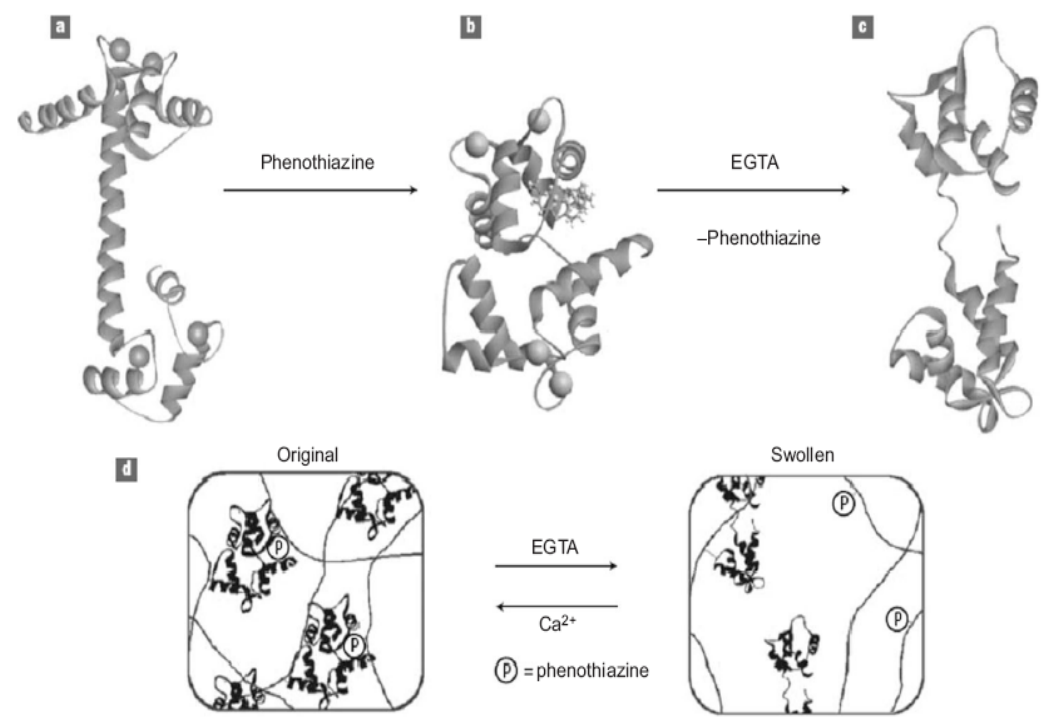

Figure 2.21. Genetically engineered biohybrid hydrogel and swelling of stimuli-responsive hydrogel. Calmodulin (CaM) can have three different conformations a) dumbbell (spheres represent four bound $\mathrm{Ca}^{2+}$ ions and are larger for emphasis) b) CaM with bound phenothiazinebound ( $\mathrm{Ca}^{2+}$ and ball-and-stick structure for phenothiazine shown) c) native confirmation in the absence of $\mathrm{Ca}^{2+} \mathrm{d}$ ) Hydrogel swelling mechanism in response to ethylene glycol tetraacetic acid (EGTA) [202].

\subsubsection{Injectable photocrosslinkable hydrogels for post traumatic arthritis cartilage}

\section{tissue healing}

Post traumatic arthritis develop after a fracture of torn ligament, due to the ligaments or supporting materials become unstable at the joint of injury [203, 204]. As a result increased stress at the joint cartilage leads to traumatic arthritis or osteoarthritis. Hence its important to have a supporting material during the fracture healing process. The supporting material should be structurally resemble the cartilage tissue, should be biocompatible, 
integrate into the surrounding tissues and the ability to adopt according to physiological requirements.

Injectable hydrogels have attracted scientists due to their minimally invasive nature in various biomedical applications [51, 205]. Among which tissue engineering applications with photocross linkable gels are currently under investigation. In situ thermoresponsive hydrogels with additional covalent crosslinking through photopolymerization will have improved mechanical properties and are ideal for tissue engineering applications. Hubbell and coworkers introduced in situ photopolymerizable hydrogel networks, in which poly(ethylene glycol) (PEG) was used as central block, with side blocks with either acrylates or oligo ( $\alpha$-hydroxy acids) [50], Figure 2.8.2.1 represents some of the in situ photocrosslinkable polymer structures.

Several photocrosslinking hydrogel structures are under investigation for tissue engineering applications. The crosslinking hydrogel networks are created by insitu polymerization using UV light and photoinitiator to create free radical to initiate polymerization and hence the crosslinked structures [206]. Recently, the advances in photopolymerizable hydrogels for tissue engineering applications were summarized by Nguyen and West [55]. More recently, photopolymerizable hydrogels including hyperbranched methacrylated polyglycerol [207], phosphoester-based polymers [208, 209] and lactic and caproic acid segments containing copolymer [210] were reported for the use in tissue engineering applications. PFF (phenol-furfural) which is known to its biocompatibility and biodegradability could be photocrosslinked with different photoinitiator systems [211, 212]. 
The major design considerations in photocrosslinking are the UV wavelength, intensity, photoinitiator, and amount of crosslinking groups. UV lights of various wavelengths have been tested for biocompatibility and for its potential in polymerizing and crosslinking chemical structures. Further, the concentration of photoinitiator, the light intensity and the reactive double bonds in the formulations were optimized to have effective

(A)

(C)

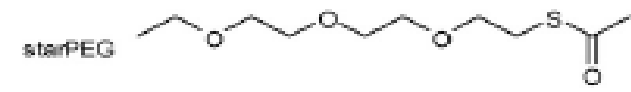

(D)<smiles>CCC(C)(CC(C)(C)C(=O)OCCOC(=O)OC(O)C(O)COC)C(O)COC</smiles>

(F)

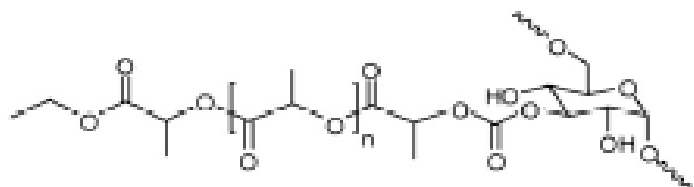

(H)

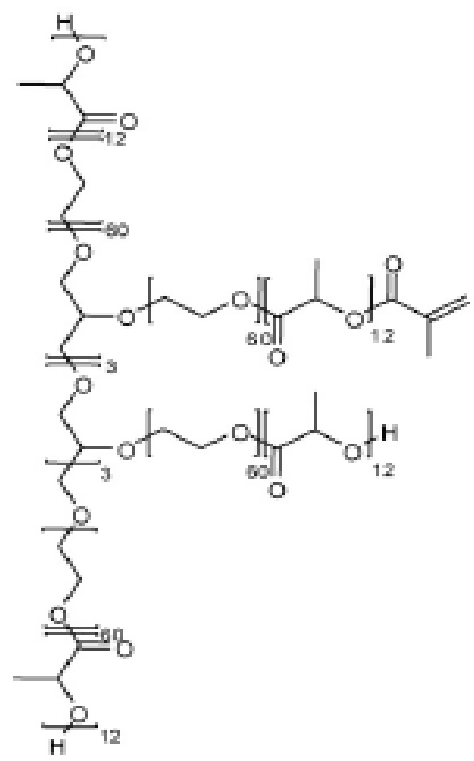

(B)

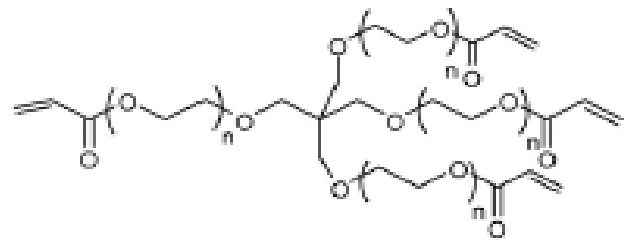

(E)

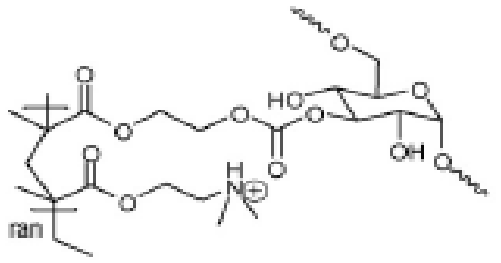

(G)

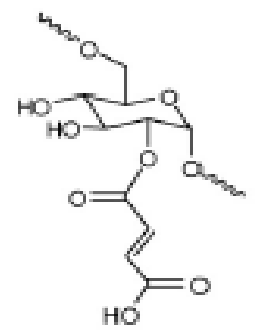

(I)

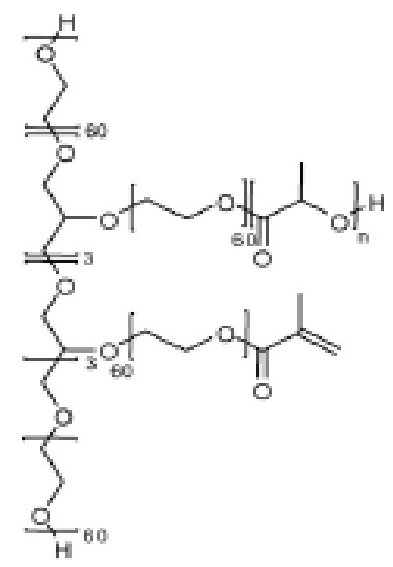


Figure 2.8.21 (A) PEG with oligo ( $\alpha$-hydroxy acids) and acrylate side groups, (B) acrylated 4-arm PEG, (C) star-shaped PEG-thioacetate, (D) dex-HEMA-MAA, (E) dex-HEMADMAEMA, (F) dex-lactate, $(\mathrm{G})$ dex-MA (dextran-maleic acid), (H) eight-arm PEG-PLA $2_{2-}$ MA start block copolymers, (I) PEG-MA/PLAn star block copolymers (From [205] ) polymerization rate and good cytocompatibility [213]. The mechanical properties could be

further increased by multimacromer polymerization, where interdigitating crosslinking networks could be created. This high crosslinking network will increase the mechanical properties of the gels, which will closely comparable to that of cartilage [213, 214].

\subsubsection{Non-viral vectors for gene delivery}

Conjugating a cell specific ligand to a drug loaded polyplex or micelle can enhance the targeted delivery of genes [29, 215]. The polymer-ligand complex has extraordinary potential as non-viral drug delivery vectors. Cationic polymers and polyelectrolytes have recently attracted as promising candidates for non-viral delivery vehicles due to their ability to self assemble and form complexes with plasmid DNA [216]. They are suitable for gene delivery systems when they are attached to ligands to mediate cell specific recognition and internalization and also due to their biocompatibility. Cancer treatment will be ideal with gene therapy once a safe, efficient synthetic gene delivery vector system is developed [216]. Suicide gene therapy for cancer employs a gene delivery vector to deliver DNA to cancer cells.

Successful gene therapy largely depends on the vector type that allows selective and efficient gene delivery to target cells with minimal toxicity. Nonviral vectors are much safer and cheaper, can be produced easily in large quantities, and have higher genetic material carrying capacity [216]. However, they are generally less efficient in delivering DNA and initiating gene expression as compared to viral vectors, particularly when used in vivo. As nonviral vectors, polycations may work well for efficient cell uptake and endosomal escape, 
because they do form compact and smaller complexes with plasmid DNA and carry amine groups, which give positive charge and buffering ability that allows safe escape from endosome/lysosome. Figure 2.22 [216] illustrates some of the potential candidates for polymer non-viral vectors.

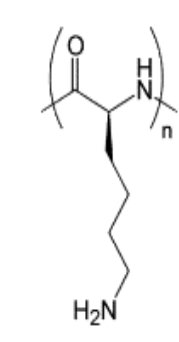

PLL

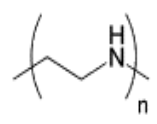

linear PEI

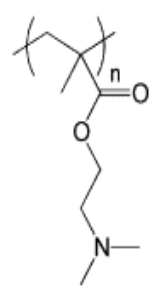

PDMAEMA

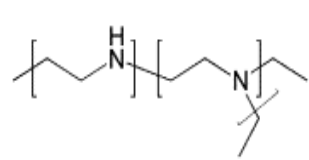

branched PEI

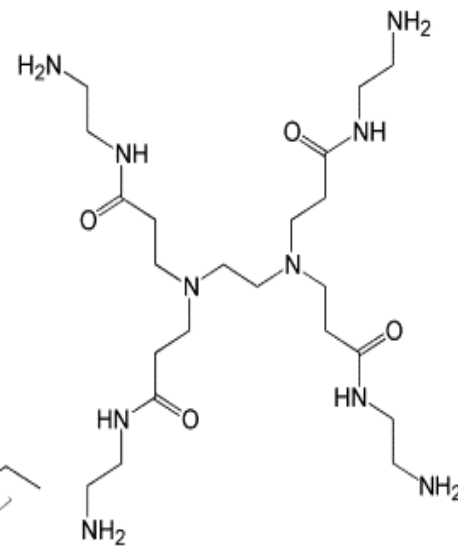

G1 PAMAM dendrimer

Figure 2.22. Polymer structures of some canditates for non-viral gene therapy. [216]

However, this is a disadvantage in the following step, which is releasing the plasmid DNA within the cytosol. In order to initiate transcription and enhance gene expression, the polymer/plasmid complex should dissociate after releasing from endosome safely and effectively. There are also other limitations with some of the polycationic carriers, for example, aggregation, toxicity, etc. Intelligent polymers, also called as 'stimuli responsive polymers', have a great potential as nonviral vectors to obtain site-, timing-, and duration period-specific gene expression, which is already exhibited in recent studies that are illustrated in Figure 22 [216]. In addition the ability of cationic peptide sequences to complex DNA and oligonucleotides offers prospects for the development of non-viral vectors for gene delivery, based on synthetic polymeric hybrid materials. Figure 2.23 is a simplified view of cellular uptake of polycation vectors complexed with DNA. 
The use of targeting ligands attached to the cationic polymers can allow for targeted uptake by selective cells through receptor-mediated endocytosis. These targeting ligands for tumors include epithelial growth factor (EGF), folate, transferrin etc. [216]. Since it has been found that epithelial growth factor (EGF) receptor is over expressed in many human tumors $[215,216]$, as an initial approach we can functionalize cationic stimuli responsive copolymers with natural EGF ligand for targeting tumor.

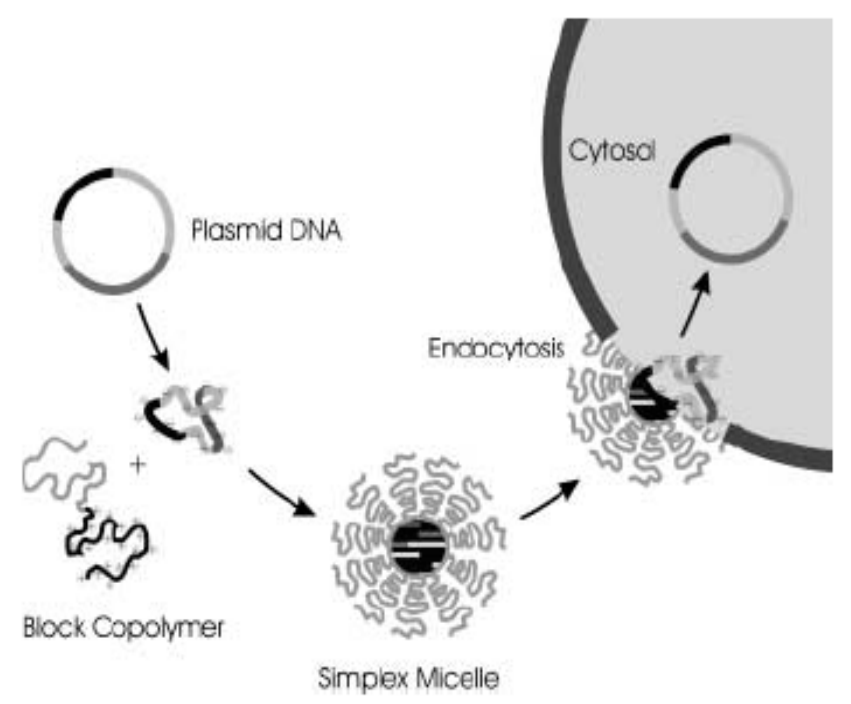

Figure 2.23. Cellular uptake path of polyplexes [1]

Several stimuli responsive smart copolymers and their potential applications in biomineralization and biomedical applications have been presented in this chapter. Several stimuli responsive block copolymers have been synthesized that have multi-responsive properties $[2,3]$. Most of the systems are limited to diblock and triblock copolymer systems due to the complex self-assembly and phase behavior of these materials $[3,4]$. There are few research studies on more complex systems such as pentablock copolymers with multi-stimuli responsive properties that can exhibit a rich phase behavior [133] and can serve as 
hierarchically assembling templates for biomineralization. In this work we are mainly focusing on Pluronic ${ }^{\circledR}$ based pentablock polymers with $\mathrm{pH}$, temperature and glucose responsive properties. The self-assembly and unique phase properties of these polymers make them potential candidates for several novel applications. Utilizing the self-assembling properties of these polymers for bottom-up approach for materials design similar to nature is a novel application in this respect. This approach of recreating nature materials from organic synthetic polymers also open up the future possibility of using synthetic biopolymers and their self-assembling properties for biomineralization as well. Furthermore, from previous studies it has been shown that a cationic Pluronic ${ }^{\circledR}$ based pentablock copolymer exhibit excellent biocompatibility [133], which provides the motivation to develop a family of selfregulating drug delivery formulations based on these systems. Finally, by tailoring these materials further, they can be used to develop insitu photocrosslinking hydrogel networks and to specifically target cancer cells in gene delivery which are also explored in this thesis work.

\subsection{References}

1. Forster, S. and T. Plantenberg, From self-organizing polymers to nanohybrid and biomaterials. Angewandte Chemie, International Edition, 2002. 41(5): p. 688-714.

2. $\quad$ Rodriguez-Hernandez, J., et al., Toward 'smart' nano-objects by self-assembly of block copolymers in solution. Progress in Polymer Science, 2005. 30(7): p. 691-724.

3. Ikkala, O. and G. ten Brinke, Hierarchical self-assembly in polymeric complexes: towards functional materials. Chemical Communications (Cambridge, United Kingdom), 2004(19): p. 2131-2137.

4. $\quad$ Foerster, S., Amphiphilic block copolymers for templating applications. Topics in Current Chemistry, 2003. 226(Colloid Chemistry I): p. 1-28.

5. Lum, K., D. Chandler, and J.D. Weeks, Hydrophobicity at Small and Large Length Scales. Journal of Physical Chemistry B, 1999. 103(22): p. 4570-4577.

6. Rosler, A., G.W. Vandermeulen, and H.A. Klok, Advanced drug delivery devices via self-assembly of amphiphilic block copolymers. Adv Drug Deliv Rev FIELD Full Journal Title:Advanced drug delivery reviews, 2001. 53(1): p. 95-108. 
7. Riess, G., Micellization of block copolymers. Progress in Polymer Science, 2003. 28(7): p. 1107-1170.

8. Desai, M., et al., Temperature and salt-induced micellization of some block copolymers in aqueous solution. Journal of Surfactants and Detergents, 2000. 3(2): p. 193-199.

9. Lee, A.S., et al., Structure of pH-Dependent Block Copolymer Micelles: Charge and Ionic Strength Dependence. Macromolecules, 2002. 35(22): p. 8540-8551.

10. Zheng, Y., et al., Directly Resolved Core-Corona Structure of Block Copolymer Micelles by Cryo-Transmission Electron Microscopy. Journal of Physical Chemistry B, 1999. 103(47): p. 10331-10334.

11. Wanka, G., H. Hoffmann, and W. Ulbricht, The aggregation behavior of poly(oxyethylene)-poly(oxypropylene)-poly(oxyethylene) block copolymers in aqueous solution. Colloid and Polymer Science, 1990. 268(2): p. 101-17.

12. Pedersen, J.S., et al., A Small-Angle Neutron and X-ray Contrast Variation Scattering Study of the Structure of Block Copolymer Micelles: Corona Shape and Excluded Volume Interactions. Macromolecules, 2003. 36(2): p. 416-433.

13. Pedersen, J.S., Analysis of small-angle scattering data from colloids and polymer solutions: modeling and least-squares fitting. Advances in Colloid and Interface Science, 1997. 70: p. 171-210.

14. Liu, J., T. Xu, and Y. Fu, Fundamental studies of novel inorganic-organic zwitterionic hybrids. 1. Preparation and characterizations of hybrid zwitterionic polymers. Journal of Non-Crystalline Solids, 2005. 351(37-39): p. 3050-3059.

15. Chu B, Z.Z., Physical chemistry of poly(oxyalkylene) block copolymer surfactants. . Non-ionic surfactants: polyoxyalkylene block copolymers, 1996. 60.

16. Yu, K. and A. Eisenberg, Bilayer Morphologies of Self-Assembled Crew-Cut Aggregates of Amphiphilic PS-b-PEO Diblock Copolymers in Solution.

Macromolecules, 1998. 31(11): p. 3509-3518.

17. Yu, G.-e. and A. Eisenberg, Multiple Morphologies Formed from an Amphiphilic ABC Triblock Copolymer in Solution. Macromolecules, 1998. 31(16): p. 5546-5549.

18. Liu, G., L. Qiao, and A. Guo, Diblock Copolymer Nanofibers. Macromolecules, 1996. 29(16): p. 5508-5510.

19. Stewart, S. and G. Liu, Block copolymer nanotubes. Angewandte Chemie, International Edition, 2000. 39(2): p. 340-344.

20. Cornelissen, J.J.L.M., et al., Helical superstructures from charged poly(styrene)poly(isocyanodipeptide) block copolymers. Science (Washington, D. C.), 1998. 280(5368): p. 1427-1430.

21. Yamamoto, H., et al., Polyion complex fiber and capsule formed by self-assembly of poly-L-lysine and gellan at solution interfaces. Journal of Applied Polymer Science, 2000. 79(3): p. 437-446.

22. Caruso, R.A., A. Susha, and F. Caruso, Multilayered Titania, Silica, and Laponite Nanoparticle Coatings on Polystyrene Colloidal Templates and Resulting Inorganic Hollow Spheres. Chemistry of Materials, 2001. 13(2): p. 400-409.

23. Bergbreiter, D.E., Self-assembled, sub-micrometer diameter semipermeable capsules. Angewandte Chemie, International Edition, 1999. 38(19): p. 2870-2872. 
24. Donath, E., et al., Novel hollow polymer shells by colloid-templated assembly of polyelectrolytes. Angewandte Chemie, International Edition, 1998. 37(16): p. 22022205.

25. Shchukin, D.G., G.B. Sukhorukov, and H. Moehwald, Smart inorganic/organic nanocomposite hollow microcapsules. Angewandte Chemie, International Edition, 2003. 42(37): p. 4472-4475.

26. Zhang, Y., et al., Fabrication of hollow capsules based on hydrogen bonding. Advanced Materials (Weinheim, Germany), 2003. 15(10): p. 832-835.

27. Alexandridis, P.a.B.L., Amphiphilic Block Copolymers: Self-Assembly and Applications. 2000.

28. Jeong, B. and A. Gutowska, Lessons from nature: stimuli-responsive polymers and their biomedical applications. Trends in Biotechnology, 2002. 20(7): p. 305-311.

29. Hoffmann, A.S., Really smart bioconjugates of smart polymers and receptor proteins. Sixth World Biomaterials Congress, 2000.

30. Gil, E.S. and S.M. Hudson, Stimuli-responsive polymers and their bioconjugates. Progress in Polymer Science, 2004. 29(12): p. 1173-1222.

31. Liu, S., N.C. Billingham, and S.P. Armes, A schizophrenic water-soluble diblock copolymer. Angewandte Chemie, International Edition, 2001. 40(12): p. 2328-2331.

32. Liu, S., et al., Synthesis of Shell Cross-Linked Micelles with $\mathrm{pH}$-Responsive Cores Using ABC Triblock Copolymers. Macromolecules, 2002. 35(16): p. 6121-6131.

33. Bories-Azeau, X., S.P. Armes, and H.J.W. Van den Haak, Facile Synthesis of Zwitterionic Diblock Copolymers without Protecting Group Chemistry. Macromolecules, 2004. 37(7): p. 2348-2352.

34. Weaver, J.V.M., S.P. Armes, and S. Liu, $A$ |"Holy Trinity|" of Micellar Aggregates in Aqueous Solution at Ambient Temperature: Unprecedented Self-Assembly Behavior from a Binary Mixture of a Neutral-Cationic Diblock Copolymer and an Anionic Polyelectrolyte. Macromolecules, 2003. 36(26): p. 9994-9998.

35. Shiomori, K., et al., Thermoresponsive properties of sugar sensitive copolymer of $N$ isopropylacrylamide and 3-(acrylamido)phenylboronic acid. Macromolecular Chemistry and Physics, 2004. 205(1): p. 27-34.

36. Zhou, Z.a.B.C., Phase Behavior and Association Properties of Poly(oxypropylene)Poly(oxyethylene)-Poly(oxypropylene) Triblock Copolymer in Aqueous Solution. Macromolecules, 1994. 27(8): p. 2025-33.

37. Lee, A.S., et al., Characterizing the Structure of pH Dependent Polyelectrolyte Block Copolymer Micelles. Macromolecules, 1999. 32(13): p. 4302-4310.

38. De Geest, B.G., et al., Glucose-Responsive Polyelectrolyte Capsules. Langmuir, 2006. 22(11): p. 5070-5074.

39. Lapeyre, V., et al., Monodispersed Glucose-Responsive Microgels Operating at Physiological Salinity. Biomacromolecules, 2006. 7(12): p. 3356-3363.

40. Liu, S. and S.P. Armes, Synthesis and Aqueous Solution Behavior of a pH-Responsive Schizophrenic Diblock Copolymer. Langmuir, 2003. 19(10): p. 4432-4438.

41. Rodriguez-Hernandez, J. and S. Lecommandoux, Reversible Inside-Out Micellization of pH-responsive and Water-Soluble Vesicles Based on Polypeptide Diblock Copolymers. Journal of the American Chemical Society, 2005. 127(7): p. 2026-2027. 
42. Szczubialka, K. and M. Nowakowska, Response of micelles formed by smart terpolymers to stimuli studied by dynamic light scattering. Polymer, 2003. 44(18): p. 5269-5274.

43. Peppas, N.A., et al., Hydrogels in biology and medicine: from molecular principles to bionanotechnology. Advanced Materials (Weinheim, Germany), 2006. 18(11): p. 1345-1360.

44. Deming, T.J., Polypeptide hydrogels via a unique assembly mechanism. Soft Matter, 2005. 1(1): p. 28-35.

45. Bures, P. and N.A. Peppas, Molecular dynamics of $\mathrm{pH}$-sensitive hydrogels based on poly(acrylic acid). Polymeric Materials Science and Engineering, 2000. 83: p. 506507.

46. Jeong, B., Y.H. Bae, and S.W. Kim, In situ gelation of PEG-PLGA-PEG triblock copolymer aqueous solutions and degradation thereof. J Biomed Mater Res FIELD Full Journal Title:Journal of biomedical materials research, 2000. 50(2): p. 171-7.

47. Peppas, N., Hydrogels in Medicine and Pharmacy. CRC, 1987.

48. Vakkalanka, S.K., C.S. Brazel, and N.A. Peppas, Temperature- and pH-sensitive terpolymers for modulated delivery of streptokinase. Journal of Biomaterials Science, Polymer Edition, 1996. 8(2): p. 119-129.

49. Zange, R., Y. Li, and T. Kissel, Biocompatibility testing of ABA triblock copolymers consisting of poly(L-lactic-co-glycolic acid) A blocks attached to a central poly(ethylene oxide) B block under in vitro conditions using different L929 mouse fibroblasts cell culture models. Journal of Controlled Release, 1998. 56(1-3): p. 249258.

50. Sawhney, S.A., Pathak, C. P., Hubbell, J. A., Biodegradable hydrogels based on photopolymerized poly(ethylene-glycol)-co-poly(alpha-hydroxy acid) diacrylate macromers. Macromolecules, 1993. 26: p. 581-587.

51. Kretlow, J.D., L. Klouda, and A.G. Mikos, Injectable matrices and scaffolds for drug delivery in tissue engineering. Advanced Drug Delivery Reviews, 2007. 59(4-5): p. 263-273.

52. Yoo, H.S., Photo-cross-linkable and thermo-responsive hydrogels containing chitosan and Pluronic for sustained release of human growth hormone (hGH). Journal of Biomaterials Science, Polymer Edition, 2007. 18(11): p. 1429-1441.

53. Martens, P. and K.S. Anseth, Characterization of hydrogels formed from acrylate modified poly(vinyl alcohol) macromers. Polymer, 2000. 41(21): p. 7715-7722.

54. Bryant, S.J., C.R. Nuttelman, and K.S. Anseth, The effects of crosslinking density on cartilage formation in photocrosslinkable hydrogels. Biomedical Sciences Instrumentation, 1999. 35: p. 309-314.

55. Nguyen, K.T. and J.L. West, Photopolymerizable hydrogels for tissue engineering applications. Biomaterials, 2002. 23(22): p. 4307-4314.

56. Zhang, K., M.R. Diehl, and D.A. Tirrell, Artificial Polypeptide Scaffold for Protein Immobilization. Journal of the American Chemical Society, 2005. 127(29): p. 1013610137.

57. Deming, T.J., Synthetic polypeptides for biomedical applications. Progress in Polymer Science, 2007. 32(8-9): p. 858-875. 
58. Nowatzki, P.J. and D.A. Tirrell, Physical properties of artificial extracellular matrix protein films prepared by isocyanate crosslinking. Biomaterials, 2003. 25(7-8): p. 1261-1267.

59. Link, A.J. and D.A. Tirrell, Reassignment of sense codons in vivo. Methods (San Diego, CA, United States), 2005. 36(3): p. 291-298.

60. Wang, L., et al., Expanding the genetic code of Escherichia coli. Science (Washington, DC, United States), 2001. 292(5516): p. 498-500.

61. Son, S., C. Tanrikulu, and D.A. Tirrell, Stabilization of bzip peptides through incorporation of fluorinated aliphatic residues. ChemBioChem, 2006. 7(8): p. 12511257.

62. Holmgren, S.K., et al., Code for collagen's stability deciphered. Nature (London), 1998. 392(6677): p. 666-667.

63. Kirshenbaum, K., S. Carrico Isaac, and A. Tirrell David, Biosynthesis of proteins incorporating a versatile set of phenylalanine analogues. Chembiochem FIELD Full Journal Title:Chembiochem : a European journal of chemical biology, 2002. 3(2-3): p. 235-7.

64. Nowak Andrew, P., et al., Rapidly recovering hydrogel scaffolds from selfassembling diblock copolypeptide amphiphiles. Nature FIELD Full Journal Title:Nature, 2002. 417(6887): p. 424-8.

65. Wang, X.S., R.A. Jackson, and S.P. Armes, Facile Synthesis of Acidic Copolymers via Atom Transfer Radical Polymerization in Aqueous Media at Ambient Temperature. Macromolecules, 2000. 33(2): p. 255-257.

66. Pyun, J., T. Kowalewski, and K. Matyjaszewski, Synthesis of polymer brushes using atom transfer radical polymerization. Macromolecular Rapid Communications, 2003. 24(18): p. 1043-1059.

67. Wang, X.S., et al., Facile synthesis of well-defined water-soluble polymers via atom transfer radical polymerization (ATRP) in aqueous media at ambient temperature. Chemical Communications (Cambridge), 1999(18): p. 1817-1818.

68. Save, M., et al., Atom Transfer Radical Polymerization of Hydroxy-Functional Methacrylates at Ambient Temperature: Comparison of Glycerol Monomethacrylate with 2-Hydroxypropyl Methacrylate. Macromolecules, 2002. 35(4): p. 1152-1159.

69. Matyjaszewski, K. and J. Xia, Atom Transfer Radical Polymerization. Chemical Reviews (Washington, D. C.), 2001. 101(9): p. 2921-2990.

70. McCormick, C.L., S.E. Kirkland, and A.W. York, Synthetic routes to stimuliresponsive micelles, vesicles, and surfaces via controlled/living radical polymerization. Polymer Reviews (Philadelphia, PA, United States), 2006. 46(4): p. 421-443.

71. Wager, C.M., D.M. Haddleton, and S.A.F. Bon, A simple method to convert atom transfer radical polymerization (ATRP) initiators into reversible addition fragmentation chain-transfer (RAFT) mediators. European Polymer Journal, 2004. 40(3): p. 641-645.

72. McCormick, C.L. and A.B. Lowe, Aqueous RAFT Polymerization: Recent Developments in Synthesis of Functional Water-Soluble (Co)polymers with Controlled Structures. Accounts of Chemical Research, 2004. 37(5): p. 312-325. 
73. Kricheldorf, H.R., $\alpha$-Aminoacid-N-Carboxyanhydrides and related materials Springe, Berlin Heidelberg New York, 1987.

74. Kricheldorf, H.R., Models of Biolpolymers by Ring-Opening Polymerization. Penczek S (ed), CRC Press, Boca Raton, FL, 1990.

75. Deming, T.J., Polypeptide and polypeptide hybrid copolymer synthesis via NCA polymerization. Advances in Polymer Science, 2006. 202(Peptide Hybrid Polymers): p. 1-18.

76. Stanely, S., Polymer synthesis and characterization methods. 1998.

77. Huglin, M.B., Light Scattering From Polymer Solutions. 1972.

78. Jhonson, C.S.a.D.A.G., Laser Light Scattering. 1981.

79. Berne, B. and R. Pecora, Dynamic light scattering: with applications to chemistry, biology and physics. 2000, New York: Wiley.

80. Feigin, L.A.a.D.I.S., Structure Ananlysis by Small-Angle X-Ray and Neutron Scattering. 1987.

81. Roe, R.-J., Methods of X-Ray and Neutron Scattering in Polymer Science. Topics in Polymer Science. 2000.

82. Guinier, A.a.G.F., Samm-Angle Scattering of X-Rays. 1985.

83. Castelletto, V.a.I.W.H., Small-Angle Scattering Functions of Micelles. Fibre Diffraction Review, 2003. 11: p. p. 36-43.

84. McConnell, G.A., et. al., , Disorder Order Transitions in Soft Sphere Polymer Micelles. Physical Review Letters, 1993. 71(13): p. p. 2102-2105.

85. Weyerich, B., J. Brunner-Popela, and O. Glarter, Small-angle scattering of interacting particles. II. Generalized indirect Fourier transformation under consideration of the effective structure factor for polydisperse systems. Journal of Applied Crystallography, 1999. 32(2): p. 197-209.

86. Gelhaaf, S.U., P. Schurtneberger, and M. Muller, New controlled environment vitrification system for cryo-trasmission electron microscopy: design and application to surfactant solution. Journal of Microscopy, 2000. 2: p. p.128.

87. Wang, H., et al., Syntheses of Amphiphilic Diblock Copolymers Containing a Conjugated Block and Their Self-Assembling Properties. Journal of the American Chemical Society, 2000. 122(29): p. 6855-6861.

88. Jain, S. and F.S. Bates, On the Origins of Morphological Complexity in Block Copolymer Surfactants. Science (Washington, DC, United States), 2003. 300(5618): p. 460-464.

89. Lam, Y.-M., N. Grigorieff, and G. Goldbeck-Wood, Direct visualization of micelles of Pluronic block copolymers in aqueous solution by cryo-TEM. Physical Chemistry Chemical Physics, 1999. 1(14): p. 3331-3334.

90. Won, Y.-Y., et al.,, Cryogenic transmission electron microscopy (Cryo-TEM) of micelles and vesicles formed in water by poly(ethylene oxide)-based block copolymers. American Chemical Society, 2001: p. p. A-K.

91. Wanka, G., H. Hoffmann, and W. Ulbricht, Phase Diagrams and Aggregation Behavior of Poly(oxyethylene)-Poly(oxypropylene)-Poly(oxyethylene) Triblock Copolymers in Aqueous Solutions. Macromolecules, 1994. 27(15): p. 4145-9.

92. Vadnere, M., et. al., , Thermodynamics studies on the gel-sol-transition of some Pluronic polyols.. Int.J. Pharm., 1984. 22(2-3): p. p.207-18. 
93. Petrov, A.I., A.A. Antipov, and G.B. Sukhorukov, Base-Acid Equilibria in Polyelectrolyte Systems: From Weak Polyelectrolytes to Interpolyelectrolyte Complexes and Multilayered Polyelectrolyte Shells. Macromolecules, 2003. 36(26): p. 10079-10086.

94. Pozzo, D.C., K.R. Hollabaugh, and L.M. Walker, Rheology and phase behavior of copolymer-templated nanocomposite materials. Journal of Rheology (New York, NY, United States), 2005. 49(3): p. 759-782.

95. Wang, P.a.T.P.J., Kinetics of sol-to gel transition for polosamer polyols. J. Appl. Polym. Sci, 1991. 43(2): p. p. 283-92.

96. Nystroem, B. and H. Walderhaug, Dynamic Viscoelasticity of an Aqueous System of a Poly(ethylene oxide)-Poly(propylene oxide)-Poly(ethylene oxide) Triblock Copolymer during Gelation. Journal of Physical Chemistry, 1996. 100(13): p. 5433-9.

97. Fujiwara, T., et al., Novel thermo-responsive formation of a hydrogel by stereocomplexation between PLLA-PEG-PLLA and PDLA-PEG-PDLA block copolymers. Macromolecular Bioscience, 2001. 1(5): p. 204-208.

98. Orkisz, M.J., et. al., Rheological properties of reverse thermogelling poly(acrylic acid)-g-(oxyethylene-b-oxypropylene-b-oxyethylene) polymers (SMART HYDROGEL). Book of Abstracts, 213th ACS National Meeting, San Francisco, 1997.

99. Wang, P.L.a.T.P.J., Sustained-release Interleukin-2 following intramuscular injection in rats. International Journal of Pharmaceutics, 1995. 113(1): p. 73-81.

100. Jeong, B., L.-Q. Wang, and A. Gutowska, Biodegradable thermoreversible gelling PLGA-g-PEG copolymers. Chemical Communications (Cambridge, United Kingdom), 2001(16): p. 1516-1517.

101. Sarikaya, M., et al., Materials assembly and formation using engineered polypeptides. Annual Review of Materials Research, 2004. 34: p. 373-408.

102. Coelfen, H. and S.-H. Yu, Biomimetic mineralization/synthesis of mesoscale order in hybrid inorganic-organic materials via nanoparticle self-assembly. MRS Bulletin, 2005. 30(10): p. 727-735.

103. Barthelat, F., Biomimetics for next generation materials. Philosophical Transactions of the Royal Society, A: Mathematical, Physical \& Engineering Sciences, 2007. 365(1861): p. 2907-2919.

104. Rao, P.R., Biomimetics. Sadhana, 2003. 28((3\&4)): p. p. 657-676.

105. Fratzl, P. and R. Weinkamer, Nature's hierarchical materials. Progress in Materials Science, 2007. 52(8): p. 1263-1334.

106. Coelfen, H. and S. Mann, Higher-order organization by mesoscale self-assembly and transformation of hybrid nanostructures. Angewandte Chemie, International Edition, 2003. 42(21): p. 2350-2365.

107. Belcher, A.M., et al., Control of crystal phase switching and orientation by soluble mollusk-shell proteins. Nature (London), 1996. 381(6577): p. 56-58.

108. Weiner, S. and L. Addadi, Design strategies in mineralized biological materials. Journal of Materials Chemistry, 1997. 7(5): p. 689-702.

109. Dabbs, D.M. and I.A. Aksay, Self-assembled ceramics produced by complex-fluid templation. Annu Rev Phys Chem FIELD Full Journal Title:Annual review of physical chemistry, 2000. 51: p. 601-22. 
110. Mann, S., The biomimetics of enamel: a paradigm for organized biomaterials synthesis. . Ciba Foundation Symposium, 1997. 205: p. 261-9.

111. van Bommel, K.J.C., A. Friggeri, and S. Shinkai, Organic templates for the generation of inorganic materials. Angewandte Chemie, International Edition, 2003. 42(9): p. 980-999.

112. Mann, S., Biomineralization, principles and concepts in bioinorganic materials chemistry. 2001.

113. www.brightminds.uq.edu.asu/teachers/resources/downloads/Grondahl.

114. Bauerlein, Biomineralization. Wiley-VCH, Weinheim, 2000.

115. Valery, C., et al., Biomimetic organization: Octapeptide self-assembly into nanotubes of viral capsid-like dimension. Proceedings of the National Academy of Sciences of the United States of America, 2003. 100(18): p. 10258-10262.

116. Klug, A., From macromolecules to biological assemblies. Angew.Chem.Int.Ed.Engl., 1983. 22: p. 565-636.

117. Nogales, E., et al., High-resolution model of the microtubule. Cell (Cambridge, Massachusetts), 1999. 96(1): p. 79-88.

118. Kabsch, W., Mannherz, H. G., Suck, D., Pai, E. F. \& Holmes, K. C., Atomic structure of the actin: DNase I complex Nature (London), 1990. 347: p. 37-44.

119. Holmes, K.C., Popp, D., Gebhard, W. \& Kabsch, W., Atomic model of the actin filament Nature (London), 1990. 347: p. 44-49.

120. Lehn, J.M., Angew.Chem.Int.Ed.Engl., 1990. 29: p. 1304-1319.

121. krejchi, M.T., Atkins, E.d., Waddon, A.J., Fournier, M.J., Mason, T.L. \& Tirrel, D. A., Chemical sequence control of beta sheet assembly in macromolecular crystals of periodic polypeptides. Science (Washington, D. C.), 1994. 265: p. 1427-1432.

122. Gulik-Kryzwicki, T., Fouquey, C. \& Lehn, J. M., Proc. Natl. Acad. Scie, 1993. 90: p. 163-167.

123. Bong, D.T., et al., Self-assembling organic nanotubes. Angewandte Chemie, International Edition, 2001. 40(6): p. 988-1011.

124. Aggell, A., et al., Responsive gels formed by the spontaneous self-assembly of peptides into polymeric b-sheets tapes. Nature (London), 1997. 386(6622): p. 259262.

125. Inouye, H., Fraser, P. E. \& Kirschner, D. A., Tetrameric assembly of full-sequence protein zero myelin glycoprotein by synchrotron x-ray scattering. Biophys. J., 1993. 64: p. 502-519.

126. Yagci, Y., Mishra, M. K., The polymeric materials encyclopedia. 1996.

127. Foerster, S., et al., Micellization of strongly segregated block copolymers. Journal of Chemical Physics, 1996. 104(24): p. 9956-9970.

128. Qin, A., et al., Polystyrene-poly(methacrylic acid) block copolymer micelles. Macromolecules, 1994. 27(1): p. 120-6.

129. Elias, H.G., Principles: Structure, synthesis and properties. Macromolecules, 1990. 1(5th edition).

130. Foerster, S., et al., Lyotropic Phase Morphologies of Amphiphilic Block Copolymers. Macromolecules, 2001. 34(13): p. 4610-4623.

131. Foerster, S., Borchert, U. and Lipprandt, U., Unpublished results. 
132. Mortensen, K., Phase behavior of poly(ethylene oxide)-poly(propylene oxide)poly(ethylene oxide) triblock copolymer dissolved in water. Europhys.Lett., 1992. 19(7): p. 599-604.

133. Determan, M.D., et al., Supramolecular Self-Assembly of Multiblock Copolymers in Aqueous Solution. Langmuir, 2006. 22(4): p. 1469-1473.

134. Chushak, Y. and A. Travesset, Coarse-grained molecular-dynamics simulations of the self-assembly of pentablock copolymers into micelles. Journal of Chemical Physics, 2005. 123(23): p. 234905/1-234905/7.

135. Khandpur, A.K., et al., Polyisoprene-Polystyrene Diblock Copolymer Phase Diagram near the Order-Disorder Transition. Macromolecules, 1995. 28(26): p. 8796-806.

136. Sanchez, C., et al., Biomimetism and bioinspiration as tools for the design of innovative materials and systems. Nature Materials, 2005. 4(4): p. 277-288.

137. Meyers, M.A., et al., Biological materials: Structure and mechanical properties. Progress in Materials Science, 2007. 53(1): p. 1-206.

138. Matyjaszewski, K., Controlled radical polymerization. Current Opinion in Solid State \& Materials Science, 1996. 1(6): p. 769-776.

139. Rizzardo, E., et al., Tailored polymers by free radical processes. Macromolecular Symposia, 1999. 143(World Polymer Congress, 37th International Symposium on Macromolecules, 1998): p. 291-307.

140. Chiefari, J., et al., Living Free-Radical Polymerization by Reversible AdditionFragmentation Chain Transfer: The RAFT Process. Macromolecules, 1998. 31(16): p. 5559-5562.

141. Zhang, S., Fabrication of novel biomaterials through molecular self-assembly. Nature Biotechnology, 2003. 21(10): p. 1171-1178.

142. Yu, S.-H., Bio-inspired crystal growth by synthetic templates. Topics in Current Chemistry, 2007. 271(Biomineralization II): p. 79-118.

143. Song, J., V. Malathong, and C.R. Bertozzi, Mineralization of Synthetic Polymer Scaffolds: A Bottom-Up Approach for the Development of Artificial Bone. Journal of the American Chemical Society, 2005. 127(10): p. 3366-3372.

144. Colfen, H. and L. Qi, A systematic examination of the morphogenesis of calcium carbonate in the presence of a double-hydrophilic block copolymer. Chemistry FIELD Full Journal Title:Chemistry (Weinheim an der Bergstrasse, Germany), 2001. 7(1): p. 106-16.

145. Failini, G., et al., Control of aragonite or calcite polymorphism by mollusk shell macromolecules. Science (Washington, D. C.), 1996. 271(5245): p. 67-9.

146. Antonietti, M., Surfactants for novel templating applications. Current Opinion in Colloid \& Interface Science, 2001. 6(3): p. 244-248.

147. Bronstein, L.M., et al., Interaction of metal compounds with 'double-hydrophilic' block copolymers in aqueous medium and metal colloid formation. Inorganica Chimica Acta, 1998. 280(1-2): p. 348-354.

148. Sidorov, S.N., et al., Stabilization of Metal Nanoparticles in Aqueous Medium by Poly(ethylene oxide)-Poly(ethylene imine) Block Copolymers. Journal of Colloid and Interface Science, 1999. 212(2): p. 197-211. 
149. Bronstein, L.M., et al., Induced Micellization by Interaction of Poly(2-vinylpyridine)block-poly(ethylene oxide) with Metal Compounds. Micelle Characteristics and Metal Nanoparticle Formation. Langmuir, 1999. 15(19): p. 6256-6262.

150. Yu, S.-H., H. Coelfen, and Y. Mastai, Formation and optical properties of gold nanoparticles synthesized in the presence of double-hydrophilic block copolymers. Journal of Nanoscience and Nanotechnology, 2004. 4(3): p. 291-298.

151. Zhang, D., et al., Formation of Silver Nanowires in Aqueous Solutions of a DoubleHydrophilic Block Copolymer. Chemistry of Materials, 2001. 13(9): p. 2753-2755.

152. Bouyer, F., et al., Role of double-hydrophilic block copolymers in the synthesis of lanthanum-based nanoparticles. Colloids and Surfaces, A: Physicochemical and Engineering Aspects, 2003. 217(1-3): p. 179-184.

153. Coelfen, H. and M. Antonietti, Crystal Design of Calcium Carbonate Microparticles Using Double-Hydrophilic Block Copolymers. Langmuir, 1998. 14(3): p. 582-589.

154. Marentette, J.M., et al., Crystallization of CaCO3 in the presence of PEO-blockPMAA copolymers. Advanced Materials (Weinheim, Germany), 1997. 9(8): p. 647651.

155. Yu, S.-H., et al., Biomimetic crystallization of calcium carbonate spherules with controlled surface structures and sizes by double-hydrophilic block copolymers. Advanced Functional Materials, 2002. 12(8): p. 541-545.

156. Rudloff, J., et al., Double-hydrophilic block copolymers with monophosphate ester moieties as crystal growth modifiers of $\mathrm{CaCO}$. Macromolecular Chemistry and Physics, 2002. 203(4): p. 627-635.

157. Kaluzynski, K., et al., Dihydrophilic block copolymers with ionic and nonionic blocks. I. Poly(ethylene oxide)-b-polyglycidol with $\mathrm{OP}(\mathrm{O})(\mathrm{OH}) 2, \mathrm{COOH}$, or $\mathrm{SO} 3 \mathrm{H}$ functions: synthesis and influence for $\mathrm{CaCO} 3$ crystallization. Journal of Polymer Science, Part A: Polymer Chemistry, 2001. 39(7): p. 955-963.

158. Yu, S.-H., H. Coelfen, and M. Antonietti, Polymer-Controlled Morphosynthesis and Mineralization of Metal Carbonate Superstructures. Journal of Physical Chemistry B, 2003. 107(30): p. 7396-7405.

159. Yu, S.-H., et al., Growth and Self-Assembly of BaCrO4 and BaSO4 Nanofibers toward Hierarchical and Repetitive Superstructures by Polymer-Controlled Mineralization Reactions. Nano Letters, 2003. 3(3): p. 379-382.

160. Enlow, D., et al., Synthesis and characterization of self-assembled block copolymer templated calcium phosphate nanocomposite gels. Journal of Materials Chemistry, 2007. 17(16): p. 1570-1578.

161. Busch, S., et al., Biomimetic morphogenesis of fluorapatite-gelatin composites. Fractal growth, the question of intrinsic electric fields, fore/shell assemblies, hollow spheres, and reorganization of denatured collagen. European Journal of Inorganic Chemistry, 1999(10): p. 1644-1653.

162. Kniep, R. and S. Busch, Biomimetic growth and self-assembly of fluorapatite aggregates by diffusion into denatured collagen matrixes. Angewandte Chemie, International Edition in English, 1996. 35(22): p. 2624-2626.

163. Imai, H., Y. Oaki, and A. Kotachi, A biomimetic approach for hierarchically structured inorganic crystals through self-organization. Bulletin of the Chemical Society of Japan, 2006. 79(12): p. 1834-1851. 
164. Oaki, Y. and H. Imai, Experimental Demonstration for the Morphological Evolution of Crystals Grown in Gel Media. Crystal Growth \& Design, 2003. 3(5): p. 711-716.

165. Sugawara, A., T. Ishii, and T. Kato, Self-organized calcium carbonate with regular surface-relief structures. Angewandte Chemie, International Edition, 2003. 42(43): p. 5299-5303.

166. Jongen, N., et al., Calcination and morphological evolution of cubic copper oxalate particles. Journal of Materials Science Letters, 2000. 19(12): p. 1073-1075.

167. Li, M., H. Schnablegger, and S. Mann, Coupled synthesis and self-assembly of nanoparticles to give structures with controlled organization. Nature (London), 1999. 402(6760): p. 393-395.

168. Gower, L.B. and D.J. Odom, Deposition of calcium carbonate films by a polymerinduced liquid-precursor (PILP) process. Journal of Crystal Growth, 2000. 210(4): p. 719-734.

169. Yu, S.-H., et al., Complex Spherical BaCO3 Superstructures Self-Assembled by a Facile Mineralization Process under Control of Simple Polyelectrolytes. Crystal Growth \& Design, 2004. 4(1): p. 33-37.

170. Peng, Y., et al., Polymer-Controlled Crystallization of Zinc Oxide Hexagonal Nanorings and Disks. Journal of Physical Chemistry B, 2006. 110(7): p. 2988-2993.

171. Shchukin, D.G., G.B. Sukhorukov, and H. Moehwald, Biomimetic Fabrication of Nanoengineered Hydroxyapatite/Polyelectrolyte Composite Shell. Chemistry of Materials, 2003. 15(20): p. 3947-3950.

172. Naka, K., et al., The effect of an anionic starburst dendrimer on the crystallization of CaCO3 in aqueous solution. Chemical Communications (Cambridge), 1999(19): p. 1931-1932.

173. Naka, K., Y. Tanaka, and Y. Chujo, Effect of Anionic Starburst Dendrimers on the Crystallization of CaCO3 in Aqueous Solution: Size Control of Spherical Vaterite Particles. Langmuir, 2002. 18(9): p. 3655-3658.

174. Estroff, L.A., C.D. Incarvito, and A.D. Hamilton, Design of a Synthetic Foldamer that Modifies the Growth of Calcite Crystals. Journal of the American Chemical Society, 2004. 126(1): p. 2-3.

175. Basko, M. and P. Kubisa, Properties of double-hydrophilic graft copolymers with a polyacetal backbone. Journal of Polymer Science, Part A: Polymer Chemistry, 2004. 42(5): p. 1189-1197.

176. Wegner, G., et al., Polymers designed to control nucleation and growth of inorganic crystals from aqueous media. Macromolecular Symposia, 2001. 175(Polymerization Processes and Polymer Materials II): p. 349-355.

177. Cha, J.N., et al., Biomimetic synthesis of ordered silica structures mediated by block copolypeptides. Nature FIELD Full Journal Title:Nature, 2000. 403(6767): p. 289-92.

178. Shenton, W., S.A. Davis, and S. Mann, Directed self-assembly of nanoparticles into macroscopic materials using antibody-antigen recognition. Advanced Materials (Weinheim, Germany), 1999. 11(6): p. 449-452.

179. Mao, C., et al., Viral assembly of oriented quantum dot nanowires. Proceedings of the National Academy of Sciences of the United States of America, 2003. 100(12): p. 6946-6951. 
180. Mirkin, C.A., Programming the Assembly of Two- and Three-Dimensional Architectures with DNA and Nanoscale Inorganic Building Blocks. Inorganic Chemistry, 2000. 39(11): p. 2258-2272.

181. Mirkin, C.A., et al., A DNA-based method for rationally assembling nanoparticles into macroscopic materials. Nature (London), 1996. 382(6592): p. 607-609.

182. Alivisatos, A.P., et al., Organization of 'nanocrystal molecules' using DNA. Nature (London), 1996. 382(6592): p. 609-611.

183. Dujardin, E., et al., DNA-driven self-assembly of gold nanorods. Chemical Communications (Cambridge, United Kingdom), 2001(14): p. 1264-1265.

184. Lee, S.-W., S.K. Lee, and A.M. Belcher, Virus-based alignment of inorganic, organic, and biological nanosized materials. Advanced Materials (Weinheim, Germany), 2003. 15(9): p. 689-692.

185. Roy, M.D., et al., Identification of a highly specific hydroxyapatite-binding peptide using phage display. Advanced Materials (Weinheim, Germany), 2008. 20(10): p. 1830-1836.

186. Chang, S., et al., Synthesis of a Potentially Bioactive, Hydroxyapatite-Nucleating Molecule. Calcified Tissue International, 2006. 78(1): p. 55-61.

187. Lee, J.-Y., et al., Assembly of collagen-binding peptide with collagen as a bioactive scaffold for osteogenesis in vitro and in vivo. Biomaterials, 2007. 28(29): p. 42574267.

188. Breulmann, M., et al., Elastic magnets. Template-controlled mineralization of iron oxide colloids in a sponge-like gel matrix. Advanced Materials (Weinheim, Germany), 1998. 10(3): p. 237-241.

189. Caruso, R.A., et al., Polymer gel template for porous TiO2 production. Berichte der Bunsen-Gesellschaft, 1998. 102(11): p. 1540-1543.

190. Velev, O.D., et al., Nanostructured porous materials templated by colloidal crystals: From inorganic oxides to metals. Book of Abstracts, 219th ACS National Meeting, San Francisco, CA, March 26-30, 2000, 2000: p. PHYS-425.

191. Subramanian, G., et al., Ordered macroporous materials by colloidal assembly. A possible route to photonic bandgap materials. Advanced Materials (Weinheim, Germany), 1999. 11(15): p. 1261-1265.

192. Peppas, N.A., et al., Physicochemical foundations and structural design of hydrogels in medicine and biology. Annual Review of Biomedical Engineering, 2000. 2: p. 929.

193. Ishihara, K., et. al., Glucose-induced permeation control of insulin through a complex membrane consisting of immobilized glucose oxidase and a polyamine. Polym.J. (Tokyo), 1984. 16(1): p. 625-31.

194. Gilbert, J.C.e.a., Drug release from Pluoronic F-127 gels. Int.J. Pharm., 1986. 32(23): p. p. 223-8.

195. Qiu, Y. and K. Park, Environment-sensitive hydrogels for drug delivery. Advanced Drug Delivery Reviews, 2001. 53(3): p. 321-339.

196. Schmaljohann, D., Thermo- and $\mathrm{pH}$-responsive polymers in drug delivery. Advanced Drug Delivery Reviews, 2006. 58(15): p. 1655-1670.

197. Schwarte, L.M.P., Kairali, Peppas, N. A., Cationic hydrogels for controlled release of proteins and other macromolecules. ACS Symposium Series, 1998. 709: p. p. 56-66. 
198. Ghandehari, H., P. Kopeckova, and J. Kopecek, In vitro degradation of $p H$-sensitive hydrogels containing aromatic azo bonds. Biomaterials, 1997. 18(12): p. 861-872.

199. Matsumoto, A., et al., Glucose-responsive polymer bearing a novel phenylborate derivative as a glucose-sensing moiety operating at physiological pH conditions. Biomacromolecules, 2003. 4(5): p. 1410-1416.

200. Matsumoto, A., R. Yoshida, and K. Kataoka, Glucose-responsive polymer gel bearing phenylborate derivative as a glucose-sensing moiety operating at the physiological pH. Biomacromolecules, 2004. 5(3): p. 1038-1045.

201. Podual, K., F.J. Doyle, and N.A. Peppas, Glucose-sensitivity of glucose oxidasecontaining cationic copolymer hydrogels having poly(ethylene glycol) grafts. Journal of Controlled Release, 2000. 67(1): p. 9-17.

202. Ehrick, J.D., et al., Genetically engineered protein in hydrogels tailors stimuliresponsive characteristics. Nature Materials, 2005. 4(4): p. 298-302.

203. Repo, R.U. and J.B. Finlay, Survival of articular cartilage after controlled impact. J Bone Joint Surg Am FIELD Full Journal Title:The Journal of bone and joint surgery. American volume, 1977. 59(8): p. 1068-76.

204. Nesic, D., et al., Cartilage tissue engineering for degenerative joint disease. Advanced Drug Delivery Reviews, 2006. 58(2): p. 300-322.

205. Van Tomme, S.R., G. Storm, and W.E. Hennink, In situ gelling hydrogels for pharmaceutical and biomedical applications. International Journal of Pharmaceutics, 2008. 355(1-2): p. 1-18.

206. Scranton, A.B., Bowman, C. N.m, Peiffer, R.W., Photopolymerization: Fundamentals and Applications. American Chemical Society, 1996.

207. Oudshoorn, M.H.M., et al., Synthesis and characterization of hyperbranched polyglycerol hydrogels. Biomaterials, 2006. 27(32): p. 5471-5479.

208. Wang, D.-a., et al., Synthesis and characterization of a novel degradable phosphatecontaining hydrogel. Biomaterials, 2003. 24(22): p. 3969-3980.

209. Li, Q., et al., Biodegradable and photocrosslinkable polyphosphoester hydrogel. Biomaterials, 2006. 27(7): p. 1027-1034.

210. Davis, K.A., J.A. Burdick, and K.S. Anseth, Photoinitiated crosslinked degradable copolymer networks for tissue engineering applications. Biomaterials, 2003. 24(14): p. 2485-2495.

211. Fisher, J.P., et al., Photoinitiated cross-linking of the biodegradable polyester poly(propylene fumarate). Part I. Determination of network structure. Biomacromolecules, 2003. 4(5): p. 1327-1334.

212. Timmer, M.D., C.G. Ambrose, and A.G. Mikos, Evaluation of thermal- and photocrosslinked biodegradable poly(propylene fumarate)-based networks. Journal of Biomedical Materials Research, Part A, 2003. 66A(4): p. 811-818.

213. Martens, P.J., S.J. Bryant, and K.S. Anseth, Tailoring the Degradation of Hydrogels Formed from Multivinyl Poly(ethylene glycol) and Poly(vinyl alcohol) Macromers for Cartilage Tissue Engineering. Biomacromolecules, 2003. 4(2): p. 283-292.

214. Khan, Y., et al., Tissue engineering of bone: material and matrix considerations. J Bone Joint Surg Am FIELD Full Journal Title:The Journal of bone and joint surgery. American volume, 2008. 90 Suppl 1: p. 36-42. 
215. Ogris, M., et al., Tumor-targeted gene therapy: strategies for the preparation of ligand-polyethylene glycol-polyethylenimine/DNA complexes. Journal of Controlled Release, 2003. 91(1-2): p. 173-181.

216. Arap, W., R. Pasqualini, and E. Ruoslllahti, Cancer treatment by targeted drug delivery to tumor vasculature in a mouse model. Science (Washington, D. C.), 1998. 279(5349): p. 377-380. 


\section{CHAPTER 3. RESEARCH OBJECTIVES}

\subsection{Introduction}

The main goal of this thesis work is to develop novel smart block copolymers that are responsive to temperature as well as to $\mathrm{pH}$ or glucose, which can be used for biomineralization and biomedical applications. Self-assembling, thermo reversible ionic block copolymers have been developed for use as templates for bottom-up synthesis of materials mimicking natural processes. Bottom-up synthesis of macroscale ordered nanocomposites from self-assembling hierarchically ordered polymer templates is a novel contribution to materials design. Future directions of this work will involve the development of biopolymers and synthetic polypeptides as templates for this bottom-up approach to materials design. Temperature, $\mathrm{pH}$ and glucose responsive pentablock copolymers as well as crosslinkable hydrogel networks have also been developed with consideration towards injectable glucose-sensitive insulin delivery applications, polymer vectors for non-viral gene therapy and tissue engineering applications.

\subsection{Specific goals of the research}

\subsubsection{Develop block copolymer templates for biomineralization}

- Develop novel ionic pentablock copolymers and block copolymers with mineral binding peptide conjugates with $\mathrm{pH}$ and temperature sensitive properties. Atom transfer radical polymerization (ATRP) is used as the synthesis method, yielding low polydispersity and controlled copolymer architecture.

- Characterize the aqueous phase behavior of these polymers using NMR, DSC, SAXS, SANS and dynamic light scattering.

- Investigate the potential of this family of ionic copolymers as templates for 
biomineralization. A novel synthesis route for materials design by using the selfassembling and thermoreversible properties of these polymers for a bottom-up approach for nanocomposite synthesis is made possible.

- Characterize the self-assembled organic-inorganic nanocomposite superstructure by small angle scattering, XRD, NMR, TGA, rheology and electron microscopy methods.

This is a collaborative project and the work is presented in Chapters 4,5 and 6. This work will serve as a good platform for future directions of this project, where the focus will be on the development of artificial polypeptide scaffolds for templated mineralization

\subsubsection{Applications of these smart block copolymers in the biomedical field}

- Develop novel glucose sensitive pentablock copolymers. Investigate the potential of these pentablock copolymers for future in vitro dynamic insulin release studies from these copolymer depot formulations. This work is presented in Chapter 7.

- Develop thermoresponsive injectable photocrosslinking hydrogles for posttraumatic cartilage repair. This work is presented in Chapter 8 .

The intent of this work is to provide a basic platform for future design of novel nanocomposite materials, that will have excellent properties similar to that of nature, from a bottom-up approach using novel self-assembling synthetic polymer templates as well as further tailoring of these family of block copolymers to contribute to biomedical applications such as drug delivery, non-viral gene therapy and tissue engineering. 


\section{CHAPTER 4. SYNTHESIS AND CHARACTERIZATION OF SELF-}

\section{ASSEMBLED BLOCK COPOLYMER TEMPLATED CALCIUM PHOSPHATE NANOCOMPOSITE GELS}

(This chapter is based on collaborative work published in Journal of Materials Chemistry [19] and has been modified to focus mainly on my contributions to this work.)

D. Enlow ${ }^{\mathrm{a}}$, A. Rawal $^{\mathrm{b}}$, M. Kanapathipillai ${ }^{\mathrm{c}}$, M. Akinc ${ }^{\mathrm{a}}$, K. Schmidt-Rohr ${ }^{\mathrm{b}}$, C.T.Lo ${ }^{\mathrm{d}}$, P. Thiyagarajan $^{\mathrm{e}}$, Surya K. Mallapragada ${ }^{\mathrm{c}}$

\subsection{Abstract}

Bioinspired materials are of particular interest because of their possible implications in biomedical, and in the nano-manufacturing field. The study of the mechanisms involved in bonelike mineral growth on organic polymer templates is an emerging field in this respect. Amphiphilic block copolymers which self assemble to form macroscopic gels in response to $\mathrm{pH}$ and temperature are ideal candidates as hierarchically assembling templates for biomimetic materials research. In this study, amphiphilic block copolymer micelles in aqueous solutions were used as templates for the growth of calcium phosphate nanocrystals and subsequently allowed to self-assemble to form thermoreversible nanocomposite gels at low $\mathrm{pH}$ conditions. Pluronic ${ }^{\circledR}$ F127 copolymers and poly(N, N-(diethylamino ethyl methacrylate) modified Pluronic ${ }^{\circledR}$ F127 cationic pentablock copolymers with pka 7.4 were used as templates in this study. Small angle X-ray scattering (SAXS) measurements along with X-ray diffraction (XRD), Nuclear magnetic resonance (NMR), Transmission electron microscopy (TEM) experiments were performed to study the ordered polymer-based nanocomposite superstructure. SAXS measurements showed distinct peaks with $Q / Q^{*}\left(Q^{*}\right.$ is 
the first order diffraction peak position of $\sqrt{3}: \sqrt{4}: \sqrt{8}: \sqrt{11}: \sqrt{12}$ corresponding to a face centered cubic structure (FCC) of Pluronic ${ }^{\circledR}$ F127 polymer nanocomposite gel structures. The pentablock copolymer templates on the other hand, showed broader scattering peaks. But the trends resembled the Pluronic ${ }^{\circledR}$ SAXS patterns indicating a poorly ordered FCC structure. Further the scattering patterns and the length scales for both block copolymer nanocomposite structures indicated that the calcium phosphate precipitated on and interacted with the polymer micelles forming an organized network of $\sim 20 \mathrm{~nm}$ diameter nanospheres which is in agreement with solid state NMR findings. To conclude, successful mineralization of calcium phosphate was demonstrated by a novel polymer-inorganic self-assembly synthesis scheme similar to that seen in nature.

\footnotetext{
${ }^{a}$ Department of Materials Engineering, Iowa State University and Ames Laboratory ${ }^{b}$ Department of Chemistry, Iowa State University and Ames Laboratory ${ }^{c}$ Department of Chemical Engineering, Iowa State University and Ames Laboratory dAdvanced Photon Source, Argonne National Laboratory ${ }^{\mathrm{e}}$ Intense Pulsed Neutron Source, Argonne National Laboratory
}

\subsection{Introduction}

Design of new materials synthesis for various engineering applications has recently been based on mimicking natural structures and processes due to their intriguing structural and mechanical properties. Bones, the dentine, eggshells and the shells of marine mollusks are some examples of highly complex organic-inorganic nanocomposite materials produced in nature by self-assembly. These naturally occurring materials contain different components in defined hierarchical structures, and over several length scales [1], where both the composition and the structure are responsible for the micro and macroscopic properties. 
Novel biomimetic approaches offer the possibility not only to recreate these natural biomaterials, but also provide the flexibility to control the architecture (the structure) and the chemistry (the composition) of these materials design [2].

In recent years there has been considerable progress in the use of block copolymers as templates for the controlled synthesis of nano particles and mesoporous inorganic materials [3]. The template has the advantage that its size and shape can be reproducibly controlled, in contrast to the desired material, where such control is much more difficult. In designing new materials, Nature provides many exciting sources of ideas and inspiration. The study of the mechanisms and factors of bonelike mineral growth on organic polymer templates has great potential in the fields of biomimetics and biomineralization. In nature, bones form first as self assembling matrices of collagen fibrils. By forming bundles of triple helix fibers and larger assemblies of layers or lamellae, the collagen protein acts as the guide for hydroxyapatite precipitation[4]. Hydroxyapatite (stable form of calcium phosphate at physiological $\mathrm{pH}$ ) forms on the collagen surfaces and gives the bone its rigidity and shape.

Several templating methods have been developed to mimic the natural process of calcium phosphate nanocrystal formation. Muller et al investigated biomimetic apatite formation on chemically modified cellulose templates [5], where directed calcium phosphate precipitation on the surfaces of the cellulose fibers was observed with an initial diameter of about $20 \mu \mathrm{m}$. These coated fibers reached a final diameter of over $90 \mu \mathrm{m}$. Similarly, Song et al developed a procedure using poly(2-hydroxyethyl methacrylate) (pHEMA) hydrogel scaffolds $[6,7]$. In a similar study, Song et al were able to precipitate calcium phosphate onto the surface of hydrogel strips. In this case, urea was used to slowly raise the solution $\mathrm{pH}$ and induce precipitation of calcium phosphate on the carboxylate-rich pHEMA surfaces. 
The result was a poorly crystalline $\sim 5 \mu \mathrm{m}$ thick coating of hydroxyapatite over the surface of the hydrogel strip. A bimodal crystallite size distribution was observed with 15-20 nm primary crystallites and larger "cluster-like" domains of 200-400 nm. On a smaller scale, Rusu et al used chitosan as a natural biopolymer matrix for their hydroxyapatite nanoparticle composites [8]. By mixing calcium chloride and sodium dihydrogen phosphate with a solution of chitosan in water, they were able to show self-assembly and size control as the hydroxyapatite crystallites formed inside the chitosan matrix. Recently Tanashi et al. showed, based on studies on self-assembled monolayers, that ionic templates are better for calcium phosphate precipitation [9]. Further Zhao et al. recently reported using F127 polymer templates for hydroxyapatite crystal synthesis, where rod like particles of 100- $200 \mathrm{~nm}$ size were obtained by co-precipitation of different concentration of the polymer-inorganic mixtures at high temperatures[10].

While all of these studies produce biomimetic polymer-inorganic composites, the polymer-inorganic interaction is limited for the most part to the surface of the polymer. By using concentrated gel forming polymers with similar approach, it should be possible to precipitate calcium phosphate throughout a network of agglomerated polymer nanospheres. Self assembling smart copolymers could be used as nanostructure templates to understand how calcium phosphate precipitates onto self-assembling synthetic polymer micelles [11]. By understanding how calcium phosphate precipitates onto self-assembling synthetic polymer micelles, we can try to mimic the natural bone like materials in a bottom-up approach. In this study we have used Pluronic ${ }^{\circledR}$ F127 copolymers and Poly (N, N-(diethylamino) ethyl methacrylate) modified Pluronic ${ }^{\circledR}$ F127 cationic pentablock copolymers with pka $\sim 7.4$ as our polymer templates. 
The phase behavior of polyethylene oxide (PEO) and polypropylene oxide (PPO) block copolymers, commercially known as Pluronic ${ }^{\circledR}$ or Poloxamer ${ }^{\circledR}$, have been extensively studied over the past decade [12]. Both PEO and PPO blocks are water soluble at low temperatures $\left(<5^{\circ} \mathrm{C}\right)$ and concentrated fluid dispersions are easily created. The PPO block becomes increasingly dehydrated and the polymers self-associate into micellar aggregates above room temperature due to their lower critical solution temperature (LCST) characteristics. This transition is fully reversible on repeated heating and cooling. At large polymer concentrations, the spherical micelles pack into micelle cubic crystals, and the Pluronic ${ }^{\circledR}$ F127 is known to form cubic structures of spherical micelles with a solid PPO core and a hydrated PEO corona [13]. We use this model system in our work due to its thermoreversible transition and its extensively characterized solution behavior [12], [13], and [14].

Previously in our group, an amphiphilic polycationic pentablock copolymer, poly(2diethylaminoethyl-methylmethacrylate)-poly(ethyleneoxide)-poly(propyleneoxide)poly(ethyleneoxide)-poly(2-diethylaminoethyl-methylmethacrylate)PDEAEM $25-\mathrm{PEO}_{100^{-}}$ $\mathrm{PPO}_{65}-\mathrm{PEO}_{100}-\mathrm{PDEAEM}_{25}$ ) was synthesized by atom transfer radical polymerization [15]. This smart copolymer was mainly designed to exhibit thermoreversible gelation, slower dissolution compared to Pluronic ${ }^{\circledR}$ gels [23], as well as to exhibit pH dependent solubility around physiological $\mathrm{pH}$ due to its $\mathrm{pKa}$ value.

While previous studies were limited to calcium phosphate-polymer interactions on the surface of a bulk polymer, or fractions of a micron agglomerates of nanocomposites from solution, these polymers make it possible to precipitate calcium phosphate in the interstitial spaces between and on the surface of the concentrated spherical nanoscale micelles within a 
macro-sized polymer gel. The temperature dependent transition of these polymers makes it possible to combine the polymer and calcium phosphate components in solution, allowing for a homogeneous mixture of the micelle spheres and calcium phosphate. The $\mathrm{pH}$ sensitivity of the pentablock copolymer at low $\mathrm{pH}$ further enhances the organic-inorganic ionic-interaction induced precipitation, facilitating high percentage of inorganic phase formation on the organic polymer template.

\subsection{Materials and Methods}

\subsubsection{Polymer synthesis method and scheme}

Unless otherwise noted, all inorganic chemicals in this study were obtained from Fisher Scientific and all organic chemicals were obtained from Sigma Aldrich and are of laboratory grade and purity. The cationic pentablock copolymer was synthesized by ATRP as previously described [15]. Briefly, first the Pluronic ${ }^{\circledR}$ macroinitiator was prepared as reported previously [15]. Then the difunctional Pluronic ${ }^{\circledR}$ macroinitiator $(8 \mathrm{~g}, 0.06$ mmole $)$, copper bromide $(0.1885 \mathrm{~g}, 0.06 \mathrm{mmole})$, and toluene $(50 \mathrm{ml})$, were added to a flask, and the solution was stirred well. Then a small amount of copper was added to the reaction to enhance the stability of the $\mathrm{Cu}(\mathrm{I}) / \mathrm{Cu}(\mathrm{II})$ equilibrium. After stirring, the flask was sealed with a septum. The toluene suspension was purged with Argon for $5 \mathrm{~min}$. DEAEM (0.885 g, $2.1 \mathrm{mmole})$ was added through the septum with a syringe. After stirring the mixture, N-ppm ligand (0.37 g, 0.12 mmole) was then added via syringe. The mixture was freeze pumped thawed thrice and filled with Argon. The reaction was then carried out at $70^{\circ} \mathrm{C}$ in an oil bath overnight. The reaction mixture was then diluted in methylene chloride and passed through a short alumina column to remove copper catalyst and was rotary evaporated. The remaining mixture was 
precipitated in n-heptane and dried under vacuum. The synthesis scheme is shown in Figure

\section{1.}

\section{Synthesis scheme:}
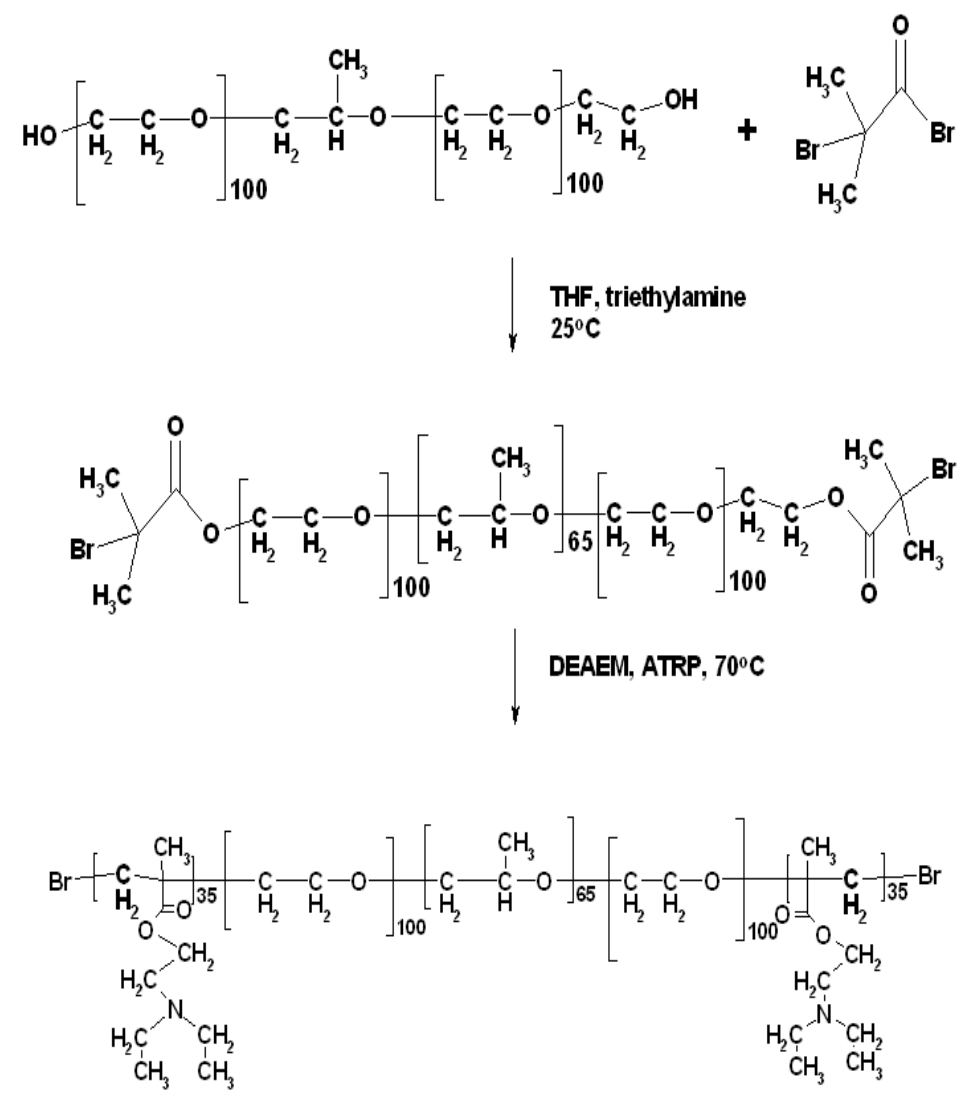

Figure 4.1. Synthesis scheme of DEAEM pentablock copolymer

The molecular weight and the composition of the polymer is determined by ${ }^{1} \mathrm{HNMR}$ and GPC. The thermo reversible gelation of the polymer is determined by simple tube inversion technique. After polymer characterization polymer-inorganic nanocomposite synthesis was carried out.

\subsubsection{Polymer-inorganic gel nanocomposite synthesis}

Polymer gel nanocomposites were prepared by utilizing the self-assembling properties of polymer due to $\mathrm{pH}$ and temperature responsiveness. The nanocomposite 
synthesis was carried out by collaborators in the Akinc laboratory. A brief description of the nanocomposite synthesis method developed by Enlow et al. is provided below.

Precipitation of calcium phosphate into the polymer gel matrix was achieved using aqueous solutions of ammonium dihydrogen phosphate $\left(\mathrm{NH}_{4} \mathrm{H}_{2} \mathrm{PO}_{4}\right)$, phosphoric acid $\left(\mathrm{H}_{3} \mathrm{PO}_{4}\right)$, calcium nitrate $\left(\mathrm{Ca}\left(\mathrm{NO}_{3}\right)_{2}\right)$, and the PDEAEM modified pentablock polymer. Saturated solution of calcium phosphate with a $\mathrm{Ca}: \mathrm{P}$ ratio of 1.67 as in stoichiometric hydroxyapatite was prepared by mixing $120 \mathrm{~mL}$ of a $0.5 \mathrm{M}$ solution of ammonium $\mathrm{NH}_{4} \mathrm{H}_{2} \mathrm{PO}_{4}$ with $200 \mathrm{~mL}$ of a $0.5 \mathrm{M} \mathrm{Ca}\left(\mathrm{NO}_{3}\right)_{2}$ solution. Later, it was stirred for 30 minutes until a white precipitate formed. The mixture was then centrifuged and the clear supernatant was drawn off as the saturated calcium phosphate solution. This supernatant solution had a $\mathrm{pH}$ of 2.95 . Similarly, a more concentrated solution was prepared by mixing $4.0 \mathrm{M}$ solutions of $\mathrm{H}_{3} \mathrm{PO}_{4}$ and $\mathrm{Ca}\left(\mathrm{NO}_{3}\right)_{2}$. Sodium hydroxide $(\mathrm{NaOH})$ was added until the solution reached a $\mathrm{pH}$ of 1.0 and a cloudy precipitate formed. Like the dilute solution described above, the supernatant was saved as the saturated calcium phosphate solution at $\mathrm{pH}=1.0$.

Gel samples were prepared by dissolving 3 grams of the pentablock copolymer directly into the saturated calcium phosphate solutions. Control samples of the polymer dissolved in deionized water were also prepared. These mixtures were placed in a refrigerator at $3^{\circ} \mathrm{C}$ and stirred daily until the polymer was completely dissolved. It took usually about 3 to 4 days to get a homogeneous solution. After dissolving, the samples were warmed to room temperature $\left(22^{\circ} \mathrm{C}\right)$ and aged for 24 hours to enable the polymer solution to form a gel. During this time, as predicted by the phase behavior of these polymers, the calcium phosphate-polymer solution thickened into a viscous nanocomposite gel.

\subsection{Characterization of polymer-gel nanocomposites:}


Polymer characterization, SAXS measurements along with XRD, TGA, NMR and TEM experiments performed by collaborators in the Akinc and Schmidt-Rohr groups were used to study the ordered polymer based nanocomposite superstructure. Briefly, NMR and GPC measurements were performed to determine the composition and molecular weights of the block copolymers. Cryo TEM measurements were used to visualize the nanostructure of the copolymer gels and micelles without disrupting the copolymer structure. TGA measurements were performed to obtain the percentage of inorganic in the nanocomposite. SAXS measurements were used to find the structural order and length scale of polymer gel structure. Solid state NMR is a powerful technique that can provide useful information on inorganic composition and the size of the nanostructures formed. Cryo TEM, SAXS and solid state NMR are complementary techniques to study the structure and size of the composites. XRD measurements were used to identify the crystalline phase of the inorganic and as well as the size. XRD and solid state NMR are complementary techniques to study the inorganic phase.

For polymer characterization all ${ }^{1} \mathrm{H}$ NMR measurements were performed using a Varian VXR400 (400 MHz) spectrometer and the chemical shifts are given in ppm. $\mathrm{D}_{2} \mathrm{O}$ and Chloroform-d were the solvents used for the measurements. Molecular weight and polydispersity index (PDI) of the copolymers were estimated using a PLgel 100, 500, 1x10 $1 \times 10^{5} \AA$ at $40^{\circ} \mathrm{C}$ equipped with a Waters 510 pump, Waters 717 autosampler, a Wyatt Optilab DSP refractometer, and a Wyatt Dawn EOS light scattering detector

Cryo-TEM measurements were performed to visualize the gel nanostructure, as it is necessary to retain the copolymer micelle/gel structures. Nanocomposite gel samples were 
prepared as described above, placed onto bulls-eye stub, and frozen at $-100^{\circ} \mathrm{C}$ in the chamber of a Reichert Ultracut S ultramicrotome with FCS cryo unit (Mager Scientific Inc., Dexter MI). Using a Diatome cryo-diamond knife ( $35^{\circ}$-dry; Electron Microscopy Sciences, Ft. Washington, PA), sections were made at $100 \mathrm{~nm}$ and collected onto 300 mesh copper grids and placed into a grid transfer unit that was stored in liquid nitrogen until transferred to the TEM chamber. Later, the TEM samples were loaded into a liquid nitrogen cooled Gatan cold stage (Model 626DH, Gatan Inc. Pleasanton, CA) and imaged at $100 \mathrm{kV}$ in a Phillips CM 30 TEM (Phillips Corporation, Schaumburg, IL).

Liquid copolymer solutions were prepared by placing $50 \mu \mathrm{L}$ of the aqueous sample onto formvar coated copper grids for 1 minute, allowing contents to settle. After getting rid of the supernatant, $1 \%$ phosphotungstic acid ( $\mathrm{pH}$ 6.2) was applied for 30 seconds as a negative contrast stain. Later, the grid was wicked and allowed to dry. Images were captured on a JEOL 1200EX II scanning transmission electron microscope (Japan Electron Optic Laboratories, Peabody, MA) using a Megaview III digital camera and SIS Pro. Software (Soft Imaging Systems Inc., LLC, Lakewood, CO).

Small angle X-ray scattering (SAXS) experiments were performed on the Pluronic ${ }^{\circledR}$ F127 and pentablock copolymer gels with and without the inorganic phase using the instrument at the 12-ID beam line at the Advanced Photon Source in Argonne National Laboratory to elucidate the gel-nanostructure. A $15 \times 15 \mathrm{~cm}$ detector was used to measure the scattered intensity and the transmitted intensity was measured using a photodiode. Samples were held in DSC pans for solids, $2 \mathrm{~m}$ from the detector. The beam energy used for the analysis is $12 \mathrm{keV}(\lambda=1.035 \AA)$ and the data were collected at $1 \mathrm{~s}$ exposure. The collected 2D data were corrected, azimuthally averaged, and placed on an absolute scale. 
X-ray diffraction experiments were used to characterize the structure of the inorganic using a theta-theta x-ray diffractometer (Scintag, XGEN-400, and Cupertino, CA). Wet gel XRD samples were prepared and analyzed immediately to minimize drying. The $\mathrm{Cu} \mathrm{K} \alpha \mathrm{X}-$ ray source was set to $45 \mathrm{kV}$ and $40 \mathrm{~mA}$, and the samples were scanned at a rate of $1 \%$ min over a $2 \theta$ range of $10^{\circ}-70^{\circ}$. Phase analysis was done using the ICDD database and the Scintag DMSNT search / match software.

Thermal gravimetric analysis (TGA) performed with a Perkin Elmer thermo gravimetric analyzer (Perkin Elmer, TGA 7, and Downers Grove IL). Approximately 40mg of the gel sample was placed in a platinum pan and the experiments were performed in a flowing air environment. The program was set to heat up to $100^{\circ} \mathrm{C}$ and hold at this temperature for $10 \mathrm{~min}$, then heat from $100^{\circ} \mathrm{C}$ to $150^{\circ} \mathrm{C}$ at a rate of $3.00^{\circ} \mathrm{C} / \mathrm{min}$, and finally, heat from $150^{\circ} \mathrm{C}$ to $800^{\circ} \mathrm{C}$ at rate of $10^{\circ} \mathrm{C} / \mathrm{min}$.

All NMR experiments were carried out on a Bruker spectrometer (Bruker-Biospin DSX400, Rheinstetten, Germany) at $400 \mathrm{MHz}$ for ${ }^{1} \mathrm{H}$ and $162 \mathrm{MHz}$ for ${ }^{31} \mathrm{P}$ nuclei. Direct polarization (DP) and cross polarization (CP) ${ }^{31} \mathrm{P}$ NMR spectra were acquired with ${ }^{1} \mathrm{H}$ decoupling at $6.5 \mathrm{kHz}$ MAS. All ${ }^{1} \mathrm{H}$ NMR spectra were recorded at $6.5 \mathrm{kHz}$ MAS using probe-head background suppression [16]. The line-width of the proton spectrum indicates the ${ }^{1} \mathrm{H}-{ }^{1} \mathrm{H}$ dipolar coupling, which increases with the proton density and decreases with the mobility of the segment.

Supramolecular proximities and domain sizes can be probed in NMR using ${ }^{1} \mathrm{H}$ proton spin diffusion, during a "mixing" time $t_{\mathrm{m}}$ on the $\mathrm{ms}$ to 0.5 -s time scale. During the evolution period of a two-dimensional experiment, the magnetization of protons in one phase (A) is modulated by its characteristic chemical-shift frequency $\omega_{\mathrm{A}}$. If during $\mathrm{t}_{\mathrm{m}}$ the magnetization 
diffuses to protons in a different phase (B), it will be detected with frequency $\omega_{\mathrm{B}}$; thus, domain proximity on the spin diffusion length scale $\left(0.5-30 \mathrm{~nm}\right.$, depending on $\left.\mathrm{t}_{\mathrm{m}}\right)$ results in an $\left(\omega_{\mathrm{A}}, \omega_{\mathrm{B}}\right)$ cross peak in the two-dimensional spectrum. For small domain sizes, the equilibration is a fast process, while for large domains it is relatively slow. In the present case, phosphate protons are only a small percentage of the protons in the sample and therefore difficult to detect. Hence, we detect them indirectly, with excellent selectivity, in terms of ${ }^{31} \mathrm{P}$ spins to which these protons cross-polarize.

The experiment with ${ }^{1} \mathrm{H}$ evolution flanked by excitation and $\mathrm{z}$-storage $90^{\circ}$ pulses, spin diffusion time $t_{\mathrm{m}}$, read-out pulse, cross polarization to ${ }^{31} \mathrm{P}$, and ${ }^{31} \mathrm{P}$ detection effectively is a 2D WISE experiment [17]. At short $t_{m}$, the slice along the ${ }^{1} \mathrm{H}$ dimension will reflect only the phosphate protons near the detected ${ }^{31} \mathrm{P}$ spins; at longer $\mathrm{t}_{\mathrm{m}}$ times, if there is spin diffusion contact between the phosphate protons and the protons from the surrounding polymer matrix, the ${ }^{1} \mathrm{H}$ lineshape will change to that of the polymer protons. This approach was previously demonstrated in polymer-clay nanocomposites using ${ }^{1} \mathrm{H}-{ }^{29} \mathrm{Si}$ WISE NMR by Hou et al [18]

\subsection{Results and Discussion}

The PDEAEM pentablock copolymer was synthesized by ATRP technique. The molecular weight of the polymer was characterized by GPC. The molecular weight of the copolymer was found to be 30,633 with a narrow molecular weight distribution $(\mathrm{Mw} / \mathrm{Mn}<$ 1.33). Figure 4.2 shows the ${ }^{1}$ HNMR characterization of the polymer. 


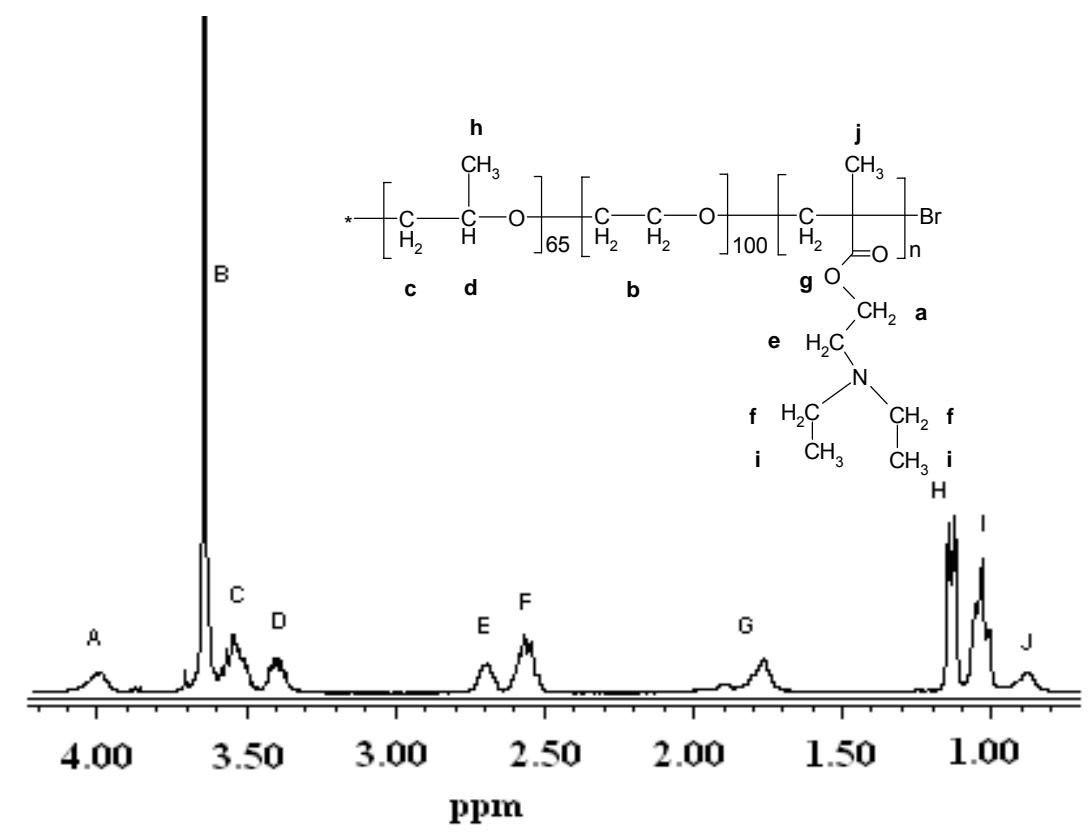

Figure 4.2. ${ }^{1} \mathrm{HNMR}$ of DEAEM pentablock in $\mathrm{CDCl}_{3}$

The tertiary amines of the PDEAEM groups are protonated and hydrophilic below the $\mathrm{pK}_{\mathrm{a}}$ (7.5) of the monomer[15]. Figure 4.3 shows the phase diagram of this pentablock copolymer.

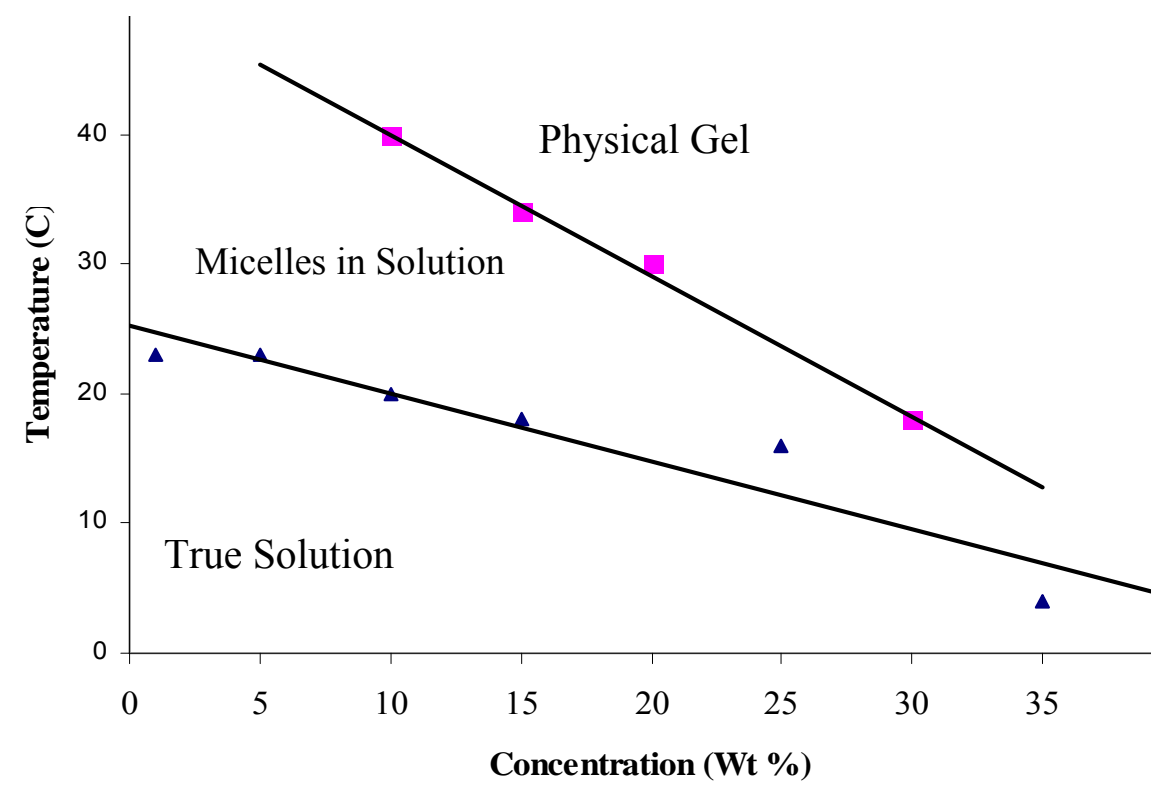

Figure 4.3. Phase diagram of the DEAEM pentablock copolymer [data obtained by Determan et al [15]] 
The polymer exhibits thermoreversible properties due to the lower critical solution temperature (LCST) of the central poly(propylene oxide) (PPO) block. The pentablock copolymer micellar solutions form a lyotropic liquid crystalline phase that results in a transparent hydrogel above a critical gel concentration (CGC).

First, the inorganic fractions in the nanocomposite samples were tested with TGA, by burning off the polymer totally at $400^{\circ} \mathrm{C}$. TGA experiments indicated that the inorganic percentage of $\mathrm{pH} 1.0$ pentablock nanocomposite samples to be around $15 \mathrm{wt} \%$ while the $\mathrm{pH}$ 3.0 samples only had $6.5 \mathrm{wt} \%$. The mass percentage was based on the dried gel samples at $250^{\circ} \mathrm{C}$. Transmission electron microscopy of the pentablock gel prepared with the $\mathrm{pH} 1.0$ calcium phosphate solution clearly indicated aggregates of spheres.

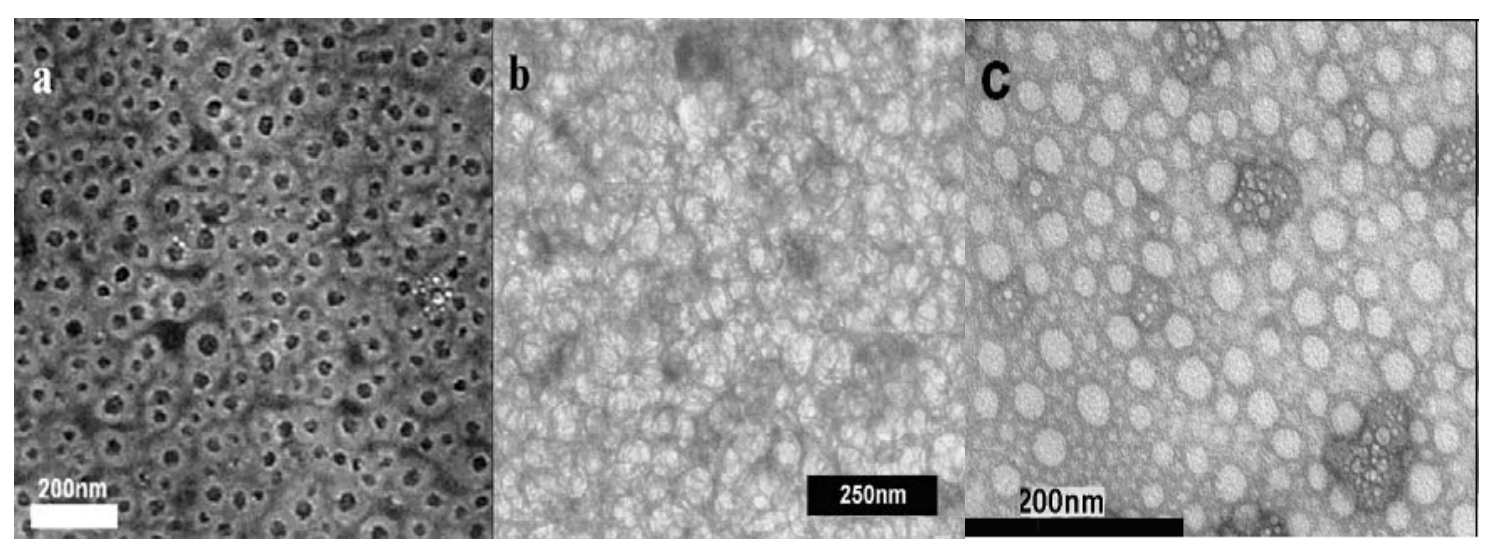

Figure 4.4. Cryo-TEM Micrographs of a) $3 \mathrm{wt} \%$ Pentablock Micelle Solution [data obtained by Determan et al [15]] and b) $30 \mathrm{wt} \%$ Pentablock-pH 1.0 Calcium Phosphate Gel c) $3 \mathrm{wt} \%$ Pentablock-calcium phosphate micelles at $\mathrm{pH} 3.0$ [Data obtained by Enlow]

The cryo-frozen gel appeared as a concentrated matrix of $\sim 60 \mathrm{~nm}$ diameter spheres (Figure 4.4b). That observation is consistent with the TEM analysis done by Determan et al [8] who observed $60-90 \mathrm{~nm}$ diameter total diameter spheres in a $3 \mathrm{wt} \%$ pentablock micelle solution (Figure 4.4a- [15]). As seen in figure 4.4c, micelles guide precipitation of the 
darker-appearing more electron dense brushite / monetite coating after 24 hours of aging. However, many uncoated micelles still remain in solution. The EDS analysis showed strong calcium and phosphate signal may be attributed to the amorphous calcium and phosphate ions in solution, or due to the precipitate of an inorganic calcium phosphate phase in solution.

SAXS analysis was performed to investigate the ordered superstructure of the polymer-based nanocomposites. As shown in Fig (4.5), the self-assembled Pluronic ${ }^{\circledR}$ copolymer gel with and without calcium phosphate nanocomposites both at $\mathrm{pH} 1.0$ and 3.0 has distinct diffraction peaks with $\mathrm{Q}^{\mathrm{Q}} \mathrm{Q}^{*}\left(\mathrm{Q}^{*}\right.$ is the first order peak position) of $\sqrt{3}: \sqrt{4}: \sqrt{8}: \sqrt{11}: \sqrt{12}$, indicating that the system exhibits a face-centered cubic (FCC) structure. The Pluronic ${ }^{\circledR}$ with calcium phosphate at $\mathrm{pH} 1.0$ has additional higher order peaks, which implies that the system is better ordered. In addition, the Pluronic ${ }^{\circledR}$ gel with and without inorganic phase at $\mathrm{pH} 3.0$ showed similar inter-particle distances, $\mathrm{D}\left(D=\sqrt{3} d_{111}\right.$, where $\left.d_{111}=\frac{2 \pi}{Q^{*}}\right)$. At $\mathrm{pH} 1.0$, however, $\mathrm{D}$ increases from 15.5 to $17.1 \mathrm{~nm}$ with the addition of calcium phosphate. This may be attributed to the different level of calcium phosphate precipitation over polymer micelles at different $\mathrm{pH}$ values. At $\mathrm{pH} 3.0$, the precipitation rate of calcium phosphate is low, the weight fraction of the inorganic phase is $7 \%$ as compared to $11 \%$ at $\mathrm{pH} 1.0$ as seen from TGA experiments (data not shown) and only few micelles were coated with calcium phosphate, as seen in the TEM images. Due to the large number of micelles without inorganic coatings, the micelles coated with the calcium phosphate do not contribute to the scattering. Hence, the D value remains similar in both Pluronic ${ }^{\circledR}$ gels with and without the inorganic phase at $\mathrm{pH} 3.0$. At $\mathrm{pH} 1.0$, the higher ionic concentration expedites the precipitation of calcium phosphate, and more micelles are coated with the 
inorganic phase. Thus, the micelle size increases due to the inorganic phase precipitation on the Pluronic ${ }^{\circledR}$, causing an increase in D. This result is in agreement with the TEM data.

As for the pentablock copolymer gel nanocomposites in Figure 4.6, few SAXS peaks are observed. Besides the peaks are broader than those for the Pluronic ${ }^{\circledR}$ gels, which means that the system is relatively poorly ordered. However, comparing the SAXS patterns of the Pluronic ${ }^{\circledR}$ and pentablock gels, the trends of the profiles are fairly consistent. Therefore, the morphology of pentablock gels with and without calcium phosphate may also exhibit FCC structure, and any broad peak has contributions from several peaks.

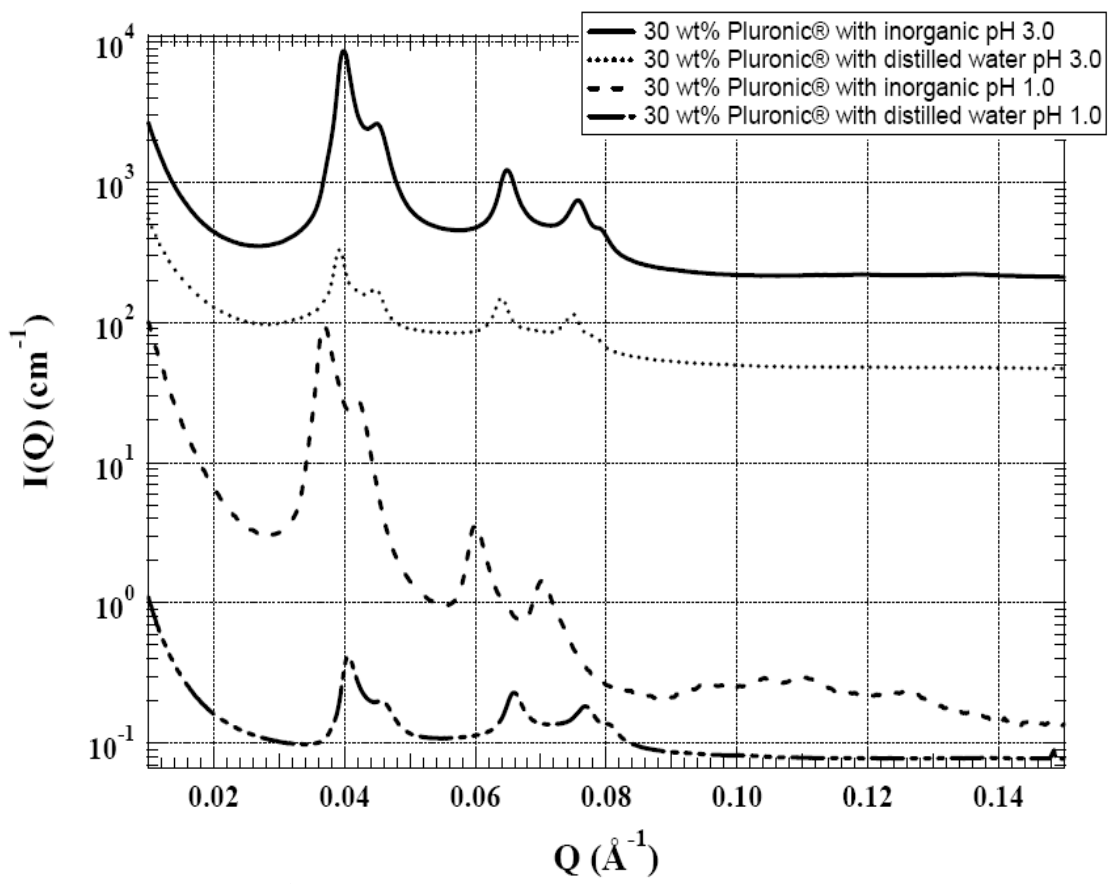

Figure 4.5. SAXS of Pluronic ${ }^{\circledR}$ F127 gels with and without calcium phosphate. Scattering patterns have been shifted vertically for clarity.

For the pentablock copolymer gel with calcium phosphate at $\mathrm{pH} 3.0$, no distinct higher order peaks were observed. This is presumably due to the formation of larger calcium phosphate particles in the interstitial spaces that disrupt the ordered pentablock copolymer packing structure. Compared to the D of the pure pentablock copolymer gel, there is a 
dramatic increase in D when the pentablock copolymer gels are coated with calcium phosphate at $\mathrm{pH}$ 1.0. This is consistent with the results seen with Pluronic ${ }^{\circledR}$ gels with calcium phosphate at $\mathrm{pH} 1.0$.

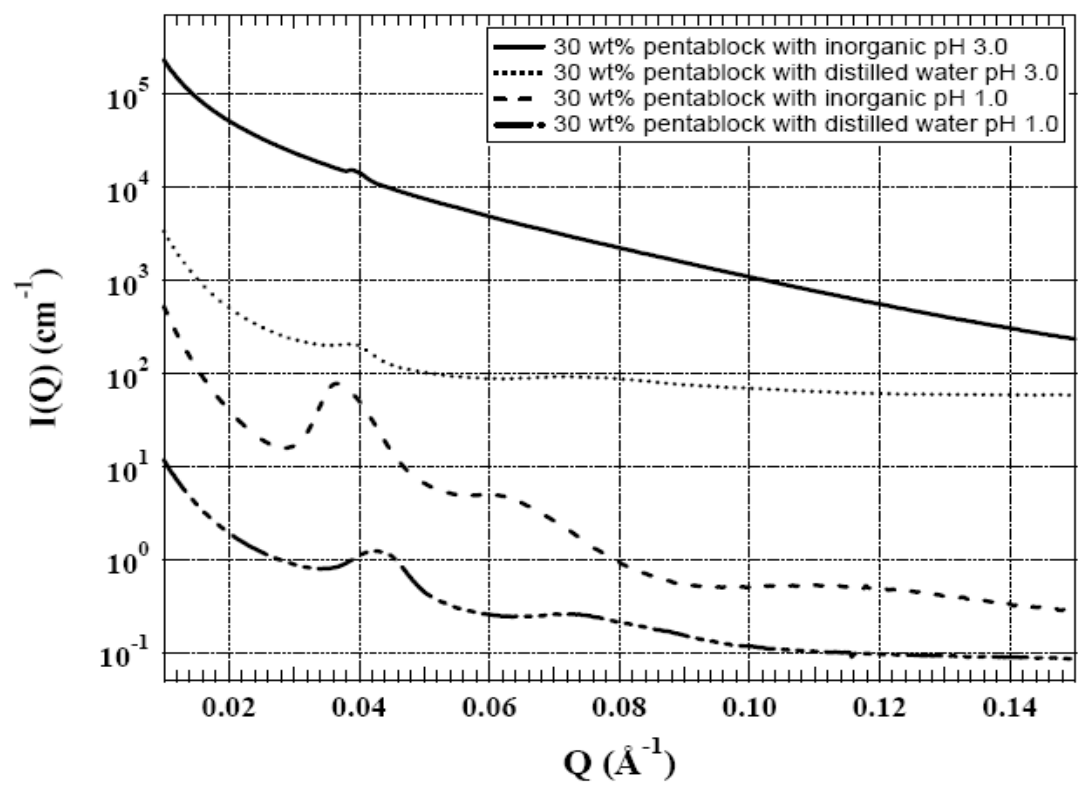

Figure 4.6. SAXS of pentablock copolymer gels with and without calcium phosphate. Scattering patterns have been shifted vertically for clarity.

XRD measurements revealed that the precipitates formed in gels at $\mathrm{pH} 3.0$ are similar to cave brushite, a natural form of brushite found in caves, which mimics the natural process well. Further, using the Sherrer equation, the brushite crystal sizes were found to be around $30 \mathrm{~nm}$ or bigger, from the widths of the Bragg peaks. On the other hand, at low $\mathrm{pH}(\mathrm{pH} \sim$ 1.0), synthetic brushite was observed. This is believed to be due to the higher driving force due to higher concentrated solution and hence less controlled precipitation of inorganic phase.

The composition of the phosphate in the inorganic precipitation was assessed by PNMR as well. In the case of Pluronic ${ }^{\circledR}$, the NMR resonance indicated a monohydrogen phosphate (CaHPO4) as found in brushite/ monetite. In the case of pentablock templated 
precipitation calcium dihydrogen phosphate seems to be the dominated phase. Phosphatepolymer proximity was studied using the popular H-P WISE with $\mathrm{H}$ spin diffusion, which is used to characterize the proximity of the inorganic components to the organic matrix. From the WISE spectra of Pluronic ${ }^{\circledR}$-nanocomposite (Figure 4.7), the $\mathrm{H}$ spin diffusion from the polymer to the brushite protons occurs less than $50 \mathrm{~ms}$ (spin diffusion time). For protonated species the spin diffusion coefficient is taken as $<0.5 \mathrm{~nm}^{\wedge} 2 \mathrm{~m}-1$, the domain sizes were found to be less than $20 \mathrm{~nm}$. On the contrary, there is hardly any evidence of spin diffusion contact was observed for pentablock composite, indicating larger particles $>20 \mathrm{~nm}$ in diameter, in which most of the protons are far way from the polymer matrix. This is in agreement with XRD crystallite size of $>30 \mathrm{~nm}$, estimated from FWHM of the Bragg peaks of the composite sample.

a)

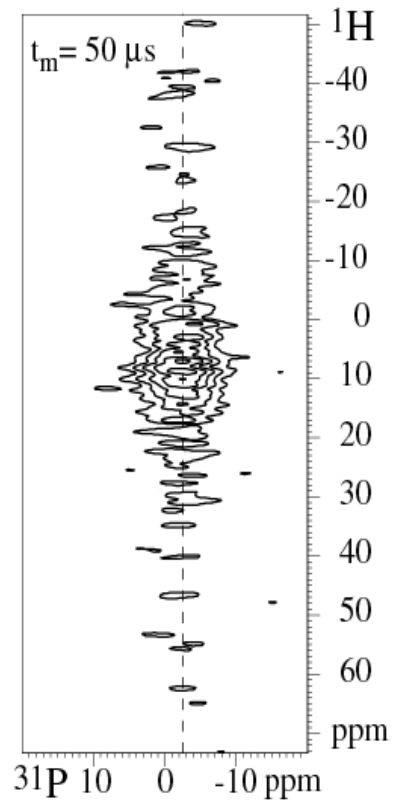

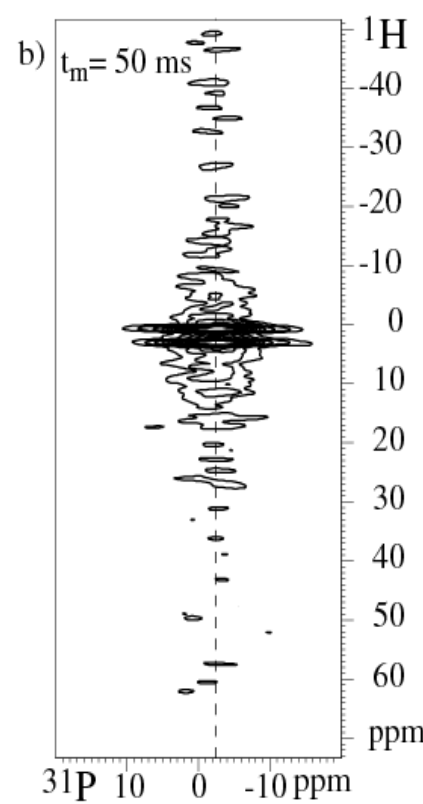

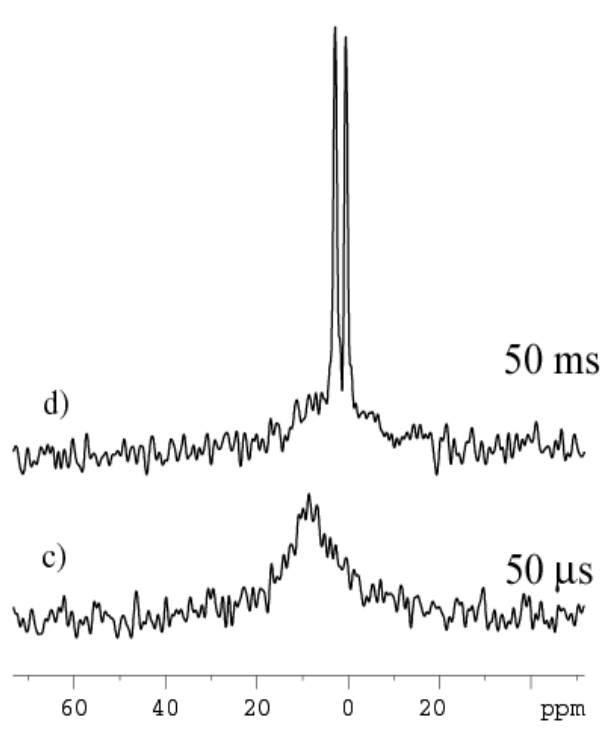

Figure 4.7. ${ }^{1} \mathrm{H}\left\{{ }^{31} \mathrm{P}\right\} 2 \mathrm{D}$ WISE at $6.5 \mathrm{kHz}$ MAS of the Pluronic ${ }^{\circledR} \mathrm{F} 127$-phosphate composite. (a) and (b) are 2D contour plots of spectra recorded after 0.05 and $50 \mathrm{~ms}$ of spin diffusion, respectively. Figures (c) and (d) are slices along the ${ }^{1} \mathrm{H}$ dimension extracted at $1.6 \mathrm{ppm}$ in the ${ }^{31} \mathrm{P}$ dimension from the spectra in (a) and (b), respectively [Data obtained by Rawal et al] 


\subsection{Conclusions}

The formation of macroscale polymer-inorganic nanocomposites with ordered microand nanostructures completely by self assembly was demonstrated.With a careful copolymer design and synthesis and the use of gel-forming self-assembling copolymers, it is possible to synthesize macro-sized ordered networks of nanocomposites. This bottom-up approach to materials design compared to previous works on mineralizing the surface of bulk polymers[46] or forming sub-micron aggregates of organic-inorganic nanocomposites[7] is a novel approach in this respect. It has been demonstrated that a nanocrystalline calcium phosphate phase can be formed from aqueous solutions onto self-assembling copolymer micelles and gels similar to the mineralization of collagen in bone formation.

Since the inorganic fractions in the samples that can be achieved are very low $(10 \%)$ compared to nature $(70 \%)$, currently we are investigating on improving the inorganic precipitation by enhancing the inorganic-organic interaction. Polymer templates with ionic groups may be a good approach in this respect and the polymer-inorganic interactions can potentially be varied by changing the ionic character of the polymer templates for better inorganic precipitation on the organic templates. The following chapters describe the ongoing work on biomineralization using ionic polymer templates to increase the inorganic fraction of the nanocomposite while maintaining the polymer order and ensuring templated inorganic nanocrystal formation. Further insight into the nucleation and growth of calcium phosphate on polymer surfaces will lead to a better understanding of this biomimetic approach leading to future recreation of similar materials.

\subsection{Acknowledgements}


This work was supported by the U.S. Department of Energy under contract number DE-AC02-07CH11358 for Ames Laboratory, Iowa State University. This work benefited from the use of APS at Argonne National Lab, funded by the U.S. DOE, office of science, office of basic science under contract no DE-AC02-06CH11357. The authors also wish to thank Dr. Mike Determan and Y. Yusufoglu, for their help with the project.

\subsection{References}

1. Rao, P.R., Biomimetics. Sadhana, 2003. 28(3\&4): p. 657-676.

2. Ikkala, O. and G. ten Brinke (2004). "Hierarchical self-assembly in polymeric complexes: towards functional materials." Chemical Communications (Cambridge, United Kingdom)(19): 2131-2137.

3. Zhang, S. (2003). "Fabrication of novel biomaterials through molecular selfassembly." Nature Biotechnology 21(10): 1171-1178.

4. Li, C. and D. L. Kaplan (2003). "Biomimetic composites via molecular scale selfassembly and biomineralization." Current Opinion in Solid State \& Materials Science 7(4-5): 265-271.

5. Muller, F.A., et al. Biomimetic Apatite Formation on Chemically Modified Cellulose Templates. in The Annual Meeting of the International Society for Ceramics in Medicine, Nov 6-9 2003. 2004. Porto, Portugal: Trans Tech Publications Ltd.

6. Song, J., E. Saiz, and C.R. Bertozzi, A new approach to mineralization of biocompatible hydrogel scaffolds: An efficient process toward 3-dimensional bonelike composites. Journal of the American Chemical Society, 2003. 125(5): p. 1236-1243.

7. Song, J., E. Saiz, and C.R. Bertozzi, Preparation of pHEMA-CP composites with high interfacial adhesion via template-driven mineralization. Journal of the European Ceramic Society, 2003. 23(15): p. 2905-2919.

8. Rusu, V.M., et al., Size-controlled hydroxyapatite nanoparticles as self-organized organic-inorganic composite materials. Biomaterials, 2005. 26(26): p. 5414-5426

9. Tanahashi, M. and T. Matsuda (1997). "Surface functional group dependence on apatite formation on self-assembled monolayers in a simulated body fluid." Journal of Biomedical Materials Research 34(3): 305-315.

10. Zhao, Y. F. and J. Ma (2005). "Triblock co-polymer templating synthesis of mesostructured hydroxyapatite." Microporous and Mesoporous Materials 87(2): 110117.

11. Determan, M. D., L. Guo, et al. (2006). "Supramolecular Self-Assembly of Multiblock Copolymers in Aqueous Solution." Langmuir 22(4): 1469-1473.

12. Wanka, G., H. Hoffmann, et al. (1990). "The aggregation behavior of poly(oxyethylene)-poly(oxypropylene)-poly(oxyethylene) block copolymers in aqueous solution." Colloid and Polymer Science 268(2): 101-17.

13. Wanka, G., H. Hoffmann, et al. (1994). "Phase Diagrams and Aggregation Behavior of Poly(oxyethylene)-Poly(oxypropylene)-Poly(oxyethylene) Triblock 
Copolymers in Aqueous Solutions." Macromolecules 27(15): 4145-9.

14. Pozzo, D. C. and L. M. Walker (2007). "Small-angle neutron scattering of silica nanoparticles templated in PEO-PPO-PEO cubic crystals." Colloids and Surfaces, A: Physicochemical and Engineering Aspects 294(1-3): 117-129.

15. Determan, M. D., J. P. Cox, et al. (2005). "Synthesis and characterization of temperature and pH-responsive pentablock copolymers." Polymer 46(18): 69336946.

16. Chen, Q., S.S. Hou, and K. Schmidt-Rohr, A simple scheme for probehead background suppression in one-pulse $<$ sup $>1</$ sup $>$ H NMR. Solid State Nuclear Magnetic Resonance, 2004. 26(1): p. 11-15.

17. Schmidt-Rohr, K., J. Clauss, and H.W. Spiess, Correlation of structure, mobility, and morphological information in heterogeneous polymer materials by twodimensional wideline-separation NMR spectroscopy. Macromolecules, 1992. 25(12): p. 3273-3277.

18. Hou, S.S., F.L. Beyer, and K. Schmidt-Rohr, High-sensitivity multinuclear NMR spectroscopy of a smectite clay and of clay-intercalated polymer. Solid State Nuclear Magnetic Resonance, 2002. 22(2-3): p. 110-127.

19. Enlow, D., et al., Synthesis and characterization of self-assembled block copolymer templated calcium phosphate nanocomposite gels. Journal of Materials Chemistry, 2007. 17(16): p. 1570-1578. 


\section{CHAPTER 5. SYNTHESIS AND CHARACTERIZATION OF IONIC BLOCK COPOLYMER TEMPLATED CALCIUM PHOSPHATE NANOCOMPOSITES}

(This chapter is based on collaborative work accepted by Chemistry of Materials and has been modified to focus mainly on my contributions to this work)

M. Kanapathipillai ${ }^{\mathrm{a}, 1}$, Y.Yusufoglu ${ }^{\mathrm{c}, 1}$, A. Rawal ${ }^{\mathrm{b}}$, Y.-Y. Hu ${ }^{\mathrm{b}}$, C.-T. Lo ${ }^{\mathrm{d}}$, P. Thiyagarajan ${ }^{\mathrm{e}}$, Y.E. Kalay ${ }^{c}$, M. Akinc ${ }^{c}$, S. Mallapragada ${ }^{\mathrm{a}}$, K. Schmidt-Rohr, ${ }^{\mathrm{b}, *}$

\subsection{Abstract}

Thermo reversible anionic and zwitterionic pentablock copolymers were used as three-dimensional templates for efficient precipitation, and for controlling the size and the ordered structural arrangement of calcium phosphate nanocrystals. Calcium and phosphate ions were dispersed in the block-copolymer micellar liquid phase at low temperatures and inorganic nanocrystals were believed to be formed by the ionic interactions at the polymerinorganic interface as they were driven into the interstitial cavities of the polymer micelle structures by aging at room temperature. Small Angle X-ray and Neutron Scattering (SAXS and SANS) along with X-ray Diffraction (XRD), Nuclear Magnetic Resonance (NMR), Theromogravimetric Analysis (TGA) and Transmission Electron Microscope (TEM) were used to characterize the self-assembled polymer-inorganic nanocomposites. SANS data indicate that the addition of the inorganic phase enhances the polymer structural order, making it more compact. This is in good agreement with rheological measurements where the polymer-inorganic nanocomposite gels exhibited higher moduli values compared to the polymer gels alone. Solid state NMR studies show that the average inorganic particles 
formed are of nanometer length scale and they are in good contact with the polymer phase, which is also observed from TEM analysis. SAXS data revealed that the polymer template significant fraction of the calcium phosphate. Further, according to TGA measurements, the inorganic percentage in the dried nanocomposite gel appears to be around $30 \%$. Nanoscale calcium phosphate crystals with significant inorganic fractions were achieved using selfassembling ionic block copolymer templates through a bottom-up approach.

\footnotetext{
${ }^{a}$ Ames Laboratory and Department of Chemical Engineering, Iowa State University

${ }^{\mathrm{b}}$ Ames Laboratory and Department of Chemistry, Iowa State University

${ }^{c}$ Ames Laboratory and Department of Materials Engineering, Iowa State University

dAdvanced Photon Source, Argonne National Laboratory

Intense Pulsed Neutron Source, Argonne National Laboratory

${ }^{1}$ Both authors contributed equally to this research

* Corresponding author: Klaus Schmidt-Rohr, Dept. of Chemistry, Iowa State University, Ames, IA 50011. srohr@iastate.edu 515-294-6105
}

\subsection{Introduction}

In most examples of successful templating of polymer-ceramic composites, the polymer-inorganic phase interaction is limited to the surface of the polymer and restricts the systematic study of the effects that variables such as ionic interaction, relative size and concentration of polymer have on templating and nanocrystal formation ${ }^{1-4}$. One method to create nanocomposites and also to expand its applicability to the creation of threedimensional macro-scale structures is the dispersion of already synthesized particles or ionic solutions into the structured matrix ${ }^{4,5}$. But nanoparticle aggregation, high viscosity of the nanostructured materials and incompatibility of the particle surface and ionic solutions with the polymers limit the use of these types of templating methods ${ }^{5}$. We have demonstrated that these problems can be circumvented by the use of self-assembling thermo-reversible 
block copolymer gels that can facilitate a bottom-up approach for inorganic nanocomposite synthesis ${ }^{6}$. Although successful templating was achieved by this approach, the percentage of inorganic precipitate in the final nanocomposite was low ( $\sim 15 \mathrm{wt} \%)$, compared to about $70 \mathrm{wt} \%$ seen in nature. ${ }^{7}$

In the bone formation process, collagen fibrils are formed by self-assembly of collagen triple helices and hydroxyapatite (HAp) crystals grow within these fibrils ${ }^{8}$. During the HAp crystal growth, the acidic macromolecules such as glycoproteins that are attached to the collagen scaffold play important templating roles. Glycoproteins are covalently linked to polysaccharide side chains that often contain sulfate and carboxylate residues, and these functional groups serve as binding sites for $\mathrm{Ca}^{2+}$ ions ${ }^{9}$. To mimic natural nanocomposites better, the ionic interactions between the organic-inorganic interfaces need to be enhanced. Tanahashi et al. ${ }^{10}$ have shown using self-assembled monolayers that anionic polymers template better compared to cationic or non-ionic polar polymers and further, ionic blocks such as carboxylate groups or phosphobetaines are ideal for calcium phosphate precipitation. Other studies have also indicated that carboxyl groups not only provide a site for heterogeneous nucleation of apatite, but also contribute to a tight adhesion of the apatite layer to polymer films. ${ }^{11}$ Moreover, Spanos and coworkers ${ }^{12}$ synthesized a novel composite with $9 \%$ HAp and $91 \%$ polymer by weight made of a biocompatible synthetic polymer (sulphonated polysulfone) and HAp by the precipitation of calcium phosphate phase in aqueous suspensions of the polymer particles.

Theoretical results using molecular dynamics simulations ${ }^{13}$ have established general conditions for successful nanotemplating methods. It has been shown, for instance, that polymers with end-group functionalized blocks with a specific affinity for the inorganic 
components provide a successful templating strategy ${ }^{14}$. In this respect, block copolymers containing blocks of sulfobetaines, which are analogues to phosphobetaines, and blocks of acrylic acids, which contain carboxylate groups, are good choices for polymer templates in biomimetic mineralization. Further, it is believed that the interactions of acrylic-acid carboxylate groups with $\mathrm{Ca}^{2+}$ ions mimic those of the carboxylate groups in bone ${ }^{15}$. Hence, many mineralization studies have focused on poly(acrylic acid) based templates for surface mineralization studies ${ }^{15-17}$. However, to enable hierarchical self-assembly of nanocomposites, copolymers that can self-assemble in three dimensions at multiple length scales in the presence of the inorganic phase need to be synthesized and investigated.

As hierarchically assembling copolymer templates, we have synthesized pentablock copolymers consisting of central triblock $\mathrm{PEO}_{100}-\mathrm{PPO}_{65}-\mathrm{PEO}_{100}$ Pluronic ${ }^{\circledR}$ F127 copolymers modified with zwitterionic or anionic terminal blocks. The zwitterionic pentablock copolymers contain polysulfobetaine side chains, with an isoelectric point (iep) value of about 6.3. This polymer is zwitterionic over a wide range of $\mathrm{pH} \approx 2-11$. The anionic pentablock copolymer, on the other hand, contains acrylic acid blocks and imparts $\mathrm{pH}$ sensitivity above $\mathrm{pH} \sim 6$. In addition to the ionic nature, the thermo-reversible ordering behavior of these polymers and their water solubility make them ideal templating materials for formation of nanocomposites. Here we study the influence of ionic moieties of the polymer template on the formation of inorganic nanocomposites by self-assembly of thermoreversible ionic block-copolymer gel structures, a bottom-up approach that has been reported by us with cationic and Pluronic ${ }^{\circledR}$ block-copolymer gels previously ${ }^{6}$. Further, along with other characterization methods, we present nuclear magnetic resonance (NMR), scattering, and electron microscopy results that support the structural picture of the polymer-inorganic 
nanocomposite superstructure.

\subsection{Materials and Methods}

All materials used were obtained from either Sigma Aldrich or Fisher Scientific and were of laboratory grade and purity.

Pentablock copolymer synthesis. Ionic pentablock copolymers were synthesized by atom transfer radical polymerization (ATRP). First, a cationic pentablock copolymer with poly(diethylaminoethylmethacrylate) (PDEAEM) side chains was synthesized by ATRP as described earlier. ${ }^{18}$ This was followed by betanization of the tertiary amine groups of the side chains with propanesultone, to form the zwitterionic pentablock copolymer (PentaPZ). The synthesis procedure is shown in scheme 1 . In a typical reaction, pentablock polymer (6 g, $0.03 \mathrm{mmol})$, and 1, 3-propanesultone (30 g, $0.3 \mathrm{mmol})$ were dissolved in dry THF. The mixture was then reacted at $40^{\circ} \mathrm{C}$ under argon for 2 days with continuous stirring. The reaction mixture was then precipitated with diethyl ether and vacuum dried.

The synthesis scheme of the anionic pentablock copolymer (PentaPAA) is shown in Scheme 2. For its synthesis, first the Pluronic ${ }^{\circledR}$ macroinitiator was prepared as reported previously ${ }^{18}$. Difunctional Pluronic ${ }^{\circledR}$ macroinitiator (8 g, $0.06 \mathrm{mmol}$ ), copper bromide $(0.1885 \mathrm{~g}, 0.06 \mathrm{mmol})$, and toluene $(50 \mathrm{~mL})$, were added to a flask, and the solution was mixed well by stirring for 15 minutes. A small amount of copper was added to the reaction to enhance the stability of the $\mathrm{Cu}(\mathrm{I}) / \mathrm{Cu}(\mathrm{II})$ equilibrium. The flask was purged with Argon for 5 min. Tert-butyl acrylate $(2.8 \mathrm{~g}, 2.1 \mathrm{mmol})$ and $\mathrm{N}$-ppm ligand $(0.37 \mathrm{~g}, 0.12 \mathrm{mmol})$ were added using a syringe. The mixture was freeze-pumped/thawed thrice. The reaction was then carried out at $65^{\circ} \mathrm{C}$ in an oil bath overnight. The reaction mixture was then diluted in methylene chloride and passed through a short alumina column to remove the copper catalyst 
and was rotary evaporated. The remaining mixture was re-precipitated in n-heptane and dried under vacuum. Finally, the synthesized acrylate polymer was hydrolyzed with THF and trifluoro acetic acid (TFA) $(20 \% \mathrm{v} / \mathrm{v})$ mixture by stirring overnight. The mixture was precipitated in n-heptane and the acrylic acid polymer was isolated after vacuum drying.

\section{SCHEMES}

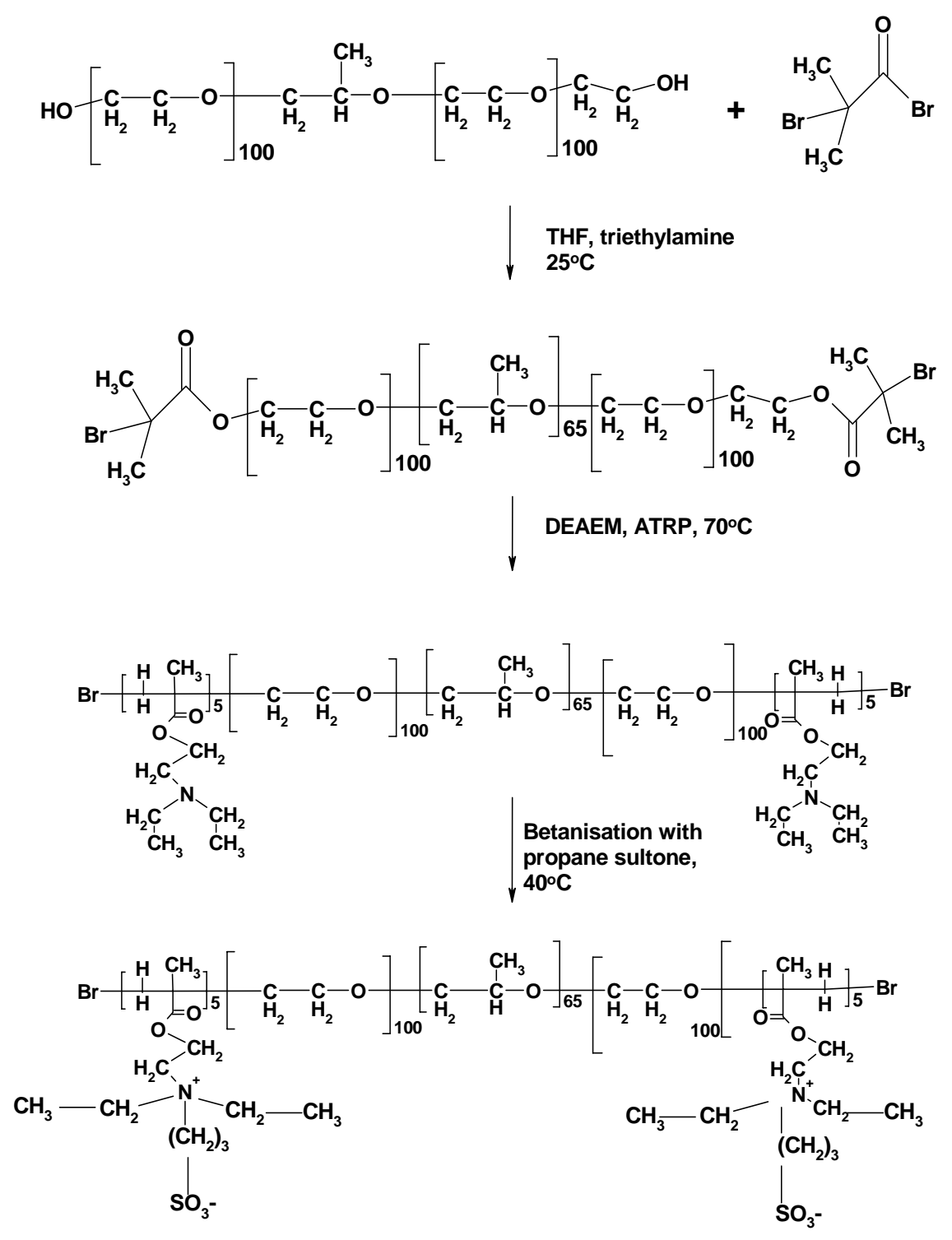

Scheme 1. Synthesis of the zwitterionic pentablock copolymer ('PentaPZ'). 

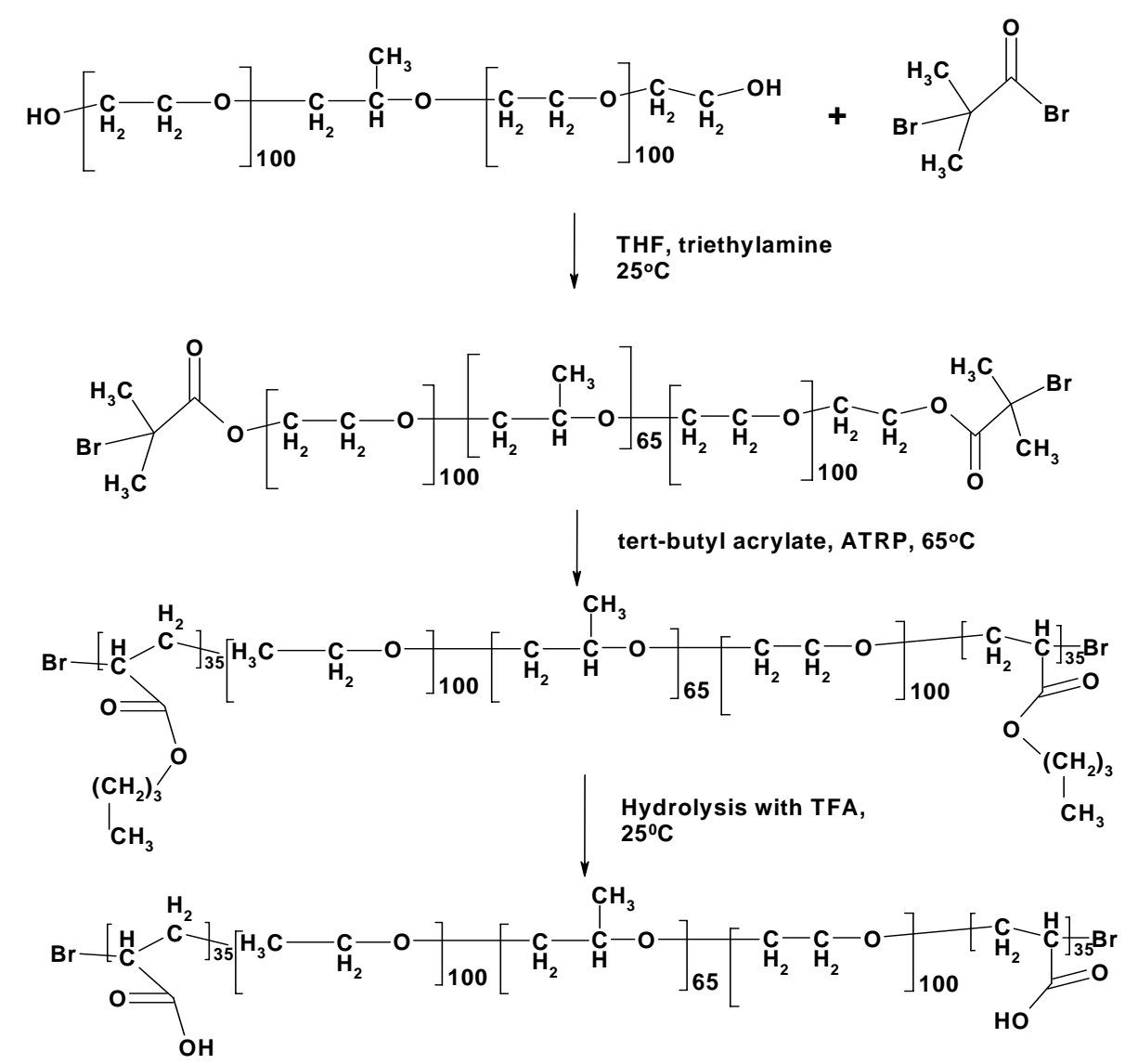

Scheme 2. Synthesis of the anionic pentablock copolymer ('PentaPAA').

Pentablock copolymer characterization. Solution NMR measurements were performed on a Varian VXR400 spectrometer, GPC measurements using PLgel columns from Polymer Laboratories, to determine the composition and molecular weights, respectively, of the block copolymers. Simple tube inversion was used to find the gelation temperature. Titration measurements were carried out using a Corning $313 \mathrm{pH} /$ temperature meter at room temperature to find the $\mathrm{pK}_{\mathrm{a}}$ and iep values, and differential scanning calorimetry (DSC) in a Thermal Analysis DSC instrument Q 20 was used to find the critical micellization temperatures of the copolymer solutions. The micelle formation in water is an endothermic first-order transition due to the enthalpy of dehydration of the hydrophobic block ${ }^{19}$. 
Measurements were carried out using a Thermal Analysis DSC instrument Q 20 equipped with an auto sampler. Samples with masses ranging from 5 to $20 \mathrm{mg}$ were heated from -5 to $50^{\circ} \mathrm{C}$ at a rate of $5^{\circ} \mathrm{C} / \mathrm{min}$. The data were corrected by sample weight normalization and subtraction of the linear baseline.

Titration measurements were carried out to obtain the degree of ionization. Potentiometric titrations were carried out using a Corning $313 \mathrm{pH} /$ temperature meter at room temperature. Samples were prepared by dissolving $500 \mathrm{mg}$ of polymer in $50 \mathrm{~mL}$ nanopure water. In the case of Penta-PZ copolymers, after the complete dissolution of the polymer in water, the solution was titrated using $1.0 \mathrm{M} \mathrm{NaOH}$. A pH vs $\left[\mathrm{OH}^{-}\right]$concentration plot was obtained and the iep value was obtained from the inflection point. For the anionic pentablock copolymer, the solution $\mathrm{pH}$ was first raised to $\mathrm{pH} 12$ by adding $1.0 \mathrm{M} \mathrm{NaOH}$ and then back titrated using 1.0 M HCl. The degree of ionization, $\alpha$, was calculated from the ratio of the net concentration of $\mathrm{H}^{+}$ions $\left(\mathrm{C}_{\mathrm{H}^{-}} \mathrm{C}_{\mathrm{OH}}\right)$ and $\mathrm{COOH}$ groups $\left(\mathrm{C}_{\mathrm{A}}\right)$ in solution, $\alpha=\frac{\left(C_{H}-C_{O H}\right)}{C_{A}}{ }^{20}$

Pentablock-calcium phosphate nanocomposite synthesis. The calcium phosphate-polymer nanocomposite gel was synthesized as reported [Kanapthipillai et al., Chemistry of Materials]. Briefly, 2.9 g PentaPAA copolymer was mixed with $5.0 \mathrm{~mL} 4.0 \mathrm{M} \mathrm{Ca}\left(\mathrm{NO}_{3}\right)_{2}$ in $25 \mathrm{mM}$ tris- $\mathrm{HCl}$ solution and was kept refrigerated overnight (at $\sim 1{ }^{\circ} \mathrm{C}$ ). Then, $3.0 \mathrm{~mL} 4.0 \mathrm{M}$ $\left(\mathrm{NH}_{4}\right)_{2} \mathrm{HPO}_{4}$ in $25 \mathrm{mM}$ tris- $\mathrm{HCl}$ solution was added. The mixture was allowed to equilibrate for 1.5 days in the refrigerator. Then the sample was aged at room temperature for two days resulting in a hydrogel sample. The polymer content is around $27 \%$ by weight and the $\mathrm{pH} \approx 5$ (this sample is referred to as PentaPAA27-5). A similar procedure was followed to obtain PentaPAA36-5, where the numbers 36 and 5 again refer to $\mathrm{wt} \%$ of the polymer and $\mathrm{pH}$ of the 
gel, respectively. In the case of the zwitterionic PentaPZ copolymer, an organic-inorganic hydrogel with a polymer concentration of approximately 46 wt $\%$ (PentaPZ46-5) was produced in a similar fashion.

Characterization Methods. Various experiments were performed to study the structural features of the polymer-based nanocomposite. SAXS and SANS can determine the ordering in the gels; due to the large electron-density contrast between the organic and inorganic components, SAXS has a higher sensitivity on the structure of the inorganic phase while SANS probes mainly the structure of the polymer phase by utilizing the large contrast between ${ }^{1} \mathrm{H}$ and ${ }^{2} \mathrm{H}$ in the polymer and $\mathrm{D}_{2} \mathrm{O}$, respectively ${ }^{5}$. SAXS measurements were performed at the 12-ID beam line at the Advanced Photon Source in Argonne National Laboratory, while SANS measurements were carried out using the time-of-flight small-angle neutron diffractometer (SAND) at IPNS at Argonne National Laboratory. Solid-state NMR can prove nanocomposite formation and provide information regarding the size of inorganic nanoparticles as well as their composition. Solid-state NMR experiments were run using a Bruker DSX400 spectrometer (Bruker-Biospin, Rheinstetten, Germany) at $400 \mathrm{MHz}$ for ${ }^{1} \mathrm{H}$ and $162 \mathrm{MHz}$ for ${ }^{31} \mathrm{P}$. Rheological experiments were carried out using an ARES rheometer (TA Instruments, New Castle, Delaware). TEM analysis illustrates the size of the precipitated nanosize calcium phosphate particles and helps characterize them. XRD was used to identify the crystalline phases of the inorganic component. TGA measurements yielded the percentage of inorganic material in the nanocomposite.

SAXS measurements were performed with $1 \mathrm{~mm}$ thick gel samples. The gels were sandwiched between 25 micron thick Kapton tapes. X-rays with an energy of $12 \mathrm{keV}(\lambda=$ $1.035 \AA$ ) and a $15 \times 15 \mathrm{~cm}^{2}$ mosaic Mar CCD at a sample to detector distance of $2 \mathrm{~m}$ were 
used to measure the scattered intensity and a pin diode on the beam stop to measure the transmitted beam intensity. For each sample we collected five $1 \mathrm{~s}$ exposures and the collected $2 \mathrm{D}$ data were corrected and azimuthally averaged to obtain $\mathrm{I}(\mathrm{Q})$ data, where $\mathrm{Q}=4 \pi \sin \theta / \lambda$ with the scattering angle $2 \theta$ and the wavelength $\lambda$ of the X-rays.

The SAND instrument provides data in the $Q$ range of $0.004-0.5 \AA^{-1}$ in a single measurement by using a 40x40 $\mathrm{cm}^{2}$ position-sensitive ${ }^{3} \mathrm{He}$ detector and neutrons with wavelengths in the range of 1.5-14 $\AA$. The neat polymer gel samples of PentaPZ46-5, PentaPAA27-5, PentaPAA36-5 and the corresponding nanocomposite gel samples were prepared in $1 \mathrm{~m} \mathrm{M}$ Tris- $\mathrm{HCl}$ at $\mathrm{pH} 5.5$, in $\mathrm{D}_{2} \mathrm{O}$ solution. The gel samples were sealed in Suprasil cylindrical cells with $1 \mathrm{~mm}$ path length for the SANS measurements. The scattering data were corrected for empty cell scattering, detector sensitivity, and sample transmission. The differential scattering cross section $I(Q)$ was placed on an absolute scale in the units of $\mathrm{cm}^{-1}$

Details of the other experimental parameters and the sample preparation for the corresponding techniques can be found in Kanapthipillai et al., Chemistry of Materials, Supporting Information.

\subsection{Results and Discussion}

Polymer characterization. The molecular weight of the cationic PDEAEM 5 -Pluronic ${ }^{\circledR}$ F127PDEAEM $_{5}$ pentablock copolymer, synthesized by ATRP, was 14,570 by GPC with a narrow molecular weight distribution (PDI $<1.2)$. The cationic pentablock copolymer was subsequently betanized with 1,3-propane sultone in dry THF to give the PentaPZ zwitterionic polymer and the composition verified by solution NMR. The molecular weight of the PentaPAA polymer was 17,538 with a narrow molecular weight distribution $(\mathrm{PDI}<1.2)$. 
These PentaPZ and PentaPAA copolymers exhibit micellization, which is observed from the DSC thermograph (Figure 5.1) and thermo-reversible gelation due to the PPO block (Figures 5.2), and impart $\mathrm{pH}$ dependent solubility due to their ionic blocks.
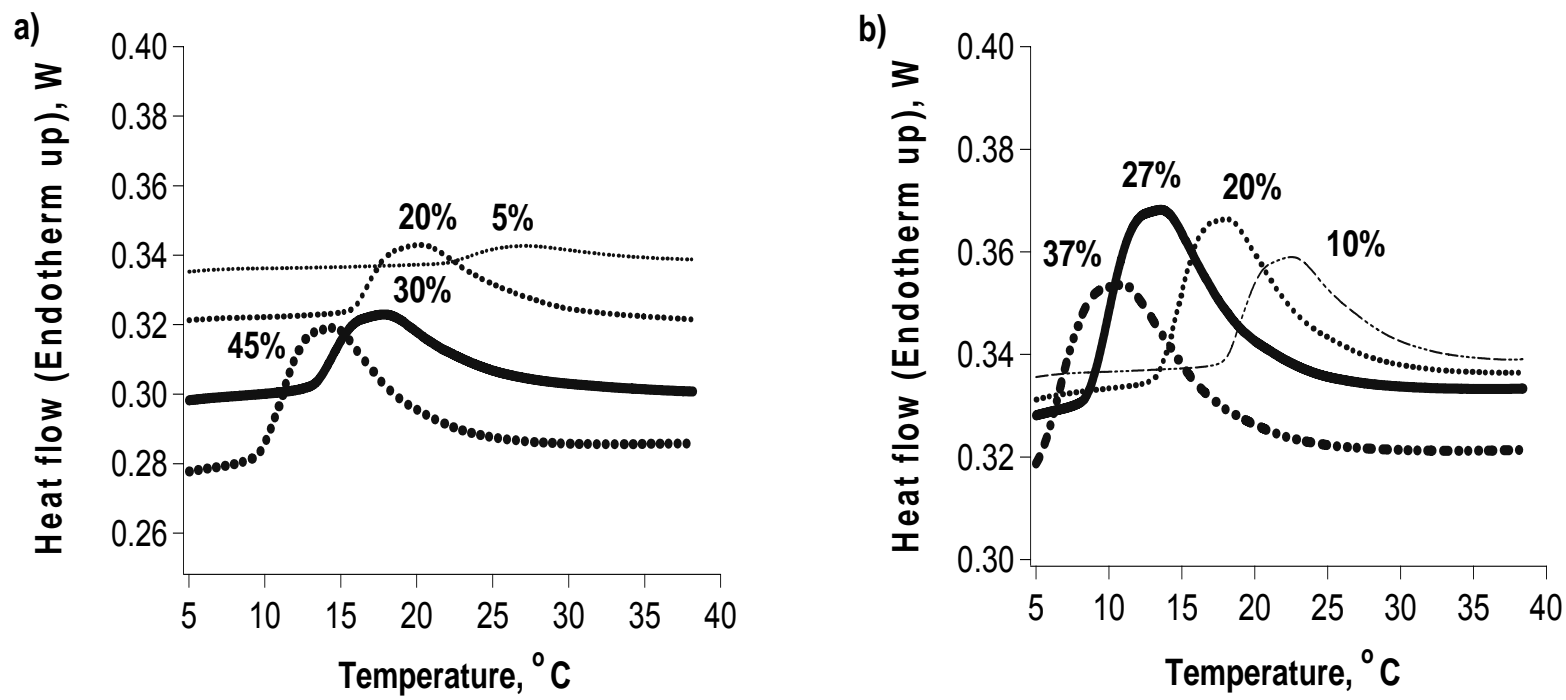

Figure 5.1. Endothermic micellization peaks of (a) PentaPZ (Mn, 15000) (b) PentaPAA (Mn 17538, PDI- 1.196) pentablock copolymers obtained from DSC thermographs.
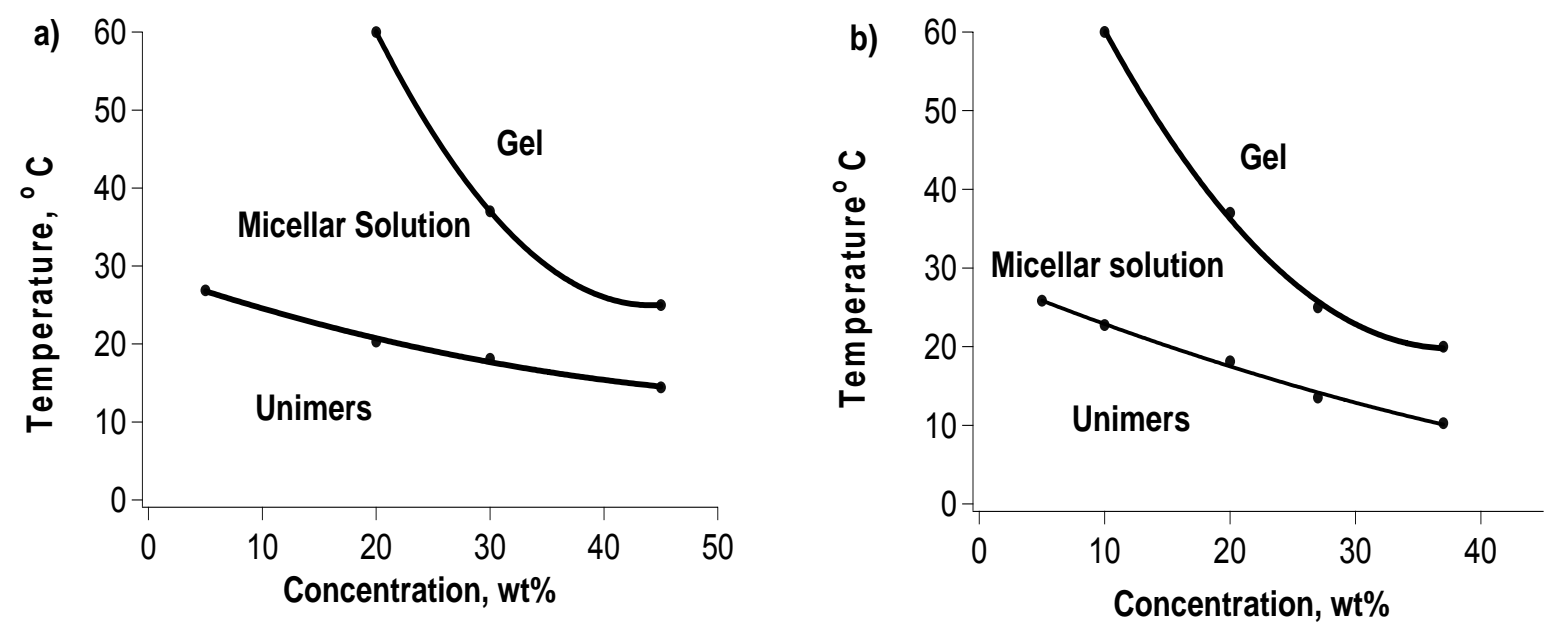

Figure 5.2. Phase diagrams of a) PentaPZ (Mn, 15000), b) PentaPAA (Mn 17538, PDI1.196) pentablock copolymers obtained from DSC thermographs and simple gel inversion techniques. 
The zwitterionic pentablock copolymer exhibits $\mathrm{pH}$ sensitivity over a wide $\mathrm{pH}$ range from $\mathrm{pH} \approx 2-11$ due to the sulfobetaine blocks, with an isoelectric point around $\mathrm{pH} 6.3$, where the concentrations of positive and negative charges are equal. The anionic pentablock copolymer on the other hand, is hydrophilic above its pKa (6.3) due to the acrylic acid side chains.

Formation of organic-inorganic nanocomposites. The thermogravimetric analysis of the $\mathrm{CaP} /$ hydrogel samples (Kanapathipillai et al.,Chemistry of Materials) showed approximately $30 \mathrm{wt} \%$ inorganic for the dried hydrogels, which is significantly larger than in our previous studies of calcium phosphate formation on cationic and polar templates, ${ }^{6}$ which yielded 6 or $15 \mathrm{wt} \%$ of inorganic component. The proof of nanocomposite formation was obtained by a combination of NMR, SAXS/SANS and TEM measurements. The NMR characterizations were performed using direct polarization studies of the polymer-nanocomposites for characterization of the inorganic phase ${ }^{21}$, two-dimensional ${ }^{1} \mathrm{H}^{-31} \mathrm{P}$ heteronuclear correlation NMR experiments with ${ }^{1} \mathrm{H}$ spin diffusion ${ }^{22,23,24}$ for the proof of nanocomposite formation and ${ }^{31} \mathrm{P}\left\{{ }^{1} \mathrm{H}\right\}$ HARDSHIP NMR ${ }^{25,26}$ measurements to quantify the thickness of the inorganic domain.

An example NMR spectra from the WISE experiments shown in Figure 5.3, shows that the polymer and inorganic phases are in close contact and the inorganic domains are in the nanometer size range (Courtesy Yanyan $\mathrm{Hu}$ ). In a nanocomposite, the polymer proton peaks appear within tens to hundreds of milliseconds of spin diffusion. The detailed NMR analysis can be found in Kanapathipillai et al.,Chemistry of Materials. 

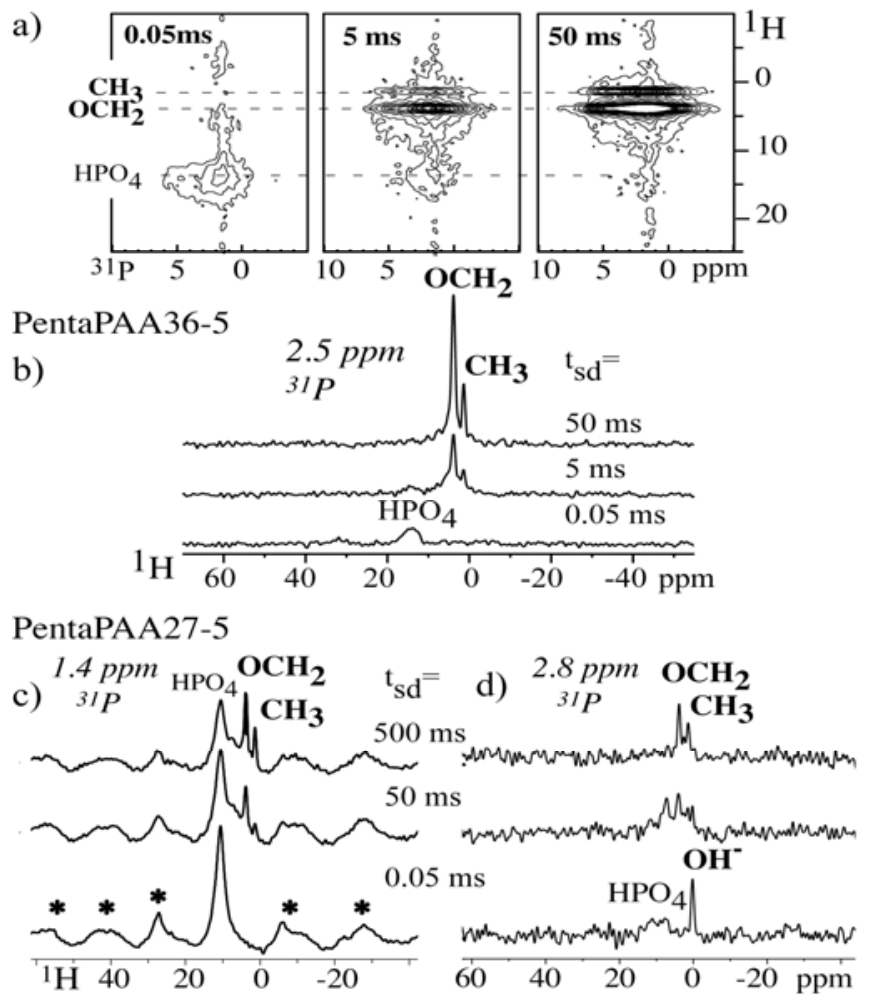

Figure 5.3. a) 2D "WISE" NMR spectra of PentaPAA36-5 with spin diffusion mixing time $\left(t_{\mathrm{sd}}\right)$ of $0.05 \mathrm{~ms}, 5 \mathrm{~ms}$, and $50 \mathrm{~ms}$. b) Cross-sections from the $2 \mathrm{D}$ spectra in a) at $2.5 \mathrm{ppm}{ }^{31} \mathrm{P}$. c, d): Cross-sections from 2D "WISE" spectra of PentaPAA27-5 with spin diffusion mixing times of $0.05 \mathrm{~ms}, 50 \mathrm{~ms}$ and $500 \mathrm{~ms}$, taken c) at $1.4 \mathrm{ppm}{ }^{31} \mathrm{P}$; spinning sidebands are marked by asterisks in the bottom trace; and d) at $2.8 \mathrm{ppm}^{31} \mathrm{P}$. (Adapted from Kanapathipillai et al., Chemistry of Materials)

SAXS and SANS: Evidence of templating. SAXS and SANS provide direct information on the morphology and ordering of structures in the gels and nanocomposites. In SANS, the contrast is due to the nuclear scattering length difference between the $\mathrm{D}_{2} \mathrm{O}$ and the polymer/inorganic phase. As shown in Figure 5.4a, the self-assembled zwitterionic PentaPZ46-5 copolymer gels both with and without calcium phosphate display distinct first order diffraction peaks in SANS data. The second order peak position in relation to the first order peak $\left(\mathrm{Q}^{*}\right)$ in the neat polymer gel clearly indicates hexagonal close packing of cylinders in the ordering of micelles. However, in the case of the nanocomposite gel, the first order diffraction peak broadens, making the second order peak less visible, presumably due to disorder introduced by the templating of calcium phosphate on the polymer phase. 
The SAXS data in Figure 5.4b show similar diffraction peaks both without and with calcium phosphate. The peak intensity is greatly increased in the composite, which proves that a significant fraction of the high-electron density inorganic phase is templated by the block copolymer micelles and thus enhances their structure factor. The diffraction peaks are observed at $\mathrm{Q} / \mathrm{Q}^{*}=1: \sqrt{3}: \sqrt{7}: \sqrt{12}$, indicating hexagonal close packing of rod-like polymer micelles consistent with the SANS data. Using the $\mathrm{Q}^{*}$ value for the gels with cylinder phase the lattice spacing $d=(4 / 3)^{1 / 2}\left(2 \pi / Q^{*}\right)$ can be determined. Furthermore, in the low $Q$ region of the SAXS data the scattering intensity $\mathrm{I}(\mathrm{Q})$ varies as $\mathrm{Q}^{-1.5}$ suggesting that both the neat and the nanocomposite gels have rod-like structures, in agreement with the TEM data below.

The lattice spacings (the characteristic distance between the centers of the cylinders) from the SANS data of the neat and the calcium phosphate templated PentaPZ46-5 polymer gels were around $18.5 \mathrm{~nm}$, indicating little effect of the inorganic component on the superstructure. This may be due to the relatively small inorganic volume fraction in the nanocomposite. Similar trends were observed in the SANS data for PentaPAA27-5 (Figures $5.4 \mathrm{c}, \mathrm{d}$ ) and PentaPAA36-5 (Figure 5.4 e,f) polymer-gel nanocomposites. However, in contrast to the PentaPZ46-5 gels, the PentaPAA gels exhibit sharper peaks in the SANS patterns, indicating a higher level of ordering. Further the PentaPAA36-5 neat polymer show diffraction peaks at $\mathrm{Q} \mathrm{Q}^{*}=\sqrt{3}: 2: \sqrt{8}$, indicating $\mathrm{FCC}$ packing of spherical polymer micelles consistent with the SAXS and TEM data. Using the $\mathrm{Q}^{*}$ value for the gels with FCC phase the lattice spacing $d=2 \pi / Q^{*}$ can be determined. The $d$-spacings in the gel structures of PentaPAA27-5 and PentaPAA36-5 are around $16 \mathrm{~nm}$, but there was a slight reduction in the d-spacing in the presence of calcium phosphate when compared to the neat polymers. In the case of PentaPAA27-5 the polymer phase has ionic character and the broadening of the peak in the SANS pattern for the neat polymer gel might be due to electrostatic repulsion between the micelles leading to a looser packing of particles. The sharper peaks in the presence of 
calcium phosphate might be due to charge neutralization in the PentaPAA gels leading to more ordered dense packing.
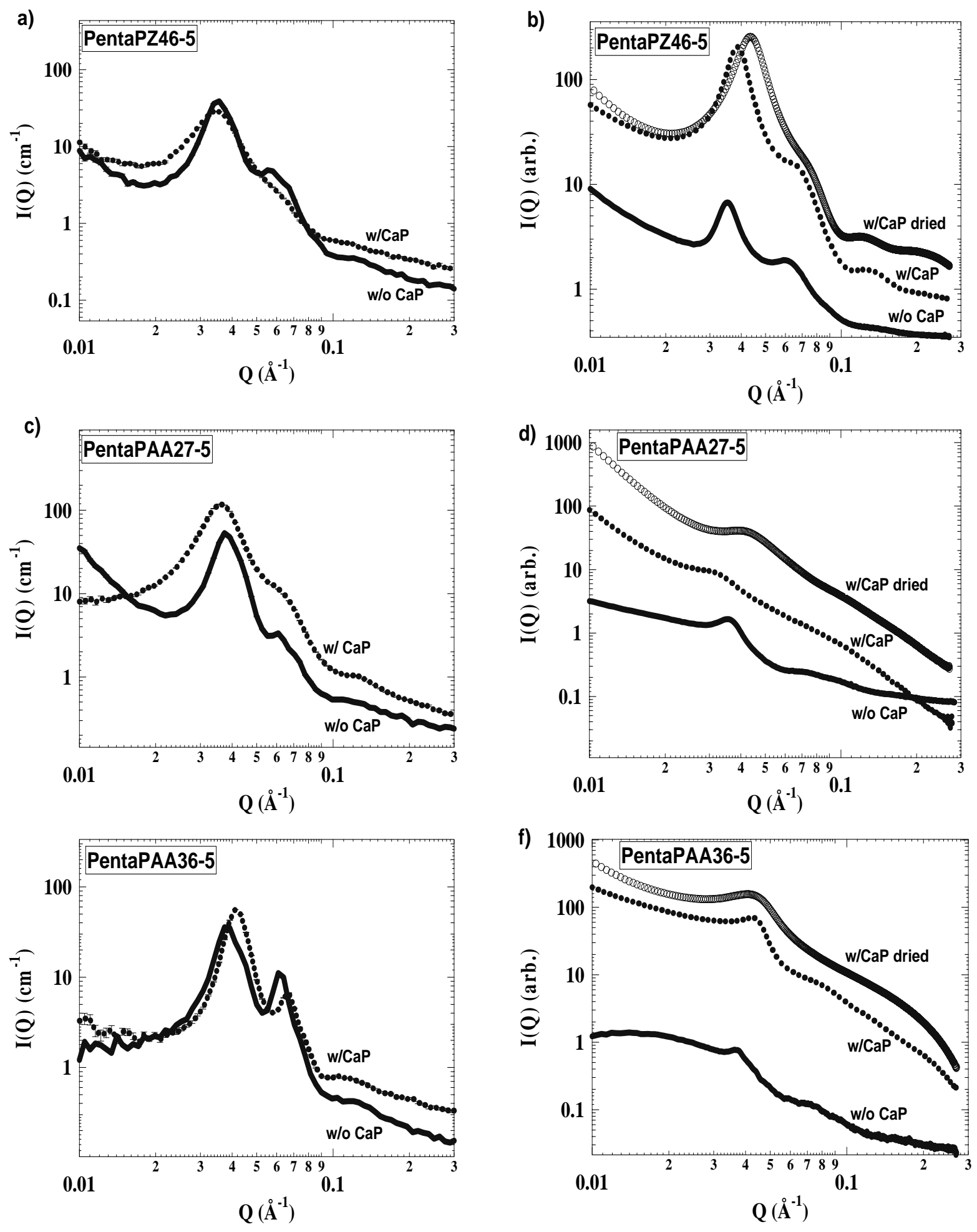
Figure 5.4. SANS and SAXS data of the three nanocomposites. a): SANS and b) SAXS of PentaPZ46-5 polymer inorganic nanocomposites gels at $\mathrm{pH} 5.0$

c) SANS and d)) SAXS of PentaPAA27-5 polymer nanocomposite gels.

e) SANS and f) SAXS of PentaPAA36-5 polymer inorganic nanocomposites gels.

The SAXS data show relatively low intensity for the neat PentaPAA polymer gels due to low X-ray contrast between polymer and water. In the presence of the inorganic component, higher scattering intensity was again observed for PentaPAA36-5 with a scattering peak corresponding to a d-spacing of $\sim 15 \mathrm{~nm}$, proving templating of calcium phosphate on the polymer micelles. Furthermore, the SAXS curves of both PentaPAA nanocomposites show power-law scattering with a higher exponent $(\sim 4)$ in the low $\mathrm{Q}$ region. This suggests the presence of larger inorganic aggregates with 3-dimensional morphology. The higher power-law exponent in the case of PentaPAA27-5 when compared to PentaPAA36-5 suggests the presence of larger calcium phosphate aggregates in the former, in agreement with the NMR data. It is interesting to note that the small-angle upturn in the SAXS curves could be attributed not only to larger inorganic particles, but alternatively to a superstructure of the mineralized micelles, such as $0.2-\mu \mathrm{m}$ diameter aggregates. The TEM data show that larger inorganic particles are indeed present. The dried nanocomposite gel sample exhibits SAXS patterns similar to those of the gel samples. However, a small shift in the scattering patterns towards larger Q-values (smaller spacings) was observed, which is expected due to the removal of water.

Nanocomposite morphology by TEM. Figures 5.5a and 5.6a show the transmission electron micrographs of $5 \mathrm{wt} \%$ PentaPAA and PentaPZ polymers (TEM, and XRD studies conducted by collaborator Yusuf Yusufoglu). The samples were prepared by diluting the neat gels in deionized water. The images revealed approximately $15-\mathrm{nm}$ diameter spheroidal micelles, in

agreement with the SAXS and SANS results and our previous studies ${ }^{6}$. TEM image of PentaPAA36-5 nanocomposite gel diluted to $5 \mathrm{wt} \%$ polymer are shown in Figure 5.5b. 
STEM-EDX measurements indicate that both calcium and phosphate (dark regions in Figure 5.5b) are present around the micelles. The atomic ratio of calcium to phosphorus for PentaPAA27-5 was found to be about 1 , quite close to that of brushite $(\mathrm{Ca} / \mathrm{P}=1)$, while for PentaPAA36-5, in addition to regions with $\mathrm{Ca} / \mathrm{P}=1$, some areas with a $\mathrm{Ca} / \mathrm{P}$ ratio of 1.9 were also observed, indicating there is another calcium phosphate phase other than brushite, consistent with NMR. Further, Figure 5.5c shows a TEM micrograph of plate-like calcium phosphate crystallites collected from the bottom of the redissolved polymer in PentaPAA365, which was also observed from spin-diffusion NMR (Kanapathipillai et al., Chemistry of Materials). Figure 5.5d, shows a high-angle annular dark field (HAADF) image of PentaPAA27-5, where the calcium phosphate coating of some of the micelles can be seen more clearly.
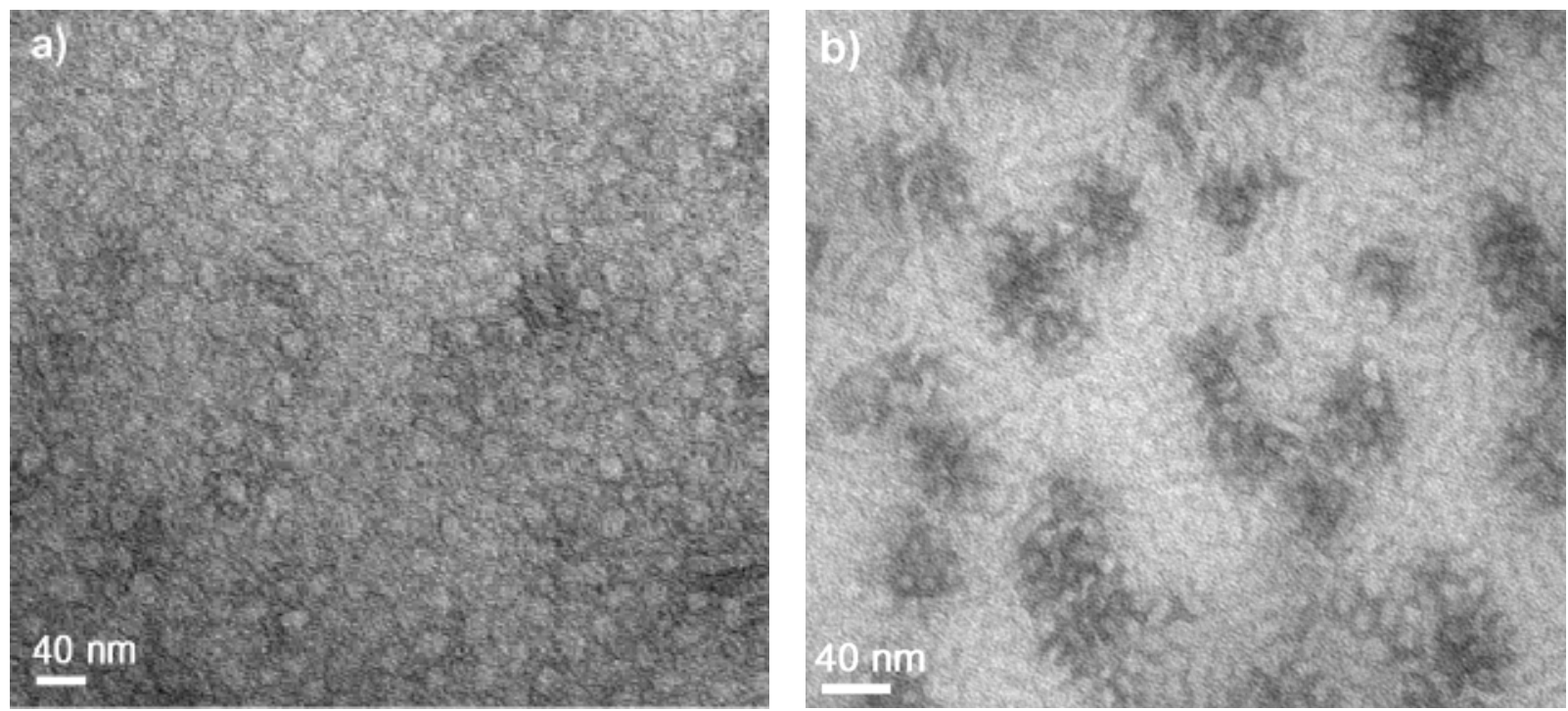

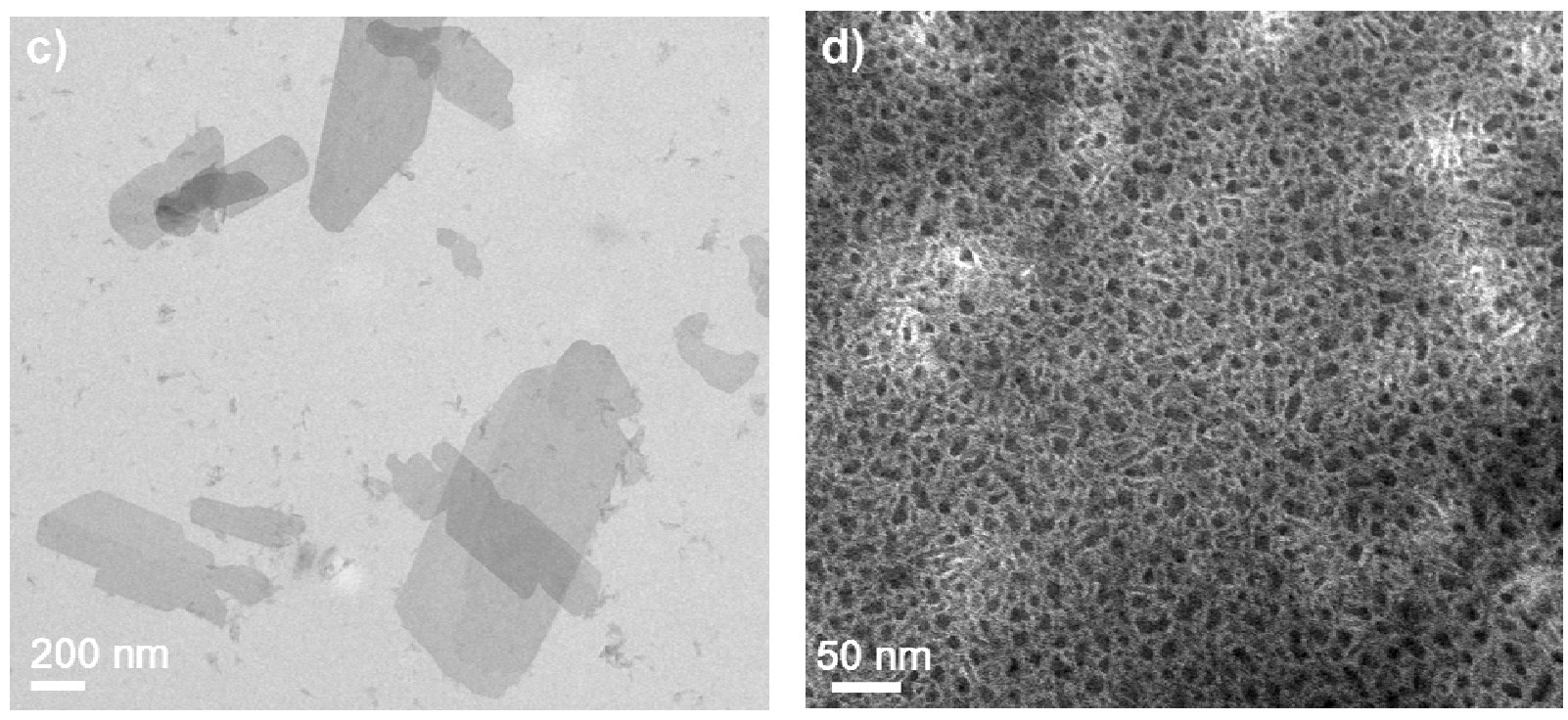

Figure 5.5. Transmission electron micrographs of stained a) $5 \mathrm{wt} \%$ PentaPAA polymer prepared in deionized water, b) PentaPAA36-5 nanocomposite deposited from a suspension diluted to $5 \mathrm{wt} \%$ polymer, c) crystallites collected from the bottom of the vial containing the diluted PentaPAA36-5 material, and d) high-angle annular dark field (HAADF) image of PentaPAA27-5 sample. Dark spots: polymer.(Adapted from Kanapathipillai et al., Chemistry of Materials)
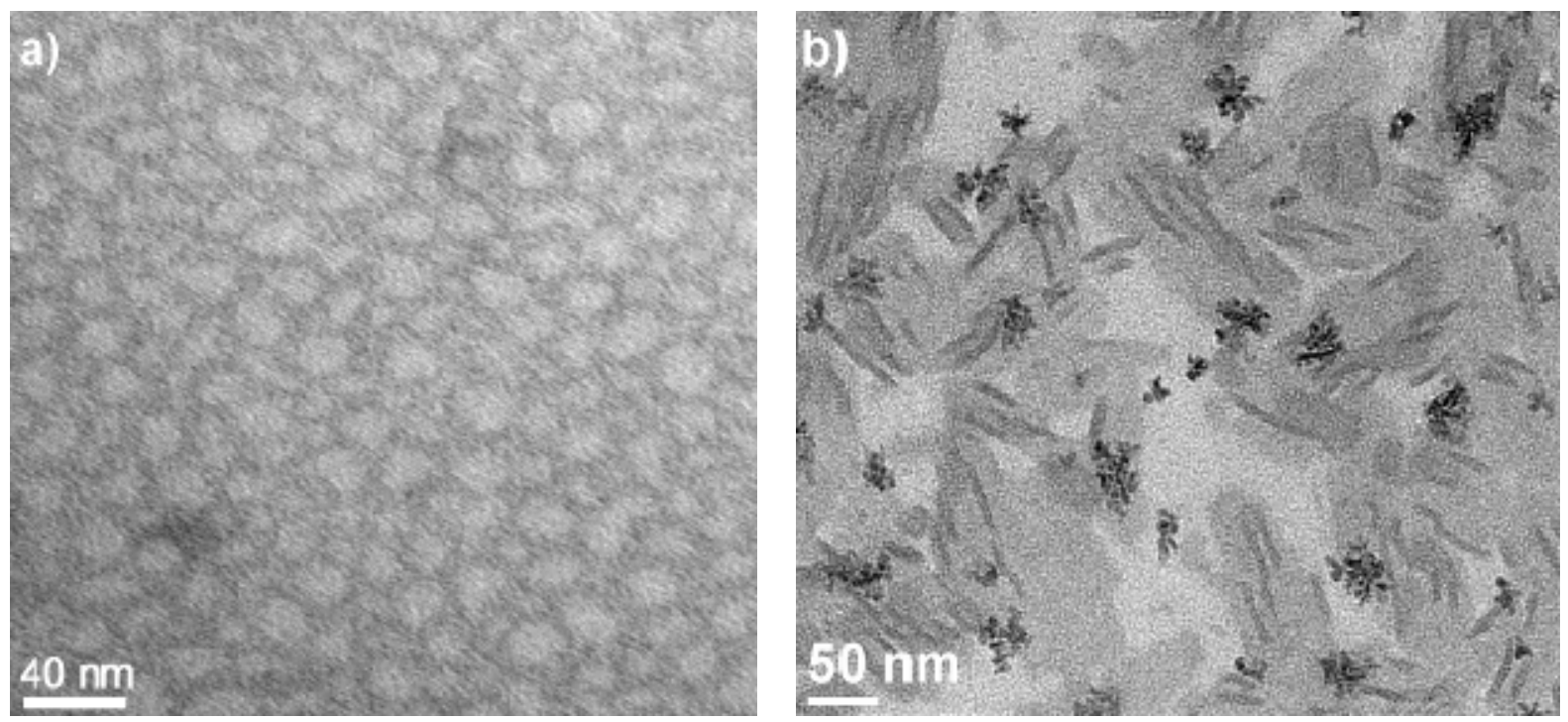

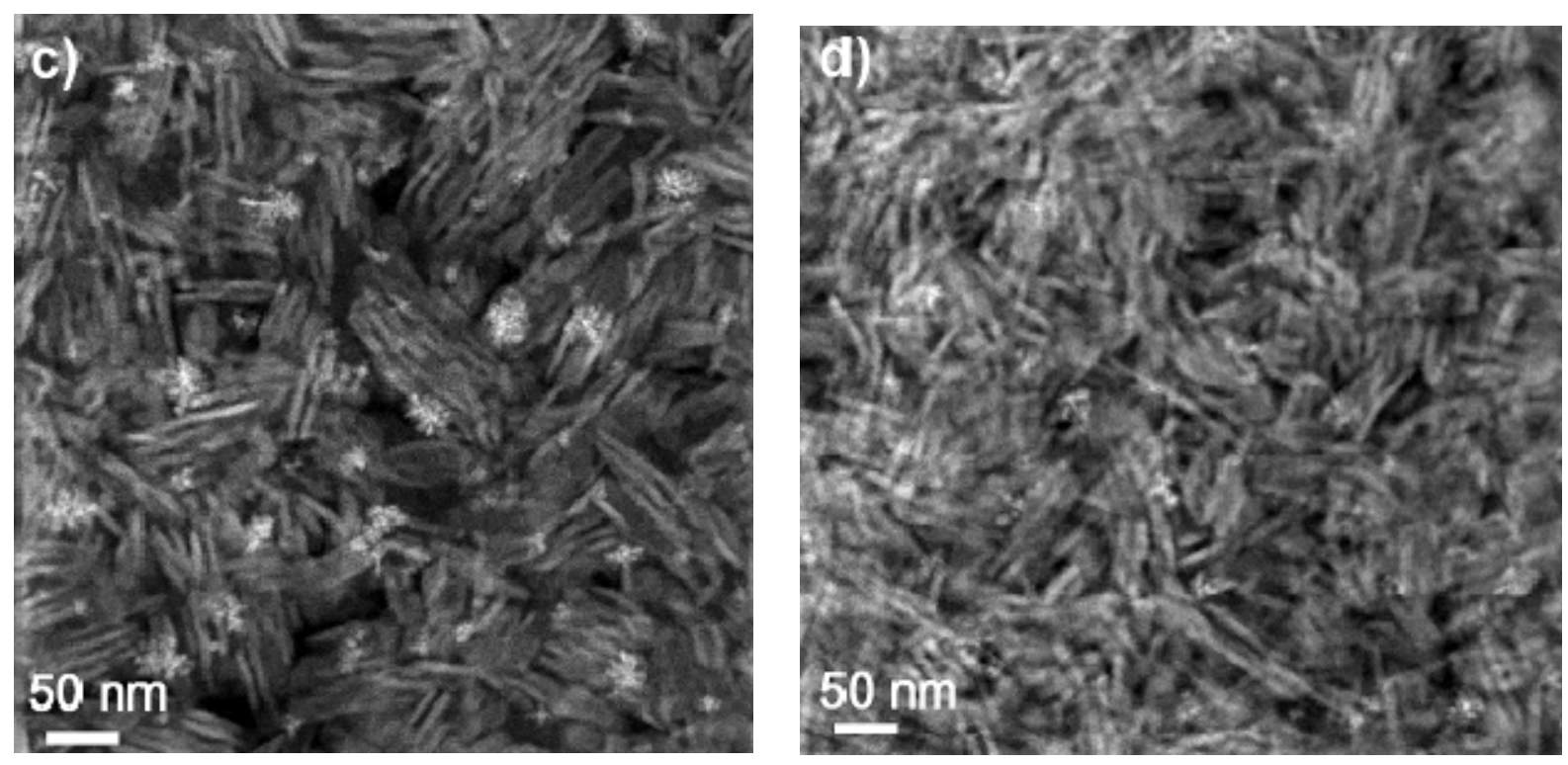

Figure 5.6. Transmission electron micrographs of stained a) $5 \mathrm{wt} \%$ PentaPZ polymer prepared in deionized water, b) PentaPZ46-5 nanocomposite deposited from a suspension diluted to $5 \mathrm{wt} \%$ polymer, and (c, d) HAADF images of the same sample. (Adapted from Kanapathipillai et al., Chemistry of Materials)

TEM micrographs of PentaPZ46-5 are shown in Figure 5.6. The neat polymer ( $5 \mathrm{wt} \%$ in deionized water) revealed the typical $\sim 15-\mathrm{nm}$ diameter spherical micelles (Figure 5.6a) while the PentaPZ46-5 nanocomposite, exhibited rod-like calcium phosphate nanoparticles (Figures 5.6 b-d).

\section{Identification of the calcium phosphate phase}

X-ray diffraction pattern of PentaPAA27-5 revealed the typical reflections for crystalline synthetic brushite $\left(\mathrm{CaHPO}_{4} \cdot 2 \mathrm{H}_{2} \mathrm{O}\right)$ (ICDD card \#09-0077). XRD results for PentaPAA36-5 showed only broad reflections indicating that the calcium phosphate phase might be amorphous, nanocrystalline, or a minor volume fraction compared to the polymer. Moreover, the characteristic peaks of natural brushite (ICDD card \#11-0293), a mineral normally only found in caves ${ }^{27}$ were observed for PentaPZ46-5, and this is similar to our 
previous results with PDEAEM modified Pluronic ${ }^{\circledR}$ F127 pentablock gel composite as templates. $^{6}$

After vacuum drying of the hydrogel samples, the natural brushite peaks disappeared for PentaPZ46-5, while weak HAp peaks appeared in addition to synthetic brushite peaks for PentaPAA27-5 in agreement with NMR. Sharp peaks of brushite and sodium calcium hydrogen phosphate were observed for PentaPAA36-5 after drying. Using the Scherrer equation, brushite crystal sizes of $>40 \mathrm{~nm}$ and $>45 \mathrm{~nm}$ were estimated from the widths of the Bragg peaks for the vacuum dried PentaPAA36-5 and PentaPAA27-5, respectively, which is also confirmed from NMR analysis. The different inorganic phases formed by the different pentablock copolymer templates suggest the ionic nature of the polymer influences the calcium phosphate crystal formation. The detailed XRD, NMR and STEM-EDX phase analysis of the nanocomposite samples is described in (Kanapathipillai et al., Chemistry of Materials).

Dynamic-mechanical measurements. Dynamic strain controlled rheological measurements were performed on polymer nanocomposite gels to test the stiffness of the formed materials (Figure 5.7). Measurements indicated an increase in modulus with the inorganic nanocrystals compared to polymer gels alone. The polymer gels alone were not stiff enough due to charge repulsions by the ionic side chains, where the moduli ranged from 0.01-10 $\mathrm{Pa}$. With the formation of the inorganic nanocrystals, the nanocomposites show an increase of modulus by several orders of magnitude depending on the shear rate. Such a large increase may be attributed to neutralization of surface charges on the micelle surface by calcium phosphate phase leading to aggregation of the nanoparticles, or coating of soft polymeric micelles by rigid inorganic particles, or both. 

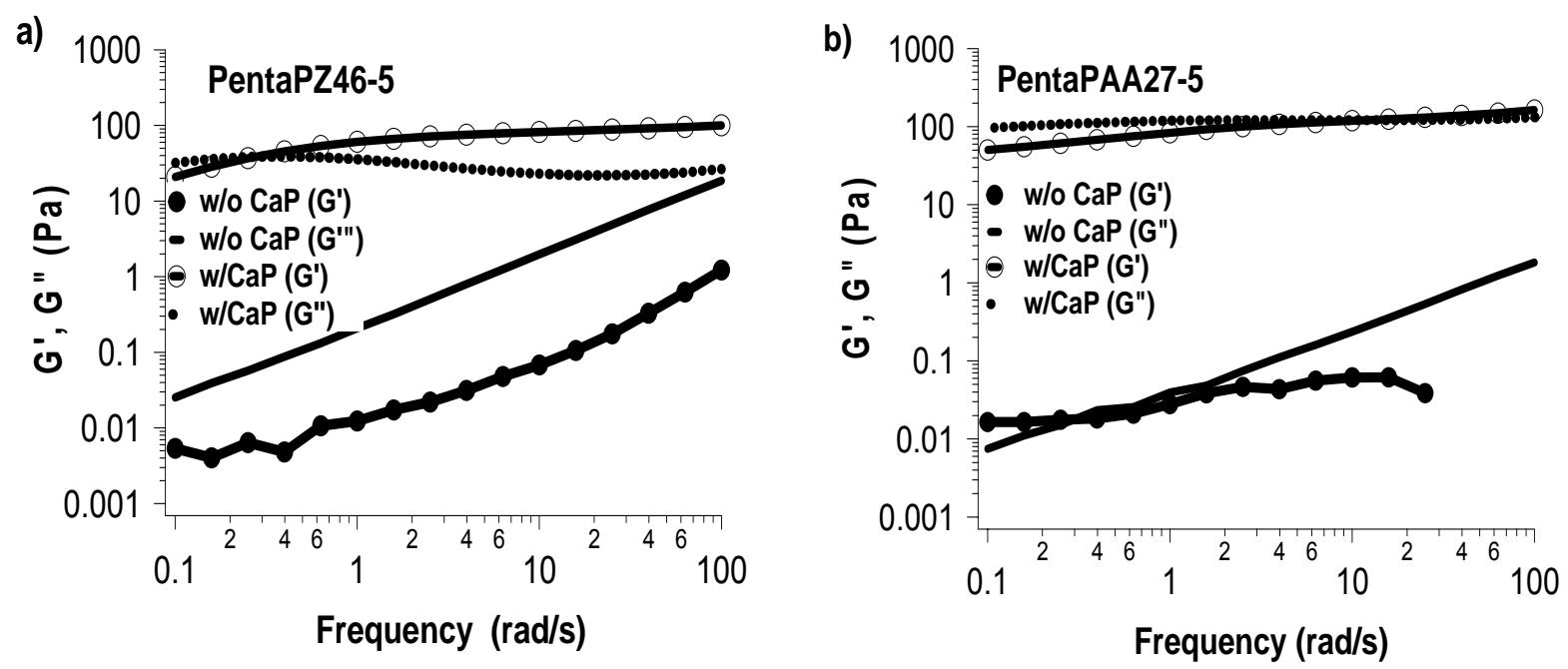

Figure 5.7. a) Dynamic strain controlled frequency sweep of PentaPZ46-5 and b) PentaPAA27-5 pentablock copolymers with and without calcium phosphate nanocrystals.

\subsection{Conclusion}

Scattering measurements and NMR revealed the formation of nanosized ordered inorganic nanocrystals. Rheological measurements indicated an increase in modulus due to the presence of inorganic crystals in the polymer template. The inorganic fraction in the template was found to be around $30 \%$ for all the templates. This is a significant increase compared to our earlier polymer cationic and polar templates, which only accommodated around $15 \%$ inorganic under extremely low $\mathrm{pH}$ conditions. These results suggest that for inorganic precipitation, ionic interactions are important. Further carboxylic groups and zwitterionic groups are better for the inorganic templated precipitation compared to cationic and polar groups. The self-assembling ionic block copolymer templates exhibited ideal properties for the bottom-up approach to nanocomposite design. We have reported the influence of key design and experimental parameters on the templating of nanoparticles by 
ionic polymeric gel superstructures. The initial ionic polymer templates will be a good guideline for our final goal of mimicking hierarchical natural structures.

\subsection{Acknowledgements}

This work was supported by the U.S. Department of Energy under contract number DE-

AC02-07CH11358. This work benefited from the use of SAND at IPNS, funded by the U.S.

DOE, Office of Science, Office of Basic Energy Science under contract DE-AC02-

06CH11357. We wish to thank Matthew J. Kramer for helpful discussion of the TEM results.

\subsection{References}

1. Song, J.; Saiz, E.; Bertozzi, C. R. J. Eur. Ceram. Soc. 2003, 23, 2905-2919.

2. $\quad$ Song, J.; Malathong, V.; Bertozzi, C. R. J. Am. Chem. Soc. 2005, 127, 3366-3372.

3. Rusu, V. M.; Ng, C.-H.; Wilke, M.; Tiersch, B.; Fratzl, P.; Peter, M. G. Biomaterials 2005, 26, 5414-5426.

4. $\quad$ Pozzo, D. C.; Hollabaugh, K. R.; Walker, L. M. J. Rheol.(N.Y.) 2005, 49, 759-782.

5. Pozzo, D. C.; Walker, L. M. Colloids Surf., A 2007, 294, 117-129.

6. Enlow, D.; Rawal, A.; Kanapathipillai, M.; Schmidt-Rohr, K.; Mallapragada, S.; Lo, C. T.; Thiyagarajan, P.; Akinc, M. J. Mater. Chem. 2007, 17, 1570-1578.

7. Du, C.; Cui, F. Z.; Zhang, W.; Feng, Q. L.; Zhu, X. D.; De Groot, K. J. Biomed. Mater. Res. 2000, 50, 518-527.

8. $\quad$ Hartgerink, J. D.; Beniash, E.; Stupp, S. I. Science 2001, 294, 1684-1688.

9. Mann, S. Principles and Concepts in Bioinorganic Materials Chemistry 2001, 240 pp.

10. Tanahashi, M.; Matsuda, T. J. Biomed. Mater. Res. 1997, 34, 305-315.

11. Miyazaki, T.; Ohtsuki, C.; Akioka, Y.; Tanihara, M.; Nakao, J.; Sakaguchi, Y.; Konagaya, S. J. Mater. Sci.: Mater. Med. 2003, 14, 569-574.

12. Spanos, N.; Deimede, V.; Koutsoukos, P. G. Biomaterials 2001, 23, 947-953.

13. Anderson, J. A.; Travesset, A. Macromolecules 2006, 39, 5143-5151.

14. Knorowski, C. D.; Anderson, J. A.; Travesset, A. Phys. Rev. Lett. 2008, submitted.

15. Barbani, N.; Lazzeri, L.; Cristallini, C.; Cascone, M. G.; Polacco, G.; Pizzirani, G. J. Appl. Polym. Sci. 1999, 72, 971-976.

16. Iwatsubo, T.; Kusumocahyo, S. P.; Kanamori, T.; Shinbo, T. J. Appl. Polym. Sci. 2006, 100, 1465-1470.

17. Iwatsubo, T.; Sumaru, K.; Kanamori, T.; Shinbo, T.; Yamaguchi, T. Biomacromolecules 2006, 7, 95-100.

18. Determan, M. D.; Cox, J. P.; Seifert, S.; Thiyagarajan, P.; Mallapragada, S. K. Polymer 2005, 46, 6933-6946.

19. Wanka, G.; Hoffmann, H.; Ulbricht, W. Macromolecules 1994, 27, 4145-4149.

20. Petrov, A. I.; Antipov, A. A.; Sukhorukov, G. B. Macromolecules 2003, 36, 1007910086 
21. Rothwell, W. P.; Waugh, J. S.; Yesinowski, J. P. J. Am. Chem. Soc. 1980, 102, 26372643.

22. Hou, S. S.; Beyer, F. L.; Schmidt-Rohr, K. Solid State Nucl. Magn. Reson. 2002, 22, 110-127.

23. Hou, S. S.; Bonagamba, T. J.; Beyer, F. L.; Madison, P. H.; Schmidt-Rohr, K. Macromolecules 2003, 36, 2769-2776.

24. Schmidt-Rohr, K. Macromolecules 1992, 25, 3273-3277.

25. Gullion, T.; Schaefer, J. JMR 1989, 81, 196-200.

26. Schmidt-Rohr, K.; Rawal, A.; Fang, X.-W. J. Phys. Chem. 2007, 126, 054701.

27. Murray, J. W.; Dietrich, R. V. Am. Mineral. 1956, 41, 616-626. 


\section{CHAPTER 6. BIOINSPIRED SYNTHESIS OF SELF-ASSEMBLED}

\section{CALCIUM PHOSPHATE NANOCOMPOSITES USING BLOCK COPOLYMER-PEPTIDE CONJUGATES}

(This is a collaborative work submitted to Journal of Materials Research and has been modified to focus on my contribution to the work)

Yusuf Yusufoglu ${ }^{\mathrm{a},+}$, Yanyan $\mathrm{Hu}^{\mathrm{a},+}$, Mathumai Kanapathipillai ${ }^{\mathrm{a}}$, YaQiao Wu ${ }^{\mathrm{a}}$, Matthew Kramer $^{\mathrm{a}}$, Yunus Kalay ${ }^{\mathrm{a}}$, P. Thiyagarajan ${ }^{\mathrm{b}}$, Mufit Akinc ${ }^{\mathrm{a}}$, Klaus Schmidt-Rohr ${ }^{\mathrm{a}}$ and Surya Mallapragada ${ }^{\mathrm{a}, *}$

\subsection{Abstract}

Thermoreversibly gelling block copolymers conjugated to hydroxyapatite nucleating peptides (DSKSDSSKSESDSS) were used to template the growth of inorganic calcium phosphate in aqueous solutions. The effect of growth and nucleation of calcium phosphate mineral by these peptide conjugated polymers was studied using NMR, FTIR, transmission electron microscopy, X-ray diffraction and small-angle scattering techniques. Three different polymer templates were used with varying charges on the polymer chains (non-ionic, anionic and zwitterionic), to investigate the role of charge on mineralization. All the polymerinorganic solutions exhibited thermoreversible gelation above room temperature. Nanocomposite formation was confirmed by solid-state NMR, and other complementary methods identified the inorganic component as hydroxyapatite. The morphology of the hydroxyapatite was identified as thin, elongated crystals from small angle X-ray scattering and electron microscopy methods. The inorganic content was found to be of $30-45 \mathrm{wt} \%$ (based on the mass of the dried gel at $\sim 200^{\circ} \mathrm{C}$ ) in the various samples from 
thermogravimetric measurements. Our work offers routes for bioinspired bottom-up approaches for the development of novel self-assembling injectable nanocomposite biomaterials for potential orthopedic applications.

\footnotetext{
${ }^{\mathrm{a} A m e s ~ L a b o r a t o r y, ~} 144$ Spedding Hall, Ames, IA 50011

${ }^{b}$ Advanced Photon Source, Argonne National Laboratory, 9700 S. Cass Ave, Argonne, IL 60439

${ }^{+}$Both authors contributed equally to the work
}

\subsection{Introduction}

Recently many investigations been carried out to explore ways to mimic biomineralization $^{1-4}$ and to understand the mechanism(s) involved in it with biological macromolecules (e.g., proteins, enzymatic biocatalysts, and other biopolymers) ${ }^{5-9}$ Numerous studies have appeared on the in vitro synthesis and characterization of biominerals including calcium phosphate, calcium carbonate and silica. ${ }^{10-15}$ All these studies have focused on templating at the nanoscale or at the macroscale, but mostly to the surface of the template.

Controlling the structure at the molecular level as well as impressing hierarchical order over multiple length scales as seen commonly in Nature is a formidable challenge that has not been achieved yet, but significant progress is being made in these directions. Polymer templating has the advantage of molecular level control and a promise to extend to the macro scale by hierarchical ordering. Our recent work has shown that synthetic block copolymers that undergo self assembly at multiple length scales can serve as effective templates for precipitation of calcium phosphates on nanoscale micelles, which can self-assemble further into macroscale gels and solids. ${ }^{16}$ This is one of the first approaches for bottom-up design of macroscale composites with hierarchical order down to the nanoscale completely by selfassembly. However, the use of polymer templates alone does not provide biological 
specificity for controlled growth of the inorganic phase.

In Nature it is believed that, that the hydroxyapatite formation is greatly influenced by the matrix proteins, and it is believed that aminoacids such as serine, glutamic acid and aspartic acid play a major role in calcium phosphate minieralization. ${ }^{17}$ Further, it was reported that a 14 aminoacid peptide with a sequence of (DSKSDSSKSESDSS) enhances nucleation and growth of hydroxyapatite. ${ }^{17}$

Hence, here we report the synthesis of thermoreversibly self-assembling polymer templates with attached hydroxyapatite-nucleating peptides ${ }^{17}$ which were used to control the biomineralization process and to form polymer-hydroxyapatite nanocomposites completely by self-assembly. These novel systems that transition from liquid to solid at physiological temperatures could form the basis for injectable nanocomposites for orthopedic repair, such as in bone defects, where it could conform to the shape of the defect easily. A variety of complementary techniques including solid-state NMR, small angle scattering and transmission electron microscopy are used in this study to elucidate the structure and composition of these bioinspired nanocomposites.

\subsection{Materials and Methods}

The hydroxyapatite nucleating peptide (DSKSDSSKSESDSS), with a molecular weight of $1445 \mathrm{Da}$ and theoretical pI of 4.23, was synthesized by Genscript Corporation, NJ, with a purity of $\sim 95 \%$ and used as obtained. All other materials used were obtained from either Sigma Aldrich or Fisher scientific and of high purity.

\section{Synthesis and Characterization of Block Copolymers and Their Conjugates}

Polymer synthesis method and scheme. Pluronic F127 (poly(ethyelene oxide)-bpoly(propylene oxide)-b-poly(ethylene oxide)) with a molecular weight of 12,600 was used 
to synthesize Pluronic-based anionic (PentaPAA) $\left(\mathrm{M}_{\mathrm{n}}=17,458\right.$, PDI 1.14) and zwitterionic (PentaPZ) $\left(\mathrm{M}_{\mathrm{n}}=15,100\right.$, PDI 1.17) pentablock copolymers using an atom transfer radical polymerization (ATRP) technique as previously reported. ${ }^{18,19}$ These polymers are shown in Scheme 1. The Pluronic and PentaPZ end groups were modified to obtain carboxyl end groups as described below. The Pluronic and the two pentablock copolymers were then conjugated with the hydroxyapatite binding peptide using N-hydroxyl succinimide (NHS) chemistry. ${ }^{20,21}$ The peptide copolymer conjugation schemes used are shown below.

Scheme 1. Block copolymer structures

\section{Pluronic F127}

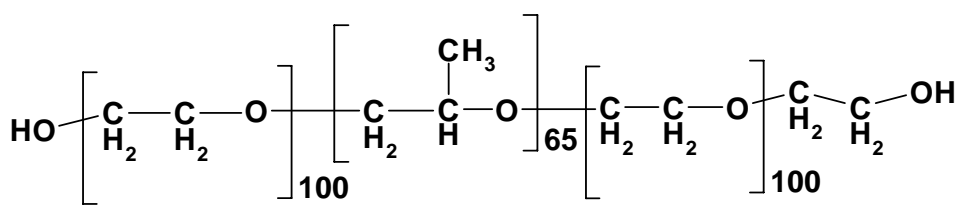

\section{PentaPZ}

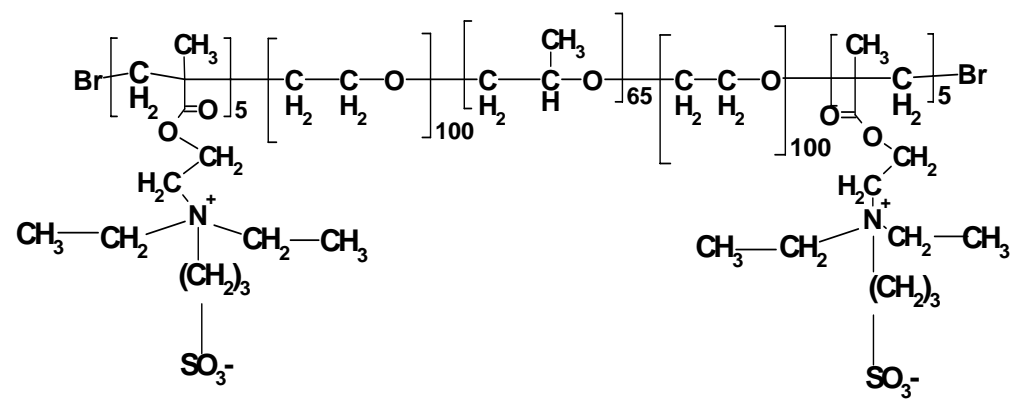

\section{PentaPAA}

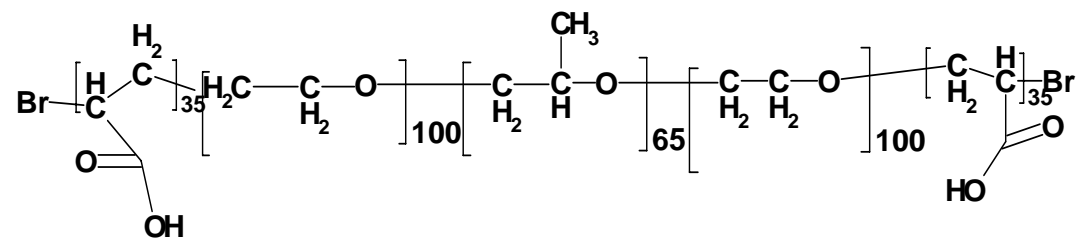


Scheme 2. Pluronic end group modification

\begin{tabular}{ll}
$-\mathrm{R}-\mathrm{OH}$ & Esterification \\
\cline { 2 - 2 } & $\begin{array}{l}\text { Succinic anhydride }, \\
\text { pyridine at } 40{ }^{\circ} \mathrm{C} \text { for } 24 \text { hours }\end{array}$
\end{tabular}$-\mathrm{R}-\mathrm{COOH}$

Scheme 3. PentaPZ end group modification

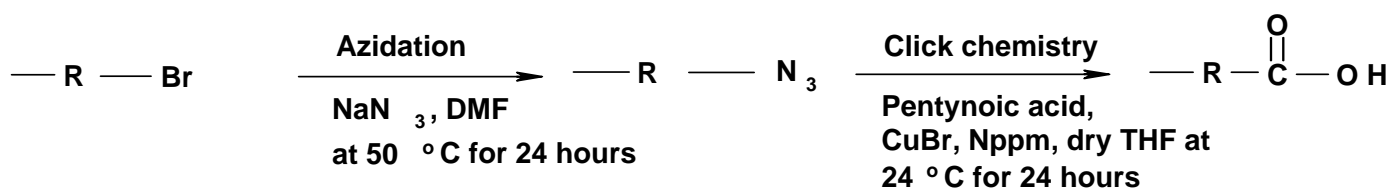

R- PentaPZ copolymer

Scheme 4. Pluronic and Pentablock copolymers peptide conjugation

a. NHS chemistry on carboxyl endgroups of Pluronic and PentaPZ

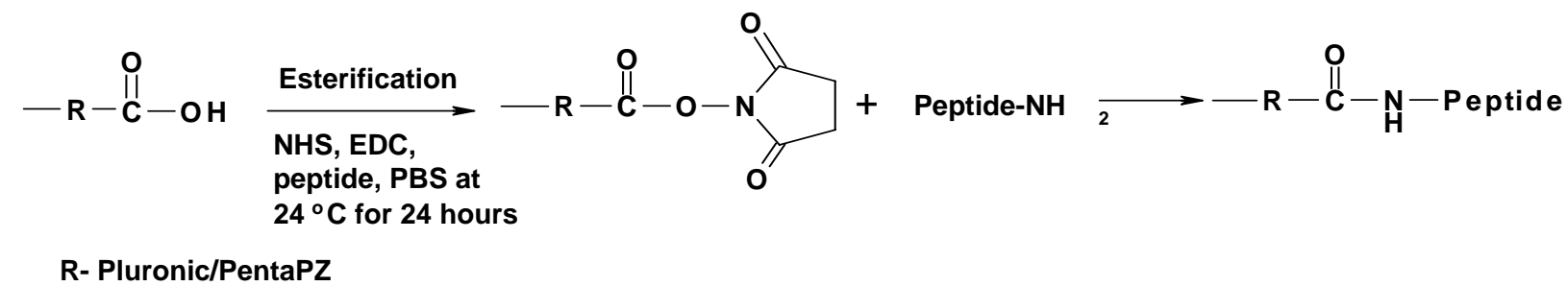

b. NHS chemistry on acrylic acid groups of PentaPAA

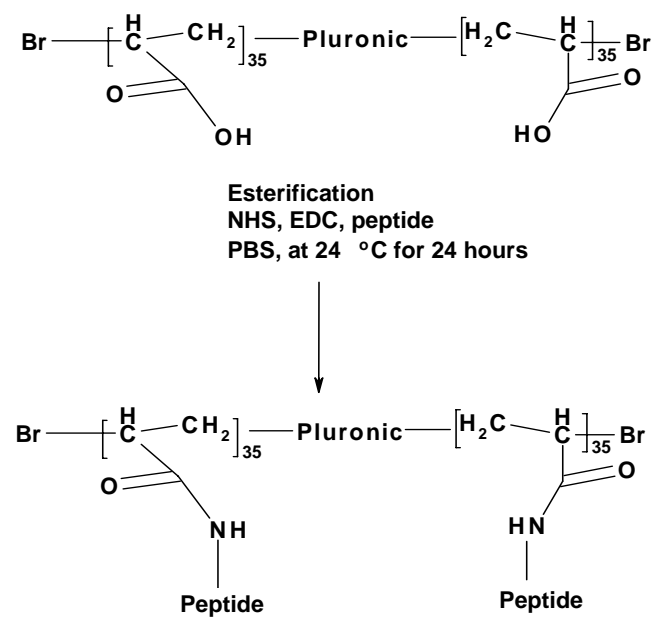

Note: Here peptides were attached randomly to acrylic acid groups.

Only few acrylic acids blocks were conjugated with peptides. 
Pluronic end group modification (Scheme 2). The hydroxyl end groups of Pluronic were converted to carboxyl groups by treating with succinic anhydride in pyridine as reported in the literature. ${ }^{22}$ In short, Pluronic $(32 \mathrm{~g}, 2.5 \mathrm{mmol})$ and succinic anhydride $(1 \mathrm{~g}, 10 \mathrm{mmol})$ were dissolved in pyridine $(100 \mathrm{ml})$ and the reaction was carried out at $40^{\circ} \mathrm{C}$ for 24 hours. The mixture was then precipitated in diethyl ether, dissolved in toluene and reprecipitated in diethyl ether. The product was then dried under vacuum.

PentaPZ end group modification (Scheme 3). The transformation of bromine end functional PentaPZ into azide end-functional PentaPZ was adopted from literature. ${ }^{23}$ In short, the bromine end functionalized pentablock $\left(15,100 \mathrm{~g} . \mathrm{mol}^{-1}, 5.4 \mathrm{~g}, 0.36 \mathrm{mmol}\right)$, sodium azide $(234 \mathrm{mg}, 3.6 \mathrm{mmol})$ and dimethyl formamide $(15 \mathrm{ml})$ were added in a flask and stirred well. The mixture was reacted for 24 hours at $50^{\circ} \mathrm{C}$. After that, the azide functionalized pentablock was precipitated in n-hexane, filtered and dried under reduced pressure. Click coupling was used to convert the azide end-functionalized PentaPZ to carboxyl functionalized end group as reported previously in literature..$^{20,23}$ In a round bottom flask, azide functionalized PentaPZ (1.65g, $0.11 \mathrm{mmol})$, copper bromide $(47 \mathrm{mg}, 0.33 \mathrm{mmol})$ and Nppm-ligand (99 mg, 0.66mmol) were added. After capping the flask with septum, the mixture was purged with argon for a couple of minutes. Degassed THF $(4 \mathrm{ml})$ was then added into the mixture. Pentynoic acid $(64.8 \mathrm{mg}, 0.66 \mathrm{mmol})$ was added through a micro syringe. The mixture was then stirred overnight at room temperature. The carboxyl functionalized PentaPZ was precipitated in n-hexane and filtered and dried under vacuum.

Polymer-peptide conjugate synthesis (Scheme 4). The carboxyl terminated Pluronic or PentaPZ, or the acrylic groups of PentaPAA polymers were esterified with NHS as reported in literature. ${ }^{21}$ To a round bottom flask connected with an argon line and bubbler, 
Pluronic/PentaPZ/PentaPAA (0.128 mmol), $0.0792 \mathrm{~g}$ of N,N'-dicyclohexylcarbodiimide (3 X excess, $0.384 \mathrm{~mol}$ ), $0.0442 \mathrm{~g}$ of N-hydroxysuccinimide ( $3 \mathrm{X}$ excess, $0.384 \mathrm{~mol}$ ), and $8 \mathrm{ml}$ of dichloromethane were added. The reaction was carried out at room temperature for 24 hours. The reaction mixture was then filtered, and precipitated in cold diethyl ether. The NHS attachment was verified by ${ }^{1} \mathrm{HNMR}$ with ester peak around $\sim 2.7 \mathrm{ppm}$.

The NHS functionalized polymer was then conjugated with the hydroxyapatite binding peptide as described in the literature. ${ }^{21} 1.5 \mathrm{~g}$ of NHS-polymer was added to a solution of $12 \mathrm{mg}$ of peptide in $50 \mathrm{ml}$ of PBS (pH 7.4) with stirring at room temperature. After 4 hours, additional $1.5 \mathrm{~g}$ of NHS-polymer was added to the mixture. The reaction was maintained at room temperature for 24 hours. A small amount of the reacted mixture was then lyophilized and the conjugation efficiency was determined using reverse phase high pressure liquid chromatography (RP-HPLC). The rest of the reaction mixture was dialyzed against water using a cellulose ester membrane with a molecular weight cut-off of 3500 (Spectrum Labs) for $48 \mathrm{~h}$ in order to remove the uncoupled peptide. The polymer-peptide solution was then lyophilized and a pure white powder was obtained and the peptide attachment was then qualitatively characterized with ${ }^{1} \mathrm{HNMR}$.

The thermoreversible gelation behavior of the polymers was studied by simple gel inversion. A gradient Reverse Phase HPLC (RP-HPLC) method was used to measure the conjugation efficiency of the peptide to the polymer. A (Schimadzu) C-18 analytical column $(5 \mu \mathrm{m}, 4.6 \mathrm{x} 250 \mathrm{~mm})$ was used. A flow rate of $1 \mathrm{ml} / \mathrm{min}$ was used for the gradient elution with the mobile phases of $0.05 \%$ TFA in acetonitrile (solution A) and $0.065 \%$ TFA in $100 \%$ water (solution B). The mobile phase was run as follows: (1) 5\% A for the first $0.01 \mathrm{~min}$, (2) $25 \%$ for the next $24.99 \mathrm{~min}$, (3) $100 \%$ A for the last $5 \mathrm{~min}$ (total run $30 \mathrm{~min}$ ). The polymer- 
peptide conjugate was injected to the system and the unreacted peptide was detected using the UV detector at $220 \mathrm{~nm}$. The concentrations of the unreacted peptide were then calculated using the calibration curve obtained for the pure peptide using the same method. Amount of conjugated peptide was then found by subtracting the unreacted peptide from the initial added peptide to the polymer-peptide conjugation reaction.

Block copolymer - calcium phosphate nanocomposite synthesis. Calcium phosphate (CaP)-block copolymer nanocomposite hydrogel samples were prepared by collaborator Yusuf Yusufoglu using the following procedure.. First, 1.2g copolymer-peptide conjugate obtained by attaching peptide blocks to Pluronic F127 (designated as Pluronic-Pep) and $1.2 \mathrm{~g}$ of pure Pluronic $\mathrm{F} 127$ were mixed with $5.0 \mathrm{~mL} 4.0 \mathrm{M} \mathrm{Ca}(\mathrm{Cl})_{2}$ solution after which the solution was kept in a refrigerator (at $\mathrm{T} \approx 1{ }^{\circ} \mathrm{C}$ ) for 2 days. Then, $3.0 \mathrm{~mL} 4.0 \mathrm{M}\left(\mathrm{NH}_{4}\right)_{2} \mathrm{HPO}_{4}$ solution was added and kept in refrigerator for another 2 days. Later it was aged at room temperature for two days to form a hydrogel nanocomposite containing approximately 22 wt $\%$ polymer-peptide. The $\mathrm{pH}$ of the hydrogel sample was maintained at $\sim 8$ by adding $6 \mathrm{M}$ $\mathrm{NaOH}$ solution. The sample is referred to as Pluronic-Pep22-8, where the numbers 22 and 8 refer to $\mathrm{wt} \%$ of the polymer-peptide conjugate and $\mathrm{pH}$ of the gel, respectively. Similar procedures were used to prepare other $\mathrm{CaP} /$ hydrogel samples, with polymer templates of triblock Pluronic F127, PentaPAA and PentaPZ with peptide groups attached (designated as Pluronic, PentaPAA-Pep and PentaPZ-Pep respectively). The nanocomposite gels were named as, Pluronic30-8, PentaPAA-Pep30-8 and PentaPZ-Pep45-8 hydrogel samples were prepared, where the first $(30,30,45)$ and second numbers (8) refer to polymer content and $\mathrm{pH}$ of the gel respectively.

Characterization. Calcium phosphate nanocomposite samples were analyzed by various 
characterization methods. Scattering measurements along with XRD, NMR, bright field (BF) transmission electron microscopy (TEM), scanning transmission electron microscopy (STEM) using a high angle annular dark field (HAADF) detector, high resolution transmission electron microscopy (HRTEM), selected-area electron-diffraction (SAED), FTIR and TGA experiments were used to study the ordered polymer based nanocomposite superstructure. These studies were conducted by collaborators Yusuf Yusufoglu and Yanyan Hu. Experimental details of NMR, TEM, FTIR and XRD studies can be found in [Yusuf et al. Journal of Materials Research].

Small angle X-ray scattering (SAXS) experiments were performed at the 12-ID beam line at the Advanced Photon Source in Argonne National Laboratory to elucidate the structure of the polymer-peptide gel nanocomposites. By using $12 \mathrm{keV} \mathrm{X-rays}(\lambda=1.035 \AA)$ the data were collected at $1 \mathrm{~s}$ exposure with a $15 \times 15 \mathrm{~cm}^{2}$ CCD detector at a sample-todetector distance of $2 \mathrm{~m}$ to measure the scattered intensity and the transmitted intensity was measured using a photodiode buried in the beam stop. The measured $2 \mathrm{D}$ data for the gel samples sandwiched between kapton tapes were corrected, azimuthally averaged and the scattering data was expressed as a function of $Q(4 \pi \sin \theta / \lambda)$, where $2 \theta$ is the scattering angle. To complement the SAXS data that has higher sensitivity for the inorganic phase, small angle neutron scattering (SANS) was used to highlight the structural features of the copolymer phase in the nanocomposite gels as the neutron scattering contrast for the polymer phase, when in a deuterated solvent, is much higher than the inorganic phase. To increase the scattering contrast of the copolymer the gel samples were prepared in $\mathrm{D}_{2} \mathrm{O}$, and the $\mathrm{pH}$ was adjusted to 8 by using $\mathrm{DCl}$ and $\mathrm{NaOD}$. The samples were sealed in quartz cylindrical cells with a $2 \mathrm{~mm}$ path length and the experiments were performed using the time-of-flight SAND 
instrument at the IPNS at the Argonne National laboratory that yielded data in a Q range of 0.008 to $1 \AA^{-1}$ in a single measurement. The scattering data were corrected following the routine procedures and the $I(Q)$ was placed on an absolute scale in the units of $\mathrm{cm}^{-1}$.

\subsection{Results and Discussion}

Polymer characterization. The polymer-peptide attachment was qualitatively characterized using ${ }^{1} \mathrm{H}$ solution NMR. Compared to the spectra of the polymer alone, the additional peaks between 1-2.5 ppm corresponding to the peptide protons compared to the polymer alone ${ }^{1} \mathrm{H}$ NMR spectra confirmed the conjugation of the peptide to the polymer. The conjugation efficiency was determined by subtracting the amount of unreacted peptide measured by RPHPLC and UV detection from that of the initial amount of peptide used for the conjugation. In the case of Pluronic, the conjugation efficiency was found to be around $19.8 \%$ while it was found to be around $26.6 \%$ and $22.9 \%$ for PentaPAA and PentaPZ respectively.

Structure and Composition of the Inorganic Phase. X-ray diffraction and FTIR measurements were performed to elucidate the inorganic phase and composition of the nanocomposite gels. XRD analysis was done under three different conditions, as prepared nanocomposites, dried nanocomposites, and washed samples where the polymer is removed. All samples mainly exhibit HAp reflections, although some HAp peaks were absent in the vacuum dried samples, may be due to the preferential orientation of HAp crystallites in the polymer matrix. The formation of HAp in the nanocomposites was further confirmed by FTIR analysis from the presence of major phosphate peaks. The details of the XRD and FTIR analysis could be found in [Yusuf et al. Journal of Materials Research]. The evidence of hydroxyapatite phase is further confirmed by direct polarization (DP) and cross-polarization (CP) ${ }^{31} \mathrm{P}$ NMR analysis. These assignments are confirmed by the proton peak positions in $2 \mathrm{D}$ 
${ }^{1} \mathrm{H}-{ }^{31} \mathrm{P}$ correlation spectra without spin diffusion [Yusuf et al. Journal of Materials Research]. The hydroxyapatite fraction in the nanocomposite was determined by thermogravimetric analysis. The analysis showed significant inorganic fractions, approximately $45 \mathrm{wt} \%$ for Pluronic-Pep22-8, $40 \mathrm{wt} \%$ for PentaPAA-Pep30-8, $35 \mathrm{wt} \%$ for Pluronic $30-8$ and $30 \mathrm{wt} \%$ for PentaPZ-Pep45-8. In general, the inorganic content in the present study is significantly larger than the $6-15 \mathrm{wt} \%$ observed in our previous studies of calcium phosphate formation on cationic and polar templates ${ }^{16}$ without hydroxyapatite-binding peptides.

NMR evidence of the nanocomposite formation. Two-dimensional ${ }^{1} \mathrm{H}-{ }^{31} \mathrm{P}$ heteronuclear correlation NMR experiments with ${ }^{1} \mathrm{H}$ spin diffusion,conducted by Yanyan Hu were used to prove the formation of nanocomposites. All the nanocomposites, PentaPAA-Pep30-8, PentaPZ-Pep45-8 and Pluronic30-8, Pluronic-Pep22-8 showed the presence of cross peaks between polymer protons and inorganic phosphate within a few tens of milliseconds confirming the close contact between organic and inorganic phases and nanocomposite formation [Yusuf et al. Journal of Materials Research].

Nanostructures of organic and inorganic phases from SAXS/SANS. By virtue of drastically different interaction of neutrons and X-rays with matter SANS and SAXS serve as excellent complementary tools to investigate the nanostructural features in multicomponent hybrid polymer/inorganic nanocomposites. The complementarity can be clearly seen in Figure 6.1 wherein the SANS and SAXS profiles of the neat and corresponding nanocomposite gels are quite different. While the SANS data of both the polymer and the nanocomposite gel samples look similar and exhibit peaks, the SAXS data show peaks only for the neat polymer, but power-law scattering for the nanocomposites due to the major contribution of the inorganic phase in the latter. The reason for this is that the SAXS signals 
of nanocomposites are over 3 orders of magnitude higher than the neat polymers. In the SAXS data we observe signatures for the average size of the inorganic nanocrystals in the high $\mathrm{Q}$ region and power law scattering in the low $\mathrm{Q}$ region representing their connectivity and correlations in the polymer matrix. In general, the power-law exponent in the low $\mathrm{Q}$ region suggests the connectivity between the fundamental particles whose size can be derived from the scattering intensity in the high $\mathrm{Q}$ region. The absolute value of the power-law exponent in the low Q region will assume values in the vicinity of 1, 2 and 3 for the 1D (rod), $2 \mathrm{D}$ (sheet) and 3D morphologies at $\mathrm{Q} \ell>>1$, where $\ell$ is the length scale of the whole particle. In the case of mass fractal objects the absolute value of the power-law exponent becomes a non-integer in the range of 1 to 3 , the larger the exponent value the denser the distribution of the fundamental particles in the matrix. Although real space images would aid the proper interpretation of the small angle scattering data, modified Guinier analyses for the crosssectional dimensions help to resolve between a mass fractal object versus a rod when the power-law exponent has a value in the vicinity of 1 and a sheet when it has a value around 2. We will use these ideas in the interpretation of the SAXS data below.

For the neat Pluronic gel both the SANS (Fig. 6.1a) and the SAXS (Fig. 6.1b) data show diffraction peaks at $\mathrm{Q} / \mathrm{Q}^{*}=\sqrt{3}: 2: \sqrt{8}$ corresponding to the FCC packing of spherical micelles. The presence of peaks in the SANS data at similar repeat distances for the gels of the neat copolymers and the corresponding nanocomposites indicate that the growth of the inorganic phase has little effect on the long range order and morphologies of the polymer phase. This is consistent with the fact that crystals that nucleate in the interstitial regions will grow around the polymer phase and get connected. The SANS data of the Pluronic30-8 and Pluronic-Pep22-8 nanocomposites clearly show power-law scattering in the low Q regions 
due to the presence of inorganic structures in addition to the peaks associated with the polymer morphology. For the PentaPAA system both the neat polymer and the nanocomposite gels (Figure 6.1c) the SANS data show the BCC ordering with a d-spacing around $16 \mathrm{~nm}$ and the nanocomposite exhibits a power-law scattering in a small low $\mathrm{Q}$ region. The SANS data for the PentaPZ-Pep samples indicate a HCPC structure corresponding to the diffraction peaks at $\mathrm{Q} / \mathrm{Q}^{*}=1: \sqrt{3}: \sqrt{7}: \sqrt{12}$ for the neat polymer as well as the nanocomposite samples, with the d-spacing of $20 \mathrm{~nm}$. In this case, the upturn in the low $\mathrm{Q}$ region was less pronounced when compared to the other composites.

Based on its high sensitivity for the inorganic phase we use SAXS data to evaluate its nanostructural features in the nanocomposites. In the case of Pluronic30-8 (Fig. 6.1b) the length scale $\left(\ell\right.$ ) of the inorganic phase is $\sim 4.86 \mathrm{~nm}$ and a $\mathrm{Q}^{-2.183}$ power-law scattering in the low Q region. On the other hand, for the Pluronic-Pep22-8 gel the length scale of the inorganic phase is $\sim 3.26 \mathrm{~nm}$ that is much smaller and a $\mathrm{Q}^{-1.926}$ power-law scattering in the low Q region. The power-law scattering in the vicinity of 2 implies that the whole particle has a sheet-like morphology that is consistent with the TEM of the nanocomposites presented later. Although we cannot determine the largest dimension of the sheet-like objects with the limited Q range of the small angle scattering data we can use the modified Guinier analysis for a sheet-like morphology by plotting $\ln \left(\mathrm{Q}^{2}\right.$.I) vs. $\mathrm{Q}^{2}$ and fitting a straight line in a $\mathrm{Q}$ region where $\mathrm{Q}_{\max } \mathrm{T}<0.28$, where $\mathrm{T}$ is the average thickness of the sheet-like particles. The thickness of the sheet-like objects in Pluronic30-8 and Pluronic-Pep22-8 gels falls in the range of 4 to $5 \mathrm{~nm}$.

For a given block copolymer, we also observe that the nanocomposite with the peptides exhibit higher scattering intensity than that without the peptide, suggesting that the 
peptide may have some influence in significantly enhancing the growth and nucleation of the inorganic during the nanocomposite formation. These results are consistent with the higher inorganic content measured for the peptide containing nanocomposites.

Figure 6.1d shows the SAXS data for the neat PentaPAA-peptide and the corresponding nanocomposite. Consistent to the SANS data, the neat polymer shows several peaks for a highly ordered $\mathrm{BCC}$ phase with diffraction peaks at $\mathrm{Q} / \mathrm{Q}^{*}=1: \sqrt{2}: \sqrt{3}: 2$, while the nanocomposite exhibits the presence of nanocrystals with an average $\ell \sim 3 \mathrm{~nm}$ and a $\mathrm{Q}^{-}$ 2.565 power law scattering.

In contrast to the observations for the Pluronic and PentaPAA systems, the neat PentaPZ gel (Fig. 6.1f) shows only weak peaks in the SAXS data. This discrepancy could be due to the potential for the samples on kapton tape to dry during the SAXS measurement while the samples for the SANS measurement were contained in sealed quartz cells.

The PentaPZ-Pep45-8 nanocomposite shows the presence of nanocrystals with an average $\ell$ $\sim 3.6 \mathrm{~nm}$ and a $\mathrm{Q}^{-2.165}$ power-law scattering in the low $\mathrm{Q}$ region. Modified Guinier analysis of these nanocomposites revealed a thickness in the range of $4 \mathrm{~nm}$.

In summary, SANS and SAXS data clearly show that both the polymer and the nanocomposite structures have long-range order with nanometer-size morphology. This is in good agreement with the NMR data, where it was found that the polymer and inorganic are in close contact and in the nanometer size range. Furthermore, the conjugated peptide with the polymer does significantly enhance the growth and nucleation of the inorganic compared to the polymer templates without the peptide. 


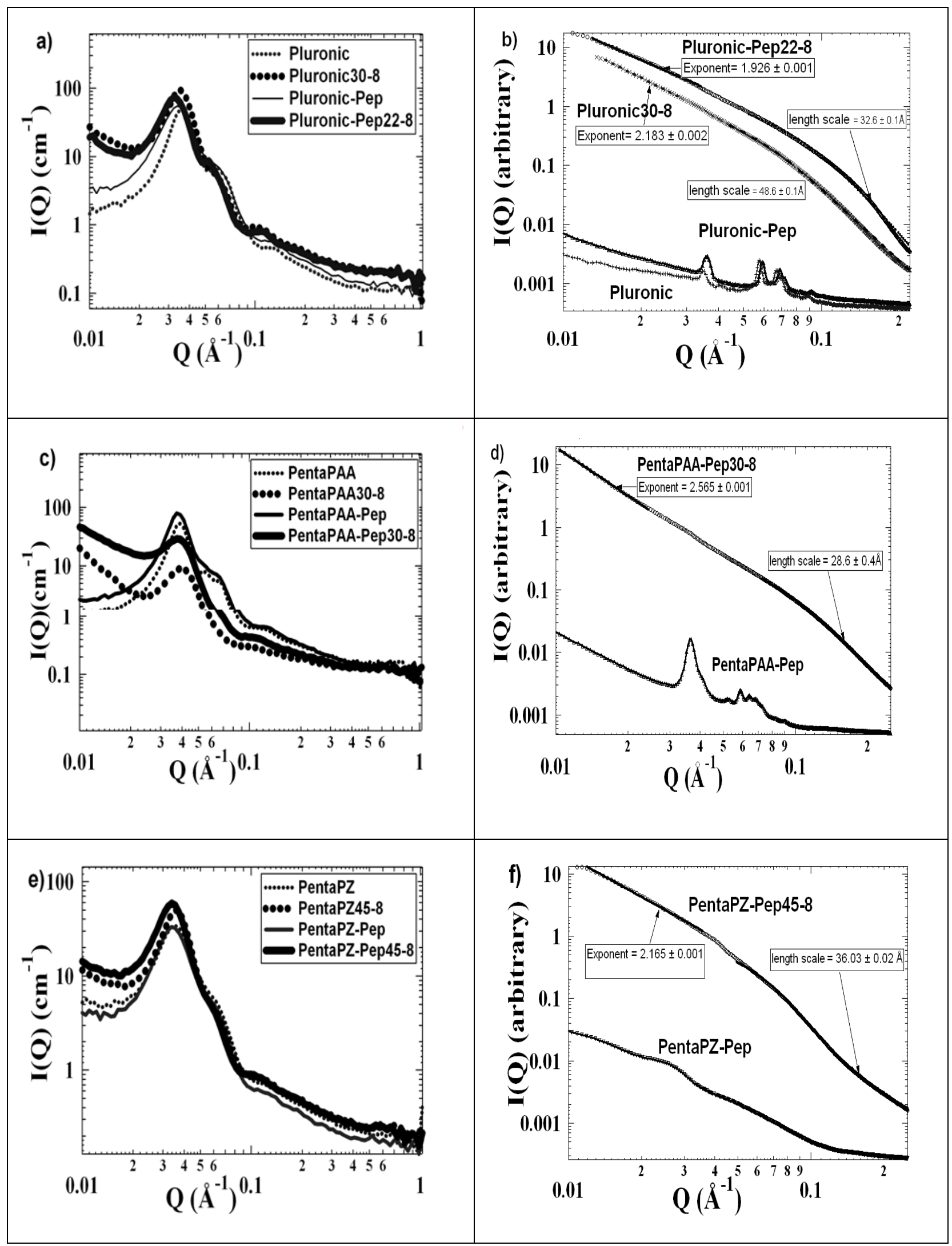


Figure 6.1. a) SANS and b) SAXS of gels of neat Pluronic, Pluronic-Pep and corresponding nanocomposites at $\mathrm{pH} 8.0$; c) SANS and d)) SAXS of gels of neat PentaPAA-Pep and corresponding nanocomposite; e) SANS and f) SAXS of the gels of PentaPZ-Pep and corresponding inorganic nanocomposite. The high Q regions of the SAXS data were fitted using a combined equation consisting of an exponential and a power-law and the low $\mathrm{Q}$ data were fitted using a power-law.

Figure 6.2 illustrates the Scanning transmission electron micrographs (STEM) of 5

wt\% Pluronic, Pluronic-Pep, PentaPAA-Pep and PentaPZ-Pep prepared in deionized water.
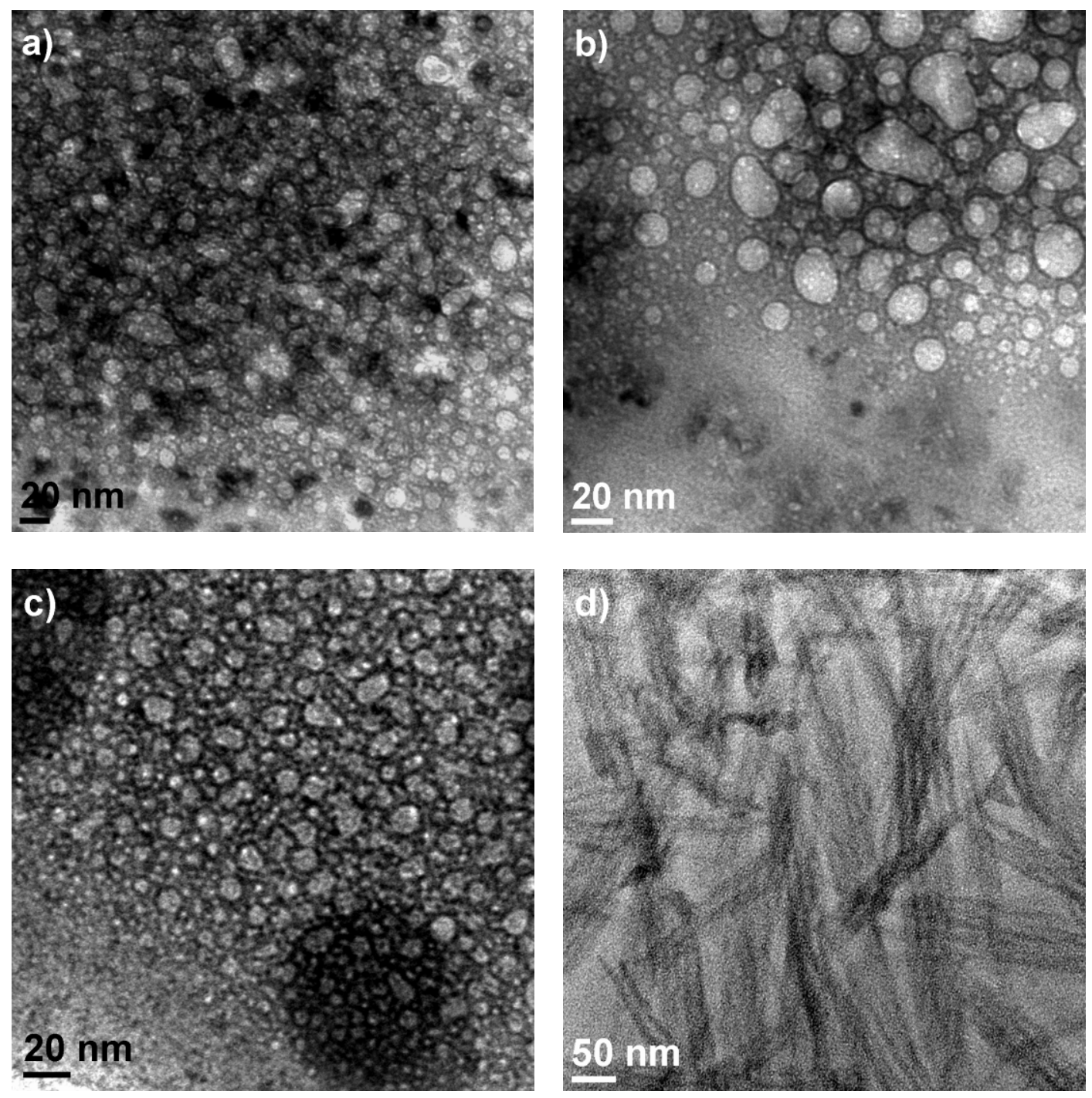

Figure 6.2. STEM/HAADF micrographs of $5 \mathrm{wt} \%$ of polymer solutions prepared in deionized water and stained; a) Pluronic, b) Pluronic-Pep, c) PentaPAA-Pep and d) PentaPZPep. [Adapted from Yusuf et al., Journal of Materials Research]. 
The PentaPZ-Pep polymer solution revealed rod-like structures while all other polymers showed spherical micelles, in agreement with the SAXS and SANS results. The electron beam damage of the polymer precludes the size determination of the spherical micelles by STEM. However, earlier SAXS and SANS revealed that the micelles were approximately $15 \mathrm{~nm}$ in diameter, which is also consistent with our previous studies. ${ }^{16}$

Extensive TEM studies were performed by Yusuf Yusuofglu on the HAp nanocomposite samples to identify the phase and morphology of the calcium phosphate. Pluronic30-8 nanocomposite is constituted of thin elongated plate-like crystals with a mean length and width of about 110 and $20 \mathrm{~nm}$. The morphology of Pluronic-Pep22-8 nanocomposite is quite similar to that observed for the Pluronic30-8. The variable morphology of the nanocrystals is difficult to describe and they vary from $\sim 30-80 \mathrm{~nm}$ in length and $\sim 10-25 \mathrm{~nm}$ in width. HAp nanocrystals of PentaPAA-Pep30-8 are also of platelike shape but with shorter mean length and irregular edges. Morphology of HAp nanocrystals in PentaPZ-Pep45-8, is also observed to be similar to other HAp nanocomposite samples. In general, the morphology of HAp nanocomposites observed in the present study is quite similar to those of various other HAp nanocomposites seen in the literature. ${ }^{24,25}$ However, the morphology of the HAp crystals synthesized without any polymer looked significantly different than the one observed for HAp/hydrogel nanocomposite samples. HAp nanocrystals in polymer-free sample resulted in bulk precipitation of plate-like crystals. It appears that in the presence of the polymers, the morphology of HAp nanocrystals is more elongated plate-like than the one without any polymer. Detailed analysis of the nanocomposite morphology is described in Yusuf el al., Journal of Materials Research.

\subsection{Conclusions}


The summary of the results of this study is tabulated below (Table 6.1). The structural properties of the apatite crystals obtained from this synthesis strategy were quite similar to the apatite present in the bone, with the main mineral phase being nano-sized carbonated HAp which is the main component of bone mineral. ${ }^{26,27}$ Electron micrographs showed that the apatite in bone and in the synthetic nanocomposites appears as plate-like crystals with average dimensions of $\sim 50 \mathrm{~nm}$ in length, $\sim 25 \mathrm{~nm}$ in width, and $2-5 \mathrm{~nm}$ in thickness, and with strong preferred orientation in their c-crystallographic (002) axis. SAXS confirmed the formation of thin, elongated crystallites. Solid-state NMR proved the formation of nanocomposites of this synthesized materials. TGA measurements show that the inorganic content in the nanocomposites was much higher than in those synthesized without the hydroxyapatite nucleating peptides ${ }^{19}$ although it is still lower compared to the natural bone..

Table 6.1. Summary of characterization studies of the biomimetic hydrogel/CaP nanocomposites.

\begin{tabular}{|l|l|l|l|l|l|l|}
\hline \multirow{2}{*}{ Samples } & XRD & $\begin{array}{l}\text { XRD, } \\
\text { FTIR }\end{array}$ & NMR & $\begin{array}{l}\text { STEM, } \\
\text { HRTEM, } \\
\text { SAED }\end{array}$ & $\begin{array}{l}\text { SAXS, } \\
\text { SANS }\end{array}$ & TGA \\
\cline { 2 - 7 } & Gel & $\begin{array}{l}\text { Vacuum } \\
\text { dried }\end{array}$ & $\begin{array}{l}\text { Vacuum } \\
\text { dried }\end{array}$ & $\begin{array}{l}\text { Gel diluted } \\
\text { to } 5 \text { t } \% \\
\text { Polymer }\end{array}$ & $\begin{array}{l}\text { Gel } \\
\text { morphology }\end{array}$ & Dried \\
\hline Pluronic30-8 & HAp & HAp & HAp & $\begin{array}{l}\text { Elongated } \\
\text { plate-like } \\
\text { HAp }\end{array}$ & FCC & $35 \mathrm{wt} \% \mathrm{CaP}$ \\
\hline $\begin{array}{l}\text { Pluronic- } \\
\text { Pep22-8 }\end{array}$ & HAp & HAp & HAp & $\begin{array}{l}\text { Elongated } \\
\text { plate-like } \\
\text { HAp }\end{array}$ & FCC & $45 \mathrm{wt} \% \mathrm{CaP}$ \\
\hline $\begin{array}{l}\text { PentaPAA- } \\
\text { Pep30-8 }\end{array}$ & HAp & HAp & HAp & $\begin{array}{l}\text { Elongated } \\
\text { plate-like } \\
\text { HAp }\end{array}$ & BCC & $40 \mathrm{wt} \% \mathrm{CaP}$ \\
\hline $\begin{array}{l}\text { PentaPZ- } \\
\text { Pep45-8 }\end{array}$ & $\begin{array}{l}\text { HAp/ } \\
\text { Brushite }\end{array}$ & HAp & $\begin{array}{l}\text { HAp/ } \\
\text { Brushite }\end{array}$ & $\begin{array}{l}\text { Elongated } \\
\text { plate-like } \\
\text { HAp }\end{array}$ & HCPC & $30 \mathrm{wt} \% \mathrm{CaP}$ \\
\hline
\end{tabular}


In summary, we have successfully developed biomimetic polymer-hydroxyapatite nanocomposites using hydroxyapatite nucleating peptides conjugated to self-assembling block copolymers from a bottom-up approach. The thermoreversible gelation properties of these nanocomposites synthesized by a biomineralization approach, could potentially enable them to serve as injectable biomaterials for bone/cartilage tissue repair.

\subsection{Acknowledgements}

This work was supported by the U.S. Department of Energy under contract number DEAC02-07CH11358. This work benefited from the use of APS and the IPNS, funded by the U.S. DOE, Office of Science, Office of Basic Energy Science under contract no DE-AC02$06 \mathrm{CH} 11357$.

\subsection{References}

1. J. Aizenberg: Crystallization in patterns: A bio-inspired approach. Adv. Mater. 16, 1295-1302 (2004).

2. J.L. Arias, A. Neira-Carrillo, J.I. Arias, C. Escobar, M. Bodero, M. David, and M.S. Fernandez: Sulfated polymers in biological mineralization: A plausible source for bio-inspired engineering. J. Mater. Chem. 14, 2154-2160 (2004).

3. B.L. Smith, G.T. Paloczi, P.K. Hansma, and R.P. Levine: Discerning Nature's mechanism for making complex biocomposite crystals. J. Crystal Growth 211, 116121 (2000).

4. Z.Y. Tang, N.A. Kotov, S. Magonov, and B. Ozturk: Nanostructured artificial nacre. Nat. Mater. 2, 413-U8 (2003).

5. M. Aizawa, H. Ueno, K. Itatani, and I. Okada: Synthesis of calcium deficient apatite fibers by a homogeneous precipitation metho and their characterization. J. Eur. Cer. Soc. 26, 501-507 (2006).

6. E.G. Bellomo and T. Deming: Monoliths of aligned silica-polypeptide hexagonal platelets. JACS 128, 2276-79 (2006).

7. J.N. Cha, G.D. Stucky, D.E. Morse, and T.J. Deming: Biomimetic synthesis of ordered silica structures mediated by block copolypeptides. Nature 403, 289-292 (2000).

8. G. Falini, M. Gazzano, and A. Ripamonti: Control of architectural assembly of octacalcium phosphate crystals in denatured collageneous matrices. J. Mater. Chem. $10,535-538$ (2000). 
9. J.L. Sumerel, W. Yang, D. Kisailus, J.C. Weaver, J.H. Choi, and D.E. Morse: Biocatalytically templated synthesis of titanium dioxide. Chem. Mater. 15, 4804-09 (2003).

10. V. Bansal, D. Rautaray, A. Bharde, K. Ahire, A. Sanyal, A. Ahmad, and M. Sastry: Fungus mediated biosynthesis of silica and titania particles. J. Mater. Chem. 15, 2583-2589 (2005).

11. V.M. Rusu, N. C.H., M. Wilke, B. Tiersch, P. Fratzl, and M.G. Peter: Size controlled hydroxyapatite nanoparticles as self-organized organic-inorganic composite materials. Biomaterials 26, 5414-5426 (2005).

12. J. Song, V. Malathong, and C.R. Bertozzi: A bottom-up approach for the development of artificial bone. JACS 127, 3366-3372 (2005).

13. J. Song, E. Saiz, and C.R. Bertozzi: An efficient process towards 3-dimensional bonelike composites. JACS 125, 1236-43 (2003).

14. J. Song, E. Saiz, and C.R. Bertozzi: Preparation of PHEMA-CP composites with high interfacial adhesion via template driven mineralization. J. Eur. Cer. Soc. 23, $2905-$ 2919 (2003).

15. G. Xu, I. Aksay, and J.T. Groves: Continuous crystalline carbonate apatite thin films. JACS 123, 2196-2203 (2001).

16. D. Enlow, A. Rawal, M. Kanapathipillai, K. Schmidt-Rohr, S. Mallapragada, C.T. Lo, P. Thiyagarajan, and M. Akinc: Synthesis and characterization of self-assembled block copolymer templated calcium phosphate nanocomposite gels. J. Mater. Chem. 17, 1570-1578 (2007).

17. S. Chang, H. Chen, J. Liu, D. Wood, P. Bentley, and B. Clarkson: Synthesis of a potentially bioactive hydroxyapatite nucleating molecule. Calc. Tiss. Int. 78, (2006).

18. M. Determan, S. Seifert, P. Thiyagarajan, and S.K. Mallapragada: Synthesis and characterization of temperature and ph-sensitive self assembling pentablock copolymers. Polymer 46, 6933-46 (2005).

19. M. Kanapathipillai, Y. Yusufoglu, A. Rawal, Y. Hu, C.T. Lo, P. Thiyagarajan, Y. Kalay, M.A. Akinc, S.K. Mallapragada, and K. Schmidt-Rohr: Ionic block copolymer template directed calcium phosphate nanocomposites. Chem. Mater. Submitted, (2008).

20. J.-F. Lutz, H.G. Boerner, and K. Weichenhan: Combining atom transfer radical polymerization and click chemistry: A versatile method for the preparation of endfunctional polymers. Macromol. Rapid Comm. 26, 514-518 (2005).

21. F. Zeng, H. Lee, and C. Allen: Epidermal growth factor-conjugated poly(ethylene glycol)-block- poly(d-valerolactone) copolymer micelles for targeted delivery of chemotherapeutics. Bioconj. Chem. 17, 399-409 (2006).

22. D. Bali, L. King, and S. Kim: Syntheses of new gramicidin a derivatives. Austr. J. of Chem. 56, 293-300 (2003).

23. V. Coessens, Y. Nakagawa, and K. Matyjaszewski: Synthesis of azido endfunctionalized polyacrylates via atom-transfer radical polymerization. Polymer Bull. 40, 135-142 (1998).

24. Z. Li, Y. Li, A. Yang, X. Peng, X. Wang, and Z. Xiang: Preparation and in vitro investigation of chitosan/nano-hydroxyapatite composite used as bone substitute materials. J. Mater. Sci.: Mater. in Med. 16, 213-219 (2005). 
25. C. Lai, S. Tang, Y. Wang, and K. Wei: Formation of calcium phosphate nanoparticles in reverse microemulsions. Mater. Lett. 59, 210-214 (2004).

26. M. Vallet-Regi and J.M. Gonzalez-Calbet: Calcium phosphates as substitution of bone tissues. Prog. in Solid State Chem. 32, 1-31 (2004).

27. X. Su, K. Sun, F.Z. Cui, and W.J. Landis: Organization of apatite crystals in human woven bone. Bone 32, 150-162 (2003) 


\title{
CHAPTER 7. STIMULI RESPONSIVE BORONIC ACID BASED PENTABLOCK COPOLYMERS FOR GLUCOSE RESPONSIVE APPLICATIONS
}

\author{
A paper submitted to Journal of Biomaterials Science Polymer Edition \\ M. Kanapathipillai ${ }^{\text {a }}$ Surya. K. Mallapragada*
}

\subsection{Abstract}

A novel pentablock copolymer with glucose responsiveness and thermoreversible gelation properties was synthesized for potential biological applications. The pentablock copolymer consisting of Pluronic ${ }^{\circledR}$ triblock and poly(3-acryl-amidophenylboronic acid) (PAAPBA) side blocks was synthesized via reversible addition fragmentation transfer (RAFT) polymerization. The polymer is capable of forming temperature responsive hydrogels at physiological temperatures due to the hydrophobic polypropylene oxide (PPO) block in the Pluronic ${ }^{\circledR}$ triblock, and can be used as an injectable drug delivery system. The APBA monomer is an anionic monomer with a $\mathrm{pKa} \sim 8.3$. Glucose forms a charged complex with the phenyl boronic acid moiety of the APBA and results in glucose responsive solubility in aqueous solutions. Titration measurements showed that the pKa value of the pentablock copolymer was reduced in the presence of glucose. Laser light scattering experiments were used to demonstrate the glucose responsive micellization properties of this copolymer, where a significant reduction in micellar size was observed in the presence of glucose. Further, the polymer gels exhibited swelling in the presence of glucose, which resulted in lower moduli values for the gels, as observed from rheological measurements. These stimuli responsive pentablock copolymers can be of importance in various glucose responsive applications, such as drug delivery. 
${ }^{a}$ Department of Chemical Engineering, Iowa State University and Ames Laboratory, 144 Spedding Hall, Ames, IA 50011, USA. E-mail address: umai@iastate.edu

*Corresponding author. Department of Chemical Engineering, Iowa State University and Ames Laboratory, 144 Spedding Hall, Ames, IA 50011, USA Phone: +1 5152947407 Fax: +15152942689 E-mail address: suryakm@iastate.edu

\subsection{Introduction}

Stimuli-responsive polymers exhibit large, sharp changes in response to physical stimuli (such as temperature, solvents, or light) or to chemical stimuli. Changes in the physical, chemical or biochemical environments of these polymers results in modulation of the solubility or chain conformation of the polymer [1-3]. They have been widely investigated for the applications in biomedical and materials research such as in biomimetic actuators, drug delivery systems and bioseparations [1].

Recently, water-soluble glucose-sensitive boronic acid containing polymers have shown great potential in biological applications [4-7]. Phenylboronic acid is a molecular recognition ligand, which is widely used in affinity separation and chromatographic studies $[8,9]$. Phenylboronic acid compounds are in equilibrium between uncharged and charged forms in aqueous medium. Through complexation with glucose, the equilibrium shifts in the direction of increasingly charged phenylborates as only charged borates can form a stable complex with glucose in aqueous media $[9,10]$. Based on this principle, many research studies have investigated the use of boronic acid based block copolymers for applications such as glucose-sensitive insulin delivery systems, glucose sensing and molecular recognition separation techniques $[4,5,9,10]$.

A block copolymer containing poly(3-acrylamidophenylboronic acid) and poly (dimethylaminoethyl methacrylate), poly (AAPBA)-b-poly(DMAEMA) showed glucose sensitive responsiveness due to the protonation of AAPBA in the presence of glucose [4]. 
Since in aqueous medium these AAPBA compounds are in equilibrium as charged and uncharged forms, the complexation of glucose is able to shift the equilibrium towards the direction of increased charge of phenylborates, since only charged borates can form complexes with glucose in aqueous media. Several studies have been done on glucoseresponsive crosslinked gels using proteins such as the enzyme glucose oxidase (GOD) [11] or lectin concanavalin A (Con A) [12] as well as synthetic moieties such as phenylboronic acid (PBA) $[4,5]$. Although the synthetic moieties are less specific, they have the advantage of greater reliability and long-term stability and do not suffer from drawbacks associated with possible protein denaturation. Hence it represents the characteristics to induce gel swelling upon glucose complexation. From this basis, Kataoka et al., have developed gels with on-off regulation of insulin release, initially operating at a $\mathrm{pH}$ of 9 [13] and recently at physiological $\mathrm{pH}$ conditions by modifying the chemical structure of the receptor with an electron-drawing group in the phenyl ring $[9,10]$.

Although the polymer gel systems reported above are capable of exhibiting glucose responsive properties and hence are potential drug delivery devices for insulin delivery, due to the crosslinked nature of these gels, it is difficult to implant and remove them from the body. Therefore, our work is focused on novel injectable boronic acid based gel systems that can form glucose-responsive physical gels in vivo at physiological temperatures thereby eliminating difficulties associated with implanting and removing the gels. Recently, we have demonstrated that a pH-sensitive Pluronic ${ }^{\circledR}$ based pentablock copolymer is capable of forming hydrogels and can be used as potential stimuli-responsive injectable drug depot system $[14,15]$. In this study, we are focusing on a glucose-responsive boronic acid-based 
pentablock copolymers that can undergo thermoreversible gelation and hence could be used in future drug delivery applications.

Several polymerization techniques have been employed in the synthesis of polymers with boronic acid moieties, in which conventional radical polymerization has proven to be the most efficient of all [16-18]. As far as acrylamido hydrophilic block copolymers are concerned, although atom transfer radical polymerization (ATRP) has been used recently, reversible addition fragmentation chains transfer polymerization (RAFT), showed excellent results [18-20]. Although several works were reported on the uncontrolled random copolymerization of acrylamido and boron containing monomers [13], recently Cambre et. al. have demonstrated the use of RAFT polymerization in the synthesis of well-defined water-soluble acrylamido and boron containing block copolymers [18].

We have synthesized a novel Pluronic ${ }^{\circledR}$ (F127) based pentablock copolymer with PAAPBA blocks as a glucose-sensitive moiety using RAFT polymerization. The pentablock copolymer is capable of exhibiting thermoreversible gelation due to the Pluronic ${ }^{\circledR}$ triblock. The focus of this study is to develop a pentablock copolymer that can exhibit glucose- and thermoresponsive properties through a facile RAFT synthesis method. The RAFT polymerization was performed with pinacol esterified AAPBA monomer to have an efficient radical transfer, and the deprotection of pinacol ester was achieved by the use of borono polystyrene similar to previously reported methods [21, 22]. PAAPBA exhibits a pKa value of around 8.3 and forms charged borates above this $\mathrm{pH}$. Through complexation with glucose, charged phenylborates attain a stable complex, rendering the polymer more hydrophilic which leads to the swelling of the gel, exhibiting glucose responsiveness $[4,5]$. Further, we have investigated its solution properties such as micelle size, $\mathrm{pKa}$ and gel swelling behavior 
in glucose environment and hence their future potential in glucose responsive applications such as in biosensors and glucose responsive drug delivery.

\subsection{Materials and methods}

Aminophenyl boronic acid (APBA) was purchased from TCI America. [1-ethyl-3(dimethylamino) propoyl] carbo-diimide hydrochloride (EDC) and polystyrene supported boronic acid resin ( $\sim 3 \mathrm{mmol} / \mathrm{g})$ were purchased from Alfa Aesar and used as received. 2,2'Azobisisobutyronitrile (AIBN, Sigma, 98\%) was recrystallized from ethanol. All the other chemicals were purchased from Sigma Aldrich and Fisher Scientific and were of high purity.

\subsubsection{Glucose responsive pentablock copolymer synthesis.}

7.3.2. RAFT chain transfer agent synthesis (S-1-dodecyl-S'-( $\alpha, \alpha^{\prime}$-dimethyl- $\alpha$ "-acetic acid) trithiocarbonate.

The synthesis procedure was adopted from Lai et al.[23] method. Briefly, 1dodecanethiol (40.38 g, 0.2 mole), acetone (96.2 g, 1.65 mole), and Aliquot 336 (tricaprylylmethylammonium chloride, $3.25 \mathrm{~g}, 0.008 \mathrm{~mol}$ ) were mixed around $10^{\circ} \mathrm{C}$ under nitrogen atmosphere in a jacketed reactor. After that, $50 \%$ sodium hydroxide $(16.7 \mathrm{~g}, 0.21$ mol) was added slowly. After 15 minutes of additional stirring, carbon disulfide (15.21g, 0.2 mol) in acetone $(20.18 \mathrm{~g}, 0.39 \mathrm{~mol})$ was added over 10 minutes and the color of the solution turn into red at that time. Chloroform ( $35.6 \mathrm{~g}, 0.3 \mathrm{~mol})$ was added after which $50 \%$ of $\mathrm{NaOH}$ solution $(80 \mathrm{~g}, 1 \mathrm{~mol})$ was added dropwise. The mixture was stirred overnight and the following day $300 \mathrm{ml}$ of water was added followed by $50 \mathrm{ml}$ of concentrated $\mathrm{HCl}$ to acidify the mixture. To evaporate the acetone, nitrogen was purged through the reactor. Later, the solid product was filtered out then stirred in $500 \mathrm{ml}$ of 2- propanol. After the stirring, the remaining solid was filtered out and the solution was concentrated to dryness. The resulting 
solid was recrystallized from hexanes and a yellow crystalline solid product was obtained and verified with ${ }^{1} \mathrm{HNMR}$.

\subsubsection{F127 RAFT chain transfer agent synthesis (F127-CTA).}

Equal number of moles of Pluronic ${ }^{\circledR}$ F127 and RAFT-CTA were dissolved in methylene chloride with 4-dimethylaminopyridine (DMAP) $\left(0.2\right.$ eq.) and cooled to $0^{\circ} \mathrm{C}$ after which dicyclohexylcarbodiimide (DCC) solution (1.5 eq.) was added slowly. The reaction was stirred overnight. The resulting solution was then precipitated in diethyl ether, redissolved in THF, centrifuged and filtered and reprecipitated in ether and a pure F127-CTA agent was obtained. The product was then characterized with ${ }^{1} \mathrm{HNMR}$.

7.3.4. Monomer (3-Acrylamideophenylboronic Acid, (AAPBA)) synthesis.

AAPBA was synthesized as previously described by Lapeyre et al.,[5]. Briefly, 1.862 $\mathrm{g}$ (10 mmole) of 3-aminophenylboronic acid was dissolved in $15 \mathrm{ml}$ water. The $\mathrm{pH}$ of the solution was adjusted to 4.8. The solution was then immersed into an ice bath, and $2.3 \mathrm{~g} \mathrm{(12}$ mmole) of EDC was added and the $\mathrm{pH}$ was again adjusted to 4.8. In a separate flask, $0.83 \mathrm{ml}$ (12 mmole) of acrylic acid in a solution of $10 \mathrm{ml}$ water was prepared and the $\mathrm{pH}$ was adjusted to 4.8. The solutions were then mixed together in an inert atmosphere and the reaction was maintained around $15^{\circ} \mathrm{C}$ and allowed to stir overnight at room temperature. The reacted mixture was then filtered, and extracted with ethyl ether. The ether was then rotary evaporated and the crude oil product was then mixed with water and the mixture was stirred under ice and a white solid precipitate was obtained. ${ }^{1} \mathrm{H}$ NMR(APBA) (400 MHz, DMSO): $5.75\left(1 \mathrm{H}, \mathrm{CH}_{2}=\mathrm{CH}-\right), 6.25\left(1 \mathrm{H}, \mathrm{CH}_{2}=\mathrm{CH}-\right), 6.3\left(1 \mathrm{H}, \mathrm{CH}_{2}=\mathrm{CH}-\right), 7.25(1 \mathrm{H}$, phenyl), $7.5(1 \mathrm{H}$, phenyl), $7.8\left(1 \mathrm{H}\right.$, phenyl), $7.85\left(1 \mathrm{H}\right.$, phenyl), $8.0\left(2 \mathrm{H},-\mathrm{B}(\mathrm{OH})_{2}\right), 10.1(1 \mathrm{H}, \mathrm{NH})$.

\subsubsection{Pinacol esterification of AAPBA monomer.}


The monomer was esterified with pinacol similar to the procedure reported previously $[18,21]$. AAPBA (1g, $5.3 \mathrm{mmol})$, pinacol $(700 \mathrm{mg}, 6 \mathrm{mmol})$, were added to dry THF. The mixture was stirred for $18 \mathrm{~h}$. The product was concentrated under vacuum and then precipitated with n-hexane to give the esterified monomer with a yield $\sim 95 \% .{ }^{1} \mathrm{H}-\mathrm{NMR}$ (400 $\left.\mathrm{MHz}, \mathrm{CDCl}_{3}\right)$ revealed the sharp pinacol ester peak at $1.38\left(\mathrm{~s}, 12 \mathrm{H},\left(\left[\mathrm{CH}_{3}\right]_{2} \mathrm{C}-\mathrm{C}-\mathrm{CH}_{3}\right]_{2}\right)$.

\subsubsection{RAFT polymerization of Pinacol esterified AAPBA monomer with F127- CTA.}

The boronic acid pentablock copolymer was synthesized by RAFT polymerization. First, $(200 \mathrm{mg}, 15 \mu \mathrm{mol})$ of CTA RAFT initiator was mixed with $(200 \mathrm{mg}, 735 \mu \mathrm{mol})$ of pinacol esterified AAPBA in dry THF $(2 \mathrm{ml})$ with AIBN catalyst $(\sim 2 \mu \mathrm{mol})$. The reaction was carried out overnight at $70^{\circ} \mathrm{C}$ under inert conditions. The product was then precipitated in ether and dried under vacuum.

\subsubsection{Deprotection of pinacol ester from boronic pentablock copolymer.}

Deprotection of the pinacol ester was carried out as previously reported [22]. The polymer $(0.1 \mathrm{~g}, 0.25 \mathrm{mmol})$ was mixed with an excess amount of (x5) polystyrene-supported boronic acid $(0.55 \mathrm{~g}, \sim 3.0 \mathrm{mmol} / \mathrm{g})$, in a flask containing $10 \mathrm{ml}$ acetonitrile with $10 \% 1 \mathrm{M}$ $\mathrm{HCl}$ solution. The mixture was stirred at room temperature for $18 \mathrm{~h}$. The solution was then filtered to remove the polymer resin and the filtrate was then concentrated by rotary evaporation. The product was then precipitated in ether and a deprotected pentablock copolymer was obtained.

\subsection{Characterization}

7.4.1. Structural investigation with NMR. 
The RAFT synthesis procedure of the boronic acid pentablock copolymer is shown in Figure 7.1. The chemical structures and the molecular weight of the copolymers were found from ${ }^{1} \mathrm{HNMR}$ characterization and end group analysis.

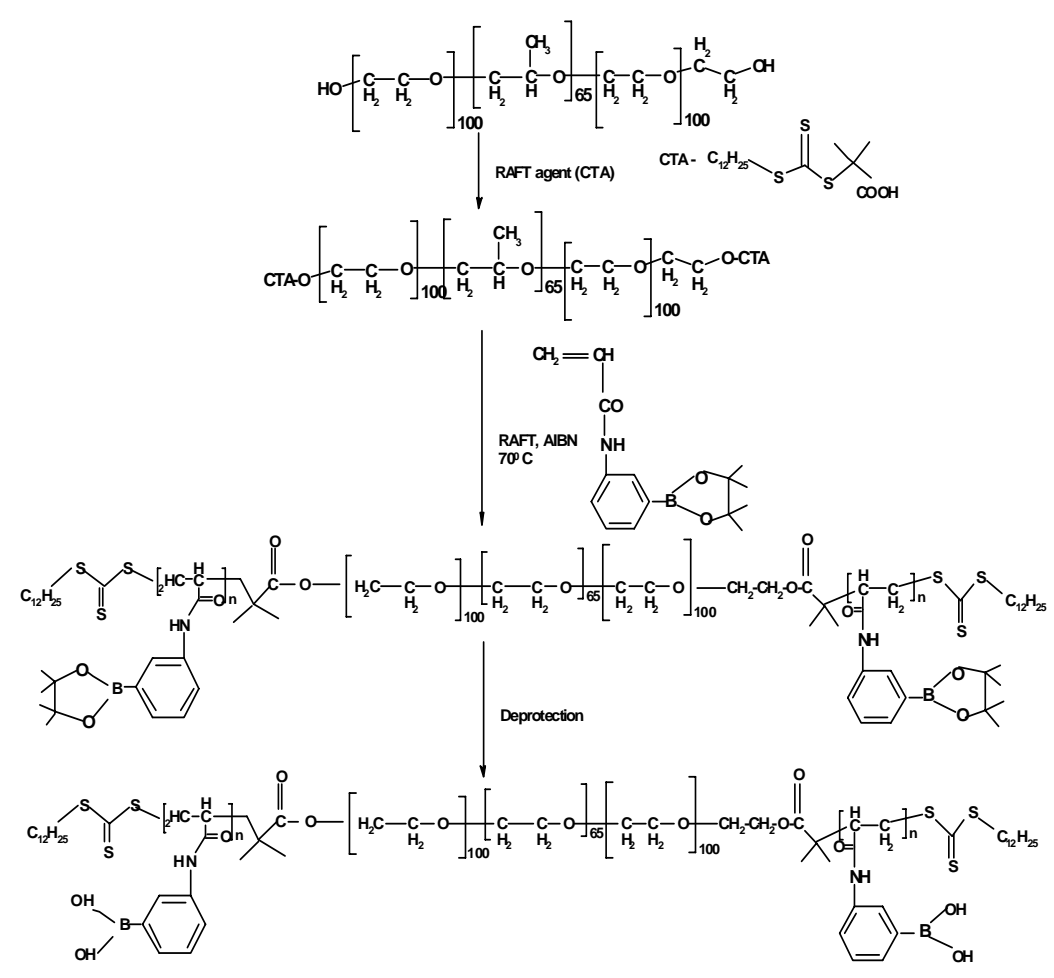

Figure.7.1. Boronic acid pentablock synthesis scheme using RAFT polymerization

\subsubsection{Potentiometric titration.}

Titration measurements were performed to find the dependency of the $\mathrm{pKa}$ value of the polymer with and without different glucose concentrations. Potentiometric titrations were carried out using a corning $313 \mathrm{pH} /$ temperature meter at room temperature. All solutions were prepared with nanopure water. Samples were prepared by dissolving $20 \mathrm{mg}$ of the boronic acid pentablock copolymer in $20 \mathrm{ml}$ water. The pentablock copolymer solution $\mathrm{pH}$ was first raised to $\mathrm{pH} 12$ by adding $1.0 \mathrm{M} \mathrm{NaOH}$ and then back titrated using $1.0 \mathrm{M} \mathrm{HCl}$. The decrease in ionization of the boronic group was calculated by subtracting the $\mathrm{H}^{+}$ 
concentration in solution from the total $\mathrm{H}^{+}$added to the boronic acid pentablock copolymer solution according to [24].

\subsubsection{Effects of glucose concentration on boronic acid pentablock copolymer micelles.}

Light scattering technique was applied to determine the size of copolymer micelles in aqueous solutions under different glucose concentration conditions. Measurements were performed using a zeta particle size analyzer (Malvern instruments, U.K.) operating with a $\mathrm{HeNe}$ laser at $90^{\circ}$. Samples were prepared with $4 \mathrm{mg} / \mathrm{ml}$ of boronic acid pentablock copolymer in water at $\mathrm{pH} 8.5$ with and without different amount of glucose concentrations, and were filtered with $0.22 \mu \mathrm{m}$ filters immediately prior to analysis. Data collection and analysis was performed with Malvern instrument software.

\subsubsection{Boronic acid pentablock copolymer gelation and its glucose sensitivity.}

The gelation properties of the copolymers were investigated by simple gel inversion techniques. Boronic acid pentablock copolymer gels with various concentrations were prepared in $\sim \mathrm{pH} 8.5$ aqueous solutions (closer to their pKa value). The polymer gelation conditions were then studied as a function of temperature. Rheological experiments were carried out using an ARES rheometer from TA instruments to investigate the glucose responsive swelling of the boronic acid copolymer gels. Copolymer gels of $40 \mathrm{wt} \%$ with and without $100 \mathrm{mg} / \mathrm{ml}$ glucose concentration was used for the study. A parallel plate with diameters of $50 \mathrm{~mm}$ and gap of $1.0 \mathrm{~mm}$ was used for all samples. The moduli values of physical hydrogels formed from the copolymers were investigated with dynamic strain controlled (50\%) mode at room temperature. Frequency sweeps were carried out with angular frequency varied from $1 \mathrm{rad} / \mathrm{s}$ to $0.001 \mathrm{rad} / \mathrm{s}$ and the moduli measurements were recorded. 


\subsection{Results}

Boronic acid pentablock copolymers were synthesized using RAFT polymerization and characterized to elucidate their glucose-sensitive behavior in aqueous environments. ${ }^{1}$ HNMR measurements were performed to find the composition and molecular weight of the boronic acid pentablock copolymers. Fig. 7.2 shows the ${ }^{1}$ HNMR spectra of F127-RAFT chain transfer agent. RAFT polymerization was performed to polymerize the AAPBA monomer with the F127 RAFT chain transfer agent to obtain the boronic acid pentablock copolymer.

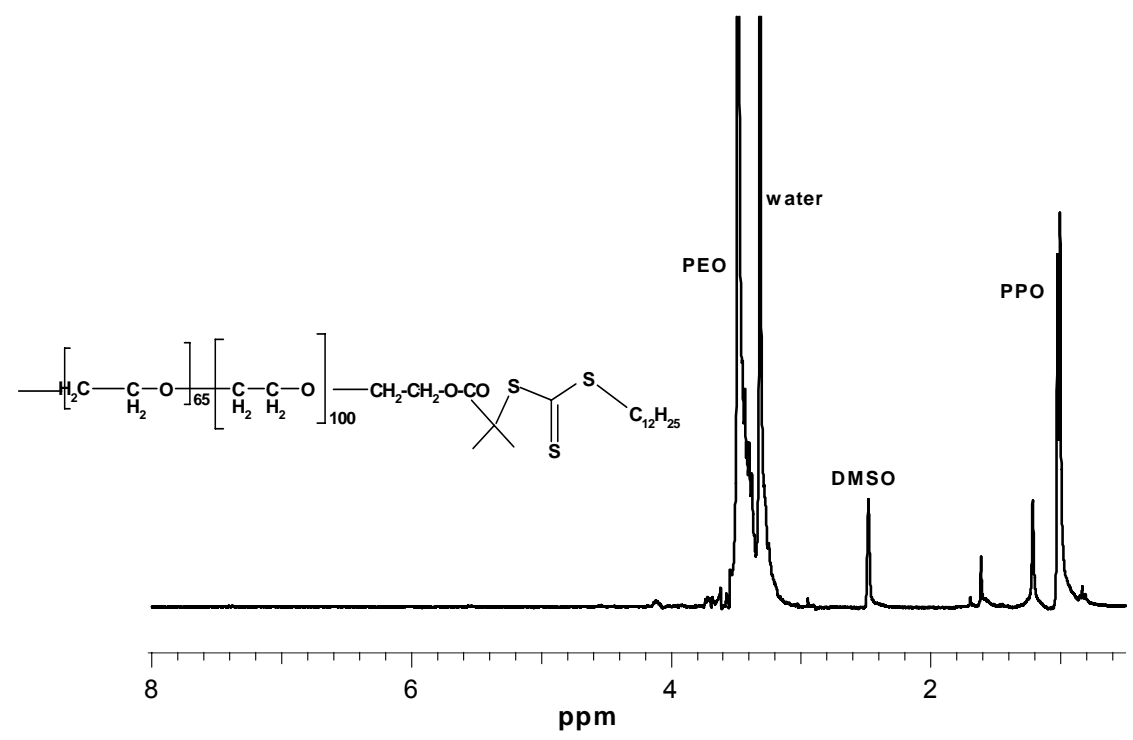

Figure.7.2. ${ }^{1} \mathrm{HNMR}$ spectra of F127-CTA

The pentablock copolymer obtained by the RAFT polymerization from the F127CTA and AAPBA monomer before and after the deprotection of the pinacol ester group was shown in Fig. 7.3. The degree of polymerization of the boronic acid groups to F127 triblock copolymer was found using the end group analysis from the NMR spectra peak area ratios of Pluronic $^{\circledR}$ and boronic acid groups. Four different molecular weight polymers with different boronic acid side blocks were synthesized for the study. The detailed boronic acid side 
blocks and the respective molecular weights and the molar percent of the boronic acid in the copolymers are tabulated in Table 7.1. The polymer nomenclature was chosen to represent the triblock copolymer (F127), and the boronic acid side blocks (BA) and its corresponding number of boronic acid side groups (n).

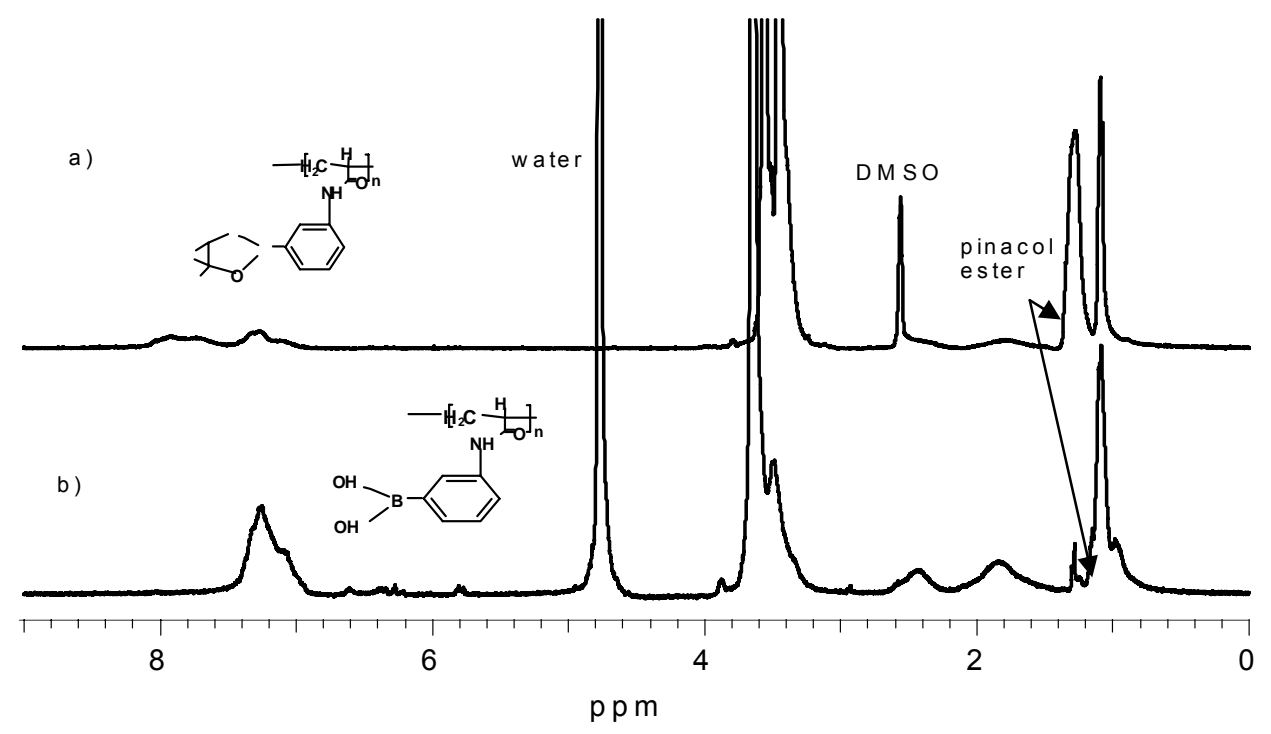

Figure.7.3. An example ${ }^{1} \mathrm{HNMR}$ spectra of a) boronic acid pentablock copolymer in DMSO before deprotection $b$ ) in $\mathrm{D}_{2} \mathrm{O}$ after deprotection

Table 7.1. Composition and molecular weights of boronic acid copolymers

\begin{tabular}{|c|c|c|c|c|}
\hline \multirow{2}{*}{$\begin{array}{l}\text { Polymer } \\
\text { Abbreviation }\end{array}$} & \multicolumn{2}{|c|}{ Feed composition (molar ratio) } & \multirow{2}{*}{$\begin{array}{l}\text { Total number } \\
\text { of borate blocks } \\
\text { in copolymer }\end{array}$} & \multirow{2}{*}{$\begin{array}{c}\text { Molecular } \\
\text { weight from } \\
{ }^{1} \mathrm{HNMR}\end{array}$} \\
\hline & F127-CTA & AAPBA & & \\
\hline F127-BA-6 & 1 & 12 & 12 & 15,404 \\
\hline F127-BA-15 & 1 & 30 & 30 & 18,750 \\
\hline F127-BA-25 & 1 & 50 & 50 & 22,620 \\
\hline F127-BA-50 & 1 & 100 & 100 & 31,782 \\
\hline
\end{tabular}


The copolymers showed thermoreversible gelation depending on the polymer molecular weight, concentration and temperature. A gelation diagram obtained for two different copolymers is shown in Fig. 7.4. When the boronic acid content was increased, the copolymer gels become weak and loosely packed due to the charged borate groups. The copolymers formed gels when the temperature increased due to the LCST behavior of the PPO blocks in the pentablock copolymer. Above a certain temperature, depending on the boronic acid groups in the copolymer, the LCST behavior of the PPO is less favored due to the increased mobility of the borate charged complexation groups resulting in the swelling of the gel.

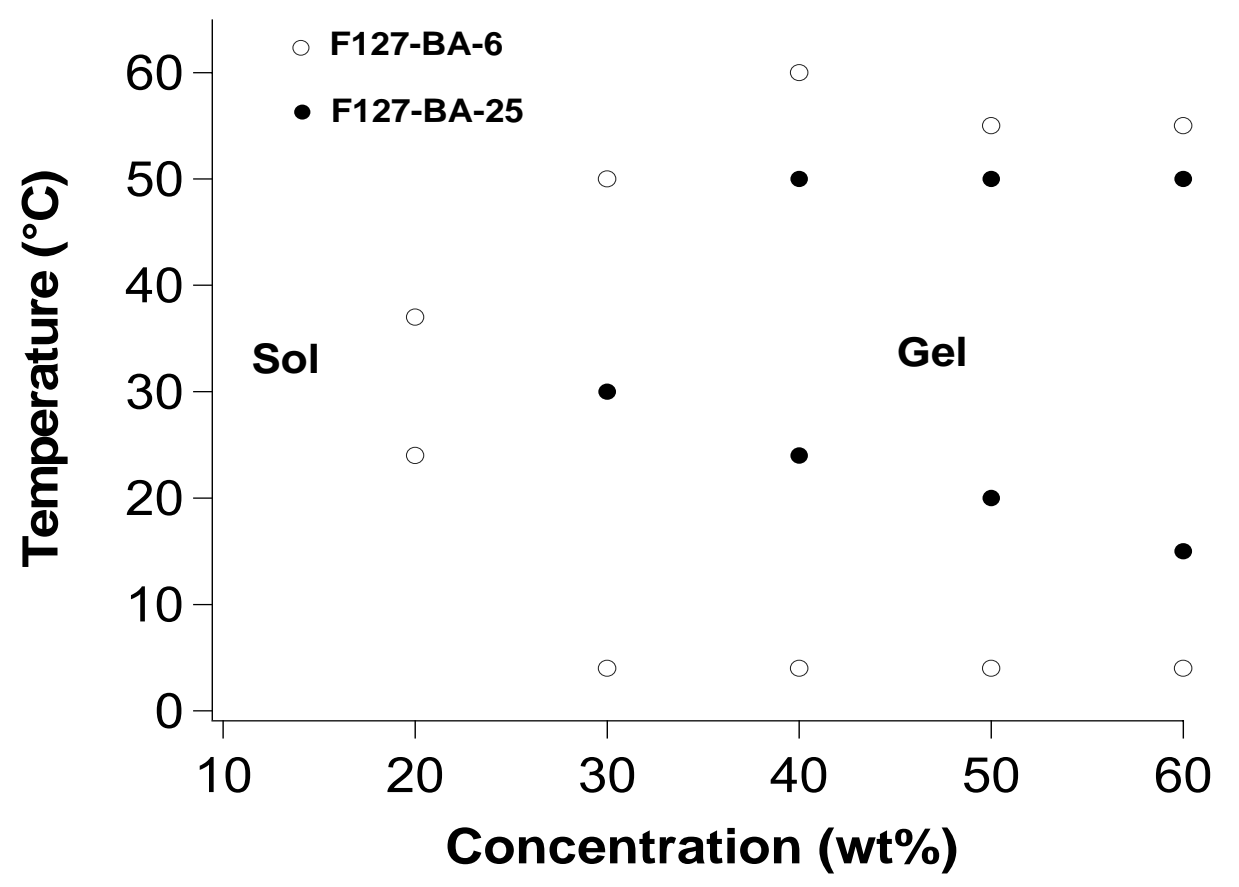

Figure. 7.4. Sol-gel phase diagram of the boronic acid pentablock copolymer. Here sol-gel refers to the solution to gelation transition phase behavior of the polymers which is determined by simple tube inversion techniques at various temperatures. The phase transition points of F127-BA-6 and F127-BA-25 polymers are shown in the figure. 
To further elucidate the solution behavior of the boronic acid pentablock copolymer, light scattering, potentiometric titration and rheological measurements were performed. The solution behavior was carried out at $\mathrm{pH} 8.5$ in aqueous media where the boronic acid is partially ionized. Light scattering measurements were performed to investigate the effect of glucose on the AABPA pentablock copolymer micellar structure. Light scattering measurements revealed that the boronic acid pentablock copolymers exhibit glucose sensitivity at $\mathrm{pH} 8.5$ aqueous solutions. In the presence of $10 \mathrm{mg} / \mathrm{ml}$ glucose, the copolymer micellar size distribution showed significant decrease in particle size for the boronic acid copolymers F127-BA-25 and F127-BA-15. When the glucose concentration was increased above $10 \mathrm{mg} / \mathrm{ml}$, the ionization reached a saturation level, and further increase in glucose concentration had no significant effect on the micellar size. In the case of F127-BA-6 polymer, there was not much effect on the micelle sizes observed. This may be due to the low content of the boronic acid blocks that were not enough to form charged complexes with glucose. Table 7.2 shows the size distribution obtained for the boronic acid pentablock copolymer with various boronic acid blocks with and without glucose.

Table 7.2. Light scattering measurements of micelle sizes ( $\mathrm{nm}$ ) of $4 \mathrm{mg} / \mathrm{ml}$ polymer solutions at $\mathrm{pH} 8.5$ with varying glucose concentrations and polymer molecular weights of two sample sets with \pm peak width deviation values from the average particle sizes

\begin{tabular}{cccc}
\hline & \multicolumn{3}{c}{ Micelle seizes $(\mathrm{nm})$} \\
\hline Glucose concentration & F127-BA-6 & F127-BA-15 & F127-BA-25 \\
\hline 0 & $39.83 \pm 5.5$ & $72.35 \pm 3.2$ & $77.34 \pm 11.06$ \\
$1 \mathrm{mg} / \mathrm{ml}$ & $38.36 \pm 5.27$ & $70.60 \pm 2.9$ & $66.87 \pm 9.5$ \\
$5 \mathrm{mg} / \mathrm{ml}$ & $33.84 \pm 4.96$ & $57.19 \pm 7.14$ & $61.59 \pm 1.37$ \\
$10 \mathrm{mg} / \mathrm{ml}$ & $31.94 \pm 4.28$ & $51.36 \pm 7.4$ & $56.84 \pm 9.45$ \\
$20 \mathrm{mg} / \mathrm{ml}$ & $37.68 \pm 5.35$ & $51.08 \pm 3.6$ & $56.17 \pm 9.17$ \\
\hline
\end{tabular}


To find out the relationship between the $\mathrm{pKa}$ value and glucose concentrations, potentiometric titration measurements were carried out. The pKa value of the boronic pentablock copolymer in water was found to be around 8.4 , while it was reduced to 8.0 and 7.7 in the presence of $5 \mathrm{mg} / \mathrm{ml}$ and $10 \mathrm{mg} / \mathrm{ml}$ glucose concentrations respectively. The complexation formation of the phenylborates with the glucose stabilizes the charged phenylborates resulting in the decrease of pKa value. Fig. 7.5 shows the titration curves of F127-BA-25 boronic acid pentablock copolymer in water with and without glucose.

Rheological measurements were performed to find the effect of glucose on the copolymer gel structure. Pentablock copolymer gels were prepared at $40 \mathrm{wt} \%$ in water at $\mathrm{pH}$ 8.5 with and without $100 \mathrm{mg} / \mathrm{ml}$ glucose concentration. The presence of glucose made the gel swell, leading to lower moduli values (Fig. 7.6). In the presence of glucose, the boronic acid pentablock copolymer formed loosely packed gels due to the stable ionization of the boronic pendant side groups. This resulted in significant loss in the moduli values of the boronic acid pentablock copolymer gels. This property can potentially be exploited to release insulin or other proteins trapped in the gels in response to glucose levels in the body.

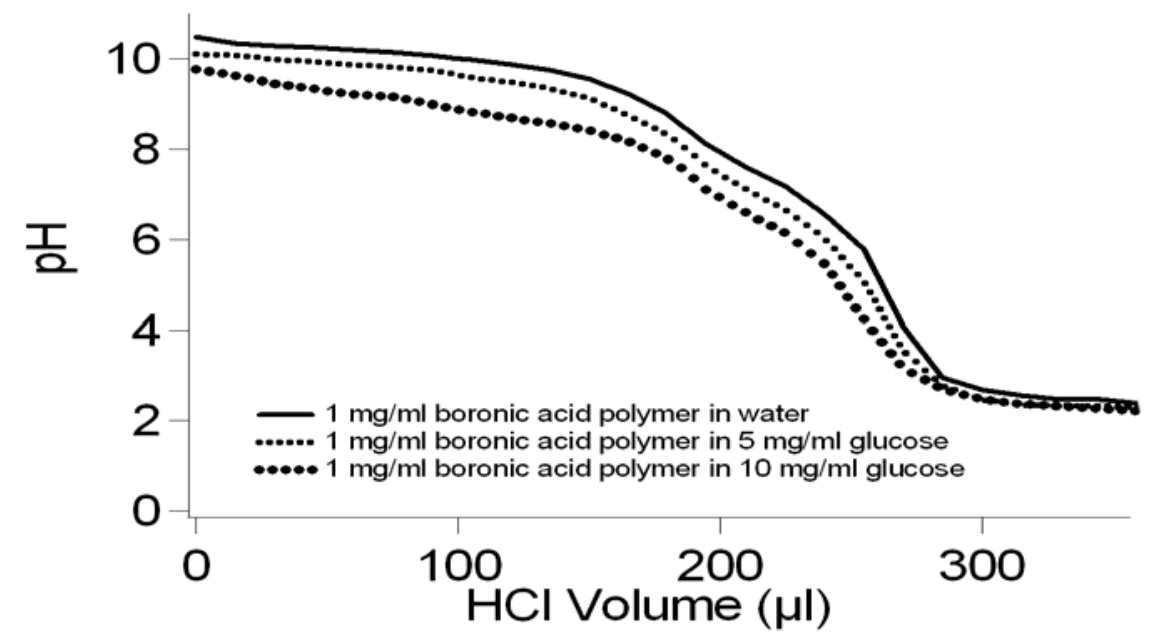

Figure.7.5. Titration curves of the boronic acid pentablock copolymer F127-BA-25 


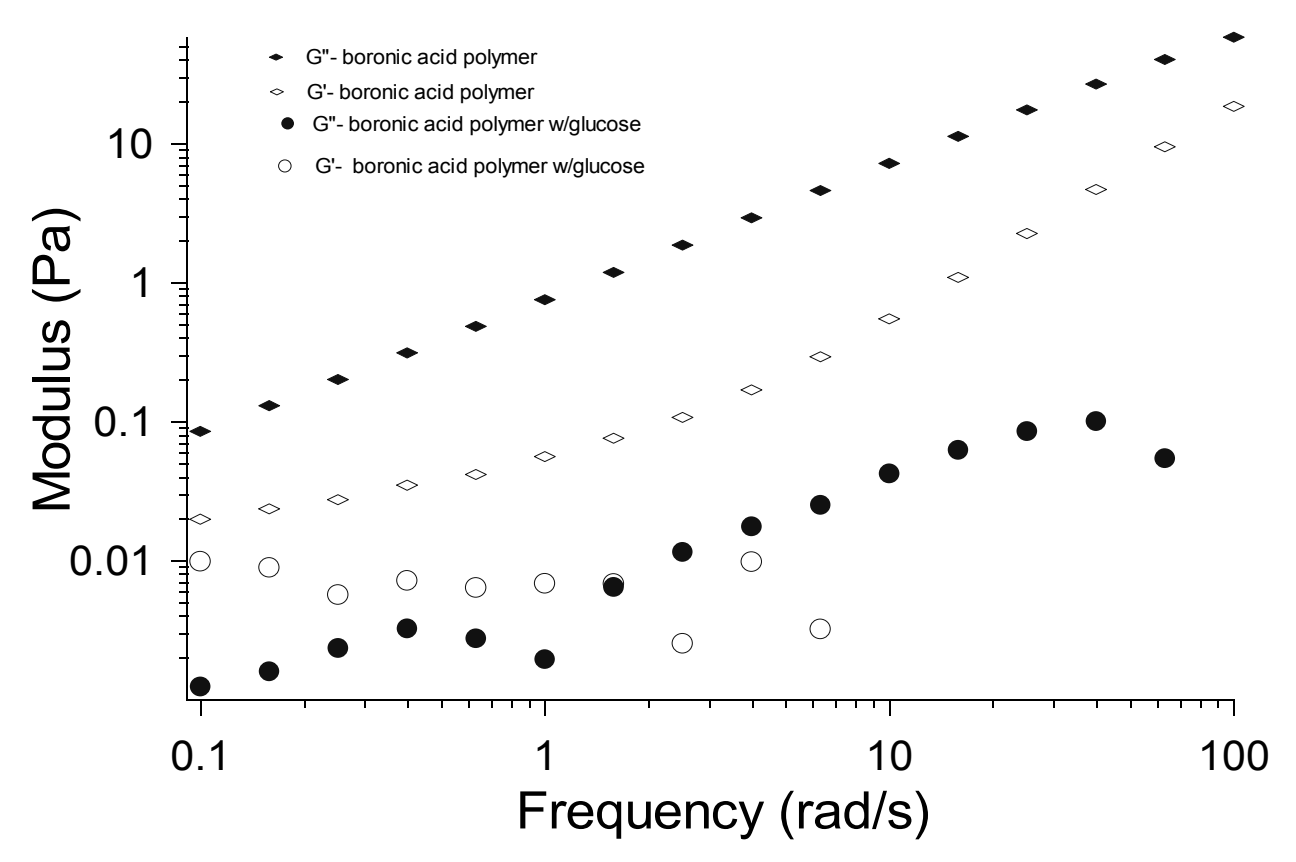

Figure.7.6. Storage and loss moduli of boronic acid- pentablock (F127-BA-25) copolymer gels $(40 \%)$ at $\mathrm{pH} 8.5$ with and without glucose $(100 \mathrm{mg} / \mathrm{ml})$ with $50 \%$ dynamic strain control.

\subsection{Conclusions}

We have demonstrated synthesis of a novel thermoresponsive glucose responsive pentablock copolymer that could potentially be used in glucose responsive applications. The novel pentablock copolymer is also capable of forming thermoreversible gels at physiological temperatures, overcoming disadvantages associated with implantation and removal of the cross-linked gels during drug delivery applications. A well-defined boronic acid pentablock copolymer was synthesized by RAFT polymerization technique. The protection of the boronic acid group with pinacol ester facilitates a well-defined RAFT polymerization of the boronic acid copolymer. The solution behavior of this copolymer was studied to find its glucose responsive properties in aqueous environments. Light scattering, potentiometric titration and rheological measurements showed that the polymer exhibits 
glucose responsive properties. Based on these initial findings that are promising, further studies will be undertaken in the future to test glucose-responsive drug delivery using these novel biomaterials.

\subsection{References}

1. B. Jeong and A. Gutowska. Trends in Biotechnology, 2002. 20(7): p. 305-311.

2. P. a. B. L. Alexandridis. Amphiphilic Block Copolymers: Self-Assembly and Applications, Elsevier, 2000: p. 1-448.

3. E. S. Gil and S. M. Hudson. Progress in Polymer Science, 2004. 29(12): p. 11731222.

4. B. G. De Geest, et al. Langmuir, 2006. 22(11): p. 5070-5074.

5. V. Lapeyre, et al. Biomacromolecules, 2006. 7(12): p. 3356-3363.

6. Y. Zhang, Y. Guan, and S. Zhou. Biomacromolecules, 2006. 7(11): p. 3196-3201.

7. T. Hoare and R. Pelton. Macromolecules, 2007. 40(3): p. 670-678.

8. H. Weith, Wiebers, J. and Gilham, P. Biochemistry, 1970. 9: p. 4396-4401.

9. A. Matsumoto, R. Yoshida, and K. Kataoka. Biomacromolecules, 2004. 5(3): p. 10381045.

10. A. Matsumoto, et al. Biomacromolecules, 2003. 4(5): p. 1410-1416.

11. C. M. Hassan, F. J. Doyle, III, and N. A. Peppas. Macromolecules, 1997. 30(20): p. 6166-6173.

12. T. Miyata, Jikihara, A., Nakamae, K., Uragami, T., Hoffman, A.S., Kinomura, K. and Okumura, M. Springer, 1996: p. pp 237-238.

13. K. Kataoka, et al. Journal of the American Chemical Society, 1998. 120(48): p. 12694-12695.

14. M. D. Determan, et al. Polymer, 2005. 46(18): p. 6933-6946.

15. M. D. Determan, J. P. Cox, and S. K. Mallapragada. Journal of Biomedical Materials Research, Part A, 2007. 81A(2): p. 326-333.

16. R. L. a. H. S. B. Letsinger. J.Am.Chem.Soc., 1959. 81: p. 3009.

17. W. J. a. S. Lennarz, H. R. J.Am.Chem.Soc., 1960. 82: p. 2169.

18. J. N. Cambre, et al. Journal of the American Chemical Society, 2007. 129(34): p. 10348-10349.

19. J. Chiefari, et al. Macromolecules, 1998. 31(16): p. 5559-5562.

20. A. B. Lowe and C. L. McCormick. Progress in Polymer Science, 2007. 32(3): p. 283351.

21. C. M. Vogels, et al. Canadian Journal of Chemistry, 1999. 77(7): p. 1196-1207.

22. T. E. Pennington, C. Kardiman, and C. A. Hutton. Tetrahedron Letters, 2004. 45(35): p. 6657-6660.

23. J. T. Lai, D. Filla, and R. Shea. Macromolecules, 2002. 35(18): p. 6754-6756.

24. A. I. Petrov, A. A. Antipov, and G. B. Sukhorukov. Macromolecules, 2003. 36(26): p. 10079-10086. 


\title{
CHAPTER 8. THERMOREVERSIBLE PHOTO CROSSLINKABLE POLYMER GELS FOR POSTTRAUMATIC ARTHRITIS CARTILAGE HEALING
}

\author{
(A paper in preparation for submission to Biomaterials) \\ Mathumai Kanapathipillai ${ }^{\mathrm{a}}$, Colin Paul ${ }^{\mathrm{b}}$, Mathew Goodman ${ }^{\mathrm{c}}$, Zhiqun Lin ${ }^{\mathrm{c}}$ and Surya K. \\ Mallapragada $^{\mathrm{a}}$
}

\subsection{Abstract}

Photocrosslinking thermoreversible gels were designed to maintain scaffold integrity for a few days to facilitate tissue regeneration and healing after trauma to minimize posttraumatic arthritis. Pluronic F127 triblock copolymers and Pluronic based pentablock copolymers with poly(diethylaminoethylmethacrylate) (PDEAEM) side blocks were modified with acrylate end groups to form injectable physically crosslinked polymer gel networks conforming to the defect sites that can be chemically crosslinked in situ via UV photopolymerization. Hydrogels were fabricated with methacrylated Pluronic/ pentablock block copolymers and polyethylene glycol diacrylate (PEG-Acr) macromers, with concentrations ranging from $10-30 \%$ to obtain strong interpenetrating crosslinked gel neworks. The chemically cross-linked gels increased the shear moduli values to about $20 \mathrm{kPa}$ compared to the physically crosslinked gel moduli values of $\sim 10 \mathrm{~Pa}$. This is further observed from solubility studies where the chemical crosslinking, resulted in swollen cross-linked polymer gels that were stable for weeks, compared to that of physical gels which dissolve within a day. Polymer macromer concentrations, UV exposure time and energy and photo initiator concentrations were optimized to obtain gels with increased crosslinking densities, and enhanced mechanical properties to withstand extreme physiologic compressive loads and support formation of new tissues. These gels could be used as injectable therapies for post- 
traumatic cartilage tissue healing due to gelation at physiological temperatures and increased moduli values that are closer to the mechanical properties of natural cartilage.

\footnotetext{
${ }^{a}$ Department of Chemical Engineering, Iowa State University and Ames Laboratory ${ }^{b}$ Department of Chemical Engineering, Arkansas State University

${ }^{c}$ Department of Materials Engineering, Iowa State University and Ames Laboratory
}

\subsection{Introduction}

Traumatic arthritis results in cartilage damage due to inability to withstand pressure or extensive load after the injury to a joint such as fracture or sprain, which eventually leads to posttraumatic osteoarthritis [1-3]. The cartilage material, if damaged and ripped away, is normally replaced by scar tissue. The scar tissue material is not as good for covering joint surfaces as the cartilage it replaces, as it is unable to support weight and is not smooth like true articular cartilage [1]. Hence under these conditions where normal physiologic reaction to fracture does not occur, surgery is a necessity, where synthetic matrices mimicking the characteristics of the cartilage environment play a role in the posttraumatic cartilage healing $[4,5]$.

Several investigations are involved in developing materials that could be used as a temporary wound healing material for posttraumatic arthritis cartilage repair [4-6]. Hydrogels seem to be a good choice as tissue engineering materials. They resemble the cell structure due to their water retaining capability from swelling, promoting cell proliferation and cell function by allowing diffusion of nutrients into the gel and cellular waste out of the gel structure $[6,7]$. The hydrogel structure has to be fine-tuned to withstand the mechanical stresses from the cellular environment as well as the degradation rates. Although several natural minerals such as alginate and fibrin hydrogels are capable of use as transplant 
materials, their mechanical properties and composition are difficult to control and difficult to mimic that of natural cartilage [8]. Synthetic hydrogels could be a better choice for the cartilage structures where the chemical structure and the degradation of the hydrogels could be easily controlled compared to the natural ones $[6,8,9]$.

Several synthetic crosslinking hydrogel networks have been used in this respect [1013]. Several methods of crosslinking hydrogels such as radical polymerization, chemical, physical, ionic and protein interactions have been currently used. Radical polymerization using UV has been the most widely used in cell applications due to its nontoxicity to cellular environments during crosslinking [7]. Vinyl monomers are used in many biomedical applications for forming cross- linking networks insitu in the presence of photoinitiator when exposed to light $[6,10,12]$. Recently several works have been focused on improving the polymer gel structure properties for cartilage tissue development. Anseth and coworkers, one of the pioneers in the development of hydrogel networks using photopolymerization of vinyl monomers, have reported increase in mechanical properties of hydrogels with good biocompatibility $[7,8]$.

Here in this work, injectable materials of Pluronic/pentablock macromers and diacryalted PEG macromer gels were fabricated with different composition ratios of macromers to optimize the gel crosslinking. The thermoreversible properties of the Pluronic allow these polymer networks be injected at the site of bone-defect cavity allowing the material to conform to the shape of the defect $[14,15]$. Without the chemical crosslinking, the physical gels themselves are not stable and dissolve in a couple of days $[12,13]$. The chemical crosslinking is designed to improve the stability of these gels and their mechanical properties. The polymer gel networks have been designed so that the degradation and moduli 
values were tuned to have the essential features needed for post traumatic bone healing. Once injected, the polymer gel can be crosslinked by UV radiation and hardened at the site of interest, which will stabilize the gels and improve its mechanical properties. These injectable crosslinking hydrogels could be versatile materials in future biomedical applications such as in posttraumatic arthritis cartilage repair.

\subsection{Materials and Methods}

Pluronic ${ }^{\circledR}$ F127, methacrylic acid,, 1,8-diazabicyclo[5.4.0]undec-7-ene (DBU) and acryloyl chloride were purchased from Sigma. Polyethylene glycol diacrylate (PEG-Acr), Mw 4000, 1000 were purchased from Polyscience Inc. Irgacure 2959 was kindly provided by Ciba-Geigy. All the other chemicals were purchased from Fisher Scientific and of high purity.

\section{Pluronic, Pentablock copolymer acrylate functunalization.}

The Pluronic initiator was synthesized by simple esterification with 2bromoisobutyryl bromide as reported previously [14]. The PDEAM pentablock copolymer was synthesized using ATRP polymerization as previously described [14]. Dimethacrylated pentablock copolymer were synthesized by a previously reported method for nucleophilic substitution of $\mathrm{Br}$ group into methacrylate functionality [16]. Briefly, the pentablock copolymer was dissolved in methylene chloride in separate flasks. Equimolar amount of methacrylic acid (MAA) to the bromine group was added to each flask under vigorous stirring after which 1,8-diazabicyclo[5.4.0]undec-7-ene (DBU) was added to each flask. The reaction was carried out for 24 hours at room temperature. The mixture was then precipitated thrice in ethyl ether and vacuum dried, and the acrylated polymers were obtained. Dimethacrylated Pluronic was synthesized by a method reported by [12]. Here the Pluronic 
was directly methacrylated by reacting with acryloyl chloride and triethylamine, 12 hours at $4^{\circ} \mathrm{C}$, and then 12 hours at room temperature as reported previously. The synthesis schemes for methacrylation of the polymers are shown below.

Scheme 1. Pentablock methacrylation

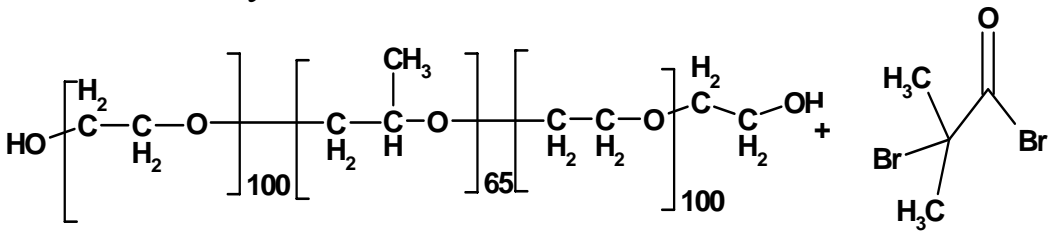

THF, triethylamine

$25^{\circ} \mathrm{C}$
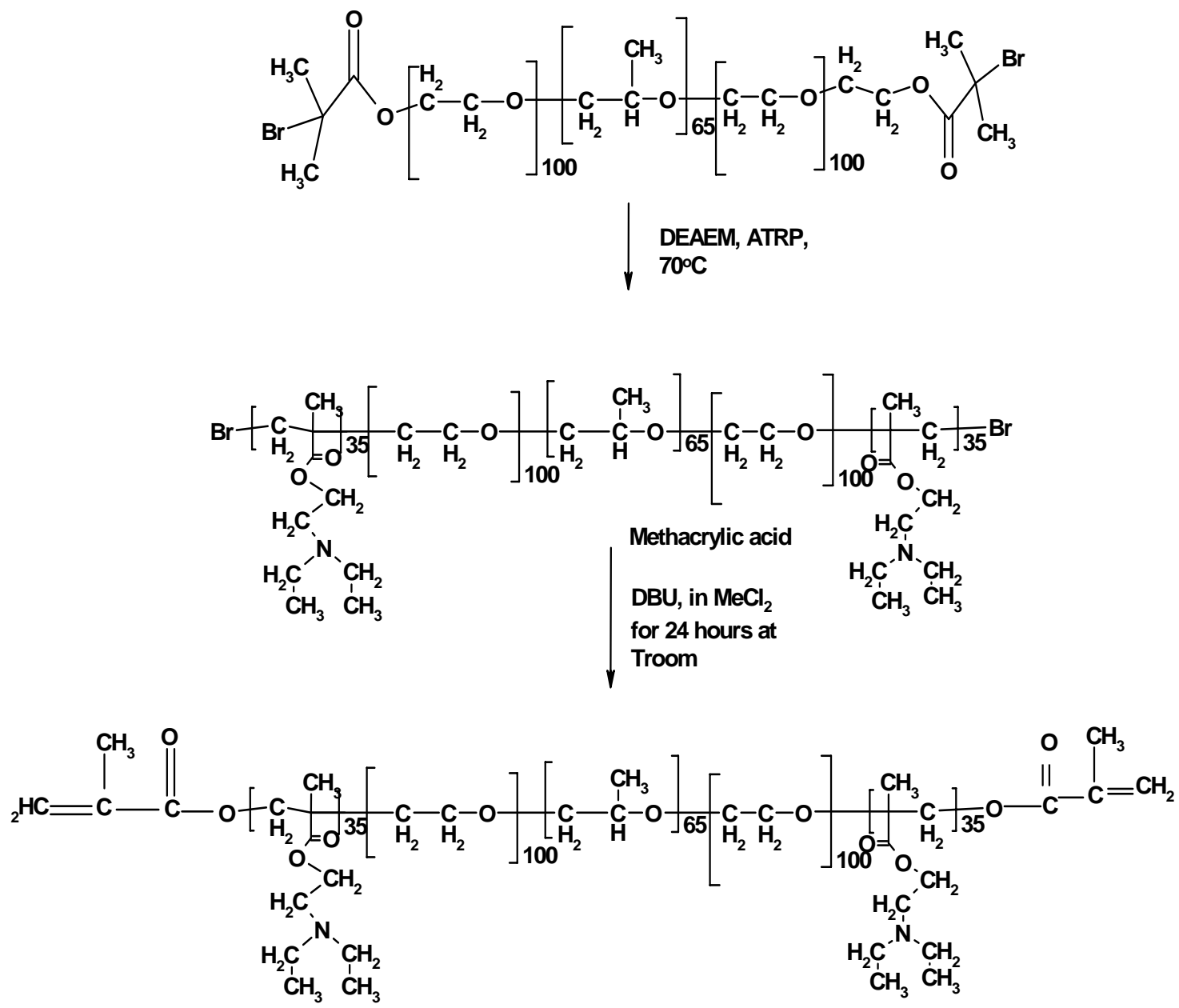
Scheme 2. Pluronic methacrylation

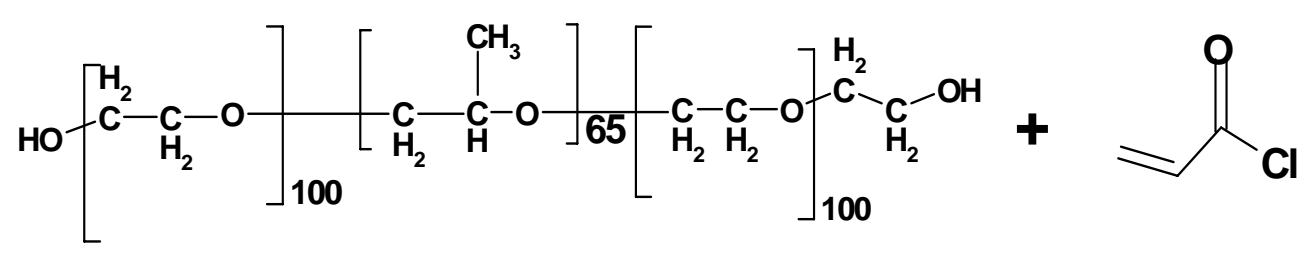

$$
\begin{aligned}
& \text { Triethylamine, } \\
& \text { in Methylene chloride } \\
& \text { at } 4 \mathrm{C}, 12 \text { hrs followed by } \\
& 12 \text { hrs at Troom }
\end{aligned}
$$

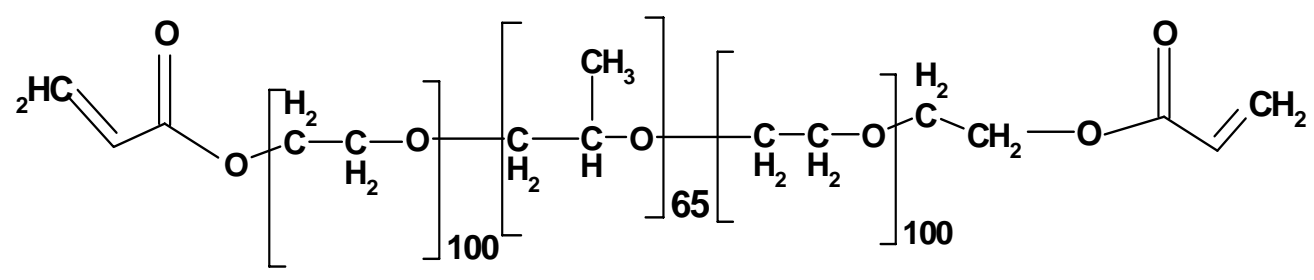

\section{Formation of crosslinking hydrogels}

Crosslinked hydrogels were formed in polypropylene wells with diameter of $16.5 \mathrm{~mm}$ and a depth of $20 \mathrm{~mm}$. Methacrylated polymer mixtures with different formulations were weighed into these wells, and $300 \mu \mathrm{L}$ of a solution of Irgacure 2959 photoinitiator in deionized water were added. The total polymer concentration in solution ranged from 20$40 \%(\mathrm{w} / \mathrm{v})$, and the concentration of the photoinitiator was ranged from $0.05 \%-0.6(\mathrm{w} / \mathrm{w})$. The solutions were dissolved at $4^{\circ} \mathrm{C}$ until a homogeneous liquid was obtained and then set at room temperature for $10-30$ min to form physical gels to mimic the state at which crosslinking would occur in physiological applications. The wells were placed beneath a UVP (Upland, CA) Blak-Ray ${ }^{\circledR}$ B-100AP UV lamp with the top of the gel $2 \mathrm{~cm}$ from the source and irradiated with $365 \mathrm{~nm}$ longwave UV light at an intensity of $21.7 \mathrm{~mW} / \mathrm{cm}^{2}$ for 10 minutes after an approximately one minute warm-up period. 


\subsection{Characterization}

Polymer molecular weights were characterized using GPC prior to crosslinking.

${ }^{1}$ HNMR spectra's were obtained with Vxr300 spectrometer. The samples were dissolved in $\mathrm{CDCl}_{3}$, and the spectra were observed to elucidate the methacrylate substitution to the polymer structures. After crosslinking, simple visual gel inversion techniques were used to characterize the morphology of the crosslinked gels. Further characterizations of the gels were performed using dissolution, swelling and mechanical studies for different mixtures of polymer macromer formulations to optimize the crosslinking conditions to obtain properties similar to that of natural cartilage.

\section{Dissolution and swelling of crosslinked gels}

Dissolution and swelling studies were performed to investigate the degree of cross linking and the degradation kinetics of the hydrogel networks. The gel samples were dissolved in water and the dissolution was monitored over time at room temperature using a simple gravimetric method as previously described [12]. Six identical samples were prepared. One sample was dried immediately after crosslinking for $20 \mathrm{~h}$ in a $37^{\circ} \mathrm{C}$ oven and weighed to determine the initial dry weight $\left(\mathrm{M}_{\mathrm{id}}\right)$ for gels of that composition. The remaining crosslinked gels were placed in $20 \mathrm{~mL}$ scintillation vials and immersed in $2 \mathrm{~mL}$ deionized water and were used for degradation and swelling studies. At selected time intervals, gels were removed from solution and were patted dry and weighed to determine swollen mass $\left(\mathrm{M}_{\mathrm{s}}\right)$. Excess water was removed from the vials containing hydrated gels, and the swollen mass was determined by subtraction of the known vial mass. The gels were then dried for $20 \mathrm{~h}$ in a $37^{\circ} \mathrm{C}$ oven and weighed to determine final dry mass $\left(\mathrm{M}_{\mathrm{fd}}\right)$. The swelling ratio of each gel was calculated using the equation, $\mathrm{Sr}=\left(\left(\mathrm{M}_{\mathrm{s}}-\mathrm{M}_{\mathrm{fd}}\right) / \mathrm{M}_{\mathrm{fd}}\right) \times 100$, where $\mathrm{M}_{\mathrm{s}}$ is 
the weight of the swollen gel and $\mathrm{M}_{\mathrm{fd}}$ is the weight of the final dried gel. The mass loss of crosslinked gels was found by using the following equation $\mathrm{M}_{\text {loss }}=\left(\mathrm{M}_{\mathrm{id}}-\mathrm{M}_{\mathrm{fd}}\right) / \mathrm{M}_{\mathrm{fd}}$. The trials were done in duplicate.

\section{Rheology and Dynamic mechanical analysis}

Rheological studies were performed to investigate the shear modulus of the crosslinked gel network. Frequency sweep tests were performed in strain controlled mode, at $37^{\circ} \mathrm{C}$ using a TA rheometer. Dynamic mechanical analysis was performed on a Perkin Elmer DMA7, to test the compressive moduli values of the cross linked gels. The test was performed at unconfined compression at a rate from $0-50 \mathrm{mN} / \mathrm{min}$ at $37^{\circ} \mathrm{C}$. The compressive moduli were calculated using the initial slope of the stress-strain curve. The hydrogels were placed in a cup and plate arrangement with dimensions of $5 \mathrm{~mm}$ in diameter and $3 \mathrm{~mm}$ in thickness. The compressive and shear moduli values were compared to similar polymer gels without crosslinking.

\subsection{Results and Discussion}

${ }^{1} \mathrm{HNMR}$ spectra were obtained to confirm the methacrylation of the polymer end groups. Figure 8.1, 8.2 shows the methacrylate signals around 5.8 -6.3 ppm, indicating the replacement of Br groups. 


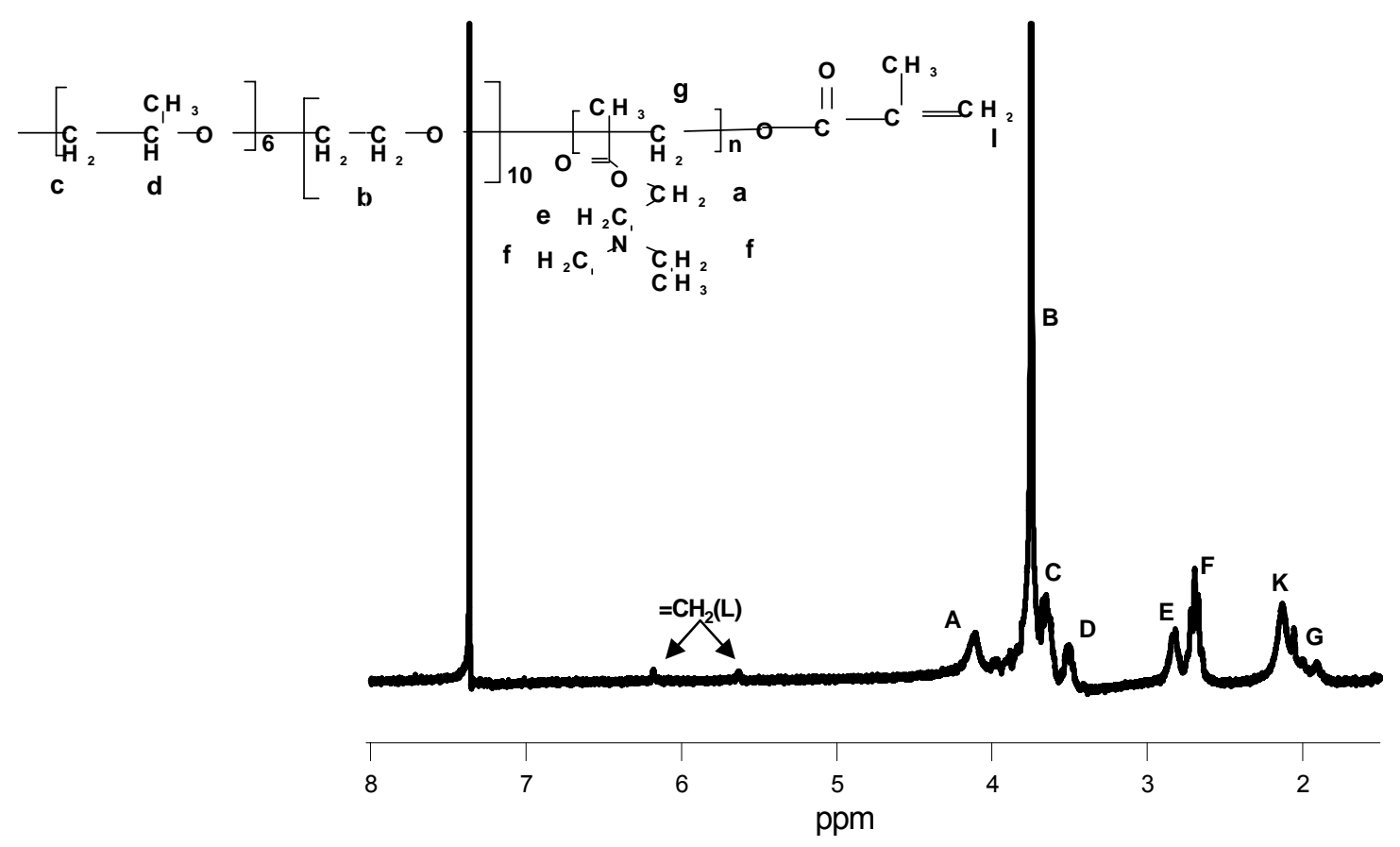

Figure 8.1. ${ }^{1} \mathrm{HNMR}$ spectra of PDEAEM pentablock methacrylation

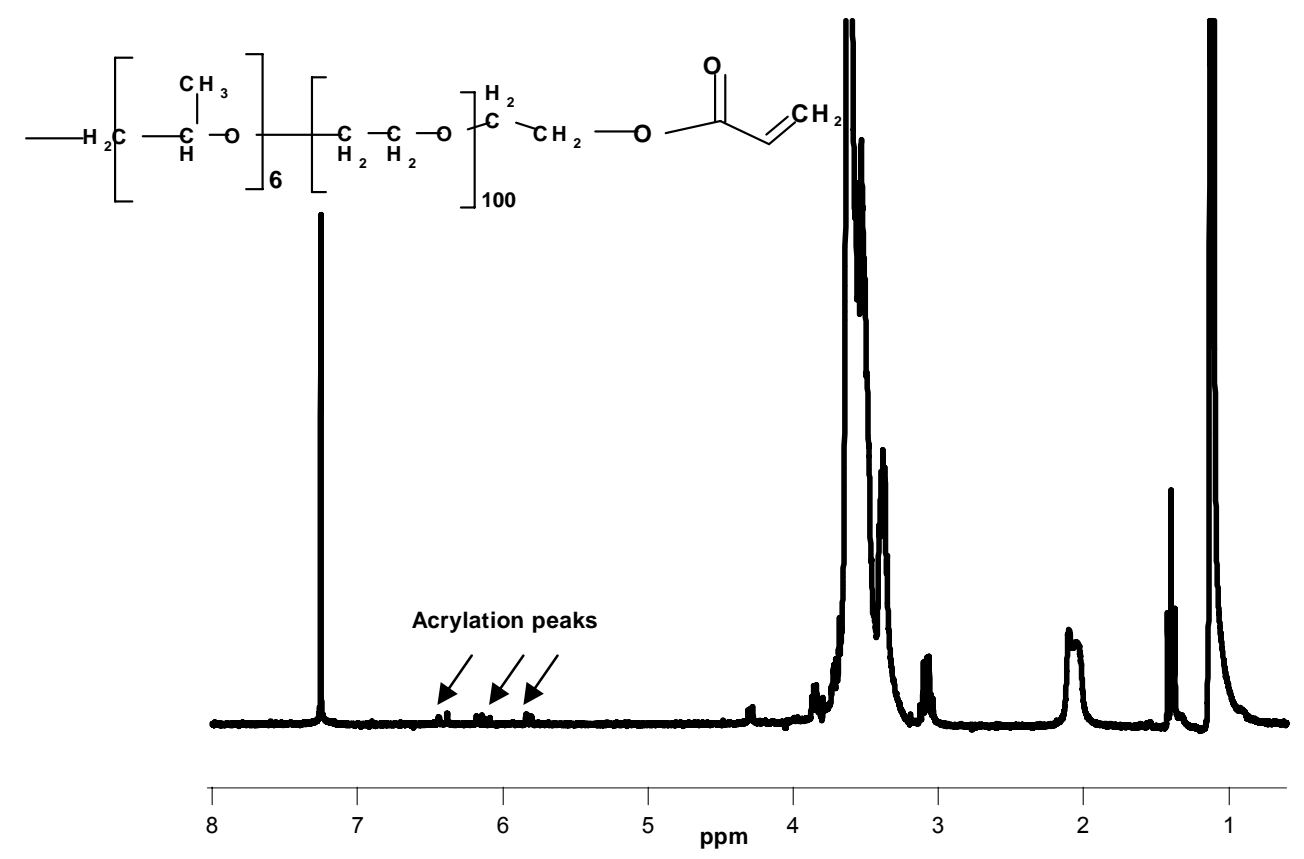

Figure 8.2. ${ }^{1} \mathrm{HNMR}$ spectra of Pluronic F127 methacrylation 
The acrylated macromers were then crosslinked under different conditions and the gels were transferred to $20 \mathrm{~mL}$ scintillation vials and soaked in $2 \mathrm{~mL}$ deionized water. The morphology of the crosslinked gels were then studied as described below. The swollen gels were classified as either lenses or hydrated gels. Lenses formed swollen, well-defined discs. Hydrated gels conformed to the shape of their container and deformed easily under applied stress. The water was replaced daily and the gels were checked for morphological changes. The timeline of fragmentation, when distinguishable, separable pieces formed, was noted. A gel was considered completely dissolved when gel fragments could no longer be distinguished from the dissolution liquid. Characteristic examples of these morphologies are shown in Figure 2.
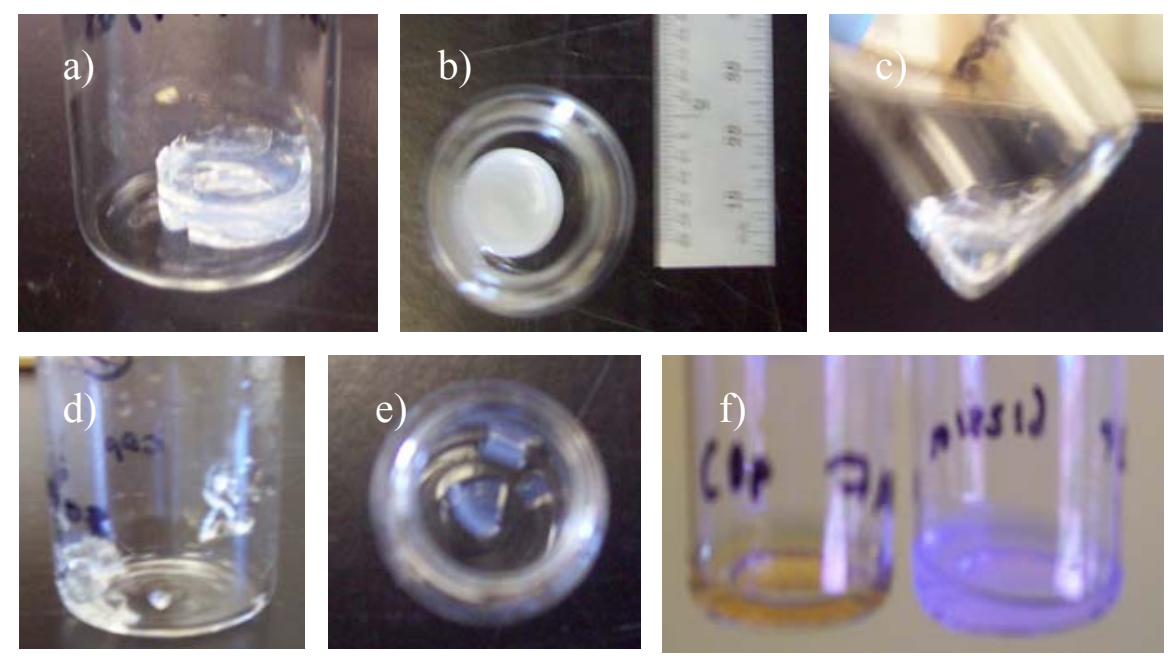

Figure 2. Characterization of crosslinked gels. After crosslinking, gels were swollen and classified as either lenses $(a, b)$ or hydrated gels $(c)$. The gels were soaked in $2 \mathrm{~mL}$ deionized water, and the date of fragmentation $(\mathrm{d}, \mathrm{e})$ and dissolution (f) was noted

The hydrogels first fragmented and then dissolved as ester linkages in the acrylate kinetic chains were hydrolytically broken. Hydrogels prepared for the dissolution study were checked daily for physical changes, and the time frame at which fragmentation and dissolution occurred was noted. Table 1 summarizes the results of these experiments. 
Table 1. Tabulated below are the gel formulations tested

\begin{tabular}{|c|c|c|c|c|c|c|c|}
\hline F127 (wt\%) & $\begin{array}{c}\text { PEG } 400 \\
\text { (wt\%) }\end{array}$ & $\begin{array}{c}\text { Initiator } \\
\text { (wt\%) }\end{array}$ & Time (min) & $\begin{array}{l}\text { Intensity } \\
\left(\mathbf{m W} / \mathbf{c m}^{2}\right)\end{array}$ & Morphology & Fragment (days) & $\begin{array}{l}\text { Dissolution } \\
\text { (days) }\end{array}$ \\
\hline 25 & 5 & 0.3 & 30 & 21.7 & Hydrated gel & 3 & 9 \\
\hline 20 & 10 & 0.3 & 30 & 21.7 & Cracked lens & 2 & 3 \\
\hline F127 (wt\%) & $\begin{array}{c}\text { PEG } \\
1000 \\
(w t \%)\end{array}$ & $\begin{array}{c}\text { Initiator } \\
\text { (wt\%) }\end{array}$ & Time (min) & $\begin{array}{l}\text { Intensity } \\
\left(\mathrm{mW} / \mathrm{cm}^{2}\right)\end{array}$ & Morphology & Fragment (days) & $\begin{array}{l}\text { Dissolution } \\
\text { (days) }\end{array}$ \\
\hline 25 & 5 & 0.05 & 10 & 21.7 & Hydrated gel & $1-2$ & $1-2$ \\
\hline 30 & 10 & 0.05 & 10 & 21.7 & Hydrated gel & $1-2$ & $3-5$ \\
\hline 15 & 15 & 0.05 & 10 & 21.7 & Cloudy lens & $>11$ & $>11$ \\
\hline 20 & 20 & 0.05 & 10 & 21.7 & Cloudy lens & $>11$ & $>11$ \\
\hline 15 & 20 & 0.05 & 10 & 21.7 & Cloudy lens & $>8$ & $>8$ \\
\hline F127 (wt\%) & $\begin{array}{c}\text { PEG } \\
4000 \\
(w t \%) \\
\end{array}$ & $\begin{array}{c}\text { Initiator } \\
\text { (wt\%) }\end{array}$ & Time (min) & $\begin{array}{c}\text { Intensity } \\
\left(\mathbf{m W} / \mathbf{c m}^{2}\right)\end{array}$ & Morphology & Fragment (days) & $\begin{array}{l}\text { Dissolution } \\
\text { (days) }\end{array}$ \\
\hline 25 & 5 & 0.05 & 30 & 21.7 & Hydrated gel & $>11$ & $>11$ \\
\hline 25 & 5 & 0.3 & 30 & 21.7 & Hydrated gel & 24 & 33 \\
\hline 30 & 10 & 0.046 & 30 & 21.7 & Lens & 2 & $>29$ \\
\hline 20 & 10 & 0.3 & 30 & 21.7 & Hydrated gel & 8 & $>29$ \\
\hline 15 & 15 & 0.05 & 30 & 21.7 & Lens & $>11$ & $>11$ \\
\hline 15 & 15 & 0.3 & 30 & 21.7 & Lens & 30 & $>32$ \\
\hline 20 & 20 & 0.046 & 30 & 21.7 & Lens & 13 & $>29$ \\
\hline 15 & 20 & 0.05 & 30 & 21.7 & Lens & $>23$ & $>23$ \\
\hline $\begin{array}{c}(\text { PDEAEM) } \\
\text {-Mac(wt\%) }\end{array}$ & $\begin{array}{c}\text { PEG } \\
1000 \\
(w t \%)\end{array}$ & $\begin{array}{c}\text { Initiator } \\
\text { (wt\%) }\end{array}$ & Time (min) & $\begin{array}{c}\text { Intensity } \\
\left(\mathrm{mW} / \mathrm{cm}^{2}\right)\end{array}$ & Morphology & Fragment (days) & $\begin{array}{l}\text { Dissolution } \\
\text { (days) }\end{array}$ \\
\hline 15 & 15 & 0.05 & 30 & 21.7 & Gel/lens mix & 2 & $>8$ \\
\hline 15 & 25 & 0.05 & 30 & 21.7 & Gel/lens mix & 2 & $>8$ \\
\hline $\begin{array}{c}(\text { PDEAEM) } \\
(\mathrm{wt} \%)\end{array}$ & $\begin{array}{c}\text { PEG } \\
1000 \\
(w t \%)\end{array}$ & $\begin{array}{c}\text { Initiator } \\
\text { (wt\%) }\end{array}$ & Time (min) & $\begin{array}{c}\text { Intensity } \\
\left(\mathrm{mW} / \mathrm{cm}^{2}\right)\end{array}$ & Morphology & Fragment (days) & $\begin{array}{l}\text { Dissolution } \\
\text { (days) }\end{array}$ \\
\hline 15 & 15 & 0.05 & 30 & 21.7 & Gel/lens mix & $1-2$ & $>8$ \\
\hline $\begin{array}{c}\text { (PDEAEM) } \\
(\mathrm{wt} \%)\end{array}$ & $\begin{array}{c}\text { PEG } \\
4000 \\
(w t \%)\end{array}$ & $\begin{array}{l}\text { Initiator } \\
\text { (wt\%) }\end{array}$ & Time (min) & $\begin{array}{c}\text { Intensity } \\
\left(\mathrm{mW} / \mathrm{cm}^{2}\right)\end{array}$ & Morphology & Fragment (days) & $\begin{array}{l}\text { Dissolution } \\
\text { (days) }\end{array}$ \\
\hline 30 & 10 & 0.046 & 30 & 21.7 & Hydrated gel & 1 & 2 \\
\hline 20 & 20 & 0.046 & 30 & 21.7 & Lens & 1 & $>29$ \\
\hline 15 & 15 & 0.3 & 30 & 21,7 & Hydrated gel & 10 & $>32$ \\
\hline 20 & 20 & 0.05 & 10 & 21,7 & Hydrated gel & $1-2$ & $1-2$ \\
\hline F127 (wt\%) & $\begin{array}{c}\text { (PDEAE } \\
\text { M) } \\
(w 5 \%)\end{array}$ & $\begin{array}{l}\text { Initiator } \\
\text { (wt\%) }\end{array}$ & Time (min) & $\begin{array}{c}\text { Intensity } \\
\left(\mathbf{m W} / \mathbf{c m}^{2}\right)\end{array}$ & Morphology & Fragment (days) & $\begin{array}{l}\text { Dissolution } \\
\text { (days) }\end{array}$ \\
\hline 30 & 0 & 0.05 & 10 & 21.7 & lens & $>10$ & $>10$ \\
\hline 0 & 40 & 0.046 & 30 & 21.7 & Physical gel & N/A & 2 \\
\hline 15 & 15 & 0.046 & 30 & 21.7 & N/A & $\mathrm{N} / \mathrm{A}$ & 1 \\
\hline 20 & 20 & 0.046 & 600 & 21.7 & Physical gel & N/A & $2-5$ \\
\hline
\end{tabular}


Note. Here the polymers tabulated are all acrylated macromers. As a control, F127 and PDEAEM pentablock copolymers without acrylation were tested for gel dissolution, and the gels were observed to dissolve fully within 12 hours.

Hydrogels with a higher mass percentage of PEG-derived acrylates took longer to fragment and dissolved more slowly than those with a smaller amount of PEG. This effect is explained by consideration of the crosslinking density in the hydrogel. Because PEG has a lower molecular weight than F127 or pentablock copolymer, the initial double bond concentration in the photopolymerizable solution is higher when a larger percentage of PEG is incorporated. Therefore, the denser crosslinking sites, help to form tighter interpenetrating networks, resulting in slower dissolution rate due to the difficulty for water to penetrate the network and to cleave the ester bonds hydrolytically. Control samples containing methacrylated F127 or pentablock, shown in the final section of Table 1, did not form stable crosslinked hydrogels under identical conditions, corroborating the importance of initial double bond density. In general, hydrogels formed with nearly equal F127: PEG mass ratios formed more stable gels. Based on these studies, the sample conditions that took longer to dissolve were chosen for the dissolution, swelling and mechanical studies, and the results were tabulated below.

Table 2. Swelling, dissolution and mechanical studies

\begin{tabular}{cccccccc}
\hline F127 (wt\%) & $\begin{array}{c}\text { PEG 1000 } \\
\text { (wt \%) }\end{array}$ & $\begin{array}{c}\text { PEG4000 } \\
\text { (wt \%) }\end{array}$ & $\begin{array}{c}\text { \% Mass loss } \\
\text { (10 days) }\end{array}$ & $\begin{array}{c}\text { \% Swelling (10 } \\
\text { days) }\end{array}$ & $\begin{array}{c}\text { Compresssive } \\
\text { moduls (KPa) }\end{array}$ & $\begin{array}{c}\text { Shear } \\
\text { Modulus } \\
\text { (KPa) }\end{array}$ \\
\hline 20 & 20 & 0 & 46.4 & 5.58 & $\sim 50$ & $18-22$ \\
15 & 15 & 0 & 49.3 & 6.46 & $\sim 50$ & $18-22$ \\
20 & 0 & 10 & 54.5 & 19.36 & $\sim 25$ & $10-12$ \\
15 & 0 & 15 & 47.1 & 13.69 & $\sim 30$ & $18-22$ \\
30 & 0 & 0 & N/A & N/A & $\sim 10$ & N/A
\end{tabular}


Swelling data provides additional insights into the relationship between gel composition and crosslinking density. Hydrogels with F127 to PEG 1000 ratios of 1:1 showed swelling ratios of 5-7 over the course of the study. The values remained nearly constant and were noticeably less than those of gels containing PEG 4000. This supports the theory that a higher initial double bond concentration produces a more densely crosslinked network. The swelling ratios are similar to those reported for hydrogels formed from crosslinking F127 with hyaluronic acid [17] or chitosan [12]. Transparent hydrogels with F127 to PEG 4000 ratios of 1:1 showed similar stasis in swelling ratio, which remained between 14-20, suggesting that these gels were also stably crosslinked.

For mass loss data, initially in all gel compositions, unreacted polymer washed out of the hydrogel during the first two days of dissolution, resulting in a mass loss of $\sim 33 \%$. The mass loss of hydrogels observed with Pluronic:PEG ratio of less than or equal to 1:1 was nearly constant after the first few days. From two replicates, the percentage mass loss for these gels over 8-10 days was observed to be about 50\%.

Hydrogels formed from pentablock copolymer appear less promising for cartilage engineering applications. The hydrogels fragmented quickly, typically in 1-2 days. This may be due to the fact that the pentablock copolymers form physical gels at lower temperature than Pluronic for a given mass concentration, making even distribution of polymer and initiator more difficult and leading to the lack of compositional homogeneity in the final crosslinked hydrogel. Further, the higher MW of the pentablock copolymers may have made them less mobile in solution during the crosslinking reaction.

We have successfully, designed thermoreversible injectable insitu cross-linking gel networks with improved mechanical properties and stability. As far as the moduli values are 
concerned, the gel mechanical properties still need to be improved about 10 fold to reach the natural cartilage compressive moduli values which are in the range of 500-600 $\mathrm{kPa}$. Future directions of this work will be to increase the gel moduli with biomineralization strategies by forming a nanocomposite calcium phosphate-polymer crosslinked gel network. For further integration of these gels into the cartilage environment, these hydrogel networks will be conjugated with collagen binding peptides. This will help to mimic the natural cartilage environment and would increase the mechanical properties of the gel due to the inorganic content. These networks could be used as injectable cartilage matrices for applications such as post traumatic arthritis healing.

\subsection{Acknowledgements}

This work was supported by the University of Iowa.

\subsection{References}

1. Repo, R.U. and J.B. Finlay, Survival of articular cartilage after controlled impact. J Bone Joint Surg Am FIELD Full Journal Title:The Journal of bone and joint surgery. American volume, 1977. 59(8): p. 1068-76.

2. Borrelli, J., Jr., et al., Effect of impact load on articular cartilage: development of an intra-articular fracture model. J Orthop Trauma FIELD Full Journal Title:Journal of orthopaedic trauma, 1997. 11(5): p. 319-26.

3. Chen, C.-T., et al., Time, stress, and location dependent chondrocyte death and collagen damage in cyclically loaded articular cartilage. Journal of Orthopaedic Research, 2003. 21(5): p. 888-898.

4. $\quad$ Nesic, D., et al., Cartilage tissue engineering for degenerative joint disease. Advanced Drug Delivery Reviews, 2006. 58(2): p. 300-322.

5. Khan, Y., et al., Tissue engineering of bone: material and matrix considerations. J Bone Joint Surg Am FIELD Full Journal Title:The Journal of bone and joint surgery. American volume, 2008. 90 Suppl 1: p. 36-42.

6. $\quad$ Martens, P.J., S.J. Bryant, and K.S. Anseth, Tailoring the Degradation of Hydrogels Formed from Multivinyl Poly(ethylene glycol) and Poly(vinyl alcohol) Macromers for Cartilage Tissue Engineering. Biomacromolecules, 2003. 4(2): p. 283-292.

7. Bryant, S.J., C.R. Nuttelman, and K.S. Anseth, Cytocompatibility of UV and visible light photoinitiating systems on cultured NIH/3T3 fibroblasts in vitro. Journal of Biomaterials Science, Polymer Edition, 2000. 11(5): p. 439-457. 
8. Bryant, S.J., et al., Encapsulating chondrocytes in degrading PEG hydrogels with high modulus: Engineering gel structural changes to facilitate cartilaginous tissue production. Biotechnology and Bioengineering, 2004. 86(7): p. 747-755.

9. Martens, P. and K.S. Anseth, Characterization of hydrogels formed from acrylate modified poly(vinyl alcohol) macromers. Polymer, 2000. 41(21): p. 7715-7722.

10. Sawhney, S.A., Pathak, C. P., Hubbell, J. A., Biodegradable hydrogels based on photopolymerized poly(ethylene-glycol)-co-poly(alpha-hydroxy acid) diacrylate macromers. Macromolecules, 1993. 26: p. 581-587.

11. Huang, X., et al., Porous Thermoresponsive-co-Biodegradable Hydrogels as TissueEngineering Scaffolds for 3-Dimensional In Vitro Culture of Chondrocytes. Tissue Engineering, 2007. 13(11): p. 2645-2652.

12. Yoo, H.S., Photo-cross-linkable and thermo-responsive hydrogels containing chitosan and Pluronic for sustained release of human growth hormone (hGH). Journal of Biomaterials Science, Polymer Edition, 2007. 18(11): p. 1429-1441.

13. Yoon, J.J., H.J. Chung, and T.G. Park, Photo-crosslinkable and biodegradable Pluronic/heparin hydrogels for local and sustained delivery of angiogenic growth factor. Journal of Biomedical Materials Research, Part A, 2007. 83A(3): p. 597-605.

14. Determan, M.D., et al., Synthesis and characterization of temperature and $\mathrm{pH}$ responsive pentablock copolymers. Polymer, 2005. 46(18): p. 6933-6946.

15. Determan, M.D., J.P. Cox, and S.K. Mallapragada, Drug release from $\mathrm{pH}$-responsive thermogelling pentablock copolymers. Journal of Biomedical Materials Research, Part A, 2007. 81A(2): p. 326-333.

16. Yang, W., et al., Bulk synthesis of poly(tert-butyl methacrylate) long macromonomer with narrow distribution by atom transfer radical polymerization and nucleophilic substitution. Chemistry Letters, 2006. 35(2): p. 222-223.

17. Chun, K.W., et al., Controlled release of plasmid DNA from photo-cross-linked pluronic hydrogels. Biomaterials, 2005. 26(16): p. 3319-3326. 


\section{CHAPTER 9. GENERAL CONCLUSIONS AND FUTURE DIRECTIONS}

\subsection{General Conclusions}

We have developed a family of block copolymers with $\mathrm{pH}$, temperature, and glucose responsive properties, and block copolymer-peptide conjugates with bio specificity to enhance templated nucleation and growth of inorganic minerals. These block copolymers can self-assemble and form hierarchical structures over multiple length scales and are potential candidates for biomineralization and biomedical applications. The polymer-templated directed synthesis of inorganic minerals completely by self-assembly, with hierarchical structural order on the nanometer scale is a novel contribution of this work. Chapters 4-6 of this thesis detail the work on the role of these block copolymers on biomimetic mineral synthesis. Although we have successfully designed ionic templates for materials synthesis from a bottom-up approach, the yield obtained for the inorganic precipitation as well as the mechanical properties still need to be improved compared to that seen in nature. One of the reasons is the high percentage of polymer template needed for the subsequent self-assembled polymer-inorganic gel formation. To overcome this limitation, future directions of this study will be focused on organic polypeptide hydrogel templates that form gels at low concentrations. Further, in a parallel approach, we are also developing protein-polymer hydrogel templates for site-specific and controlled architecture synthesis of biominerals in future. The bottom-up approach of the synthesis process described in this work, completely by self-assembly of organic-inorganic precursors, yet need to be completely understood fully at a molecular level. Future collaborative work with Dr. P. Thiyagarajan involving the detailed analysis of the data obtained from the complementary small angle neutron and X-ray scattering for these block copolymers and their corresponding nanocomposite micellar self- 
assembly in dilute aqueous solutions, will provide further insights into the self-assembly, nucleation and the subsequent growth of these mineral nanocomposites.

\subsection{Future directions}

\subsubsection{Detailed analysis and modeling of small angle scattering data of temperature dependent phase behavior of ionic pentablock copolymers and polymer nanocomposites micelles in dilute aqueous solutions (Collaboration with Argonne National Lab)}

Aqueous solutions of the ionic pentablock copolymers were observed to form micelles and gels in response to changes in temperature and $\mathrm{pH}$ due to the lower critical solution temperature of the PPO block and the polyelectrolyte character of the PAA and polysufobetaine blocks respectively. The zwitterionic pentablock copolymers show interesting phase behavior due to the LCST and UCST behavior of different blocks of the copolymer. The temperature dependent phase behaviors of the polymer and the nanocomposite micelles were studied using small angle scattering, NMR and rheological measurements. The scattering data for the nanocomposite micelles formed by the templating of these polymers show no thermo responsive behavior suggesting that the micelles are stable in the presence of inorganic. Complementary small angle neutron and X-ray scattering for these block copolymers and their corresponding nanocomposite micellar self-assembly in dilute aqueous solutions will help to elucidate the roles of organic and inorganic phases in the self-assembly process. Modeling of the small angle scattering data will allow for quantitative assessment of micellar dimensions and shapes and will provide insights on the nucleation and the growth of the inorganic on the polymer micelles. Recently an efficient model was

obtained by Determan et al. for cationic pentablock copolymer micelle systems [1]. Due to the polyelectrolyte present in the pentablock system, the micelles were modeled as a compact 
hydrophobic core surrounded by double corona layers. A form factor of concentric spherical shell of 3 regions and the Hayter-Penfold macroion potential structure factor that accounts for the electrostatic interactions between charged colloidal particles, when used together, yielded better fits to the scattering data obtained for these micelles [1]. Similar models will be a good start for our pentablock system, which also consists of polylelectrolyte side blocks, although in the case of the nanocomposite micelles, the system may have to be considered differently due to the presence of the inorganic layer around the micelle corona. Initial approaches to model our system will be based on the modeling studies done by Determan et al [1].

\subsubsection{Design of artificial Polypeptide scaffolds}

\subsubsection{Organic polypeptides as templates for biomineralization}

It was found that unnatural amphiphilic polypeptides are capable of forming self assembled gels from the hydrophobic $\alpha$-helical domain of the peptides [2-4]. Hydrogel formation was discovered by Deming and co-workers $[2,5,6]$ with a series of diblock copolypeptides containing water solubilizing charged domains of either poly(L-lysine) or poly(L-glutamic acid) and with an $\alpha$-helical hydrophobic domain of poly(L-leucine), which were named as $\mathrm{K}_{m} \mathrm{~L}_{\mathrm{n}}$ or $\mathrm{E}_{\mathrm{m}} \mathrm{L}_{\mathrm{n}}$ polypeptides, where $\mathrm{m}$ and $\mathrm{n}$ represent the number of blocks of each aminoacid [2]. The characterization study revealed that the hydrogel assembly was taking place through the association of $\alpha$-helices perpendicular to the long fibril axis dimension $[2,7]$. Further they are capable of forming gels at concentrations as low as 0.1 $\mathrm{wt} \%$ in water with moduli values in the range of $10-500 \mathrm{~Pa}[5]$. The gel formation seems to be affected in the presence of increased ionic strength, due to the disruption of interchain repulsions of the hydrophilic blocks of the peptides, although it may be overcome by the 
chain coiling due to the charge screening of the hydrophilic domain, which will introduce steric repulsions which will help to maintain the hydrogel structure. The polypeptides were synthesized by N-carboxyanhydride (NCA) polymerizations developed by Deming and coworkers [3].

These polypeptide hydrogels provide more efficient templating in biomaterials synthesis due to their ability to self-assemble and form gels at concentrations as low as $0.25 \%[2,5]$. Hence it will facilitate a higher fraction of inorganic packing, which will enhance the mechanical properties of the designed material. By synthesizing polypeptides mimicking the natural protein templates, we can not only attain a polymer template with complex nucleation sites for inorganic interactions, but also accommodate a high inorganic content due to the smaller amount of protein polymer needed for gelation.

In collaboration with Dr. Deming's lab at UCLA, we have synthesized $\mathrm{K}_{170} \mathrm{~L}_{30}$ block copolypeptide and are currently conducting experiments with these polypeptide gels for our mineral synthesis. Our initial studies show higher inorganic content with these polypeptide gels with hierarchical structural order of the nanocomposite gels. Figure 9.1 shows the TGA data obtained for these nanocomposite gels, in which an inorganic content of $56 \%$ is observed, which is higher than the inorganic fractions obtained with other synthetic block copolymer templates. Further the XRD data (Figure 9.2) shows that the inorganic phase is indeed hydroxyapaptite. These results indicate promising templating abilities of these polypeptide gels for future biomineralization studies. 


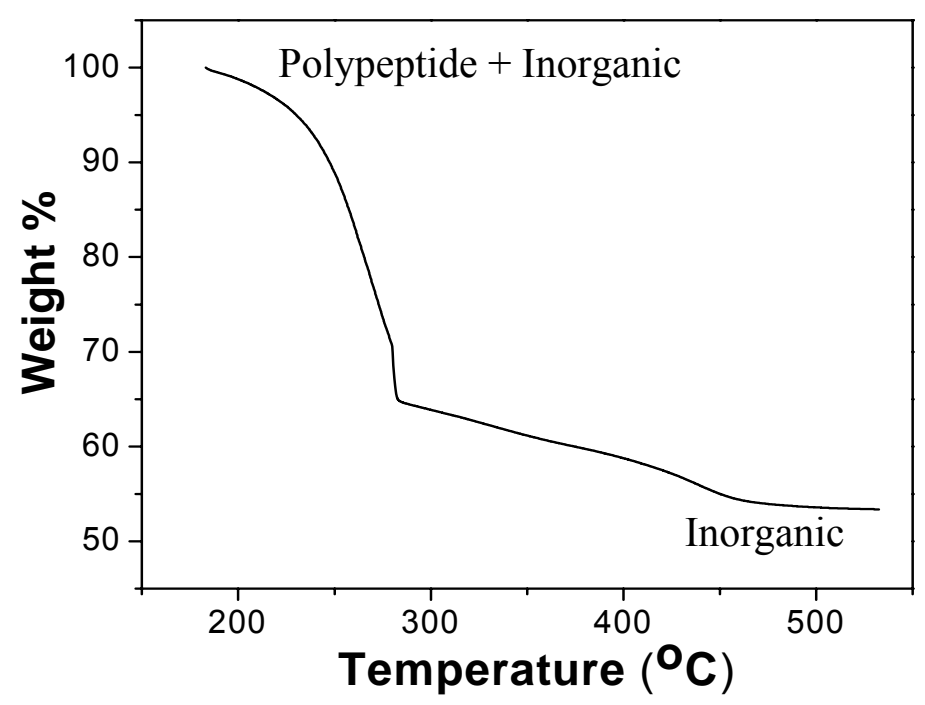

Figure 9.1. Thermogravimetric analysis of polymer inorganic gel nanocomposite (Samples were dried before the analysis to remove the water) (Data obtained by Yusufoglu)

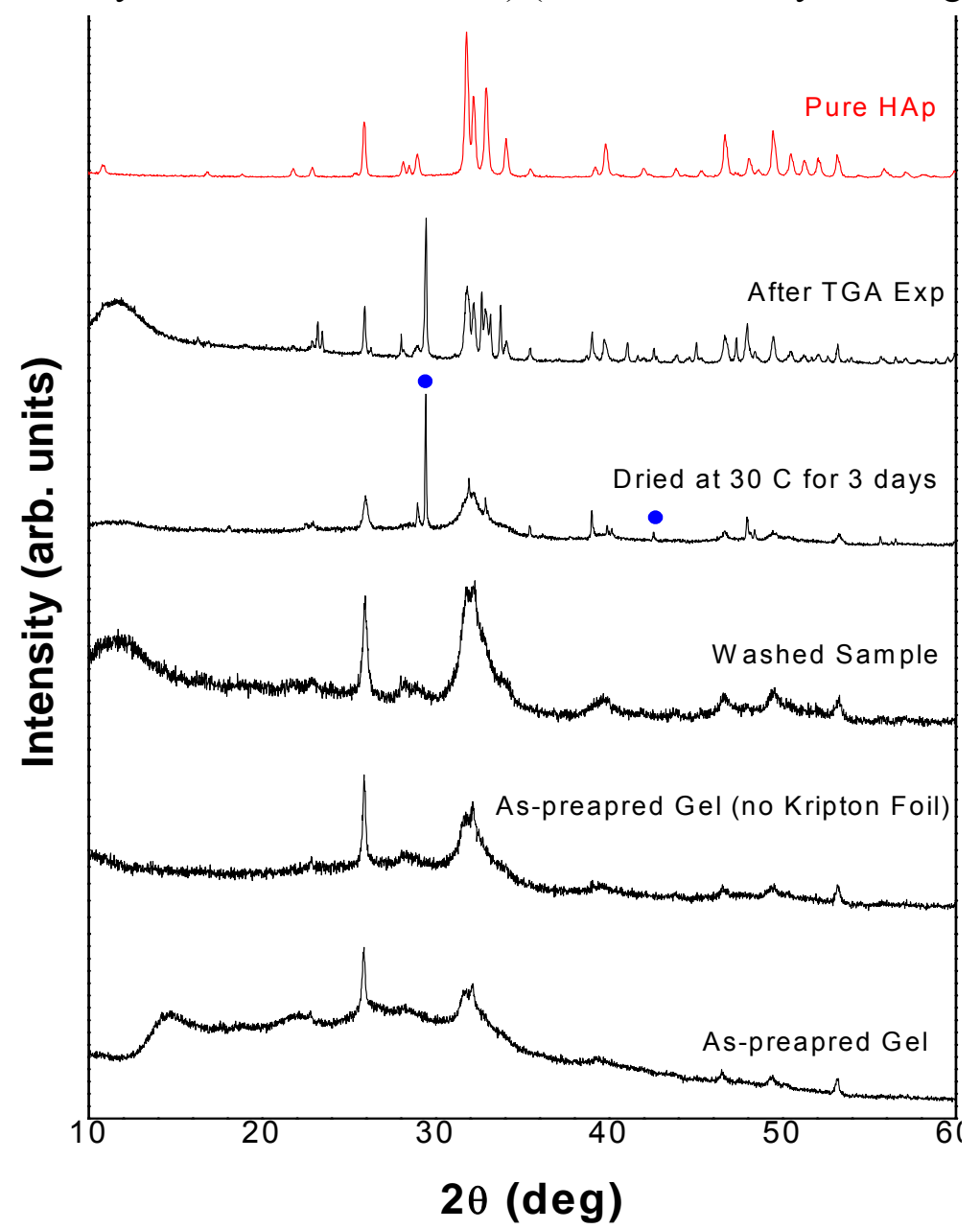

Figure 9.2. XRD of the polypeptide gel nanocomposites at different drying conditions (Data obtained by Yusufoglu) 


\subsubsection{Biopolypeptide scaffolds}

\section{Artificial polypeptide scaffold design for Mms6 protein immobilization}

In addition to calcium phosphate mineralization seen in Nature, other examples of biomineralization include production of magnetic nanocrystals by magnetotactic bacteria. Mms6 protein is thought to be involved in the mineralization of magnetic crystals in bacterial magnetosomes [8]. Magnetic crystals have vast range of applications from ultra high density storage to magnetic resonance imaging in cancer detection. Hence the synthesis of magnetic crystals, with site-specific Mms6 protein templates has lots of potential in biomimetic research. Site-specific Mms6 protein network will provide a better control of the size and shape for the synthesis of magnetic nanocrystals using a biomimetic approach. With the collaboration of Dr. David Tirrell's group at Caltech we are developing an artificial polypeptide scaffold that could be used to synthesize nanocrystals with controlled architecture and specificity.

The goal is to develop an artificial polypeptide scaffold for the immobilization of Mms6 protein to synthesize an array of nanoparticles with size and shape specificity. The polypeptide design consists of separate surface anchor and protein capture domains and uses an artificial aminoacid for surface cross-linking. The design approach is shown in Figure 9.3. The protein capture domain functions through the coiled-coil association of a leucine zipper pair [9-11] designated as ZE and ZR. Here an elastin mimetic domain with a paraazidophenylalanine group is used for the surface anchorage as previously described [9]. 

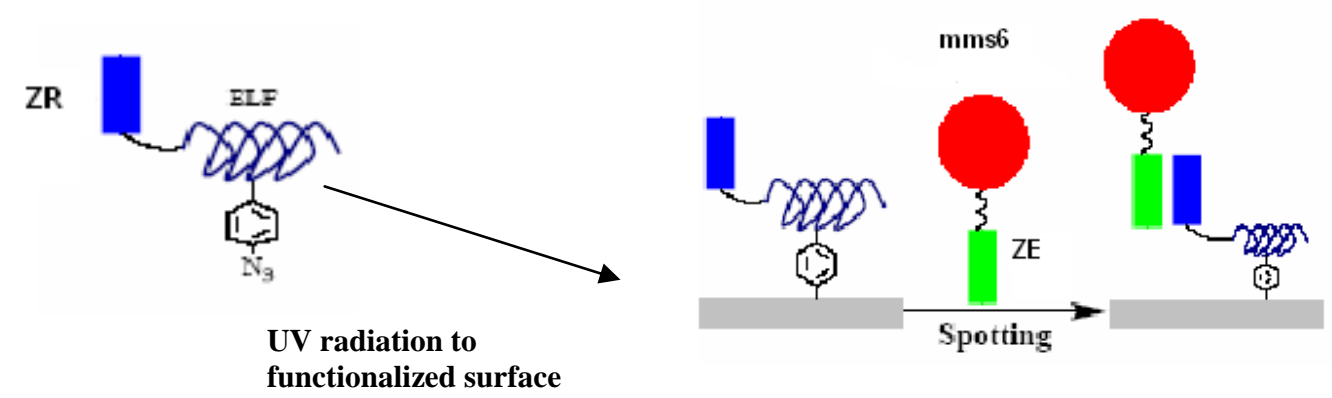

Figure 9.3. Proposed artificial polypeptide design for the Mms6-ZE immobilization

We have investigated several parallel approaches to achieve this goal, as outlined below.

Method 1. Construct ZE- Mms6 plasmid system for the expression of ZE-Mms6 fusion protein (work carried out in Dr.Tirrell's lab at Caltech).

First, the ZE segment in PQE60_ (ZEHis-ZRELF) plasmid vector [9], was PCR copied with the BamH1 cutting sites. The Mms6 plasmid (pTrc His-mms6) [8] was cut open at the BamH1 site (right before the Mms6 gene). Later, the ZE insert was ligated into the Mms6 plasmid, and was transformed into XL1-blue cells. Colonies were grown and the transformed plasmid DNA's were purified using mini-prep. The correct colonies were identified by DNA sequencing. Parallely, the noncanonical aminoacids protein expression of ZR-ELF was performed from the procedures developed by Zhang et al [9] .

Future work on these biopolypeptide scaffold design will be performed in our collaborator Dr. Marit Nilsen-Hamilton's laboratory, where the development of different Mms6-ZE fusion gene constructs (Method 2 and Method 3) as well as the protein expressions of ZE-Mms6 and ZR-ELF will be performed similar to the procedures followed for both natural and noncanonical aminoacid protein expression [9]. 


\section{Method 2. Construct ZE- Mms6 (C-terminal fragments) plasmid system for the expression of ZE-Mms6 (C-terminal fragments) fusion protein.}

First, the PQE60_(ZEHis-ZRELF) plasmid vector, was incorporated with spe1 site

after the ZE gene fragment using site-directed mutagenesis. The mms6 c-terminal fragments (25, 40 aminoacids) was PCR copied with spe 1 cutting sites. Later they will be ligated into PQE60_(ZEHis-ZRELF) plasmid vector at the spe1 site. Upon successful gene sequencing, protein expression will be performed similar to the natural aminoacids protein expression.

\section{Method 3. Direct fusion of Mms6 with ELF gene.}

The ELF portion was PCR copied from PQE60_(ZEHis-ZRELF) plasmid vector, with the additional BamH1 cutting sites. The Mms6 plasmid was cut open at the BamH1 site (right before the Mms6 gene, and the ELF segment was fused into the Mms6 vector). The ligation will be performed and colonies will be checked for successful gene sequence. Upon successful gene sequencing, protein expression will be performed similar to the noncanonical aminoacid protein expression protocol [9].

Upon successful polypeptide scaffold design, synthesis of magnetic crystals with controlled architecture and size with novel properties could be realized.

To conclude, this thesis work has contributed and emphasized the role of polymer templates the novel bottom-up approach to nanocomposite materials design. This method will open up new paths for facile synthesis of complex materials. Further, the tailoring of these polymers into different functionalities has paved way for biomedical applications such as in cross-linking polymer gels for cartilage repair and cationic and glucose responsive polymer gels as gene and drug delivery materials. 


\subsection{References}

1. Michael D. Determan, L.G., Chieh-Tsung Lo, P. Thiyagarajand and Surya K. Mallapragadaa The $\mathrm{pH}$ and temperature dependent phase behavior of a PEO-PPO$P E O$ based pentablock copolymer in aqueous media Physical Review E, 2008. In press.

2. Nowak Andrew, P., et al., Rapidly recovering hydrogel scaffolds from selfassembling diblock copolypeptide amphiphiles. Nature FIELD Full Journal Title:Nature, 2002. 417(6887): p. 424-8.

3. Deming, T.J., Polypeptide and polypeptide hybrid copolymer synthesis via NCA polymerization. Advances in Polymer Science, 2006. 202(Peptide Hybrid Polymers): p. 1-18.

4. Bellomo, E.G., et al., Stimuli-responsive polypeptide vesicles by conformationspecific assembly. Nature Materials, 2004. 3(4): p. 244-248.

5. Deming, T.J., Polypeptide hydrogels via a unique assembly mechanism. Soft Matter, 2005. 1(1): p. 28-35.

6. Deming, T.J., Synthetic polypeptides for biomedical applications. Progress in Polymer Science, 2007. 32(8-9): p. 858-875.

7. Breedveld, V., et al., Rheology of Block Copolypeptide Solutions: Hydrogels with Tunable Properties. Macromolecules, 2004. 37(10): p. 3943-3953.

8. Prozorov, T., et al., Protein-mediated synthesis of uniform superparamagnetic magnetite nanocrystals. Advanced Functional Materials, 2007. 17(6): p. 951-957.

9. Zhang, K., M.R. Diehl, and D.A. Tirrell, Artificial Polypeptide Scaffold for Protein Immobilization. Journal of the American Chemical Society, 2005. 127(29): p. 1013610137.

10. Son, S., C. Tanrikulu, and D.A. Tirrell, Stabilization of bzip peptides through incorporation of fluorinated aliphatic residues. ChemBioChem, 2006. 7(8): p. 12511257.

11. Shen, W., et al., Assembly of an Artificial Protein Hydrogel through Leucine Zipper Aggregation and Disulfide Bond Formation. Macromolecules, 2005. 38(9): p. 39093916. 


\subsection{Acknowledgements}

I would like to thank my advisor Dr. Surya Mallapragada for her guidance and constant support throughout the PhD research. Thanks to Dr. Mufit Akinc, Dr. Klaus Schmidt-Rohr and Dr. Alex Travesset for their suggestions and fruitful discussions on the biomineralization project. Thanks to my Program of Study Committee for their gracious support throughout this thesis work. Thanks to my biomineralization collaborative project group members Yusuf Yusufoglu, Drew Enlow, Yanyan Hu, Joshua Anderson and Aditya Rawal. Also I would like to thank Dr. David Tirrell and Dr.Brad Olsen at CalTech, Dr. Marit Nilsen-Hamilton at ISU, Dr. Tim Deming and Jennifer Yang at UCLA for their help with teaching techniques related to artificial polypeptide scaffold synthesis methods. Thanks to Dr. P. Thiyagarajan, Kevin Lo, Denis Wozniak and Sonke Seifert at Argonne National Laboratory for their extensive help and technical support with the small angle scattering experiments. Thanks to my fellow colleagues and group members whose help in numerous ways along the project: Sergiy Peleshanko, Ankit Agarwal, Sim-Siong Wong, Michael Determan, Tanya Prozorov and Bingqi Zhang. Also I would like to thank Dr. Zhiqun Lin and Mathew Goodman for their help with the hydrogel project. Like to thank several undergraduates who aided in the experimental work of this thesis projects: James Cox, Colin Paul and Juvan Veloz.

I am grateful to my parents, for their love, support and encouragement throughout my career, and to my brother and sister for their support and love.

This work was performed at Ames Laboratory under Contract No. DE-AC02-

07CH11358 with the U.S. Department of Energy. The United States government has assigned the DOE Report number IS-T 2764 to this thesis. 